\title{
Ultrasonic Methods to Measure Shear Wave Velocity and Absorption for Tissue Viscoelasticity \\ Characterization
}

by

Zhen Qu, M.A.Sc.

\author{
A Thesis submitted to \\ the Faculty of Graduate and Postdoctoral Affairs \\ in partial fulfillment of the requirements for the degree of \\ Doctor of Philosophy \\ in Electrical and Computer Engineering
}

Ottawa-Carleton Institute for Electrical and Computer Engineering

Department of Systems and Computer Engineering

Carleton University

Ottawa, Ontario

April, 2017

Copyright (C) 2017 - Zhen Qu 


\section{Abstract}

Characterization of the viscoelasticity for soft tissues is useful for medical diagnosis. Various ultrasound and magnetic resonance-based techniques have been developed over the past few decades. With ultrasound methods, generating and tracing a shear wave (SW) propagating within the soft tissue was one of the promising approaches to measure the SW velocity and absorption, which were the key parameters to derive the tissue viscoelasticity. When the SW method was employed, several problems, such as undesired SW reflection, motion artifact and SW diffraction could occur in practical experimental configuration, which caused on SW measurements. Advanced ultrasound imaging system with high frame rate may be able to overcome these problems, yet the configuration of such system was complex and thus expensive. In this thesis, methods to solve the abovementioned problems were proposed by using conventional ultrasound imaging system with a relatively less complex design and low frame rate. In the proposed methods, the undesired SW reflections were able to be distinguished and removed from the observed SW in the spatial domain using the scanning time delay in the B-mode measurement of conventional ultrasound system. The causes of the motion artifact were examined through the temporal domain analysis, and temporal frequency filter was used to reduce the undesired motions. In addition, the measurement configuration to minimize the diffraction effect on the SW measurement was investigated. Furthermore, a numerical method to compensate the diffraction effect was developed for the absorption measurement. The effectiveness of these proposed methods was verified by numerical 
simulations as well as by experiments using soft-tissue mimicking phantom specimens. The proposed methods were finally applied for in-vivo measurements and successfully demonstrate the stiffness difference of an upper arm muscle with and without muscle contraction. 


\section{Acknowledgments}

There are many people I would like to thank who helped me throughout these years.

First of all, I would like to thank my supervisor Prof. Yuu Ono for his invaluable support and advice during my time at Carleton University. This thesis work would not be accomplished without his guidance and encouragement. I would also like to thank Prof. Andy Adler for his generosity to allow me to use the ultrasound imaging system for my thesis research, and Prof. Adrian D. C. Chan and Dr. Andrew Marble for their valuable academic advice. Many thanks to my thesis committee members, Prof. Hakim Bendada, Prof. Hilmi Dajani, Prof. Jeremy Laliberte, Prof. Eran Ukwatta for their constructive comments, and Prof. Michel Gaulin for his great organization of the thesis defense.

I would like to acknowledge the Natural Sciences and Engineering Research Council of Canada (NSERC) for their financial support of the research presented in this thesis, and the Carleton University for the graduate scholarship.

Thanks to my colleagues Andy Huang, Ibrahim AlMohimeed, Yuqing Qiu, Dr.

Bruno Trindade, Dr. Shermeen Nizami, and Dr. Ce Peng for their assistance and advice. They have always generously shared their valuable knowledge and experience with me.

Finally, I would like to give my special thanks to my parents, my grandparents, my wife and my son Carson for always supporting and encouraging me all the time! 


\section{Table of Contents}

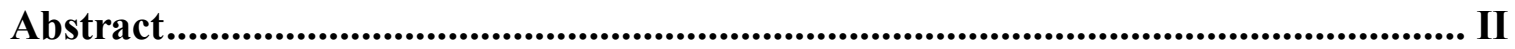

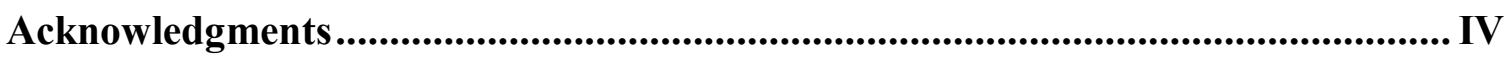

Table of Contents.................................................................................................................

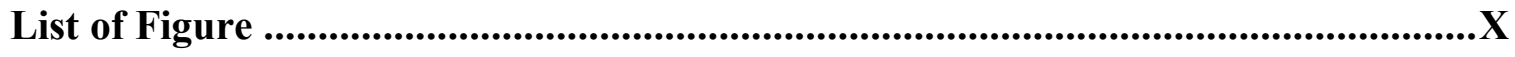

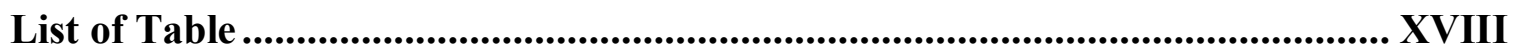

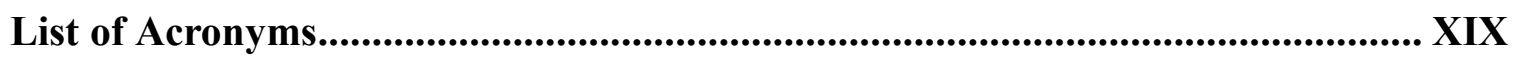

List of Symbols ................................................................................................................ XXI

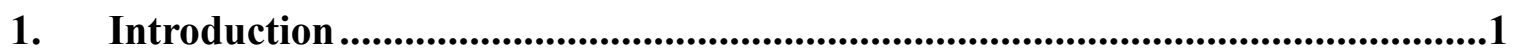

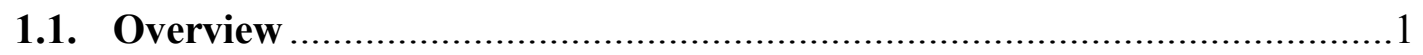

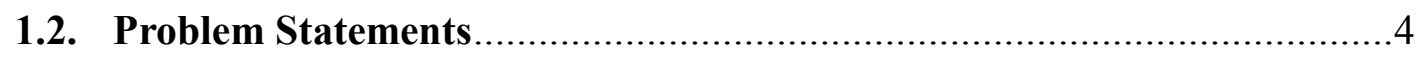

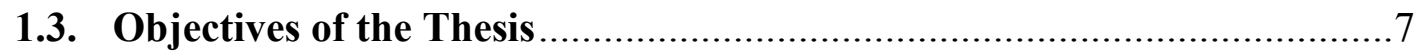

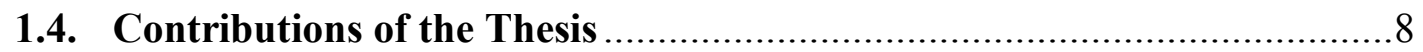

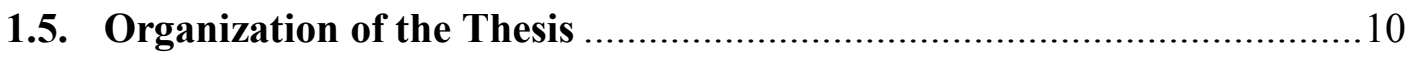

2. Background and Technical Review .....................................................................13

2.1. Mathematical Basis of Tissue Characterization ......................................13

2.2. Notable Techniques of Viscoelasticity Measurement ................................18 
2.3. Clinical Applications with Ultrasound Technique

3. Development of Shear Wave Velocity Measurement Method Using Focused Ultrasound and External Shear Wave Source ..........................................................31

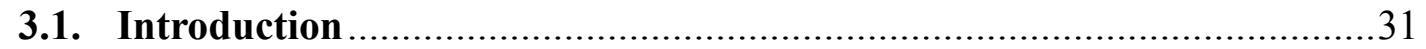

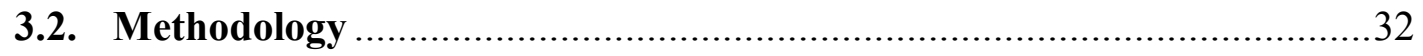

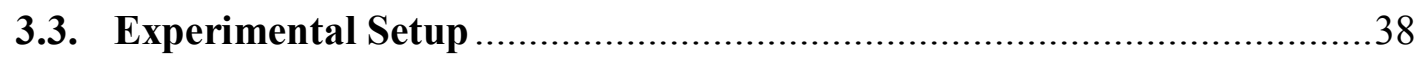

3.3.1. Hardware Equipments and DAQ Program.................................38

3.3.2. Fabrication of Tissue-mimicking Phantom ................................4 42

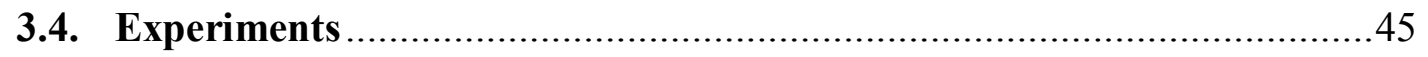

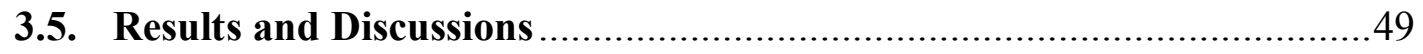

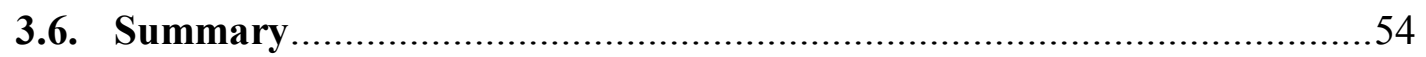

4. Modification of the Shear Wave Velocity Measurement Method.......................55

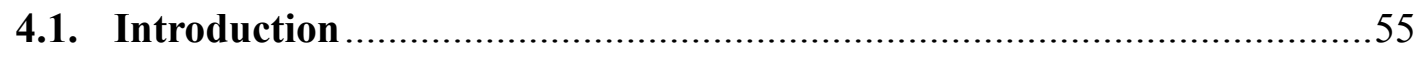

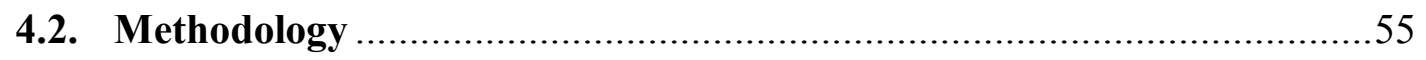

4.2.1. Modification of Measurement Model ........................................55

4.2.2. Removal of Shear Wave Reflection ..............................................58

4.2.3. Selection of Shear Wave Frequency .......................................61

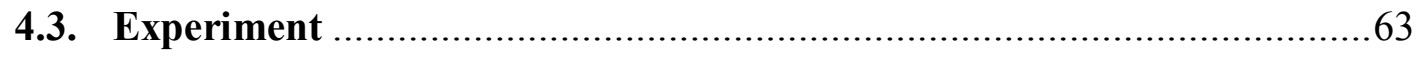

4.3.1. Experimental Configuration .............................................6 63

4.3.2. Experimental Result ....................................................65 
4.4. Discussions

4.4.1. Numerical Validation of Phantom Experiment

4.4.2. Influence of Shear Wave Reflection on Shear Wave Velocity

Measurement.

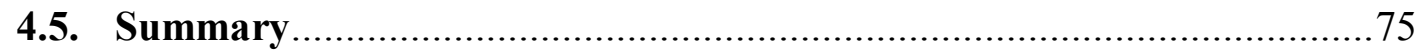

5. A Method to Reduce Motion Artifacts.................................................................76

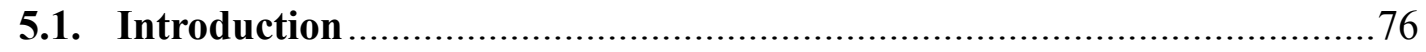

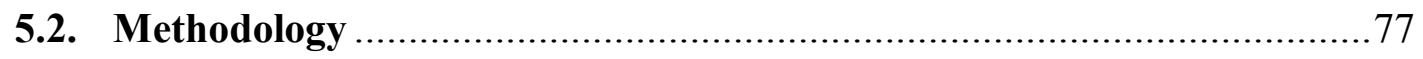

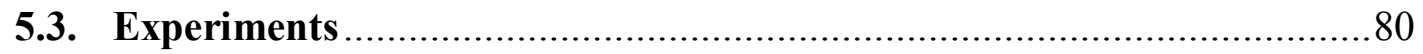

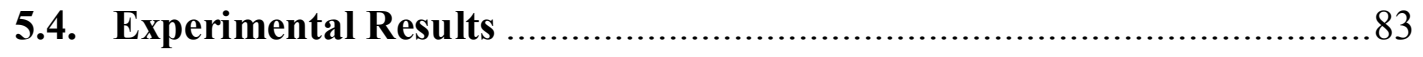

5.4.1. Motion Artifact Due to Experimental Environment ......................83

5.4.2. Motion Artifact Due to Handheld Probe ...................................... 84

5.4.3. Shear Wave Velocity Measurement ........................................85

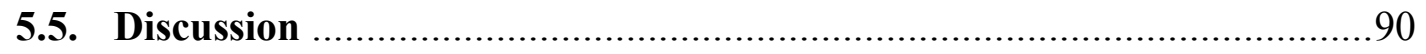

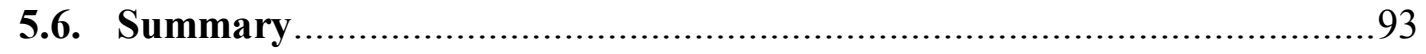

6. In-vivo Measurement of Shear Wave Velocity ..............................................95

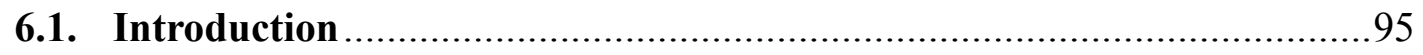

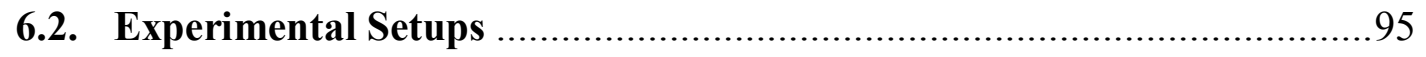

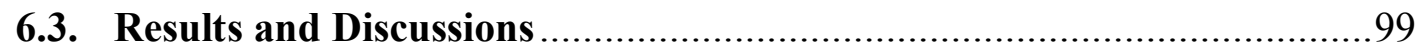

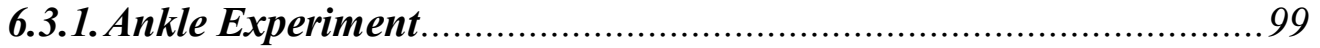


6.4. Summary. 106

7. Measurement of Shear Wave Absorption with Correction of the Diffraction Effect .07

7.1. Introduction 107

7.2. Methodology 108

7.2.1. Measurement Model of Shear Wave Absorption 108

7.2.2. Numerical Simulation of Diffraction Effect. 113

7.3. Experiment 122

7.3.1. Experimental Setup 122

7.3.2. Measurement of Shear Wave Absorption

7.4. Results and Discussion 126

7.5. Summary

8. Conclusions

8.1. Summary of Thesis Research 132

8.2. Scope and Limitation

8.3. Future Research

Appendix A. Mode Conversion from Shear Wave to Longitudinal Wave

Appendix B. Measurement of Tissue Displacement Using Ultrasound RF Signal..139

Appendix C. Undersampling of Tissue Displacement in Temporal Domain .143 
Appendix D. Frequency-Domain Filtering ...............................................................145

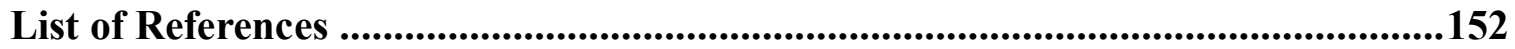




\section{List of Figure}

Figure 2.1. Moduli variance with different types of human tissues (reproduced from [41]).

Figure 2.2. Voigt material model. .15

Figure 2.3. Particle oscillation of shear wave. .16

Figure 2.4. Schematic to explain the principle of compressional elastography (adopted from [45]). .20

Figure 2.5. Experimental configuration of Yamakoshi's method (reproduced from [7]).21

Figure 2.6. Experimental configuration for measurement of crawling wave, (1) and (2)

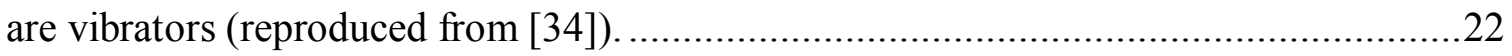

Figure 2.7. Schematic concept of acoustic radiation force to excite a transient SW (adopted from [45]). .24

Figure 2.8. Measurement configuration of SDUV (reproduced from [20]).....

Figure 2.9. Generation of the supersonic plane shear wave (adopted from[16]). .26

Figure 2.10. A schematic of the measurement configuration and observed SW patterns of CUSE (reproduced from [24]). 27

Figure 3.1. The procedure of B-mode scan using focused ultrasound. .32

Figure 3.2. Measurement model of SW velocity. .33 
Figure 3.3. Flow chart of signal processing procedure for acquired ultrasound rf signals.

Figure 3.4. Highest SW frequency with respect to SW velocity to satisfy the Nyquist sampling theorem with ultrasound scanning system used in this chapter. .38

Figure 3.5. Equipments used in the experiment.

Figure 3.6. Verification of pulse repetition frequency and B-mode scan frame rate......41

Figure 3.7. (a) Photo and (b) ultrasound B-mode image of a soft tissue mimicking phantom with combination of agar and gelatin phantoms used in the experiments.

Figure 3.8. Experimental configuration of SW velocity measurement for (a) the individual phantom, and (b) the combined phantom. .48

Figure 3.9. Observed SW displacements (a) in the entire FOV and (b) along $z$-direction at $x=0$, and (c) spatial frequency spectrum of the displacement shown in (b). Those of filtered SW displacements were presented in (d)-(f) respectively.

Figure 3.10. Measured SW velocity obtained from the filtered SW displacements of (a) the agar phantom and (b) the gelatin phantom .51

Figure 3.11. Configuration of the measurement on SW impulse using M-mode. .52

Figure 3.12. Measured SW velocity of the combined phantom. .53

Figure 4.1. Modified measurement model of SW velocity. .57

Figure 4.2. Normalized spatial frequencies of forward and backward SWs with respect to the SW velocity when $v_{\text {scan }}=2.26 \mathrm{~m} / \mathrm{s}$. .59 
Figure 4.3. (a) The dispersion of normalized spatial frequencies of forward and backward SWs. (b) The coverages of normalized spatial frequencies of forward and backward SWs when $1.02 \mathrm{~m} / \mathrm{s} \leq v_{S} \leq 10 \mathrm{~m} / \mathrm{s} . v_{\text {scan }}=2.26 \mathrm{~m} / \mathrm{s}$.

Figure 4.4. The highest and lowest applicable SW frequency with respect to shear wave velocity.

Figure 4.5. A structure (top left), photograph (top right), and B-mode ultrasound image (bottom) of the phantom fabricated. .64

Figure 4.6. Experimental configuration. .65

Figure 4.7. Measurement results of (a) filtered backward $\mathrm{SW}\left(V_{B}\right)$ in the phantom, (b) profile of SW waveform on the centerline as indicated in (a), and (c) the normalized spatial frequency spectrum of filtered backward $\mathrm{SW}\left(V_{B}\right)$ at centerline. .66

Figure 4.8. (a) Measurement result of SW velocity, dash circle indicated the area of the inclusion used to determine the mean value of the SW velocity. (b) A superposition of Bmode image and SW velocity. .68

Figure 4.9. Simulation results of (a) filtered backward $\mathrm{SW}\left(V_{B}\right)$, (b) profile of $\mathrm{SW}$ waveform on the centerline as indicated in (a), and (c) the normalized spatial frequency spectrum of filtered backward $\mathrm{SW}\left(V_{B}\right)$ at centerline. .70

Figure 4.10. (a) SW velocity that calculated from the simulation, and (b) the difference between the calcualtion result and actual value of the SW velocity. Dash-circle indicated the area of the inclusion used to determine the mean value of the SW velocity. .71

Figure 4.11. Estimation error of SW velocity without using the spatial frequency filtering. Error bar indicated the $+/-$ standard deviation .74 
Figure 4.12. Estimation error of SW velocity with using the spatial frequency filtering. Star-dash-line represented the simulation with actual $v_{S}=2 \mathrm{~m} / \mathrm{s}$, while square-dash-line represented $4 \mathrm{~m} / \mathrm{s}$, triangle-dash-line represented $6 \mathrm{~m} / \mathrm{s}$, and circle-dash-line represented 8 $\mathrm{m} / \mathrm{s}$. .75

Figure 5.1. Flowchart of signal processing procedure to reduce the motion artifact. ......79

Figure 5.2. Experimental configuration for motion artifact reduction: (a) test without SW in a stage-holding probe design, (b) test without SW in a hand-holding probe design, and (c) SW velocity measurement in a hand-holding probe design.

Figure 5.3. Temporal frequency spectrum of the first measurement procedure in which SW was not applied, and ultrasound probe was held by the positioning device.

Figure 5.4. Temporal frequency spectrum of the second measurement (SW was not applied, ultrasound probe was hand held). .84

Figure 5.5. Temporal frequency spectrum of the second measurement (SW was applied, ultrasound probe was hand held)..... .85

Figure 5.6. Observed SW displacements (a) in the entire FOV and (b) along $z$-direction at centerline $(x=0)$, and (c) magnitude spectrum of (b). And filtered SW displacements (d) in the entire FOV and (e) along z-direction at centerline, and (f) magnitude spectrum of (e). Results above were obtained with the reduction of motion artifact. .87

Figure 5.7. Observed SW displacements (a) in the entire FOV and (b) along $z$-direction at centerline, and (c) magnitude spectrum of (b). And filtered SW displacements (d) in the entire FOV and (e) along z-direction at centerline, and (f) magnitude spectrum of (e). Results above were obtained without the reduction of motion artifact. .88 
Figure 5.8. Results of SW velocity (a) with motion artifact reduction process, and (b) without motion artifact reduction process

Figure 5.9. Calculated shear velocities versus to their actual values at different SNRs (a) without, and (b) with the process of motion artifact reduction, while black-dot-line represented the simulation with $10 \mathrm{~dB}$ WGN, Blue-dot-line represented $20 \mathrm{~dB}$, and reddot-line represented $30 \mathrm{~dB}$. .91

Figure 5.10. The change of the normalized spatial frequency of backward wave $\left(V_{B}\right)$ with respect to the SW velocity. .93

Figure 6.1. A schematic (top) and photograph (bottom) of the experimental configuration of shear wave velocity measurement at ankle.

Figure 6.2. Experimental configuration of shear wave velocity measurements at upper arm. .98

Figure 6.3. Temporal frequency spectrum averaged in the entire field-of-view. The dashline represented the spectrum before the motion artifact reduction and the solid-line represented the spectrum after the motion artifact reduction.

Figure 6.4. Results of ankle experiment: (a) B-mode image, (b) SW velocity image, and (c) a superposition of B-mode image and SW velocity image. Arrows indicated the Achilles tendon. 101

Figure 6.5. The change of the mean value of the SW velocity with respect to the temporal frames within the ROIs as indicated by the dash-box in Fig. 6.4(b). The result in the ROI of tendon was plotted in (a) and that of muscle was plotted in (b). 102 
Figure 6.6. Results of arm measurement: (a) B-mode image, (b) observed SW displacement, (c) filtered SW displacement, and (d) SW velocity image. Dash-boxs in (d) were the ROIs to indicate the bicep muscle. Those results at frame \#10 (0.3 seconds after the start of the measurement), \#50 (1.7 seconds), \#90 (3.0 seconds), \#130 (4.3 seconds), and \#170 (5.7 seconds) were illustrated from top to bottom.

Figure 6.7. The change of the mean value of the SW velocity within the ROI in Fig. 6.6(d) with respect to the temporal frames. .105

Figure 7.1. Measurement model of SW absorption. 110

Figure 7.2. An example of measured SW amplitude variations: (a) SW amplitude map over entire FOV, (b) amplitude profile along the centerline, and (c) amplitude profile at $z$ $=12 \mathrm{~mm}$.

Figure 7.3. The geometry of Fresnel diffraction of a rectangular source with considering the interference between induced and reflected waves. .114

Figure 7.4. The appearance of the SW displacements (left) and amplitudes (right) calculated from different size of the SW sources. The SW wavelength $\left(\lambda_{S}\right)$ was $10 \mathrm{~mm}$, and the characteristic sizes $(a)$ of the SW source were $0.5 \mathrm{~mm}, 2.5 \mathrm{~mm}, 5 \mathrm{~mm}, 10 \mathrm{~mm}, 20$ $\mathrm{mm}$, and $40 \mathrm{~mm}$, from top to bottom. 115

Figure 7.5. A simulation of amplitude change due to diffraction effect on centerline with respect to a normalized distance. The $k_{S} a$ value was 10 . 116

Figure 7.6. Simulations of amplitude change due to diffraction effect on centerline with respect to a normalized distance. Black-line represented $k_{S} a=2$, blue-line represented $k_{S} a$ $=5$, green-line represented $k_{S} a=10$, and red-line represented $k_{S} a=20$. 
Figure 7.7. Simulation of SW amplitude change due to diffraction with $k_{S} a=10$, the actual $S$-range was $[1,6]$ (top), and was mistakenly recorded to $S=[0.5,5.5]$ (middle), and $S=[1.5,6.5]$ (bottom).

Figure 7.8. Curve fitting results (indicated by dot-lines) of the SW amplitude with positioning error (indicated by solid-lines) in Fig. 7.7.

Figure 7.9. Calculation error on the amplitude change of diffraction effect due to positioning error. Red-circle dash line represented the positioning error for $S+0.5$, and blue-circle dash-line represented the positioning error for $S-0.5$.

Figure 7.10. SW velocity image of the gelatin phantom.

Figure 7.11. A simulation of amplitude change due to diffraction effect on centerline with respect to the length $z$. The $k_{S} a$ value was 3.32 .

Figure 7.12. Estimation error of SW absorption due to the positioning error of $z$-location for $k_{S} a=3.32$. Red-circle dash-line represented the positioning error of $z+1 \mathrm{~mm}$, and blue-circle dash-line represented the positioning error of $z-1 \mathrm{~mm}$. 123

Figure 7.13. Experimental configuration of SW absorption measurement. 126

Figure 7.14. The experimental result of (a) induced $\mathrm{SW}$ displacement $\left(V_{I}\right)$, (b) $\mathrm{SW}$ amplitude $\left(A_{I}\right)$, and (c) the SW amplitude on the centerline $(x=0)$ in (b).

Figure 7.15. Comparison between the simulation result (a) and experimental result (b) of the SW amplitude. .128

Figure A.1. Induced and reflected SWs with mode conversion. 138

Figure A.2. Energy reflection coefficient of SW. 138 
Figure C.1. A demonstration of SW displacement in (a) both temporal and spatial domain, (b) only temporal domain at a certain $(x, z)$, and (c) only spatial domain at a certain $(x, t)$.

Figure D.1. The spatial SW displacement (a) before the spatial filtering, and (b) after the spatial filtering. The frequency spectrum at centerline $(x=0)$ of each displacement was averaged over 180 frames and plotted in (c). .146

Figure D.2. The spatial SW displacement (a) before the spatial filtering, and (b) after the alternative spatial bandpass filtering. The frequency spectrum at centerline $(x=0)$ of each displacement was averaged over 180 frames and plotted in (c). 148

Figure D.3. SW velocity calculated with the alternative spatial filtering.

Figure D.4. A superposition of B-mode image and SW velocity image. Arrows indicated the Achilles tendon. 150

Figure D.5. The change of the mean value of the SW velocity with respect to the temporal frames within the ROIs as indicated by the dash-box in Fig. D.3. The result in the ROI of tendon was plotted in (a) and that of muscle was plotted in (b). Both results were calculated by using the alternative $\boldsymbol{B P F}_{\text {spatial }}$. 151 


\section{List of Table}

Table 3.1. A comparison of SW velocity obtained from different methods.

Table 4.1. Comparison between experimental and simulation results.

Table 7.1. Five regions in simulations to investigate the error due to positioning error.118

Table 7.2. Twenty one regions in phantom experiment to investigate the error due to positioning error.

Table 7.3. A comparison between our result and published results using similar phantom specimens.

Table D.1. Shear velocities calculated using original $\boldsymbol{B P F}_{\text {spatial }}$ and alternative $\boldsymbol{B P F}_{\text {spatial }}$. 


\section{List of Acronyms}

\begin{tabular}{|c|c|}
\hline Acronyms (Sort by Alphabet) & Definition \\
\hline $\mathrm{ARF}$ & Acoustic radiation force \\
\hline ARFI & Acoustic radiation force imaging \\
\hline $\mathrm{BPF}$ & Bandpass filter \\
\hline$B P F_{\text {spatial }}$ & Spatial bandpass filter \\
\hline$B P F_{\text {temporal }}$ & Temporal bandpass filter \\
\hline CUSE & Comb-push ultrasound shear elastography \\
\hline DAQ & Data acquisition \\
\hline $\mathrm{DC}$ & Direct current \\
\hline FOV & Field of view \\
\hline $\mathrm{FOV}_{\mathrm{x}}$ & Field of view in depth \\
\hline $\mathrm{FOV}_{\mathrm{z}}$ & Field of view in length \\
\hline LPF & Low pass filter \\
\hline LW & Longitudinal wave \\
\hline MRI & Magnetic resonance imaging \\
\hline PRF & Pulse repetition frequency \\
\hline $\mathrm{rf}$ & Radio frequency \\
\hline ROI & Region of interest \\
\hline SDUV & Shear wave dispersion ultrasound vibrometry \\
\hline SMURF & Spatially modulated ultrasound radiation force \\
\hline
\end{tabular}




\begin{tabular}{l|l}
\hline SNR & Signal-to-noise ratio \\
\hline SSI & Supersonic shear imaging \\
\hline STD & Standard deviation \\
\hline SW & Shear wave \\
\hline SWEI & Shear wave elasticity imaging \\
\hline WINDOW & Window function \\
\hline WGN & White Gaussian noise \\
\hline 2D & Two dimensional \\
\hline
\end{tabular}




\section{List of Symbols}

\begin{tabular}{|c|c|}
\hline Symbols (Sort by Alphabet) & Definition \\
\hline$a$ & $\begin{array}{l}\text { Characteristic size of rectangular SW source in } x \text { - } \\
\text { direction (depth) }\end{array}$ \\
\hline$A_{B}$ & Amplitude of backward SW \\
\hline$A_{F}$ & Amplitude of forward SW \\
\hline$A_{I}$ & Amplitude of induced SW \\
\hline$A_{r f}$ & Amplitude of the received rf signal \\
\hline$A_{S}$ & Initial SW amplitude at the SW source \\
\hline$A T T$ & Attenuation due to the diffraction effect \\
\hline$b$ & $\begin{array}{l}\text { Characteristic size of rectangular SW source in } y \text { - } \\
\text { direction (width) }\end{array}$ \\
\hline$c_{0}$ & Ultrasound speed \\
\hline$E$ & Young's modulus \\
\hline$f_{F R}$ & Frame rate of imaging system \\
\hline$f_{M A}$ & Frequency of motion artifact \\
\hline$f_{\text {measured }}$ & Aliased SW frequency \\
\hline$f_{S}$ & SW frequency \\
\hline$G$ & Shear elasticity (shear modulus) \\
\hline $\mathcal{H}_{z}\{\}$ & Hilbert transform \\
\hline$I$ & In-phase signal \\
\hline $\operatorname{Im}\{\}$ & Imaginary component of a complex signal \\
\hline
\end{tabular}




\begin{tabular}{|c|c|}
\hline$j$ & Imaginary unit \\
\hline$K$ & Bulk modulus \\
\hline$k_{S} a$ & $\begin{array}{l}k_{S} a \text { value, a product of SW wavelength and the } \\
\text { characteristic size of SW source }\end{array}$ \\
\hline$k_{S}$ & SW wavenumber \\
\hline$k_{B}$ & Wavenumber of backward SW \\
\hline$k_{F}$ & Wavenumber of forward SW \\
\hline$n$ & Integer multiple of $f_{F R}$ \\
\hline$N_{F}$ & Fresnel distance \\
\hline$Q$ & Quadrature signal \\
\hline$r_{I}$ & $\begin{array}{l}\text { Distance from an arbitrary point on SW source to } \\
\text { an arbitrary point on SW reflector }\end{array}$ \\
\hline $\boldsymbol{R e}\{\}$ & Real component of a complex signal \\
\hline$S$ & Normalized distance in length \\
\hline$S_{r e f}$ & Complex reference signal \\
\hline$S_{r f}$ & Received rf signal \\
\hline$S_{T}$ & Transmitted ultrasound signal \\
\hline$t$ & Time \\
\hline$\Delta t$ & Temporal sampling interval \\
\hline$u_{i}$ & Scatterer displacement along direction $i(x, y$, or $z)$ \\
\hline$v$ & Velocity of scatterer displacement \\
\hline$v_{L}$ & LW velocity \\
\hline$v_{\text {scan }}$ & B-mode scanning speed \\
\hline$v_{S}$ & SW velocity \\
\hline$\Delta v_{S_{-} H i g h}$ & $v_{S}$ change at higher $v_{S}$ value \\
\hline
\end{tabular}




\begin{tabular}{|c|c|}
\hline$\Delta v_{S-L o w}$ & $v_{S}$ change at lower $v_{S}$ value \\
\hline$V$ & SW displacement \\
\hline$V_{B}$ & Backward SW displacement \\
\hline$V_{F}$ & Forward SW displacement \\
\hline$V_{I}$ & Induced SW \\
\hline$V_{M A}$ & Displacement of undesired motion \\
\hline $\mathrm{V} \%$ & Volume percent \\
\hline W\% & Weight percent \\
\hline$x$ & Depth \\
\hline$y$ & Width/thickness \\
\hline Y & In-phase quadrature signal \\
\hline$z$ & Length \\
\hline$\Delta z$ & Spatial sampling interval \\
\hline$\alpha_{S}$ & SW absorption \\
\hline$\beta$ & Conversion index from displacement to phase \\
\hline$\Gamma_{S S}$ & $\begin{array}{l}\text { Amplitude of reflected SW from incident SW in } \\
\text { mode conversion }\end{array}$ \\
\hline$\varepsilon$ & Strain \\
\hline$\eta$ & Shear Viscosity \\
\hline$\theta$ & $\begin{array}{l}\text { Angle between the direction of ultrasound } \\
\text { transmission and scatterer displacement }\end{array}$ \\
\hline$\theta_{L}$ & Reflection angle of LW \\
\hline$\theta_{S}$ & Incident angle of SW \\
\hline$\lambda_{S}$ & SW wavelength \\
\hline$\mu$ & Shear viscoelasticity \\
\hline
\end{tabular}




\begin{tabular}{l|l}
\hline$\xi_{B}$ & Normalized spatial frequency of backward SW \\
\hline$\xi_{F}$ & Normalized spatial frequency of forward SW \\
\hline$\Delta_{F}$ & Spatial frequency change \\
\hline$\rho$ & Density \\
\hline$\sigma$ & Stress \\
\hline$\varphi_{B}$ & Angle between $r_{I}$ and $z$ \\
\hline$\varphi_{F}$ & Initial phase of the backward SW \\
\hline$\varphi_{I}$ & Initial phase of the forward SW \\
\hline$\varphi_{r f}$ & Initial phase of the induced SW \\
\hline$\varphi_{V}$ & Phase component of the rf signal \\
\hline$\varphi_{S}$ & Change of the phase of the rf signal \\
\hline$\varphi_{0}$ & Phase of velocity of the scatterer displacement \\
\hline$\omega_{d}$ & Initial phase of the rf signal \\
\hline$\omega_{i}$ & Doppler shift frequency \\
\hline$\omega_{S}$ & $\begin{array}{l}\text { Instantaneous angular frequency of received rf } \\
\text { signal }\end{array}$ \\
\hline$\omega_{0}$ & SW angular frequency \\
\hline$\nabla^{2}$ & Ultrasound angular frequency \\
\hline & Laplace operator \\
\hline & \\
\hline
\end{tabular}




\section{Chapter 1}

\section{Introduction}

This chapter is the introduction of this thesis. Motivation and the overview of the research are presented, followed by potential problems and the proposed solutions.

\subsection{Overview}

Characterization of the viscoelasticity for soft tissues is useful for medical diagnosis but considered a challenging topic which has been widely studied for decades. Two shear properties: shear elasticity and viscosity, are often referred to represent the tissue viscoelastic properties. Shear elasticity represents the stiffness of the material. In medical diagnosis, the determination of elasticity is a popular topic since it can be used to evaluate the soft tissue health according to the stiffness difference between normal and abnormal tissues [1]. Viscosity represents the resistance to the deformation of the material by shear stress. Although in most tissues, shear elasticity may be a primary parameter to characterise the tissue mechanical property and viscosity was neglected, many studies still suggested that not only the elasticity but also the viscosity was useful and could be an interesting index of tissue health as well [2], [3].

One way to determine the tissue viscoelastic properties was based on the analysis

of stress-strain behavior of a soft tissue specimen, which was commonly called a quasistatic measurement [1], [4]. Under compressive forces, soft tissue with different 
elasticity will exhibit different deformation, i.e. the greater strain indicates the smaller stiffness and vice versa, and the viscosity can be estimated by measuring the relaxation time of the specimen from the compressive force [5]. Another way to determine the viscoelasticity was based on tracing and analyzing a shear wave (SW) propagation inside a specimen, which was commonly called a dynamic measurement or SW measurement [4]. With such type of measurement, the shear elasticity can be estimated by measuring the velocity of SW $\left(v_{S}\right)$ and the density of the specimen. In addition, the viscosity can be determined by measuring both the $v_{S}$ and $\mathrm{SW}$ absorption coefficient $\left(\alpha_{S}\right)$ at a known SW frequency $\left(f_{S}\right)$, or from analyzing the $v_{S}$ dispersion [6]. Greater $\alpha_{S}$ or stronger $v_{S}$ dispersion indicates a greater amount of viscosity, and vice versa.

In practice, it may be difficult to precisely measure an external stress during invivo experiments, thus the quasistatic measurement usually only provides a relative contrast between the stiffer and softer materials based on their strain difference under the same amount of external stress. Besides, it could also be difficult to make the tissues or organs located at deeper area be appropriately compressed with the quasistatic measurement. As a contrast, SW measurement can measure the absolute value of the tissue viscoelasticity at any depth in the tissue of interest. Therefore, the SW measurement may be more competent and outperforms the quasistatic measurement.

Ultrasound and magnetic resonance imaging-based $\mathrm{SW}$ measurement methods have been developing rapidly through the past three decades [1], [4], [7]-[25]. Many clinical applications using either method have also been demonstrated [26]-[33].

In the ultrasonic method, the $v_{S}$ can be determined either through a time-of-flight technique by measuring the time spent on a certain distance which an impulse SW 
traveled through [10], or by analyzing the wavelength of a continuous SW waveform at a known $f_{S}$ [4]. The measurement of impulse SW required high frame rate ultrasound scanning system, while such requirement was not necessary for the measurement of continuous SW. The accurate measurement of $\alpha_{S}$, however, was usually more challenging than $v_{S}$. This was because in order to determine $\alpha_{S}$, the attenuation of the SW amplitude caused by the mode conversion, scattering effect and diffraction effect should be firstly compensated, and such compensation requires mathematical model and intense computation.

Currently, the most promising implementation of the SW measurement could be tracing an impulse SW by using a high frame rate plane wave ultrasound system. However, the design of such system could be rather complex with intense signal processing involved. For instance, an advanced SW measurement systems, such as supersonic shear imaging [16], may be expensive, and thus not be available for some research organizations. Although the SW measurement systems using continuous SW traced by the conventional B-mode scan of focused ultrasound were relatively less expensive, they experienced several practical experimental problems, such as the undesired SW reflections, motion artifacts, and diffraction effect on the measurement of SW.

Motivated by these problems and inspired by the innovations from the developed technologies, methods were proposed through this thesis research to address the experimental problems mentioned above. In this thesis, the SW measurement used a conventional B-mode scan of a focused ultrasound and a single external continuous SW source. Consequently, the ultrasound system was not only less expensive, but also more 
popular than the plane-wave-based ultrasound system. In addition, the use of a single external SW source can simplify the overall experimental configuration compared with the use of multiple SW sources, such as the crawling wave method [34], and/or reduce the complexity of the SW generation compared with the use of a radiation force technique [10]. With the proposed experimental configuration, both $v_{S}$ and $\alpha_{S}$ can be measured in the same experiment.

Detailed problem statements will be given in Section 1.2, and the thesis objectives will be presented in Section 1.3.

\subsection{Problem Statements}

Although the SW measurement using conventional B-mode scan and external SW source could be low in cost and simple in configuration, several practical problems exist. Errors may be added onto the measurement results of $v_{S}$ and/or $\alpha_{S}$, if these problems were not appropriately addressed. Three major problems addressed in this thesis are listed as follows:

\section{1) Undesired SW reflection from tissue boundary}

In some experiments, especially when the specimen of interest was relatively small, an induced SW may be reflected at the tissue boundaries, and thus both the induced and reflected SWs existed at the same time. As a result, the observed SW would be an interfered waveform if the continuous SW was employed. However, the conditions of the reflection, such as the location where the reflection occurred and the reflection coefficient, may be unknown. Therefore, the amplitude and 
phase of the reflected SWs were unpredictable. Unless the undesired reflected SW was removed from the observed SW waveform, the errors on the estimation of $v_{S}$ and $\alpha_{S}$ could occur.

Currently, an effective solution to remove the undesired SW reflection was a directional filtering technique with a plane wave ultrasound imaging system [35]-[37]. However, this technique was not applicable to the SW measurement with the conventional B-mode scanning system of the focused ultrasound used in this thesis research.

\section{2) Motion artifact on the measurement of shear wave displacement}

In an ideal SW measurement, any tissue displacement observed inside the specimen should be solely triggered by the SW propagation. However for a practical case, tissue displacement could also be generated by undesired motions. Motions from the equipment and/or environment in practical measurements, as well as involuntary in-vivo activities, such as heartbeat/pulse, breathing, and/or body movement [38], [39], may be unavoidable. All these undesired motions were considered as motion artifacts and would contribute offsets to the observed SW displacements. Consequently, this would result in errors on the estimation of $v_{S}$ and $\alpha_{S}$.

When the external SW source such as a vibrator or shaker was used, the measurement error due to the motion artifacts may become negligible with great magnitude of the induced SW by increasing the SW source power. However this could bring discomfort to the patient. Therefore, it was crucial to develop a 
method to reduce motion artifacts from the observed SW wave form with lower SW source power for practical clinical applications.

\section{3) Diffraction effect on the measurement of shear wave absorption}

The purpose of the $\alpha_{S}$ measurement was to determine the tissue viscosity. In principle, $\alpha_{S}$ can be estimated from the attenuation measurement of SW amplitude. However, the SW amplitude attenuated not only by the absorption, but also by the mode conversion, scattering and diffraction during the $\mathrm{SW}$ propagation in a specimen. Therefore, in order to estimate $\alpha_{S}$ from the measured SW attenuation, the mode conversion, scattering and diffraction should be taken into account. The diffraction was a nonlinear phenomenon [40], and the attenuation compensation was required based on the diffraction theory. In addition, the motion artifact could deform the SW waveforms, resulting in significant errors on the attenuation measurement. Because of such difficulties and challenges, measuring and analyzing the $v_{S}$ dispersion instead of directly measuring the SW attenuation to estimate the $\alpha_{S}$ was a common way, in order to determine the tissue viscosity. The $v_{S}$ dispersion could be obtained from the one measurement if the impulse SW having a broad frequency bandwidth was used. However, when the continuous SW was used, the dispersion method would require multiple measurements at different $f_{S}$, which may prolong the time of the measurement. Considering the discomfort of the patient, a quick measurement was desired by the one measurement of the SW attenuation to estimate the $\alpha_{S}$. Among the attenuation factors mentioned above, the mode conversion and scattering effects could be negligible with the proposed measurement configuration in this thesis. Thus, the 
compensation of the diffraction effect on the estimation of the $\alpha_{S}$ needed to be discussed.

\subsection{Objectives of the Thesis}

Estimating the $v_{S}$ and $\alpha_{S}$ parameters of soft tissues by using conventional B-mode scan of a focused ultrasound system is a meaningful approach. Its advantage is reflected not only through the reduced cost and configuration complexity, but also through the high popularity of the focused ultrasound system around the world. Therefore, a SW measurement using such ultrasound system with a relatively simple experimental configuration could both help reduce expenses, and show good potential in clinical applications. In order to realize the proposed measurement configuration as well as to address the problems stated in Section 1.2, the following objectives were expected to be achieved by the accomplishment of this thesis study.

1) Develop a measurement model of SW displacement by using B-mode scan of focused ultrasound and external shear wave source.

The measurement of both $v_{S}$ and $\alpha_{S}$ depended on a successful measurement of the SW displacement. A measurement model of the SW displacement was developed by using a conventional B-mode scan of a focused ultrasound system. An external SW source was used in this model to generate continuous SWs in a specimen at a chosen monotonic frequency. The Objectives $\mathbf{2}$ and $\mathbf{3}$ would be based upon this measurement model. 
2) Remove undesired SW reflections.

A method to remove the undesired reflected SW was proposed by using the Bmode scanning time delay of the conventional focused ultrasound system. The accomplishment of this objective would address Problem 1 in Section 1.2.

\section{3) Reduce motion artifacts.}

A method to reduce the motion artifacts from the observed SW waveform was proposed by using a temporal frequency filtering technique. The accomplishment of this objective would address Problem 2 in Section 1.2.

4) Minimize diffraction error on the estimation of SW absorption.

A measurement of $\alpha_{S}$ required a compensation of diffraction effect, while the diffraction effect was location-dependent. In order to minimize the error that occurs during the compensation of diffraction effect, the optimized measurement location was investigated. The accomplishment of this objective would address

\section{Problem 3 in Section 1.2.}

\subsection{Contributions of the Thesis}

Throughout this thesis research, several academic contributions were achieved upon the accomplishment of the thesis objectives. They were summarized and listed as follows.

1) A method to remove the undesired SW reflection was developed based on the use of B-mode scanning time delay of focused ultrasound. Removing undesired SW reflection made the estimation of $v_{S}$ and $\alpha_{S}$ more accurate. 
2) A method to reduce the motion artifacts was developed in the proposed ultrasound system which used low frame rate B-mode scanning and external SW source. Such method reduced the error on the measurement of SW displacement due to undesired tissue motions and made an in-vivo experiment feasible and reliable.

3) A method to obtain the $\alpha_{S}$ was developed based on the compensation of the diffraction effect on the SW attenuation measurement. Such method minimized error due to positioning error/misreading of the measurement location and made the measurement of $\alpha_{S}$ more accurate.

4) Several academic papers were published and submitted during the thesis research as listed below. These publications could be inspirations and/or references for future research.

\section{Publications:}

- $\quad$ Z. Qu and Y. Ono, "Measurement of shear wave velocity with a method to reduce motion artifact using conventional B-mode ultrasound scanning," to be submitted as journal paper. (Chapters 5-6)

- $\quad$ Z. Qu and Y. Ono, "Measurement of shear wave absorption with correction of the diffraction effect," to be submitted as journal paper. (Chapter 7)

- $\quad$ Z. Qu and Y. Ono, "Measurement of shear wave velocity for elastography using conventional B-mode scan of focused ultrasound," accepted by IEEE International Symposium on Medical Measurements and Application (MeMeA), May 7-10, 2017, Rochester, MN. (Chapter 4) 
- $\quad$ Z. Qu and Y. Ono, "A method to reduce the influence of reflected waves on shear velocity measurements using B-mode scanning time delay,” Japanese Journal of Applied Physics, Vol. 54, pp. 07HF01_1-6, 2015. (Chapter 3)

- $\quad$ Z. Qu and Y. Ono, "A method to reduce the influence of reflected shear waves on shear velocity measurement," 35th Symposium on Ultrasonic Electronics, pp. 547-548, December 3-5, 2014, Tokyo, Japan. (Chapter 3)

- $\quad$ Z. Qu and Y. Ono, "Ultrasonic measurements of shear wave propagation in soft tissue phantom for tissue characterization," IEEE International Symposium on Medical Measurements and Application (MeMeA), pp. 40-43, May 4-5, 2013, Gatineau, QC. (Chapter 7)

\subsection{Organization of the Thesis}

This thesis was constructed into eight chapters, briefly described as follows:

\section{Chapter 1. Introduction}

This chapter was the introduction of this thesis. Motivation and the overview of the research were presented, followed by potential problems and the proposed solutions.

\section{Chapter 2. Background and Technical Review}

This chapter was the literature and technical review. The mathematical basis of tissue characterization was firstly presented, and then the state-of-the-art measurement techniques were introduced. 
Chapter 3. Development of Shear Wave Velocity Measurement Method Using Focused Ultrasound and External Shear Wave Source

This chapter presented the basic concept of the SW velocity measurement method with a method proposed to remove the undesired SW reflections. Phantom experiments were demonstrated to verify the method.

\section{Chapter 4. Modification of Shear Wave Velocity Measurement Method}

This chapter improved the SW velocity measurement method to overcome a limitation of the method proposed in Chapter 3. The modified measurement method was verified by the experiments using a phantom with an inclusion structure. Performance of the measurement accuracy was also investigated through numerical calculation.

\section{Chapter 5. A Method to Reduce Motion Artifacts}

This chapter proposed a method to reduce the motion artifact based on the modified measurement method of SW velocity. The proposed method was verified by the phantom experiment and numerical calculation.

\section{Chapter 6. In-vivo Measurement of Shear Wave Velocity}

This chapter demonstrated the shear wave velocity measurements for in-vivo tissues with using the methods proposed in Chapters 4-5.

\section{Chapter 7. Measurement of Shear Wave Absorption with Correction of the Diffraction Effect}

This chapter discussed a measurement method of SW absorption. The measurement location was arranged at a certain place where the diffraction effect 
was relatively less sensitive to the change of the SW amplitude. Thus the calculation error due to diffraction would be minimized. The measurement method was verified by phantom experiment.

\section{Chapter 8. Conclusions}

The last chapter summarized and reviewed the thesis research and pointed out potential topics for future study. 


\section{Chapter 2}

\section{Background and Technical Review}

The purposes of this chapter are to introduce the mathematical basis of tissue characterization and to review the state-of-the-art ultrasound elastography and viscoelasticity measurement techniques along with their clinical applications.

\subsection{Mathematical Basis of Tissue Characterization}

It was well known that most of the soft tissues contain more than $70-\mathrm{w} \%$ of water, thus they can be considered as fluid-like solids, which means these kinds of materials have characteristics of both solids and fluids [41]. Therefore, a way to characterize such material was to analyze their viscoelastic properties, such as the elasticity and viscosity.

Elasticity is a physical property which describes the ability of a material to return to its original shape after stress is removed [42], and it mainly reflects the solid properties of material. There are three major moduli to represent the elasticity of a material: Young's modulus $(E)$, shear modulus $(G)$, and bulk modulus $(K) . E$ defines the ratio between tensile stress $(\sigma)$ and tensile strain $(\varepsilon)$ of a material as $E=\sigma / \varepsilon$ [43], while $G$ defines the ratio of shear stress and shear strain of a material. $K$ is defined as the ratio of the pressure and volume change. It should be noted that for a incompressible material (which applies to human soft tissues, which are nearly incompressible), it can be assumed that $E=3 G$ [14], [44], [45], besides, $E$ and $G$ have broader dynamic range among 
different types of human tissues than $K$ [41], The comparison between $K$ and $E$ (or $G$ ) is shown in Fig. 2.1. Thus $E$ and $G$ could be of more interest/significance for characterization of human tissues. On the other hand, viscosity $(\eta)$ describes the ability of material to resist deformation due to tensile stress or shear stress [42], and it mainly reflects the liquid properties of material. A Voigt material model, as shown in Fig. 2.2 [46], was used in this thesis to describe the viscoelastic materials. It is represented by a purely elastic spring connected in parallel to a purely viscous damper. Such model can be used to describe either a tensile viscoelastic material or a shear viscoelastic material. A material with only elasticity is called a purely elastic material, and a material with only viscosity is called a Newtonian fluid [47]. A soft tissue or soft tissue-like material is between these two extreme conditions and is called a viscoelastic material [48].

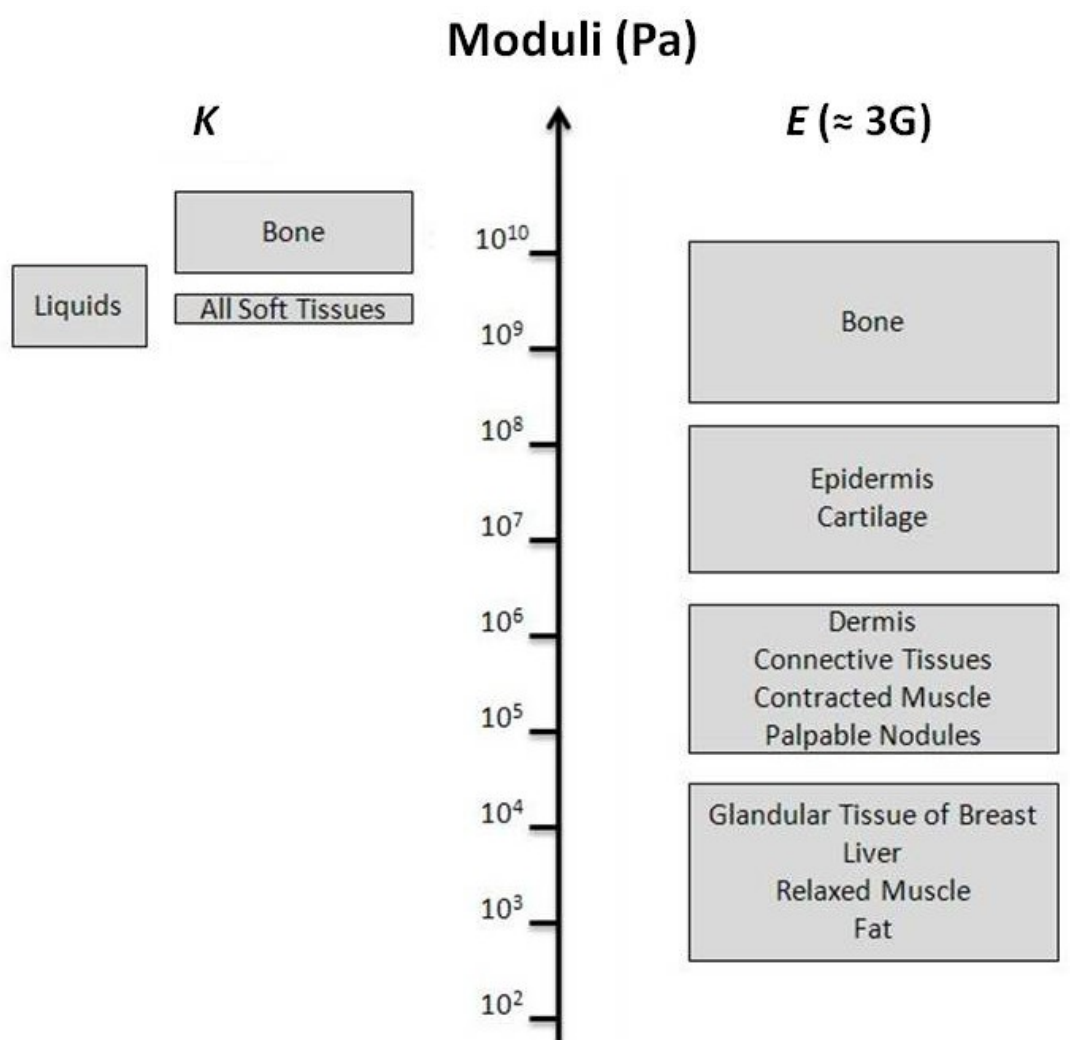

Figure 2.1. Moduli variance with different types of human tissues (reproduced from [41]). 


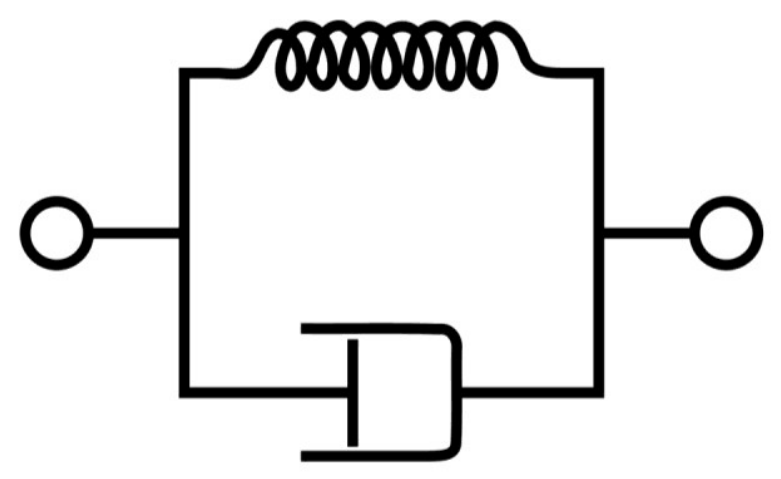

Figure 2.2. Voigt material model.

Basically, methods of determining the elasticity and/or viscosity were all based on the same objective, which was to find a way to detect the response of a tissue to a mechanical excitation. Then the mathematical relation between the response and excitation was used to derive the viscoelastic parameters [49]. The methods could be divided into two groups according to the temporal difference of the excitation [4], [14]: quasistatic measurement and dynamic measurement (also termed SW measurement). The quasistatic measurement method can determine $E$ by measuring the strain $\varepsilon$ caused by a known compressive force $\sigma$ and the area of such force, while the $\eta$ can be determined by measuring the relaxation time of the specimen from the compressive force with knowing of the $E[5]$. The SW measurement method can determine both $G$ and $\eta$ by tracing and analyzing a propagated SW in the specimen. In addition, although the quasistatic measurement provided contrast in terms of relative stiffness to detect abnormal lesions from healthy tissue, limitation also existed and restricted such technique [20]. For instance, it could be difficult to make compress organs located at deeper areas with the quasistatic measurement. Therefore, the SW measurement could be a more benefited method in such case. 
SW, also called transverse wave, is a moving mechanical wave that consists of particle oscillations occurring perpendicular to the direction of the energy transfer [44], as depicted in Fig. 2.3. In a viscoelastic material, the SW equation is given by [50]:

$$
\begin{aligned}
& \rho \frac{\partial^{2} u_{i}}{\partial t^{2}}-\mu \nabla^{2} u_{i}=0, \\
& \mu=G+j \omega_{S} \eta
\end{aligned}
$$

where $\rho$ is the density of the specimen, $u_{i}$ is the particle displacement occurred in the $i$ direction, and $t$ is time. $\mu$ denotes the shear viscoelasticity and represents $G$ and $\eta . \nabla^{2} u_{i}$ is the spatial Laplacian of the particle displacement. $\omega_{S}\left(=2 \pi f_{S}\right)$ is the angular frequency of the $\mathrm{SW}$, and $j$ denotes the imaginary unit, where $j^{2}=-1$.

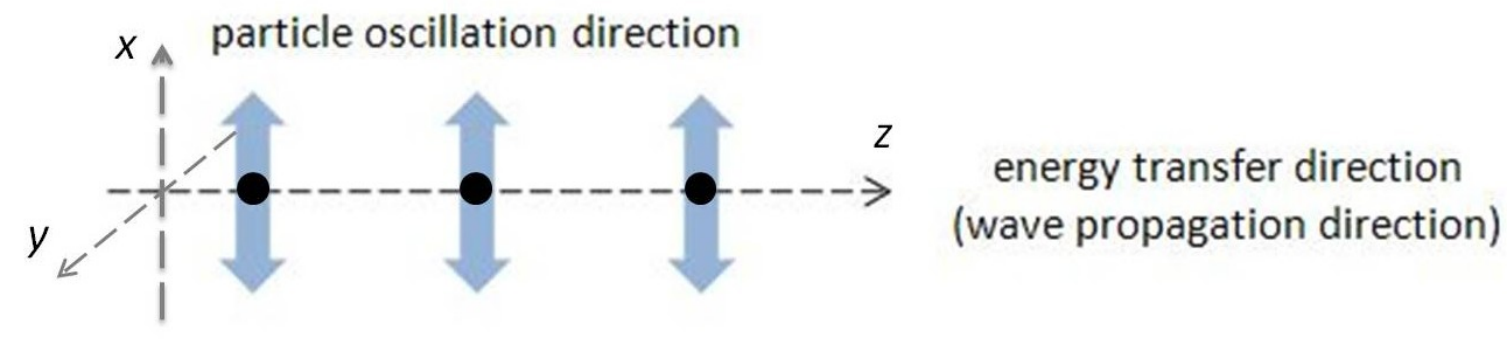

Shear Wave

Figure 2.3. Particle oscillation of shear wave.

When the specimen is homogeneous and isotropic, one of the solutions of the wave equation Eq. (2.1) is the plane SW. As shown in Fig. 2.3, assuming that particle displacement occurs only at $x$ direction $\left(u_{y}=u_{z}=0\right)$, thus the displacement, $u_{x}$, of the plane SW propagating along $z$-direction can be expressed as:

$$
u_{x}=A_{S} \sin \left(k_{S} z-\omega_{S} t\right)
$$

where $A_{S}$ is the amplitude, and $k_{S}\left(=\omega_{S} / v_{S}\right)$ is the wavenumber of the SW. 
Based on the Voigt model, the velocity and the absorption of the SW, $v_{S}$ and $\alpha_{S}$, respectively, have the following relationships with $G$ and $\eta[51]$ :

$$
\begin{aligned}
& v_{S}=\sqrt{\frac{2\left(G^{2}+\omega_{S}^{2} \eta^{2}\right)}{\rho\left(G+\sqrt{G^{2}+\omega_{S}^{2} \eta^{2}}\right)}} \\
& \alpha_{S}=-\sqrt{\frac{\rho \omega_{S}^{2}\left(\sqrt{G^{2}+\omega_{S}^{2} \eta^{2}}-G\right)}{2\left(G^{2}+\omega_{S}^{2} \eta^{2}\right)}} .
\end{aligned}
$$

Therefore, $G$ and $\eta$ can be determined by solving the Eqs. (2.4) and (2.5), as expressed by the following equations:

$$
\begin{aligned}
& G=\frac{\rho \omega_{S}^{2} v_{S}^{2} \cdot\left(\omega_{S}^{2}-\alpha_{S}^{2} v_{S}^{2}\right)}{\left(\omega_{S}^{2}+\alpha_{S}^{2} v_{S}^{2}\right)^{2}}, \\
& \eta=\frac{\rho \omega_{S}^{2} v_{S}^{2} \cdot 2 \alpha_{S} v_{S}}{\left(\omega_{S}^{2}+\alpha_{S}^{2} v_{S}^{2}\right)^{2}} .
\end{aligned}
$$

It is worth noting that for a soft tissue, its elastic property dominates the tissue mechanical property and the viscous property was often negligible. In such case, it was considered as a pure elastic material and $\alpha_{S}$ could be negligible. Then, Eq. (2.6) can be simplified to:

$$
G=\rho v_{S}^{2}
$$

When the $\eta$ was taken into account for tissue characterization, according to Eq. (2.7), $\eta$ can be determined either using both $v_{S}$ and $\alpha_{S}$, or alternatively by evaluating the dispersion of $v_{S}$ with respect to $\omega_{S}$ without the value of $\alpha_{S}$, which means, solving the Eq. (2.7) by knowing multiple pairs of $v_{S}$ and $\omega_{S}[6]$. 


\subsection{Notable Techniques of Viscoelasticity Measurement}

It was known that human has been using the sense of touch to attempt at diagnosis and/or treatment of some of the illnesses for thousands of years. For example, palpation has been used to detect breast tumors since 2,000 years ago [52]. Throughout the past century, modern medicine found that most of the tumors could be harder/stiffer than the surrounding normal tissues, and many research findings revealed that the viscoelastic properties could reflect the pathological information of tissues [10]. In addition to the pathology, as mentioned earlier in the previous section and shown in Fig. 2.1, the shear modulus of various normal tissue spans over orders of magnitude [41], potentially providing high contrast among different tissues/organs regarding their mechanical properties, which is something different from what most of the conventional medical anatomic imaging techniques could provide. Therefore, during the last three decades, medical research on human tissue viscoelasticity became more popular, as noninvasive and quantitative measurements of the viscoelastic properties of tissue can be very useful in medical diagnosis [1], [4], [52].

Through tens of years, many notable methods and techniques have been proposed and developed to quantify and visualize the tissue viscoelasticity. Most of the developed methods were based on either ultrasound technique or magnetic resonance imaging (MRI) [8]-[25], [33]. Ultrasound technique typically used ultrasound transducers to collect the information of the compressive force or SW excitation response by means of the tissue displacement. In such technique, the field of view (FOV) was limited by the penetration depth of the ultrasound, thus the region of interest (ROI) at deeper area or for obese subjects may possibly not be reached [20]. As a contrast, MRI technique was not 
subjected to those limitations. Measurement of elasticity with MRI has shown promise in the detection and diagnosis of liver [27], [53]-[55], breast [45], [56], [57], prostate [8], [14], [58], [59], and brain [60], [61]. However, MRI required a relatively long acquisition time (more than $10 \mathrm{~min}$ ), and it was also expensive and weak in portability.

Ultrasound-based techniques were also very actively studied and developed over the years [26]-[33], [62]-[67]. With ultrasound-based techniques, methods of tissue characterization could be divided into two groups according to the temporal difference of the excitation: quasistatic measurement method and SW measurement method.

Temporally, a quasistatic measurement was implemented at two relatively static steps: one without the employment of compressive force, and another with it. The schematic and concept of this measurement method can be seen in Fig. 2.4. Assuming the subject specimen was an elastic spring with an original length of $l$, when compressive force was applied onto the top of the spring, a displacement $\Delta l$ occurred along the axial direction due to the stress-strain behaviour, and the strain $\varepsilon$ was then defined by $\Delta l / l$. Both $\Delta l$ and $l$ can be obtained by an ultrasound probe posited at the top of the specimen. Since the stress $\sigma$ may not be measured in practice, the absolute value of $E$ may not be determined. Instead, a relative stiffness could be determined, i.e. higher $\varepsilon$ represented a softer tissue whereas lower $\varepsilon$ represented a stiffer tissue, as indicated in Fig. 2.4. J. Ophir and his colleagues in 1991 firstly proposed and described this quasistatic measurement method [1]. Considering that such method finded the elastic property of the tissue, it was named as "elastography" at that time. It was worth noting that, after a widely referred review paper by Gao et al. in 1996, the term "elastography" was widely expanded to describe all types of elasticity imaging [4]. It should be also noted that with a quasistatic 
measurement, tissue viscosity can also be determined by knowing the elasticity and measuring the relaxation time of the tissue from the compressive force [5], [68], [69].

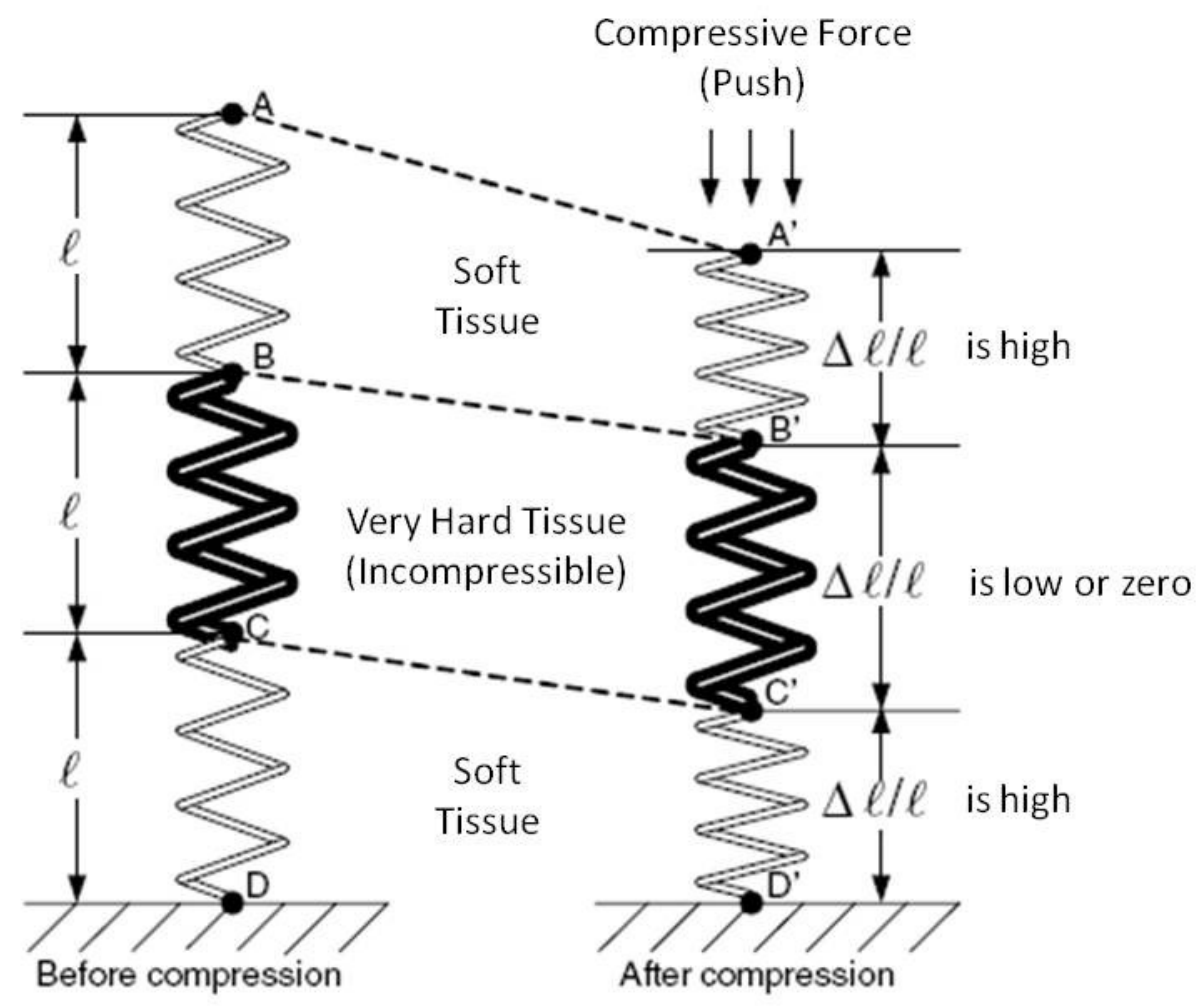

Figure 2.4. Schematic to explain the principle of compressional elastography (adopted from [45]).

The SW measurement method obtains the viscoelastic parameters according to Eqs. (2.1)-(2.8), by tracing and analyzing a SW propagation in a specimen of interest. In 1988, R. Lerner and K. Parker proposed a method to map the propagation of low frequency SW with a Doppler ultrasound displacement detection technique [70], and such image of SW displacement would reflect the tissue stiffness. Later in 1990, Y. Yamakoshi et al. proposed a dynamic measurement method to measure the $v_{S}$ using an external mechanical vibration source [7]. A configuration of this method can be seen in 
Fig. 2.5. In this method, $v_{S}$ could be determined by analyzing the wavelength of a continuously propagated SW through the ultrasound Doppler technique.

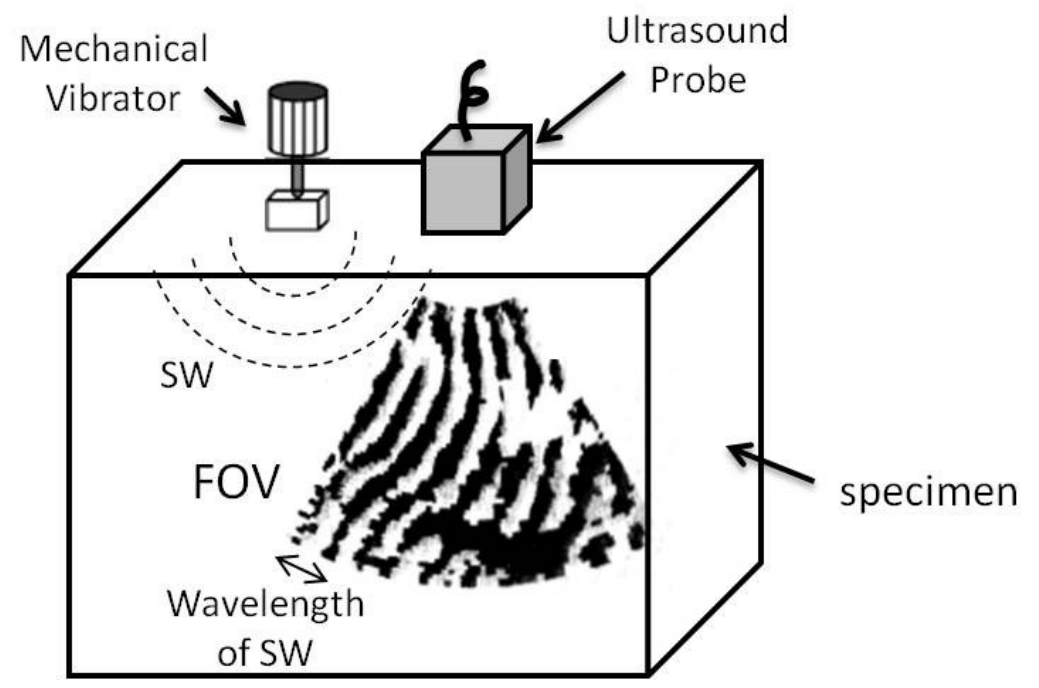

Figure 2.5. Experimental configuration of Yamakoshi's method (reproduced from [7]).

Instead of measuring continuous SW, Catheline and M. Fink developed an impulse SW measurement method in 1999, which used a fast frame rate plane wave ultrasound imaging, to trace the wave front of an impulse SW [71]. In this method, an ultrasonic probe was located at one side of the specimen to capture the propagation of the impulse SW that was generated by mechanical vibrator located at the other side of the specimen, using a plane wave ultrasound system. A conventional focused ultrasound system triggered ultrasound transducers sequentially from one edge of the linear array transducers to another for B-mode scan in which a frame rate was typically less than 100 frame/sec. On the other hand, a plane wave ultrasound system triggered all the ultrasound transducers at the same time to emit a plane ultrasound wave, enabling it to have a high frame rate (more than 1,000 frame/sec) of B-mode measurement. The $v_{S}$ was determined through a time-of-flight technique by measuring the time elapse of the impulse SW 
traveling through two locations with a known distance. Since such a method used impulse SW, it was also called transient SW imaging, or transient elastography. The transient SW measurement using a plane wave ultrasound system required intensive signal processing, making the overall cost of such system more expensive than that of a conventional focused ultrasound system.

Another notable SW measurement method was developed in 2004 by the group of K. Parker [34]. In this method, two SW sources located at two opposite sides of the specimen generated continuous SWs toward each other with slightly different SW frequencies, as shown in Fig. 2.6. Due to the frequency difference, the interfered SW patterns moved with a much slower speed than its actual $v_{S}$. Thus such a moving pattern was termed as a "crawling wave". Since the movement of the crawling wave was significantly slower than $v_{S}$, it was able to be observed with a conventional low frame rate B-mode scanning system. Once the speed of the crawling wave was obtained, the $v_{S}$ can be calculated.

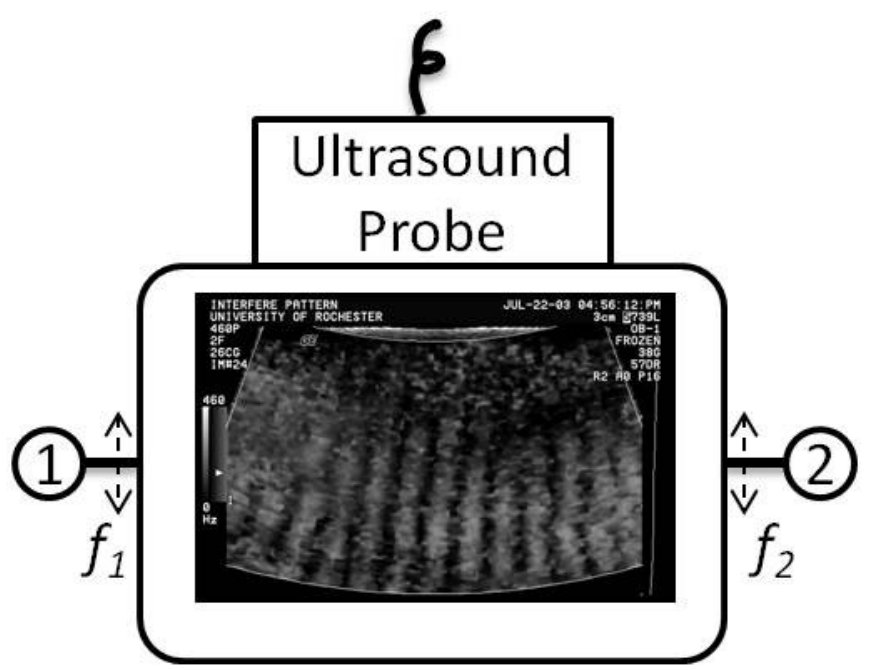

Figure 2.6. Experimental configuration for measurement of crawling wave, (1) and (2) are vibrators (reproduced from [34]). 
The SW measurement methods mentioned above use the external vibration source(s) to generate a SW propagating in the specimen. The SW excitation can be also conducted at the inside of the specimen by means of an ultrasound radiation force (or acoustic radiation force, ARF) technique [10]. Many creative methods of elastography have been developed based on the use of the ARF technique.

In 1998, Sarvazyan et al. developed a SW measurement method, termed shear wave elasticity imaging (SWEI), by using a SW remotely generated by the ARF of a focused ultrasound beam [10], as can be seen in Fig. 2.7. In this method, a transient SW pulse was firstly produced at the focus of the ultrasound beam and propagated along sideways (perpendicularly to the axis of the focused ultrasound beam). Then, imaging transducers were used to trace the moving of the SW wavefront and viscoelastic parameters can be derived thereafter. In 1998, Fatemi and Greenleaf developed a method to produce an oscillatory ARF by mixing two ultrasound beams with different frequencies [11]. A harmonic (continuous) or tone-burst SW was generated and propagates along sideways in this method, which made the SW narrow-band (while the SW generated by Sarvazyan's method was broadband) and then $v_{S}$ can be determined by finding the phase difference between the SWs at two apart locations [17]. By repeating the measurement with continuous SWs at different frequencies, or retrieving different frequency components of a tone-burst SW, both $G$ and $\eta$ can be derived according to Eqs. (2.6)-(2.7). Greenleaf later termed this method as SW dispersion ultrasound vibrometry (SDUV) [20], and its measurement configuration was illustrated in Fig. 2.8. 


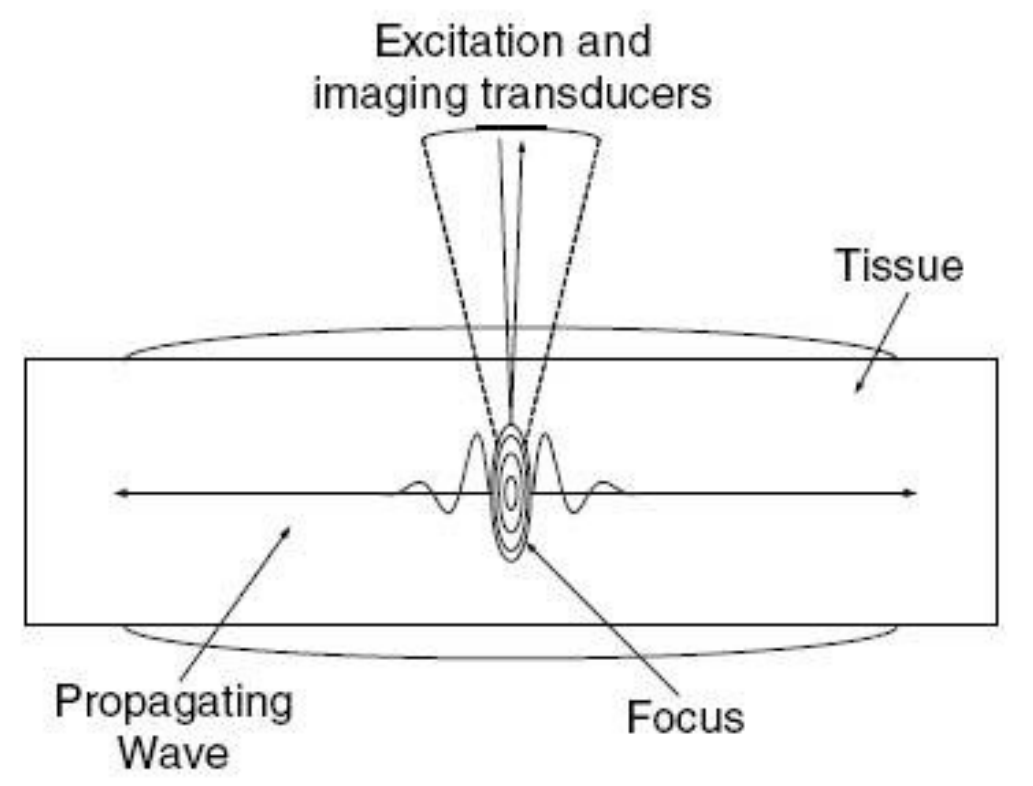

Figure 2.7. Schematic concept of acoustic radiation force to excite a transient SW (adopted from [45]).

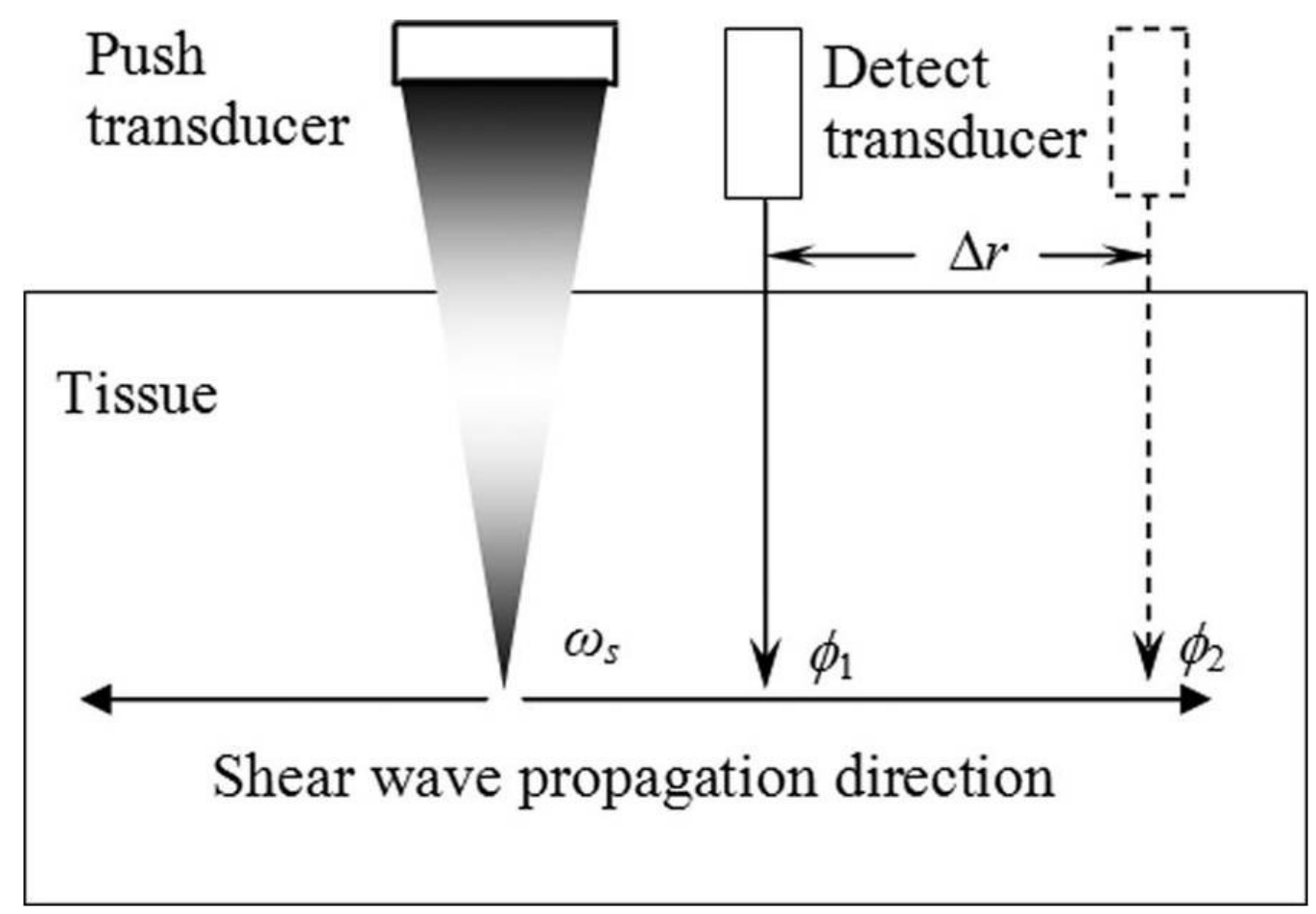

Figure 2.8. Measurement configuration of SDUV (reproduced from [20]). 
In 2001, Nightingale et al. introduced a different measurement modality termed as acoustic radiation force imaging (ARFI) [72]. Unlike other SW measurement methods which generated and analyzed SW, the ARF has been used to push and displace local tissues in ARFI technique. Depending on the stiffness of the tissue, the maximum displacement, and/or the relaxation time of the pushed tissue may be varying, and those parameters reflected the tissue elasticity. Because the principle of such technique was similar to a quasistatic measurement strategy, it was also called a remote palpation.

Later in 2004, based on the combination of Catherine's impulse SW method [71] and the ARF technique [10], Bercoff et al. developed an advanced SW measurement method which was termed as supersonic shear imaging (SSI) [16]. In this method, an ultra high speed scanner was used (could reach a frame rate at 10,000 frames/second), then multiple APF impulses excited very quickly at different depths, and each excitation produced a SW that propagates along sideways. All resulting SWs interfered constructively and created two plane SWs propagating in opposite directions, as can be seen in Fig. 2.9. By using the ultra high speed scanner to trace these SWs, a two dimensional (2D) $v_{S}$ image can be obtained. Moreover, since this technique created broadband SWs, tissue viscosity can be estimated through the $v_{S}$ dispersion method (using the same principle as SDUV).

Another ARF-based method was proposed in 2007 by McAleavey et al., named as spatially modulated ultrasound radiation force (SMURF) [50]. This method excited multiple ARFs together along a lateral direction (called comb-push excitation) to generate a SW at a desired wavelength $\left(\lambda_{S}\right)$. So the $v_{S}$ can be calculated through $v_{S}=\lambda_{S} / f_{S}$, by measuring $f_{S}$ in temporal domain. 

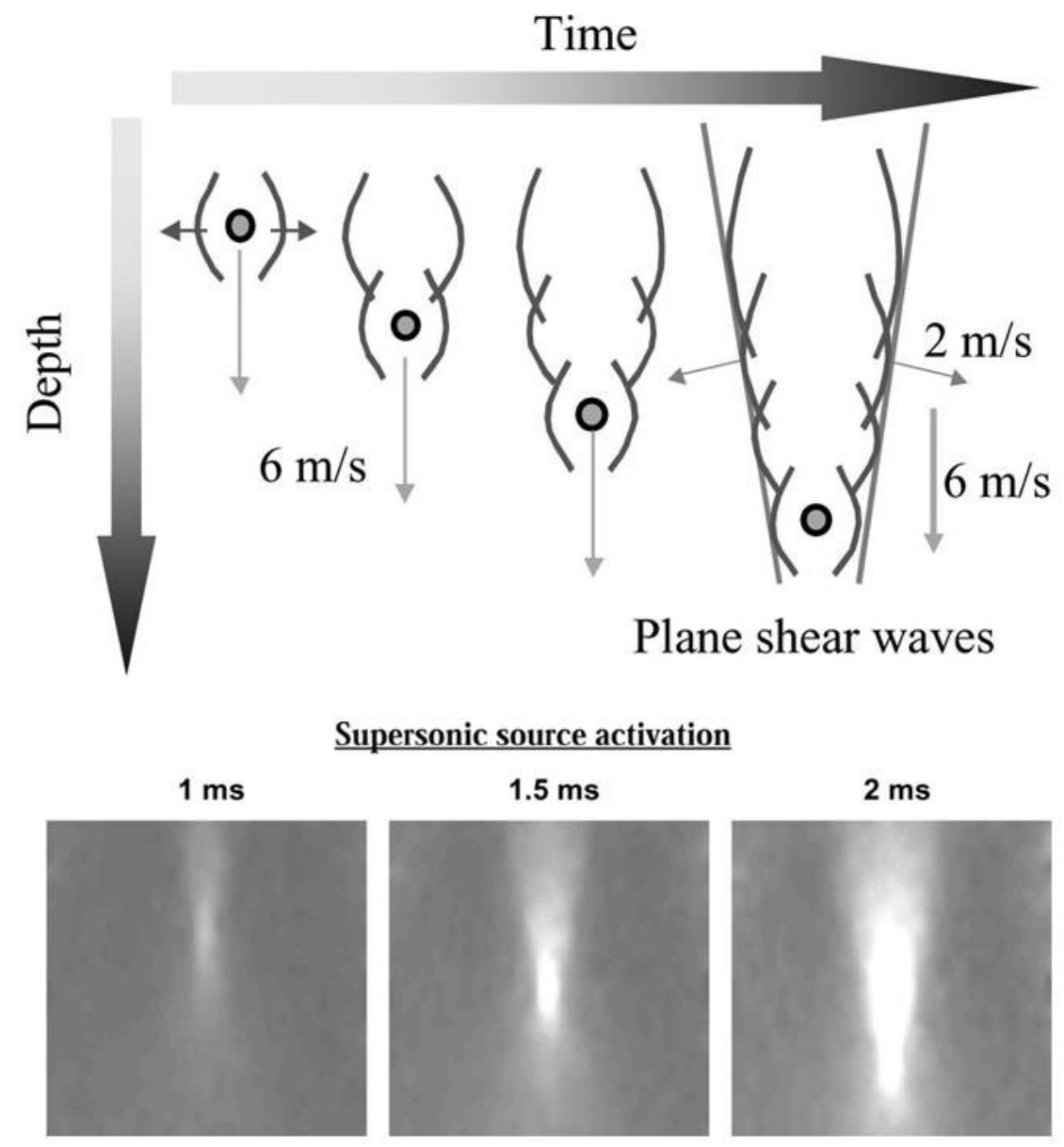

Plane shear wave propagation imaging

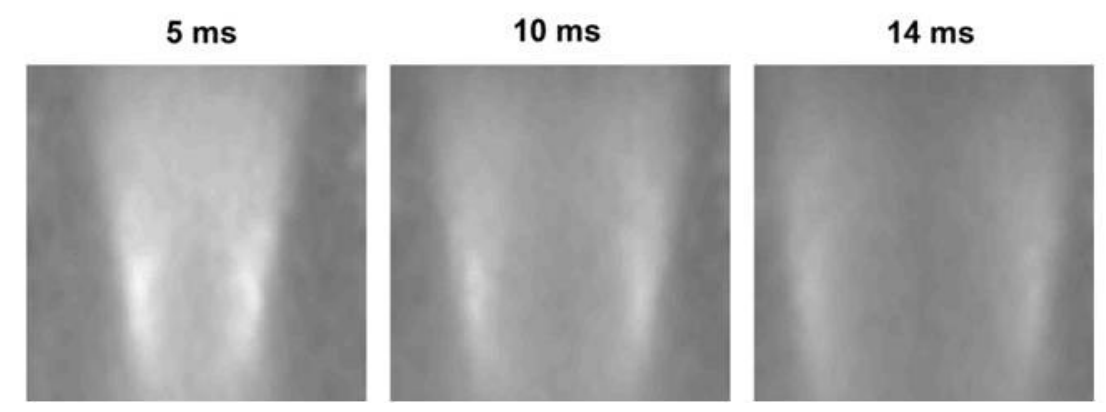

Figure 2.9. Generation of the supersonic plane shear wave (adopted from[16]).

More recently (in 2012), Greenleaf and his team developed a SW method which also used the comb-push excitation. This measurement method was termed as comb-push ultrasound shear elastography (CUSE) [24], [73], [74]. A schematic of the measurement configuration and observed SW patterns were illustrated in Fig. 2.10. It firstly generated 
multiple unfocused ARF excitations at different spatial locations to generate multiple SWs by using push mode of the ultrasound probe, and then quickly switched to the scanning mode of the probe to detect the SW propagation. Therefore the $v_{S}$ would be measured through the time-of-flight technique by tracing the movement of SW wavefront (as indicated by the arrows at different moments in Fig. 2.10) from each SW source. The use of comb-push excitation provided multiple SW sources in the specimen so that such method effectively compensated the worse signal-to-noise ratio (SNR) due to SW attenuation at the location far away from the single SW source.

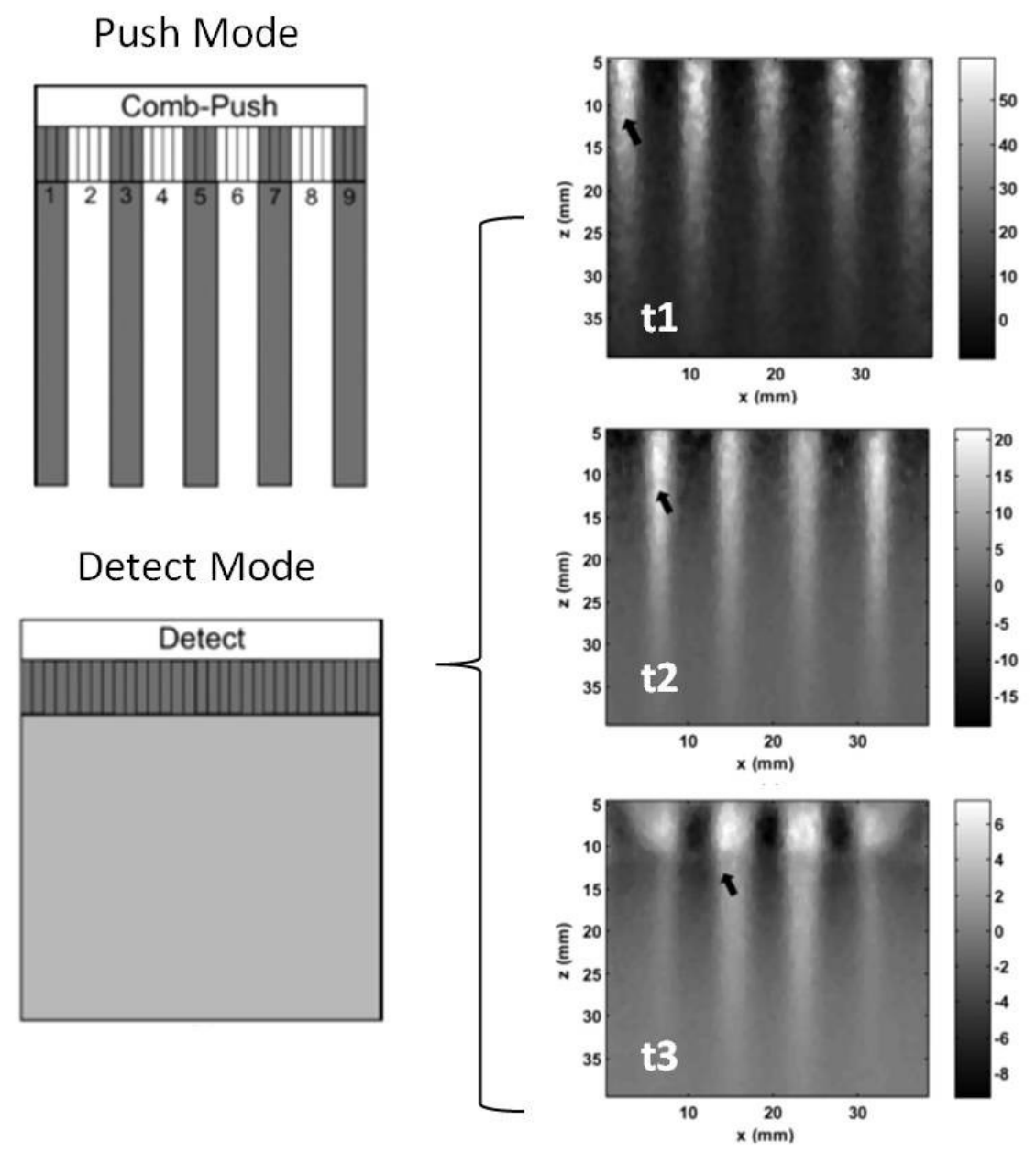

Figure 2.10. A schematic of the measurement configuration and observed SW patterns of CUSE (reproduced from [24]). 
By far, many studies have proved that the SSI technique may provide the most promising result among all the methods. Such technique was less affected by the problems stated in Section 1.2, and it has been generally acknowledged as the most advanced technique at present, in the field of ultrasound tissue characterization. The drawback of this technique could lie in the complexity of the system design and its expensive cost.

\subsection{Clinical Applications with Ultrasound Technique}

Over the past several years, many characterization methods have been applied into clinical applications. The techniques have already been adopted by the clinicians on diagnosis of several organs [45], [75]. Liver elastography was mainly used for the evaluation of liver stiffness to diagnose the patients with hepatitis (e.g. liver fibrosis and steatosis) [76]. A limitation lay in the depth of penetration, which could result in unsuccessful measurement with large patients or patients with ascites [45]. As a contrast, measurement at superficial regions could be more effective, such as the diagnosis of breast lesions and tumors. Both quasistatic [77] and dynamic [78] measurements were applied to detect the lesions in breast.

Diagnosis on prostate using elastography was a good complementary method to detect the potential cancer area [58]. When the lesion was identified, elastography may help the clinicians to make decision on whether to carry out a biopsy in the lesion area. Besides, elastography in thyroid was also employed to differentiate the cancers by evaluating the tissue stiffness [63]. Other applications, such as the characterization with 
musculoskeletal [65], Achilles tendon [79], cardiovascular [80], and lymph nodes [81] also showed good results that reflected the difference between normal and abnormal tissues. Moreover, some studies also pointed out that the measurement of elastic property could also be adopted to monitor the thermal therapy [82]-[84].

Many of the SW measurement methods have already been implemented in the commercial devices. Some of the typical devices are introduced here:

Echosens' FibroScan (France) - It is a machine based on transient elastography technique, which has been used in the detection of liver stiffness and used in Canada since 2009 [26], [76], [85].

SuperSonic Imagine's Aixplorer (France) - It is a machine based on the SSI technique, which has been widely used in many applications as introduced in the previous paragraph [30], [73], [79], [82], [86].

Siemens' Virtual Touch Quantification products (Germany) - Many modes are available, such as ACUSON NX3, ACUSON S2000, and ACUSON S3000. Those machines are based on the ARFI technique, which can be applied to liver, breast, thyroid, and abdominal [78], [87], [88].

General Electric (GE)'s LOGIQ E9 (US) - It is a machine that generates combpush ARFs and estimates $v_{S}$ from each ARF through the time-of-flight technique, which can be used to detect liver disease and breast lesions [73], [89].

At present, the improvement of measurement precision and accuracy of existing techniques was always an interesting and active topic. In addition, the most discussed parameter in the clinical applications was the tissue elasticity, while the measurement of 
viscosity was relatively less discussed. Therefore, the development of the comprehensive measurement of human soft tissue characteristics, such as viscosity, anisotropy, nonlinearity, and etc., could be the interesting research direction for the field of tissue characterization in the future. 


\section{Chapter 3}

\section{Development of Shear Wave Velocity Measurement}

\section{Method Using Focused Ultrasound and External Shear}

\section{Wave Source}

\subsection{Introduction}

When an induced SW was reflected from the tissue boundary, the observed SW could be an interference waveform. This may affect the measurement accuracy of the SW velocity, since the amplitude and the phase of the reflected SW may be unknown. Influence due to the reflection can be reduced by applying a directional filter at a spatio-temporal domain of the observed SW by using a plane wave ultrasound imaging system [35]-[37]. However, the use of a plane wave ultrasound did not meet the objective of this research (described in Section 1.3). Therefore, in this chapter a measurement method of SW velocity was proposed using a conventional B-mode scan of a focused ultrasound system with a single external SW source. First, the SW measurement model and ultrasound rf signal analysis procedure were presented. Then, the proposed method was verified experimentally by using a soft tissue mimicking phantom specimen. 


\subsection{Methodology}

An ultrasound brightness mode (B-mode) scan was composed of multiple sequential amplitude mode (A-mode) signals. A procedure was shown in Fig. 3.1. For most of the conventional ultrasound systems using a focused ultrasound, multiple ultrasound transducers were assembled as a linear array in an ultrasound probe. A focused ultrasound beam was formed by several consecutive activated transducers. Such beam would be transmitted into a specimen that the probe surface contacts, and ultrasound signals reflected from the inside of the specimen were received by those transducers. This procedure was defined as one A-mode scan. A-mode scan would be repeated sequentially from the one edge of the array transducer to another. The multiple consecutive A-mode scans formed a 2D image, defined as a B-mode image. Therefore, when the ultrasound signals were acquired by a focused ultrasound scanning system in B-mode scan, there was a time delay among the sequential A-mode measurements [73].

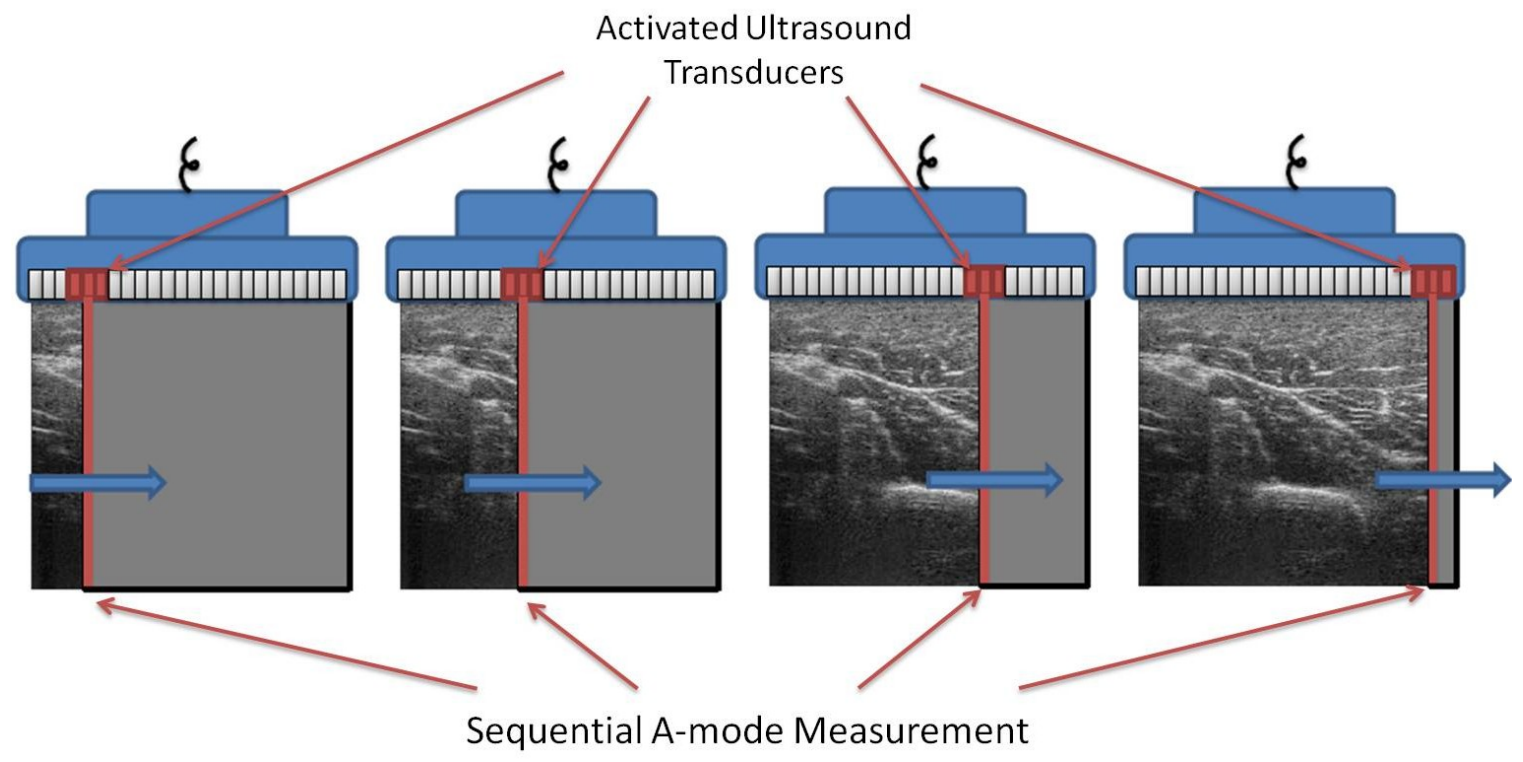

Figure 3.1. The procedure of B-mode scan using focused ultrasound. 
This scanning time delay caused an artificial spatial frequency shift in the observed SW propagating along the normal direction of focused ultrasound beam. The amount of the frequency shift depended on the propagation direction of SW with respect to the B-mode scanning direction. Therefore, forward and backward SWs could be separated from the observed SW using a spatial domain filter.

A measurement model of SW propagation using B-mode scan of focused ultrasound was depicted in Fig. 3.2. The origin of the coordinate system was selected at the center of the ultrasonic linear array probe and the SW source. The ultrasound probe was placed on top of the soft tissue specimen. In each A-mode measurement ultrasound rf signals were acquired along the depth ( $x$-direction) of the specimen.

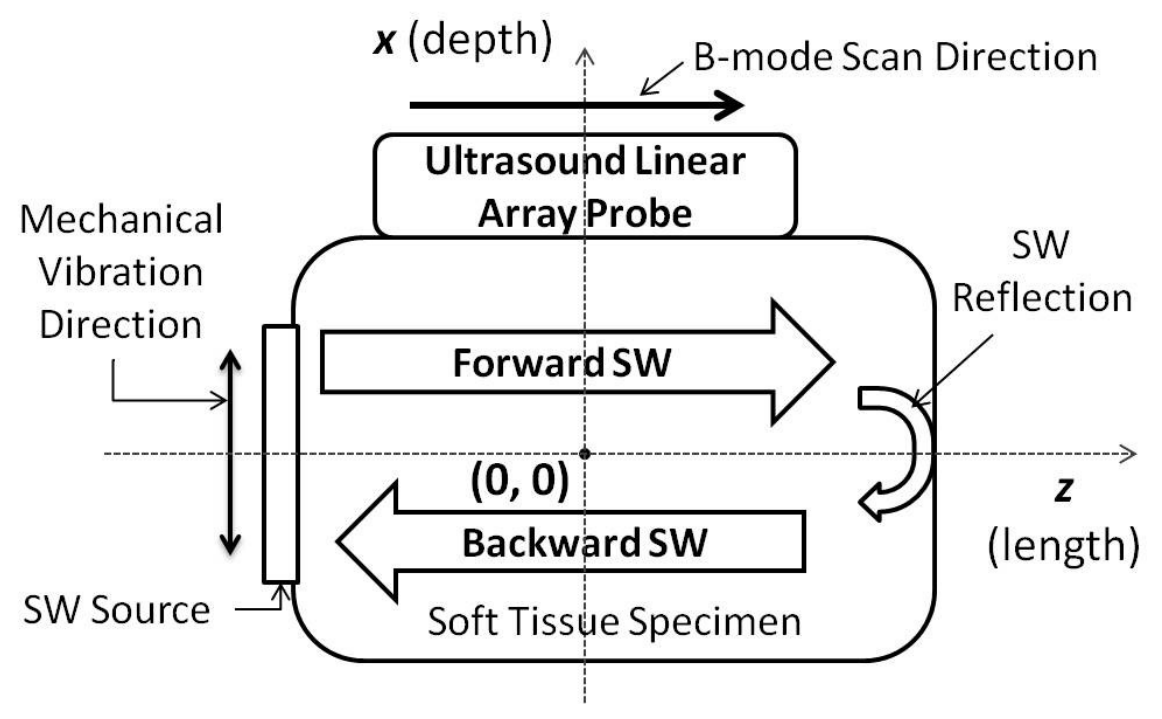

Figure 3.2. Measurement model of SW velocity.

A continuous SW vibration source contacted one side of the specimen. Thus, the SW propagated along the specimen length (z-direction) which was parallel to the scanning direction of the B-mode measurement. The induced SW was assumed to be a 
plane wave. The induced forward SW may be reflected from the opposite end or the boundaries in the specimen, resulting in a reflected backward SW. The backward SW could interfere with the forward SW. It was assumed that no SWs were converted into a longitudinal wave (LW) due to the mode conversion under the measurement model employed. For detailed explanation on mode conversion was given in Appendix A.

The tissue displacements in the specimen due to the SW propagation were obtained from the acquired ultrasound radio-frequency (rf) signals using a phase tracking method, and the SW displacement pattern in $z$-direction was observed. Then, the propagation velocity of SW was estimated from the phase variation of the observed SW. A flowchart of the ultrasound $\mathrm{rf}$ signal processing procedure to obtain the $\mathrm{SW}$ velocity, $v_{S}$, was depicted in Fig. 3.3.

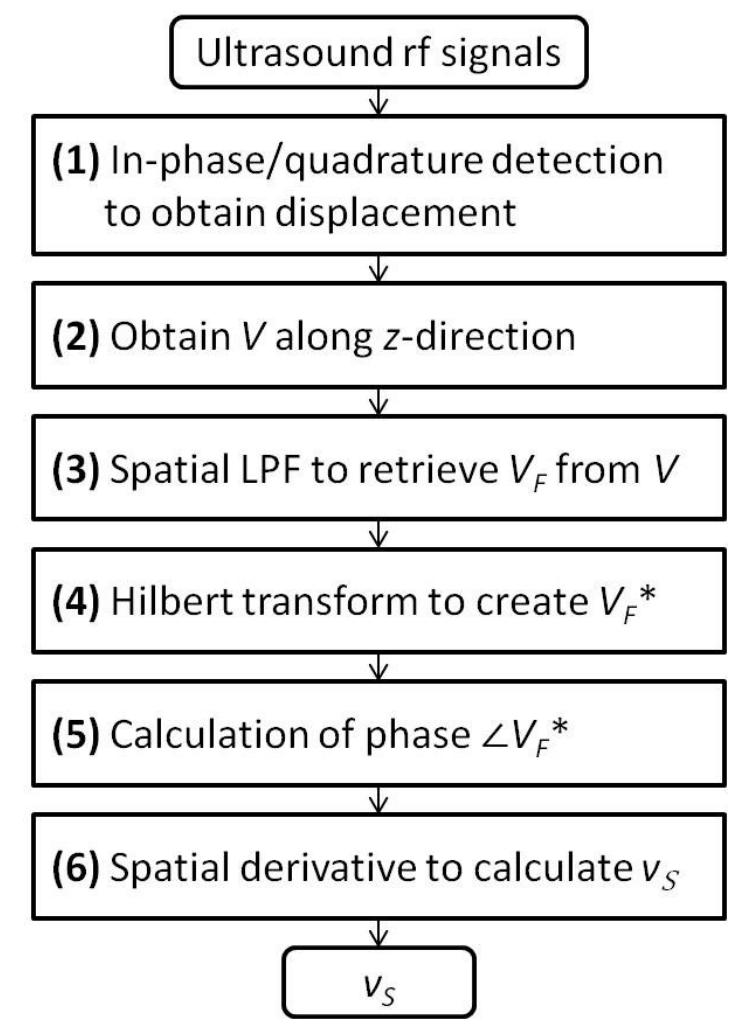

Figure 3.3. Flow chart of signal processing procedure for acquired ultrasound rf signals. 
The tissue displacements along the depth $x$ caused by the shear vibration were obtained by analyzing the phase of acquired ultrasound $\mathrm{rf}$ signals in each A-mode measurement [90], using an in-phase/quadrature detection technique (Step 1) [91]. Then, the waveform of SW displacement was obtained along the $z$-direction at a chosen depth $x$ (Step 2). For detailed signal processing procedures to obtain the SW displacement from rf signals was given in Appendix B.

The displacement of the observed SW, $V(t, z)$, was expressed by a summation of forward, $V_{F}(t, z)$, and backward, $V_{B}(t, z)$, SWs by Eq. (3.1), where $t$ was the time. By considering the B-mode scanning time delay, the $V_{F}(t, z)$ and $V_{B}(t, z)$ observed in B-mode measurement can be represented by Eqs. (3.2) and (3.3), respectively.

$$
\begin{aligned}
V(t, z) & =V_{F}(t, z)+V_{B}(t, z), \\
V_{F}(t, z) & =A_{F} \cos \left[\omega_{S}\left(t+\Delta t \frac{z}{\Delta z}\right)-k_{S} z+\varphi_{F}\right] \\
& =A_{F} \cos \left[\omega_{S} t-\left(k_{S}-\frac{\omega_{S} \Delta t}{\Delta z}\right) z+\varphi_{F}\right], \\
V_{B}(t, z) & =A_{B} \cos \left[\omega_{S}\left(t+\Delta t \frac{z}{\Delta z}\right)+k_{S} z+\varphi_{B}\right] \\
& =A_{B} \cos \left[\omega_{S} t+\left(k_{S}+\frac{\omega_{S} \Delta t}{\Delta z}\right) z+\varphi_{B}\right],
\end{aligned}
$$

where $A_{F}$ and $A_{B}$ are the $\mathrm{SW}$ amplitude, and $\varphi_{F}$ and $\varphi_{B}$ are the initial phase, of the forward and backward SWs, respectively. $\Delta t$ and $\Delta z$ are the temporal and spatial interval, respectively, between two consecutive A-mode measurements.

Due to the scanning time delay in the B-mode measurement, the time of each Amode measurement at the location $z$ had a delay, given by $\Delta t(z / \Delta z)$, from the first A-mode measurement. It resulted in the negative and positive artificial spatial frequency shift of 
$\omega_{S} \Delta t / \Delta z$ from $k_{S}$ for observed $V_{F}$ and $V_{B}$, respectively, as represented in Eqs. (3.2) and (3.3). Therefore, $V_{F}(t, z)$ can be retrieved from the measured $V(t, z)$, by using a low pass filter (LPF) in spatial domain (Step 3).

We defined the scan speed, $v_{\text {scan }}$, of the B-mode measurement by Eq. (3.4). Then, Eq. (3.2) may be rearranged to Eq. (3.5).

$$
\begin{aligned}
& v_{\text {scan }}=\frac{\Delta z}{\Delta t}, \\
& V_{F}(t, z)=A_{F} \cos \left[\omega_{S} t-\omega_{S}\left(\frac{1}{v_{S}}-\frac{1}{v_{s c a n}}\right) z+\varphi_{F}\right] .
\end{aligned}
$$

Therefore, it can be seen from Eq. (3.5) that the observed $V_{F}$ artificially moved backwards due to the scanning time delay when $v_{\text {scan }}<v_{S}$. Furthermore, the $V_{F}$ was observed with the same magnitude value at all positions of $z$ at a selected time $t$ when $v_{\text {scan }}=v_{S}$

An out-of-phase signal with $90^{\circ}$ phase shift for the $V_{F}$ at an arbitrary time $t$ with respect to $z$ was calculated by a Hilbert transform $\left(\mathcal{H}_{z}\right)$ to create an analytic complex signal $V_{F}^{*}$ as follows (Step 4):

$$
V_{F}^{*}(z)=V_{F}(z)+j \cdot \mathcal{H}_{z}\left\{V_{F}(z)\right\}
$$

where $j$ is the imaginary unit, which equals to $\sqrt{-1}$.

In the Hilbert transform of Eq. (3.6), we did not consider mathematically whether the observed $V_{F}$ moves forwards, or backwards artificially due to the scanning time delay. Therefore, the phase value of $V_{F}$ in Eq. (3.5) was obtained with the absolute value of the spatial phase component of $V_{F}^{*}$ using Eq. (3.7) (Step 5). By taking the spatial derivative of Eq. (3.7) with respect to $z, v_{S}$ at each $z$ was finally calculated using Eq. (3.8a) or 
(3.8b) (Step 6).

$$
\begin{aligned}
& \angle V_{F}^{*}(z)=\tan ^{-1}\left[\frac{\left[m\left[V_{F}^{*}(z)\right]\right.}{\operatorname{Re}\left[V_{F}^{*}(z)\right]}\right] \\
& =\omega_{S}\left|\frac{1}{v_{\text {scan }}}-\frac{1}{v_{S}}\right| z+\varphi_{F}, \\
& v_{S}(z)=\frac{1}{\frac{1}{v_{\text {scan }}}-\frac{1}{\omega_{S}} \cdot \frac{\partial\left[<V^{*} *(z)\right]}{\partial z}}, \quad \text { for } v_{\text {scan }}<v_{S} \\
& v_{S}(z)=\frac{1}{\frac{1}{v_{s c a n}}+\frac{1}{\omega_{S}} \cdot \frac{\partial\left[V_{F}^{*}(z)\right]}{\partial z}}, \quad \text { for } v_{\text {scan }}>v_{S}
\end{aligned}
$$

In order to measure the waveform of the $V_{F}$ along the B-mode scan direction (zdirection) without aliasing, the spatial sampling interval given by $\Delta z$ needed to satisfy the Nyquist sampling theorem expressed by Eq. (3.9). Therefore, the highest applicable $f_{S}$ was limited, as expressed by Eq. (3.10).

$$
\begin{aligned}
& \Delta z<\frac{1}{2} \cdot \frac{1}{f_{S}\left|\frac{1}{v_{\text {scan }}}-\frac{1}{v_{S}}\right|}, \\
& f_{S}<\frac{1}{2 \cdot \Delta z\left|\frac{1}{v_{\text {scan }}}-\frac{1}{v_{S}}\right|} .
\end{aligned}
$$

Figure 3.4 represented the highest $f_{S}$ with respect to $v_{S}$ under our measurement condition of $v_{\text {scan }}=2.26 \mathrm{~m} / \mathrm{s}$ (would be introduced in detail later). It was shown that the highest $f_{S}$ of $2847 \mathrm{~Hz}$ at $v_{S}=1 \mathrm{~m} / \mathrm{s}$ was the smallest in the $v_{S}$ range from 1 to $10 \mathrm{~m} / \mathrm{s}$. It was noted that most of SW measurements for tissue characterization were conducted at SW frequencies below $1000 \mathrm{~Hz}$ because of large attenuation of SW in biological soft tissues [7], [17], [21], [71]. In this chapter, $f_{S}$ of $500 \mathrm{~Hz}$ was chosen to demonstrate the feasibility of the proposed method. 


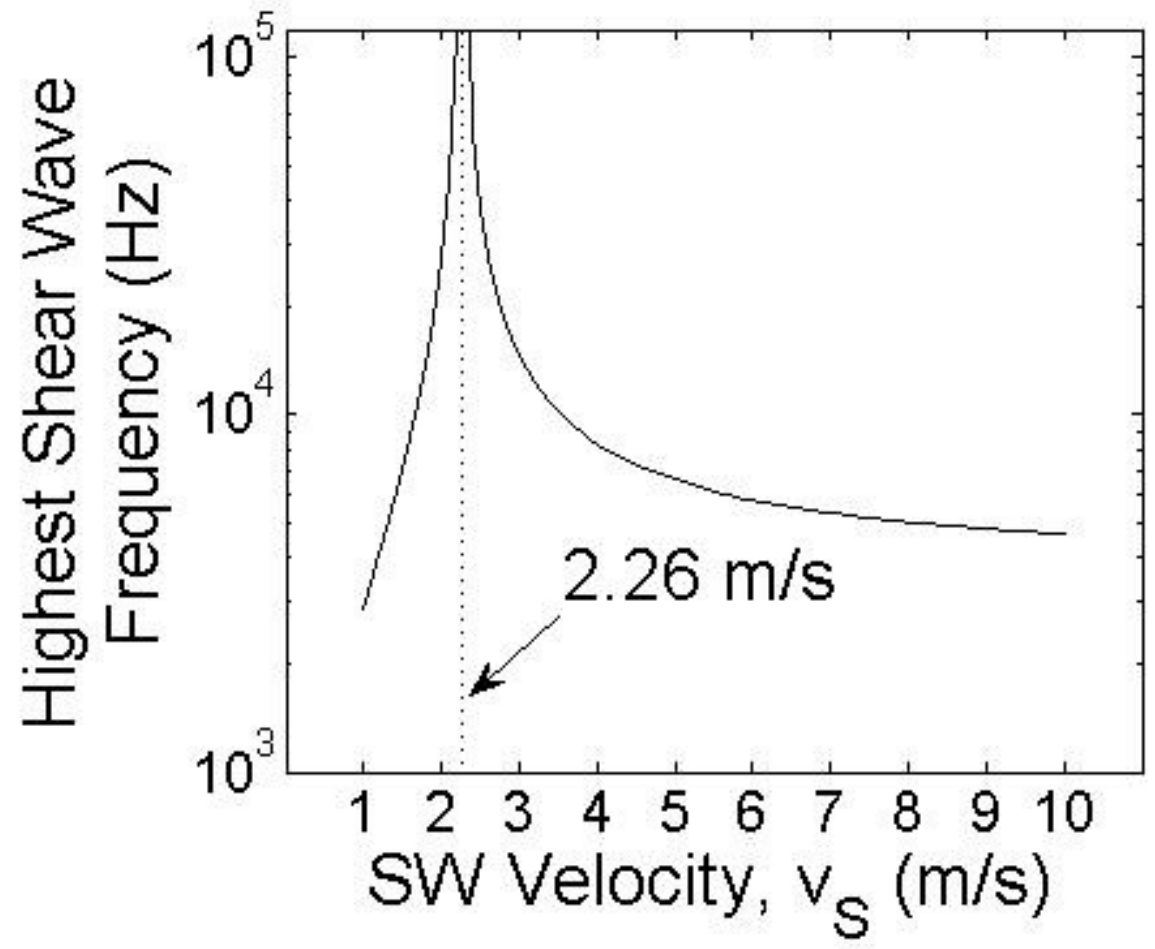

Figure 3.4. Highest SW frequency with respect to SW velocity to satisfy the Nyquist sampling theorem with ultrasound scanning system used in this chapter.

\subsection{Experimental Setup}

\subsubsection{Hardware Equipments and DAQ Program}

The hardware equipments used in the experiments shown in Fig. 3.5.

Ultrasound imaging system: the system model was a conventional B-mode scanning system (model: Picus) with a linear array ultrasound probe (model: L10-5, 5$\mathrm{MHz}$ nominal operating frequency). This equipment was from Esaote Europe, Maastricht, Netherlands.

Mechanical stage: The mechanical stage was built by a positioning device (Model: BiSlide, from VELMEX Inc., USA). It was moveable in three directions $(x, y$, 
and $z$ directions), and it was used to position the ultrasound probe onto a chosen location of a specimen.

External SW source: The SW source was assembled based on an acoustic speaker. An L-shape rigid plastic rod was permanently glued onto the diaphragm of the speaker, and a thin Plexiglas plate was mounted onto the other end of the rod. The Plexiglas plate was changeable, and it would contact onto the specimen and induce the SW into the specimen during the experiment.

Digital signal generator: Model: SFG-1003, from GW INSTEK, Taiwan. The digital signal generator was used to drive the acoustic speaker at a desired frequency.

Besides, an ART.LAB program was preinstalled in the ultrasound Picus system as a data acquisition (DAQ) program. It was used to monitor the B-mode or motion-mode (M-mode) image during the measurement and acquire the ultrasound rf signals as digital data.

There were 127 A-mode scan lines to form into a B-mode scan for our ultrasound probe. The spatial interval $(\Delta z)$ between two consecutive A-mode scan lines was $315 \mu \mathrm{m}$ and the ultrasound pulse repetition frequency (PRF) of A-mode scan was $7187 \mathrm{~Hz}$, from the manufacture report. The frame rate $\left(f_{F R}\right)$ of the B-mode scanning was also indicated at $30 \mathrm{~Hz}$. The exact values of the PRF and $f_{F R}$ were essential for the accurate $\mathrm{SW}$ measurement by the method proposed in this thesis. Therefore, in order to verify the value of PRF, the scanning time delay $(\Delta t=1 / \mathrm{PRF})$ between two consecutive A-mode scan lines was inspected as shown in Fig. 3.6. 


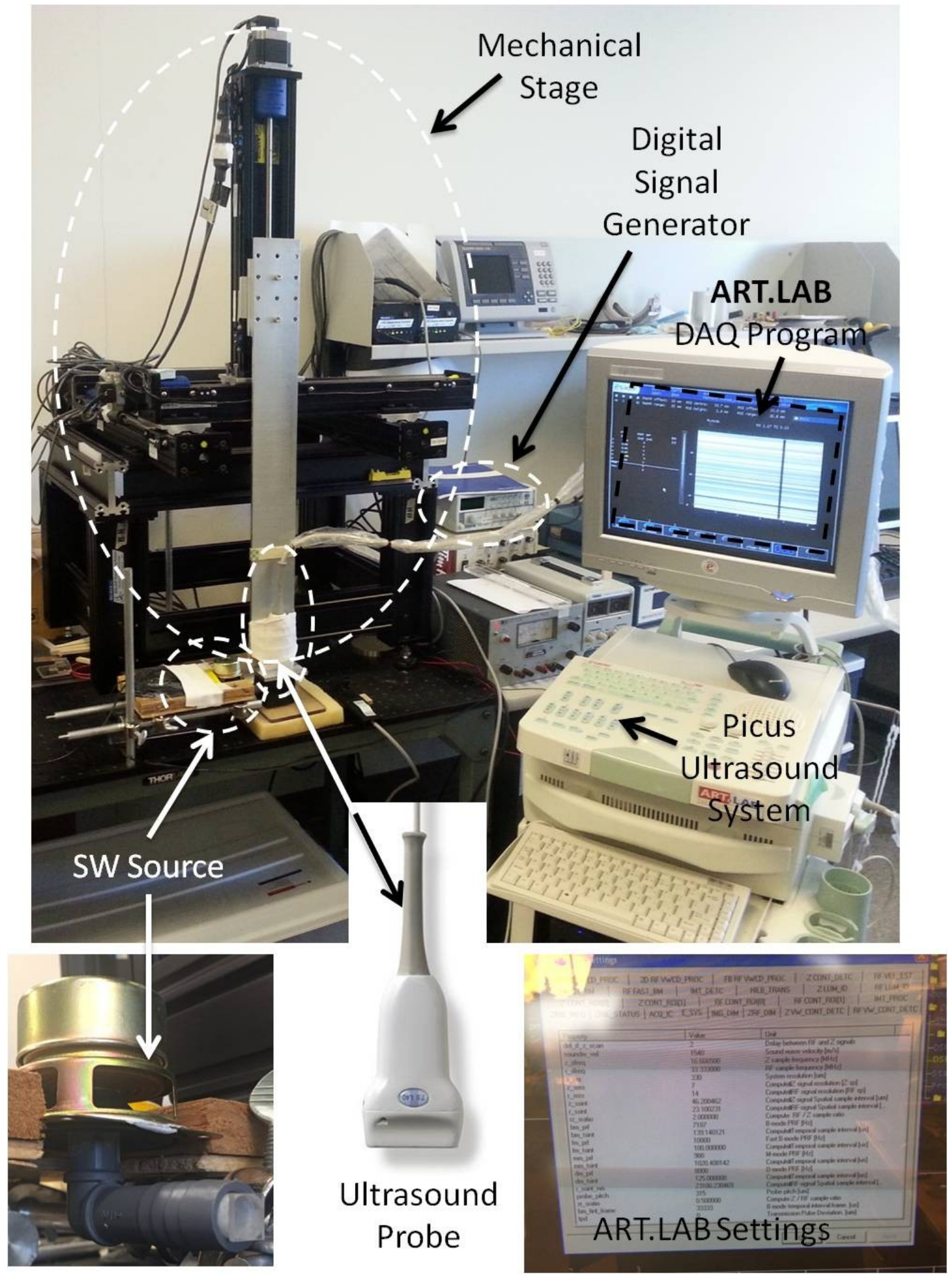

Figure 3.5. Equipments used in the experiment. 


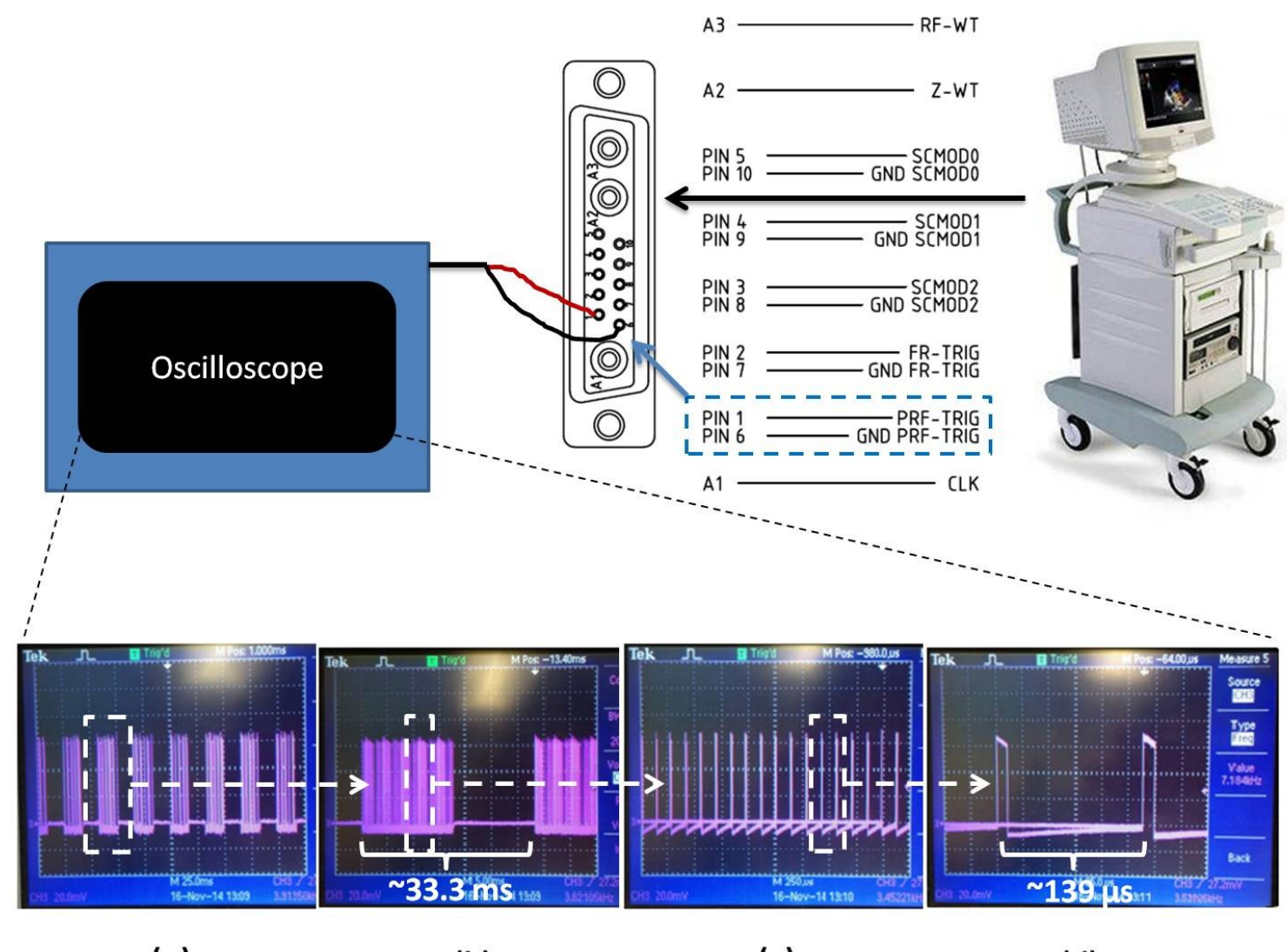

(a)

(b)

(c)

(d)

Figure 3.6. Verification of pulse repetition frequency and B-mode scan frame rate.

After the inspection, it was found each B-mode scan composed of 127 A-mode scans requires total $33.3 \mathrm{~ms}$ to be completed as seen in Fig. 3.6(b), thus $f_{F R}$ was verified $30 \mathrm{~Hz}(1 / 33.3 \mathrm{~ms})$. The duration between every two groups of excitations was considered to be the waiting time for signal processing and/or data archiving. The time interval between two consecutive single excitations was around $139 \mu$ s as seen in Fig. 3.6(d), thus the PRF value was $7187 \mathrm{~Hz}$ as indicated in the manufacture since $\Delta t$ was calculated at $139.1 \mu \mathrm{s}(1 / 7187 \mathrm{~Hz})$, which was very close to our result. Therefore, $v_{\text {scan }}$ of this ultrasound system in Eq. (3.4) was calculated $2.26 \mathrm{~m} / \mathrm{s}$. 


\subsubsection{Fabrication of Tissue-mimicking Phantom}

Phantom specimens were used in the experiments to verify the proposed SW measurement method. The phantom was hydrogel-based using either agar or gelatin to mimic soft tissues.

It took more than one day to accomplish the fabrication process of a hydrogel phantom. A general process was given as follows [92]:

1) Decided the concentration of the agar/gelatin in the phantom (which would determine the stiffness of the phantom fabricated), and weight the ingredients, such as agar/gelatin, graphite powder, alcohol and water, accordingly.

2) Prepared plenty of ice water for later use.

3) Boiled the water for around 30 minutes to degas it.

4) Kept on warming the boiled water to maintain the temperature above $90{ }^{\circ} \mathrm{C}$.

5) Stirred the water constantly (it is suggested to use a magnetic stirrer). Adjusted the stirring speed to avoid introducing air bubbles into the degassed water.

6) Mixed agar or gelatin powders into the water slowly to prevent them from forming into lumps.

7) Kept on stirring the solution until the powders were totally dissolved (powders were totally dissolved when the solution becomes transparent). Time consumption in this step would be subject to the amount and temperature of the solution. It may take tens of minutes to few hours. 
8) Mixed graphite particles into the solution slowly to prevent them from forming into lumps. The graphite particles were used as the ultrasound scatterers.

9) Kept on stirring the mixture for another hour to ensure the graphite particles being distributed uniformly.

10) Mixed more degassed heated water to compensate the loss due to evaporation. Controlled the water amount to maintain the solution at the desired agar/gelatin concentration.

11) Stopped heating the solution but kept on stirring it to avoid the sediment.

12) (Optional) Mixed certain amount of alcohol into the mixture to prevent the growth of bacteria to extend the lifetime of the phantom.

13) Kept on stirring the mixture until its temperature drops below $60^{\circ} \mathrm{C}$.

14) Got the phantom mould ready and let it be surrounded by the ice water (the ice water is prepared earlier as indicated in step 2). This step should be carried out a little earlier before the temperature of the mixture is below $60{ }^{\circ} \mathrm{C}$.

15) Stopped stirring the mixture when its temperature is below $60{ }^{\circ} \mathrm{C}$. Poured the mixture into the mould slowly to avoid introducing air bubbles.

16) Left the liquid phantom in the mould for at least an hour (ice water bath accelerates the solidification of the mixture).

17) Transferred the mould into the fridge from the ice water and waited for 24 hours to let it become totally solidified. 
18) Removed the solid phantom from the mould, and wrapped it with preservative film to minimize the evaporation of water component. Preserved the phantom in the fridge.

Every time before using the phantom for experiment, phantom would be taken out of the fridge for a few hours to let it reach the room temperature. This procedure was very important since the tissue viscoelastic properties may vary with the temperature.

Based on our experience, when alcohol was added, a hydrogel phantom which was made of agar could last for one to two months without changing its stiffness, and then it may start shrinking and hardening due to the water loss, while for a gelatin-based phantom, it may last for about two weeks. Yet when alcohol was not added, both phantoms may start molding and decaying within one to two weeks. Physically, an agar phantom seemed more durable than a gelatin phantom.

Note that it was relatively difficult to precisely adjust the acoustic properties, such as density, speed of sound, and attenuation, of the agar and gelatin phantoms. Therefore, the fabricated phantoms used in this thesis research were not adjusted to meet the recommended ultrasound properties for biological soft tissues (LW velocity around 1540 $\mathrm{m} / \mathrm{s}$, and attenuation around $0.5 \mathrm{~dB} \cdot \mathrm{cm}^{-1} \cdot \mathrm{MHz}^{-1}$ ) [93]. The objective of the phantom experiments was to verify the proposed SW measurement method, but not the fabrication of bio-tissue phantom. Furthermore, the proposed method measured the shear properties but not the longitudinal properties of a specimen. 


\subsection{Experiments}

Two soft tissue mimicking phantoms were fabricated through the process described in Section 3.3.2 for the experiments to verify the proposed method. One was constructed using 2-W\% agar, and the other was using 9-W\% gelatin. They were hydrogel phantoms with $2-\mathrm{V} \%$ isopropanol ( $99 \%$ purity) and 2-W\% fine graphite particles. The graphite particles applied had a nominal diameter of $50 \mu \mathrm{m}$. Both phantoms had a cubic shape with a side length of $50 \mathrm{~mm}$. Based on a tactile comparison, the gelatin phantom was softer than the agar phantom.

In order to characterize elastic properties of the fabricated phantoms, their mechanical parameters, such as Young's modulus, density, and LW velocity, were measured. First, stress-strain measurements (quasistatic method) were conducted to measure the Young's modulus. A weight of $200 \mathrm{~g}$ was applied uniformly on the entire top surface of the phantom, and the resulting thickness change of the phantom was measured by using a digital caliper with a resolution of $0.1 \mathrm{~mm}$. From the stress-strain analysis, the Young's modulus of the agar and gelatin phantom were obtained to be $78 \pm 16 \mathrm{kPa}$ and $49 \pm 6 \mathrm{kPa}$, respectively. The densities of both phantoms were $1.1 \mathrm{~g} / \mathrm{mL}$ calculated from the dimensions and weight of each phantom. Finally, the LW velocity was measured by using an ultrasonic pulse echo technique. A single element $4 \mathrm{MHz}$ ultrasonic probe was attached on the top surface of the phantom, and the first and second round-trip ultrasonic signals reflected from the bottom surface were acquired. The LW velocity of the agar phantom was $1467 \pm 1.4 \mathrm{~m} / \mathrm{s}$, and $1490 \pm 1.5 \mathrm{~m} / \mathrm{s}$ for the gelatin phantom.

The $v_{S}$ of each phantom can be estimated from the Young's modulus, LW velocity, 
and the density of a homogeneous isotropic material [94]. The $v_{S}$ was calculated $4.9 \mathrm{~m} / \mathrm{s}$ for agar phantom, and $3.9 \mathrm{~m} / \mathrm{s}$ for the gelatin phantom by using the measured elastic parameters presented above. The estimated $v_{S}$ of each phantom was greater than $v_{\text {scan }}$ $(2.26 \mathrm{~m} / \mathrm{s})$ of our ultrasound system. Thus, Eq. (3.8a) was used to obtained $v_{S}$ from the SW measurements with the phantoms.

After the measurement of elastic properties of each phantom, the two phantoms were jointed to mimic a heterogeneous material as shown in Fig. 3.7(a). One surface of each phantom was slightly melted with a heat source, then the melted surfaces were attached together. The combined phantom was cooled to solidify the joint boundary. Figure 3.7(b) presented a B-mode ultrasound image of the combined phantom. The vertical line at the center presented the interface of the agar and gelatin phantoms, and the horizontal line was the bottom echo.

The ART.LAB program was used to visualize the B-mode image and save the ultrasound rf signals. FOV of a B-mode scan was $40 \mathrm{~mm}$ in length (z-direction) and 34 $\mathrm{mm}$ in depth ( $x$-direction). In each measurement, a total of 180 frames of B-mode measurement were acquired in 6 seconds.

Two SW experiments were described in this chapter. In the first experiment, $v_{S}$ of each phantom (agar or gelatin) was measured with the proposed method. A $50 \mathrm{~mm} \times 50$ $\mathrm{mm}$ square Plexiglas plate with a thickness of $5 \mathrm{~mm}$ was contacted onto the side surface of the agar (or gelatin) phantom as the SW vibration source, and the ultrasonic probe was attached on the top surface of the selected phantom, as depicted in Fig. 3.8(a). The acoustic speaker was driven continuously by sinusoidal signals at $500 \mathrm{~Hz}$ during the experiments. Therefore a monochromatic SW was transmitted into the phantom, which 
induced vertical displacement of particles in the phantom.

In the second experiment, the SW source contacted the side surface of the agar phantom, as shown in Fig. 3.8(b), so that the SW could propagate in the entire specimen through the interface of the agar and gelatin phantoms. The ultrasonic probe was attached on the top surface of the interface so that the FOV could cover both regions of the agar and gelatin phantoms.

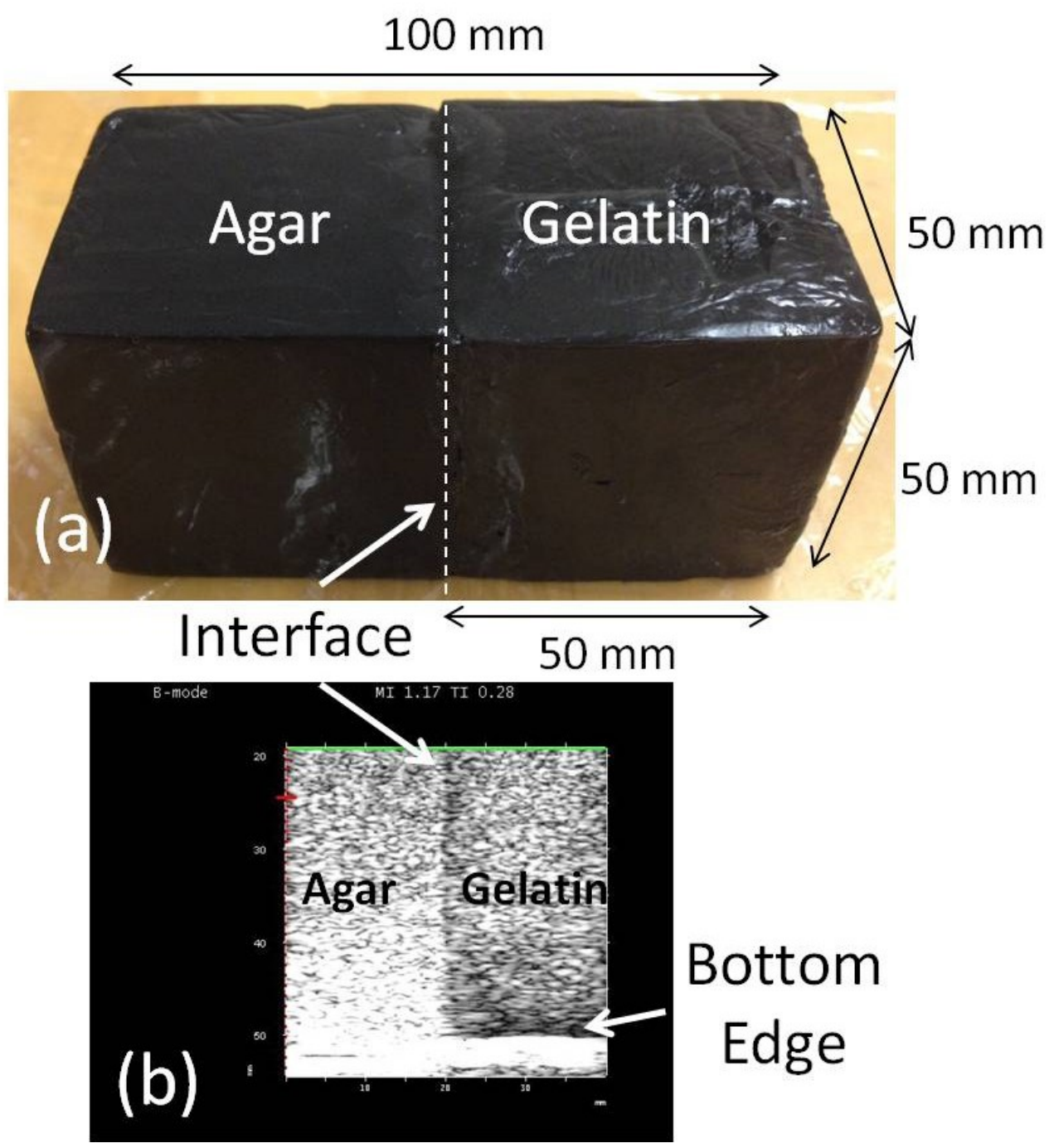

Figure 3.7. (a) Photo and (b) ultrasound B-mode image of a soft tissue mimicking phantom with combination of agar and gelatin phantoms used in the experiments. 


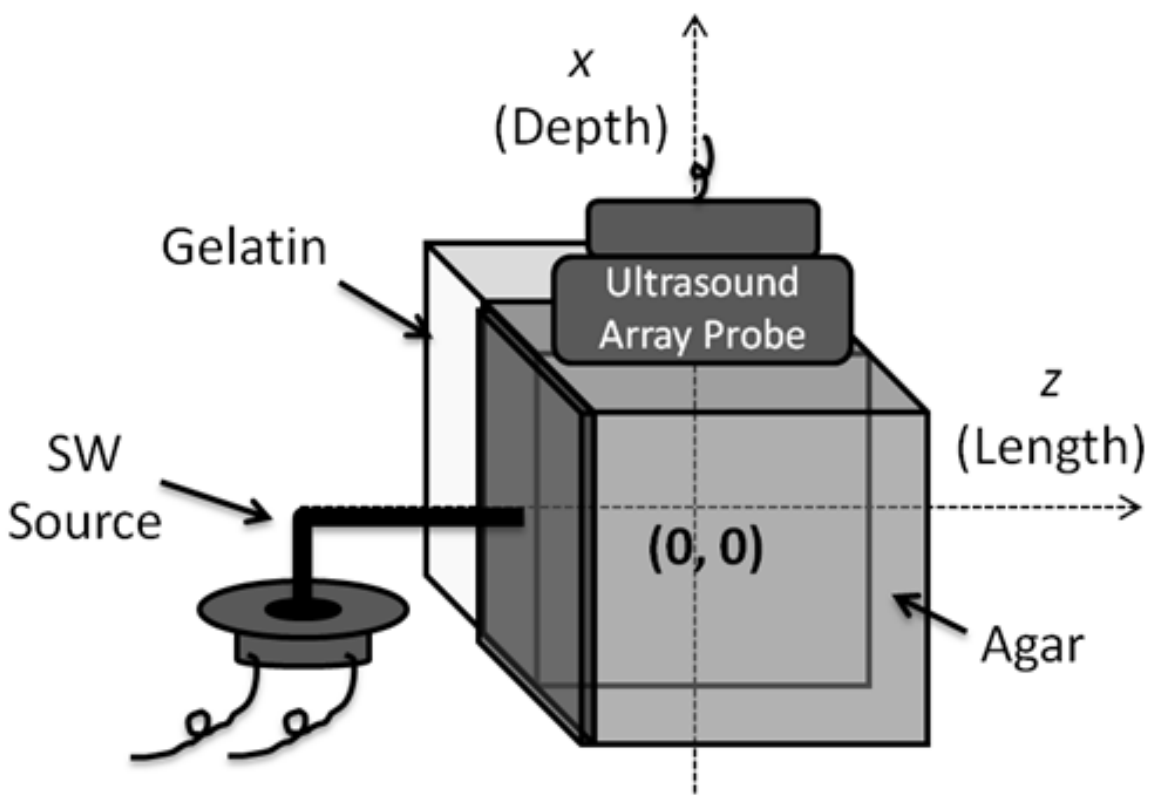

(a)

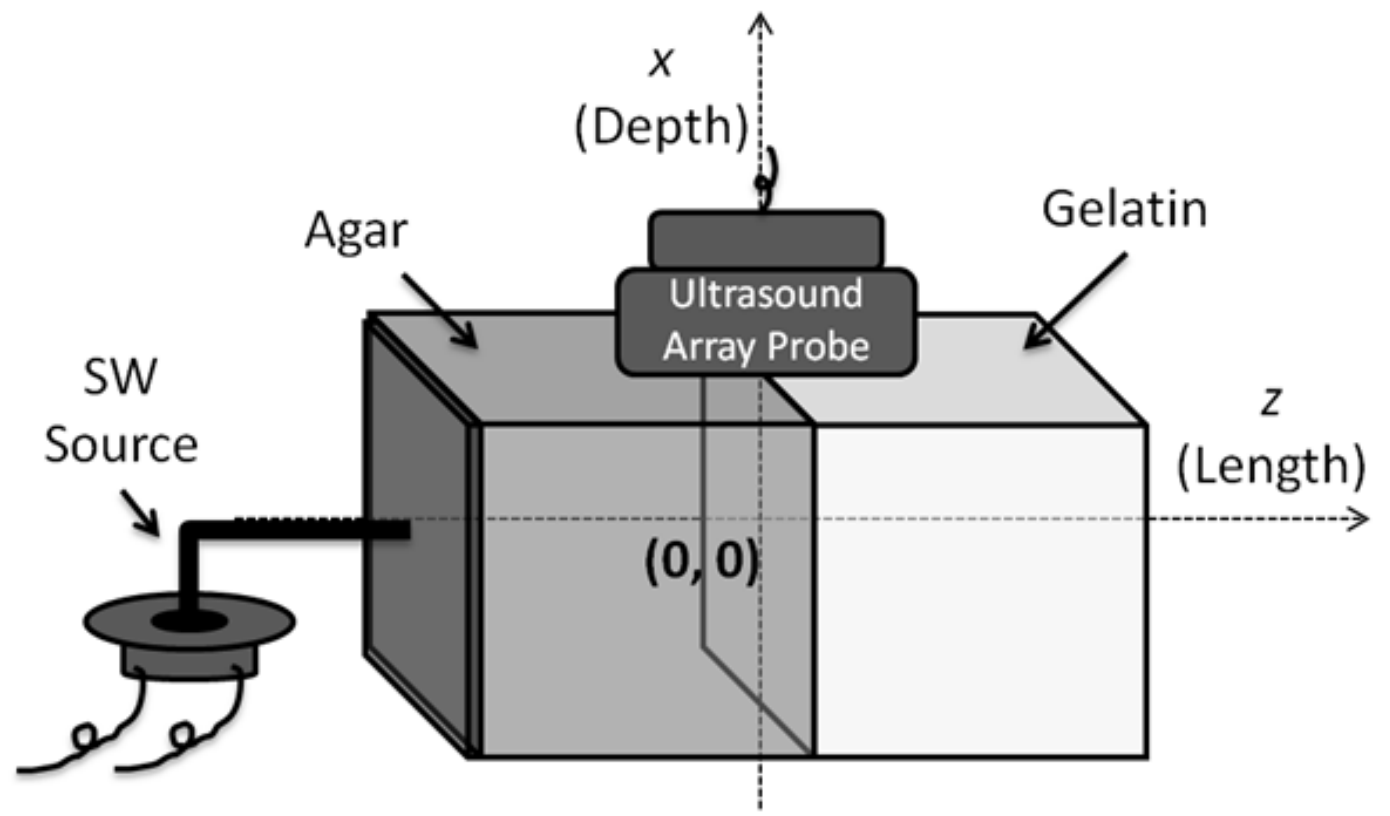

(b)

Figure 3.8. Experimental configuration of SW velocity measurement for (a) the individual phantom, and (b) the combined phantom. 


\subsection{Results and Discussions}

For the first experiment, the observed $\mathrm{SW}$ displacements, $V$, of the agar phantom were shown in Fig. 3.9(a). The displacement along $z$-direction at $x=0$ was also presented in Fig. 3.9(b), in which the interference of the forward $\left(V_{F}\right)$ and backward $\left(V_{B}\right)$ SWs was clearly observed as a fluctuation of the displacement from a sinusoidal-shape pattern. Figure 3.9(c) presented the spatial frequency spectrum of the measured SW displacement in Fig. 3.9(b). In Fig. 3.9(c), the two frequency components corresponding to the $V_{F}$ and $V_{B}$ were seen. The $V_{F}$ was retrieved from $V$ by the spatial LPF with a cutoff frequency of $1.4 \mathrm{rad} / \mathrm{mm}$, and the filtered results corresponding to the measured results in Figs. 3.9(a)(c) were shown in Figs. 3.9(d)-(f), respectively. It was noted that, in principle, $v_{S}$ can also be obtained through $V_{B}$ instead of $V_{F}$. However, $V_{F}$ was used because of its greater energy and better signal-to-noise ratio than $V_{B}$. The same measurement procedure and data analysis were performed for the gelatin phantom.

The result was averaged with 180 frames and was shown in Fig. 3.10(a). The $v_{S}$ result for the gelatin phantom was also shown in Fig. 3.10(b). The mean value of the $v_{S}$ in the FOV of the agar phantom was $5.9 \mathrm{~m} / \mathrm{s}$ with a standard deviation of $1.1 \mathrm{~m} / \mathrm{s}$, and that

of gelatin phantom was $3.3 \mathrm{~m} / \mathrm{s}$ with a standard deviation of $0.52 \mathrm{~m} / \mathrm{s}$. The measured $v_{S}$ of the agar phantom was $2.6 \mathrm{~m} / \mathrm{s}(78.8 \%)$ greater than that of gelatin phantom. 

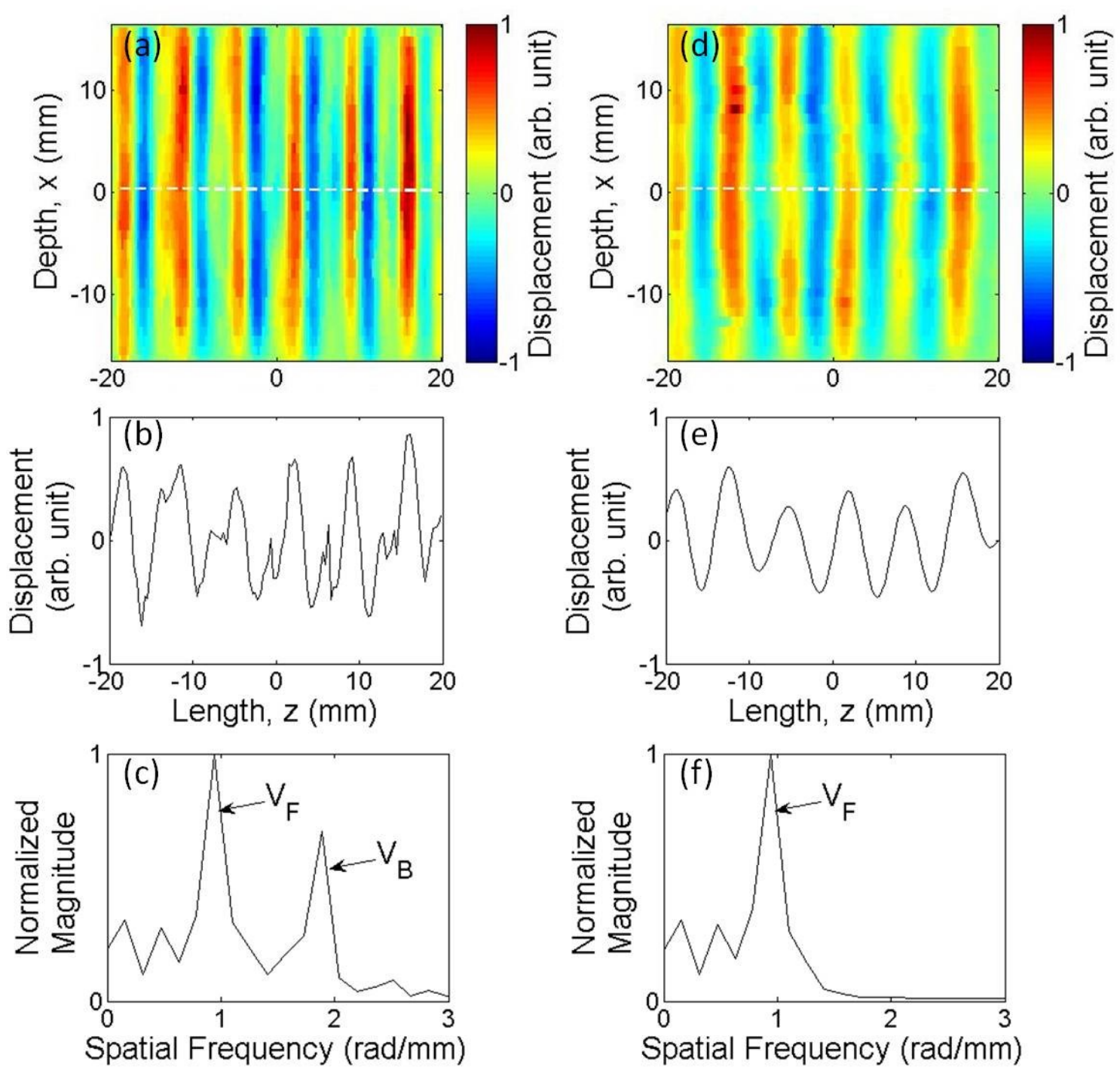

Figure 3.9. Observed SW displacements (a) in the entire FOV and (b) along $z$-direction at $x=0$, and (c) spatial frequency spectrum of the displacement shown in (b). Those of filtered SW displacements were presented in (d)-(f) respectively. 

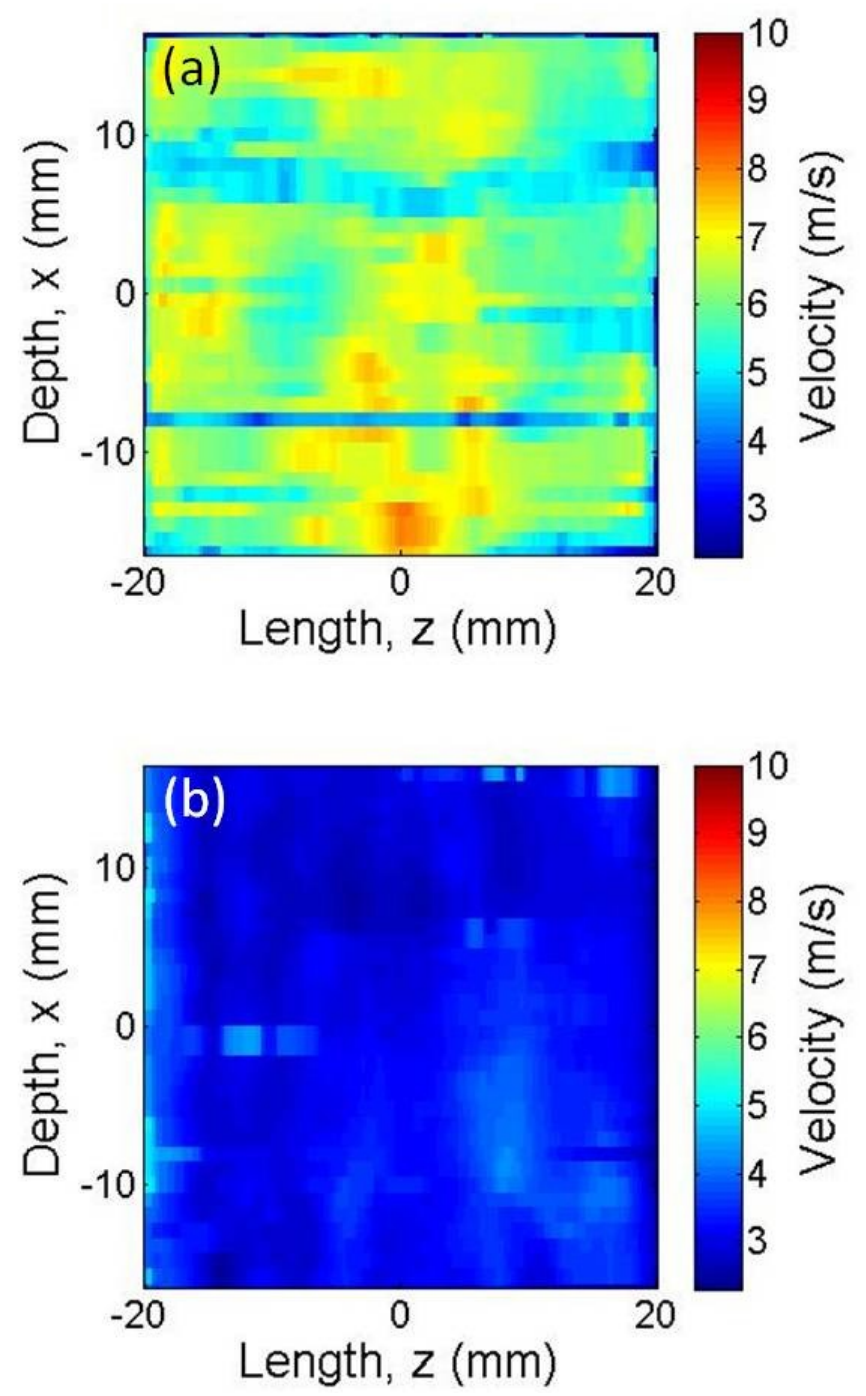

Figure 3.10. Measured SW velocity obtained from the filtered SW displacements of (a) the agar phantom and (b) the gelatin phantom.

In order to verify the reliability of the measurement results, the $v_{S}$ of both phantoms were also measured with an impulse technique [71]. An M-mode measurement with $935 \mathrm{~Hz}$ frame rate was conducted with the same ultrasound system to trace the propagation of an impulse SW induced by the SW source, and the configuration of such measurement can be seen from Fig. 3.11. Then, $v_{S}$ obtained from the impulse technique and calculated from the measured Young's modulus, LW velocity and density of the 
phantom were compared with $v_{S}$ obtained from the continuous SW measurement method proposed in this chapter. The results were summarized in Table 3.1.

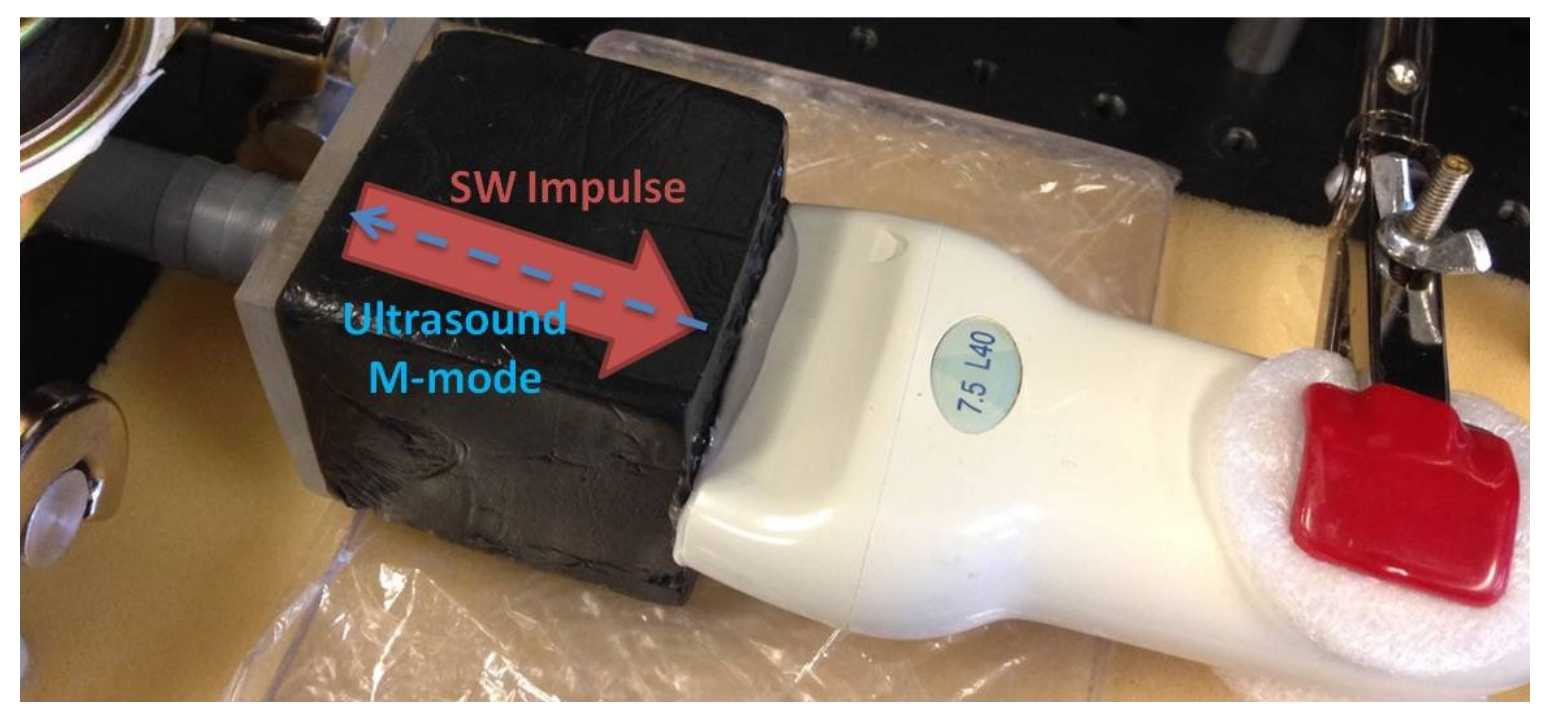

Figure 3.11. Configuration of the measurement on SW impulse using M-mode.

Table 3.1. A comparison of SW velocity obtained from different methods.

\begin{tabular}{|c|c|c|c|c|}
\hline \multirow{2}{*}{ Method } & \multicolumn{2}{|c|}{ SW velocity, $v_{S}(\mathrm{~m} / \mathbf{s})$} & \multicolumn{2}{c|}{$\begin{array}{c}\text { Difference from } \\
\text { proposed method }\end{array}$} \\
\cline { 2 - 5 } & Agar & Gelatin & Agar & Gelatin \\
\hline Proposed method & 5.9 & 3.3 & -- & --- \\
\hline Impulse technique & 6.0 & 2.9 & $1.7 \%$ & $-12.1 \%$ \\
\hline $\begin{array}{c}\text { Calculation with Young's } \\
\text { modulus, LW velocity, and } \\
\text { density }\end{array}$ & 4.9 & 3.9 & $-16.9 \%$ & $18.2 \%$ \\
\hline
\end{tabular}

Measurement results with the impulse technique had better agreement with the proposed method than the results obtained from the Young's modulus, especially for the agar phantom. This may be due to the fact that both the impulse technique and our proposed method were dynamic measurements of SW propagation. On the other hand, 
the estimation from the measured Young's modulus was based on static measurements.

The result of the $v_{S}$ measurement in the second experiment with the combined phantom was shown in Fig. 3.12. The interface of the phantoms was seen at $z=0$ where a sharp change of the $v_{S}$ due to the stiffness difference between the agar and gelatin phantoms was clearly observed. The mean velocity of the left half (agar) was $5.9 \mathrm{~m} / \mathrm{s}$ and that of the right half (gelatin) was $3.7 \mathrm{~m} / \mathrm{s}$, which had good agreement with the measured $v_{S}$ of each phantom in the first experiment.

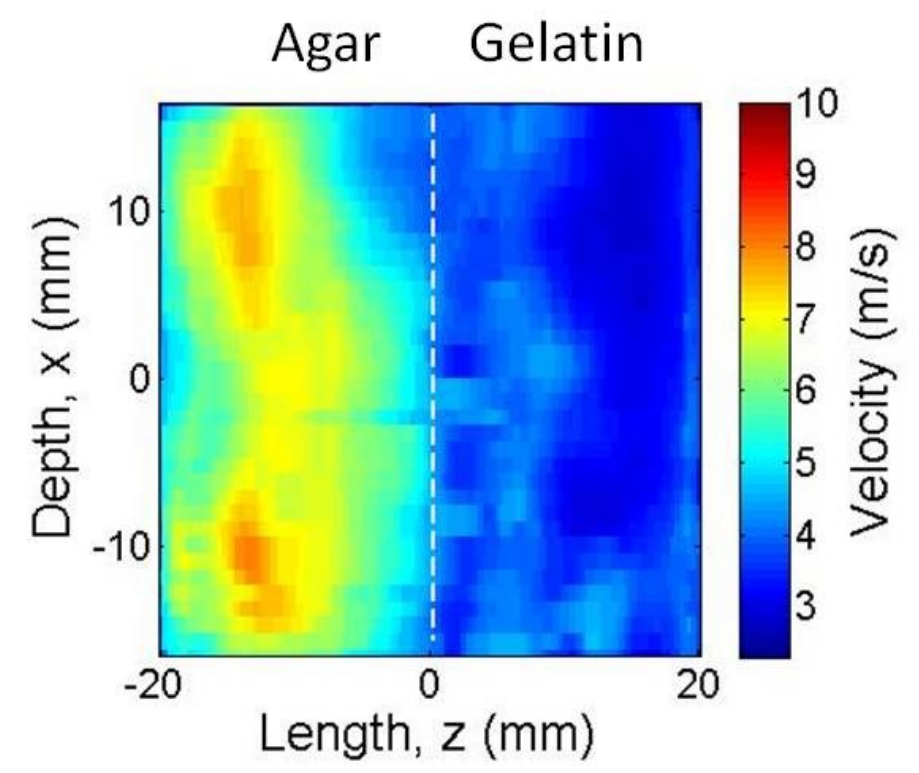

Figure 3.12. Measured SW velocity of the combined phantom.

It was noted that the variations of the measured $v_{S}$ from the average velocities could be resulted from the inhomogeneity of the fabricated phantoms and the estimation error of the phase slope of the analytic $V_{F}^{*}$ signal calculated by the Hilbert transform. The accuracy of Hilbert transform was highly dependent on the shape or frequency of processed signals [95]. 


\subsection{Summary}

The research in this chapter was aiming to develop a measurement method of SW velocity, and to address the Problem 1 described in Section 1.2 related to the undesired SW reflections. It was found that the scanning time delay resulted in an artificial spatial frequency shift between the induced and reflected SWs observed. Therefore the scanning time delay can be considered as an advantage factor and the forward SW can be retrieved from an interfered SW by using a spatial frequency low pass filter. With the proposed method, SW velocity was measured from the phase slope of the retrieved forward SW. Thesis Objective 1 in Section $\mathbf{1 . 3}$ was partially accomplished and Objective 2 was accomplished by the research of this chapter. However, a shortcoming of the proposed measurement method should also be noticed: the calculation of SW velocity required a prior knowledge of the relationship between the B-mode scanning speed and SW velocity, which in practice may be unknown without experiments, making the appropriate calculation of SW velocity uncertain. An improved measurement method of SW velocity would be proposed in the following Chapter 4. 


\title{
Chapter 4
}

\section{Modification of the Shear Wave Velocity Measurement}

\author{
Method
}

\subsection{Introduction}

In Chapter 3, a measurement method of SW velocity was developed by using B-mode scan of a focused ultrasound and a single external SW source. The developed method took the B-mode scanning time delay into account and also removed the undesired SW reflections. Although the method allowed higher values of SW frequency, it also required a prior knowledge of the relationship between the SW velocity and the B-mode scanning speed, i.e. which one was greater. However, such a relationship for an arbitrary specimen may not be available prior to the experiments. In this chapter, a modified SW velocity measurement method was proposed in order to address the above-mentioned shortcoming.

\subsection{Methodology}

\subsubsection{Modification of Measurement Model}

According to the measurement model in Fig. 3.2, the induced SW was the $V_{F}$ upon that configuration and it was used to calculate the $v_{S}$ due to its better signal-to-noise ratio 
(SNR). From Eq. (3.2) it can be seen that the sign of the spatial frequency of the $V_{F}, k_{F}$ $\left(=\omega_{S}\left(\frac{1}{v_{S}}-\frac{1}{v_{\text {scan }}}\right)\right)$, could be reversed if $v_{S}>v_{\text {scan }}$. Furthermore, no SW propagation would be observed if $v_{S}=v_{\text {scan }}$ since it resulted in the same magnitude values at all positions on $z$. However, the relationship between $v_{S}$ and $v_{\text {scan }}$ may be unknown for an arbitrary specimen. As a contrast, $V_{B}$ never changed the sign of its spatial frequency, $k_{B}$ $\left(=\omega_{S}\left(\frac{1}{v_{S}}+\frac{1}{v_{s c a n}}\right)\right)$, according to Eq. (3.3), regardless of the relationship between $v_{S}$ and $v_{\text {scan }}$. Therefore, the measurement model in this chapter was modified to make the induced $\mathrm{SW}$ become $V_{B}$ so that the prior knowledge of the relationship between $v_{S}$ and $v_{\text {scan }}$ would no longer be required.

In order to realize such desire, the modified measurement model was shown in Fig. 4.1. The origin of the coordinate system was at the center of the ultrasound probe and SW source. The ultrasound probe was placed on the top of the specimen. The SW source was now contacting the other side of the specimen from the location it used to be in Fig. 3.2 so that it induced the SW against the direction of B-mode scanning (toward the negative $z$-direction). The induced SW may be reflected at the other side or the boundaries in the specimen. Tissue displacements along the $x$-direction caused by the SWs were detected by the ultrasound probe. The SW that had the same propagation direction (positive $z$-direction) as the B-mode scanning direction was termed as forward $\mathrm{SW}\left(V_{F}\right)$ and that with opposite direction (negative $z$-direction) was termed as backward $\mathrm{SW}\left(V_{B}\right)$. Therefore, the mathematical expressions of $V_{F}$ and $V_{B}$ remained the same as Eqs. (3.2) and (3.3), respectively. Only the induced SW became the $V_{B}$ in this chapter. 


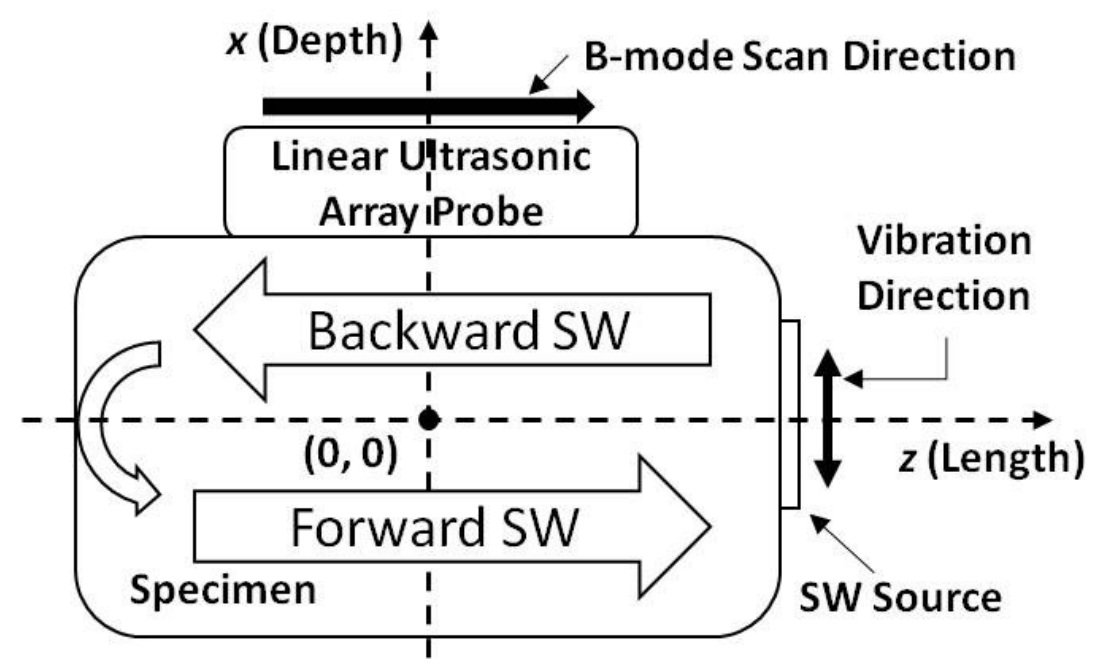

Figure 4.1. Modified measurement model of SW velocity.

At a certain time $t$, the temporal term $\omega_{S} t$ in Eqs. (3.1), (3.2) and (3.3) became constant, so these equations were simplified to:

$$
\begin{aligned}
& V(z)=V_{F}(z)+V_{B}(z) \\
& V_{F}(z)=A_{F} \cos \left[-\omega_{S}\left(\frac{1}{v_{S}}-\frac{1}{v_{\text {scan }}}\right) z+\varphi_{F}\right] \\
& V_{B}(z)=A_{B} \cos \left[\omega_{S}\left(\frac{1}{v_{S}}+\frac{1}{v_{\text {scan }}}\right) z+\varphi_{B}\right]
\end{aligned}
$$

In order to estimate $v_{S}, V_{B}$ must be isolated from the $V$. Since the $k_{F}$ and $k_{B}$ were different, $V_{B}$ can be retrieved from the obtained $V$ by using a spatial frequency filter. A spatial bandpass filter $\left(\boldsymbol{B P} \boldsymbol{F}_{\text {spatial }}\right)$, which only passed $V_{B}$, was designed and applied on the interfered SW $V$. A window function $\boldsymbol{W I N D O W}(z)$ was then multiplied on the filtered $V_{B}$ in order to reduce the spectral leakage effect due to the waveform discontinuity. Together, these two steps can be expressed by Eq. (4.4):

$$
V_{B}(z)=\boldsymbol{B P F}_{\text {spatial }}\{V(z)\} \cdot \boldsymbol{W I N D O W}(z) .
$$


Then after the Hilbert transform $\left(\mathcal{H}_{\mathrm{z}}\right)$ on the filtered $V_{B}(z)$, the $v_{S}$ at each $z$ would finally be calculated through the following equations:

$$
\begin{aligned}
& V_{B}^{*}(z)=V_{B}(z)+j \cdot \mathcal{H}_{z}\left\{V_{B}(z)\right\}, \\
& \begin{aligned}
\angle V_{B}^{*}(z) & =\tan ^{-1}\left[\frac{\operatorname{Im}\left[V_{B}{ }^{*}(z)\right]}{\operatorname{Re}\left[V_{B}{ }^{*}(z)\right]}\right] \\
& =\omega_{S}\left(\frac{1}{v_{S}}+\frac{1}{v_{\text {scan }}}\right) z+\varphi_{B},
\end{aligned} \\
& v_{S}(z)=\frac{1}{\frac{1}{\omega_{S}} \cdot \frac{\partial\left[\angle V_{B}{ }^{*}(z)\right]}{\partial z}-\frac{1}{v_{\text {scan }}}} .
\end{aligned}
$$

\subsubsection{Removal of Shear Wave Reflection}

In order to remove the $V_{B}$ from $V$ using a $\boldsymbol{B P F}_{\text {spatial, }}$, the spatial frequencies of $V_{F}$ and $V_{B}$, $k_{F}$ and $k_{B}$, respectively, must be separable within the passband of the $\boldsymbol{B P F}$ spatial. Therefore, no overlap should be existed between $k_{F}$ and $k_{B}$ within the passband. The $k_{F}$ and $k_{B}$ were normalized by $\omega_{S}$ as $\xi_{F}$, and $\xi_{B}$, respectively, by Eqs. (4.8) and (4.9):

$$
\begin{aligned}
& \xi_{F}=\frac{k_{F}}{\omega_{S}}=\left|\frac{1}{v_{S}}-\frac{1}{v_{\text {scan }}}\right|, \\
& \xi_{B}=\frac{k_{B}}{\omega_{S}}=\frac{1}{v_{S}}+\frac{1}{v_{\text {scan }}} .
\end{aligned}
$$

Note the $\xi_{F}$ in Eq. (4.8) was an absolute value since we did not consider the artificial movement direction of $V_{F}$ in the spectrum analysis. Then, the variations of $\xi_{F}$ and $\xi_{B}$, respectively, with respect to the $v_{S}$ under our B-mode scanning condition of $v_{\text {scan }}$ $=2.26 \mathrm{~m} / \mathrm{s}$, were calculated and plotted in Fig.4.2. 


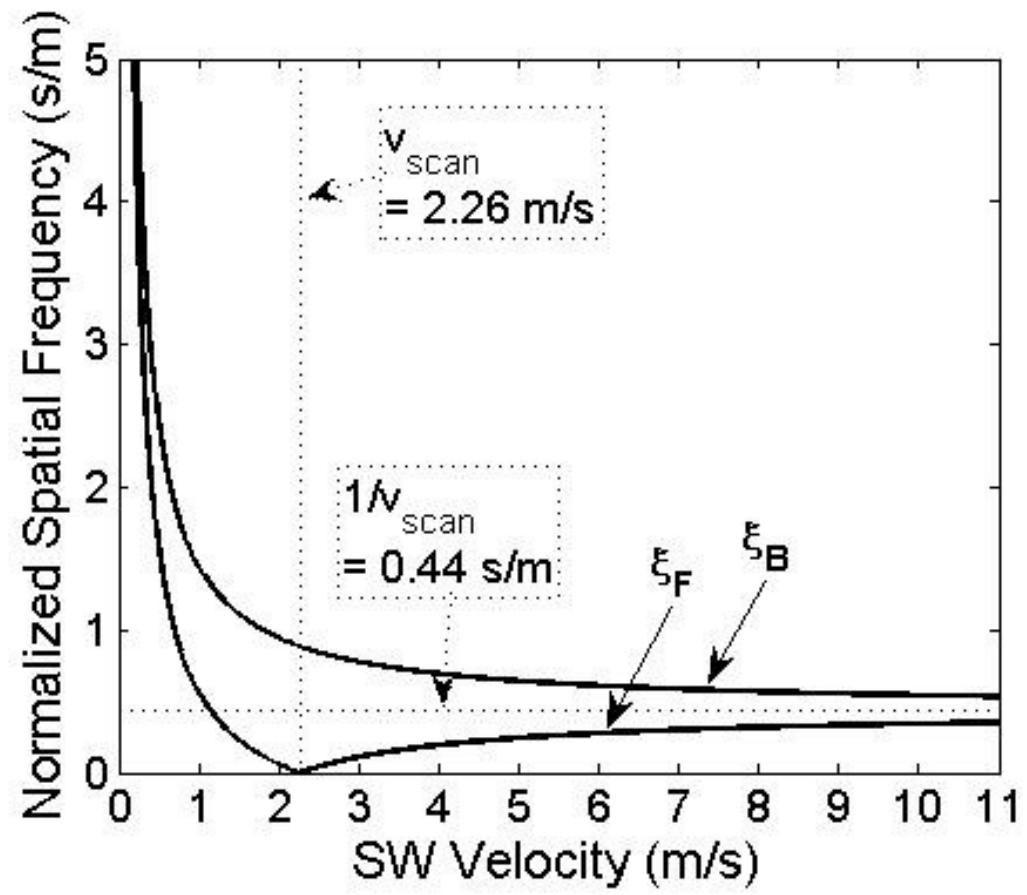

Figure 4.2. Normalized spatial frequencies of forward and backward SWs with respect to the $\mathrm{SW}$ velocity when $v_{\text {scan }}=2.26 \mathrm{~m} / \mathrm{s}$.

It can be seen that both $\xi_{F}$ and $\xi_{B}$ decreased from an infinite large with respect to the $v_{S}$ when $v_{S}<v_{\text {scan }}$. Then $\xi_{F}$ reached its minimum value zero at $v_{S}=v_{\text {scan }}$. When $v_{S}>$ $v_{\text {scan }}$, the $\xi_{B}$ continued the downtrend while the $\xi_{F}$ started increasing with respect to the $v_{S}$. In addition, $\xi_{F}$ and $\xi_{B}$ were symmetric to $1 / v_{\text {scan }}(0.44 \mathrm{~s} / \mathrm{m})$ when $v_{S}>v_{\text {scan }}$. According to Fig. 4.2, in order to separate $\xi_{F}$ and $\xi_{B}$, Eq. (4.10) should be satisfied:

$$
\min \left\{\xi_{B}\right\} \geq \max \left\{\xi_{F}\right\}
$$

When $v_{\text {scan }}=2.26 \mathrm{~m} / \mathrm{s}$, this condition was satisfied in the $v_{S}$ range of $1.02 \mathrm{~m} / \mathrm{s} \leq v_{S}$ $\leq 10 \mathrm{~m} / \mathrm{s}$, as illustrated in Fig. 4.3(a). Thus the $\boldsymbol{B P F}_{\text {spatial }}$ could be designed to retrieve $\xi_{B}$ accordingly, as illustrated in Fig. 4.3(b). The lower and higher spatial cut-off frequencies were calculated $0.54 \mathrm{~s} / \mathrm{m}$ for $v_{S}=10 \mathrm{~m} / \mathrm{s}$, and $1.42 \mathrm{~s} / \mathrm{m}$ for $v_{S}=1.02 \mathrm{~m} / \mathrm{s}$, respectively, by using Eq. (4.9). 

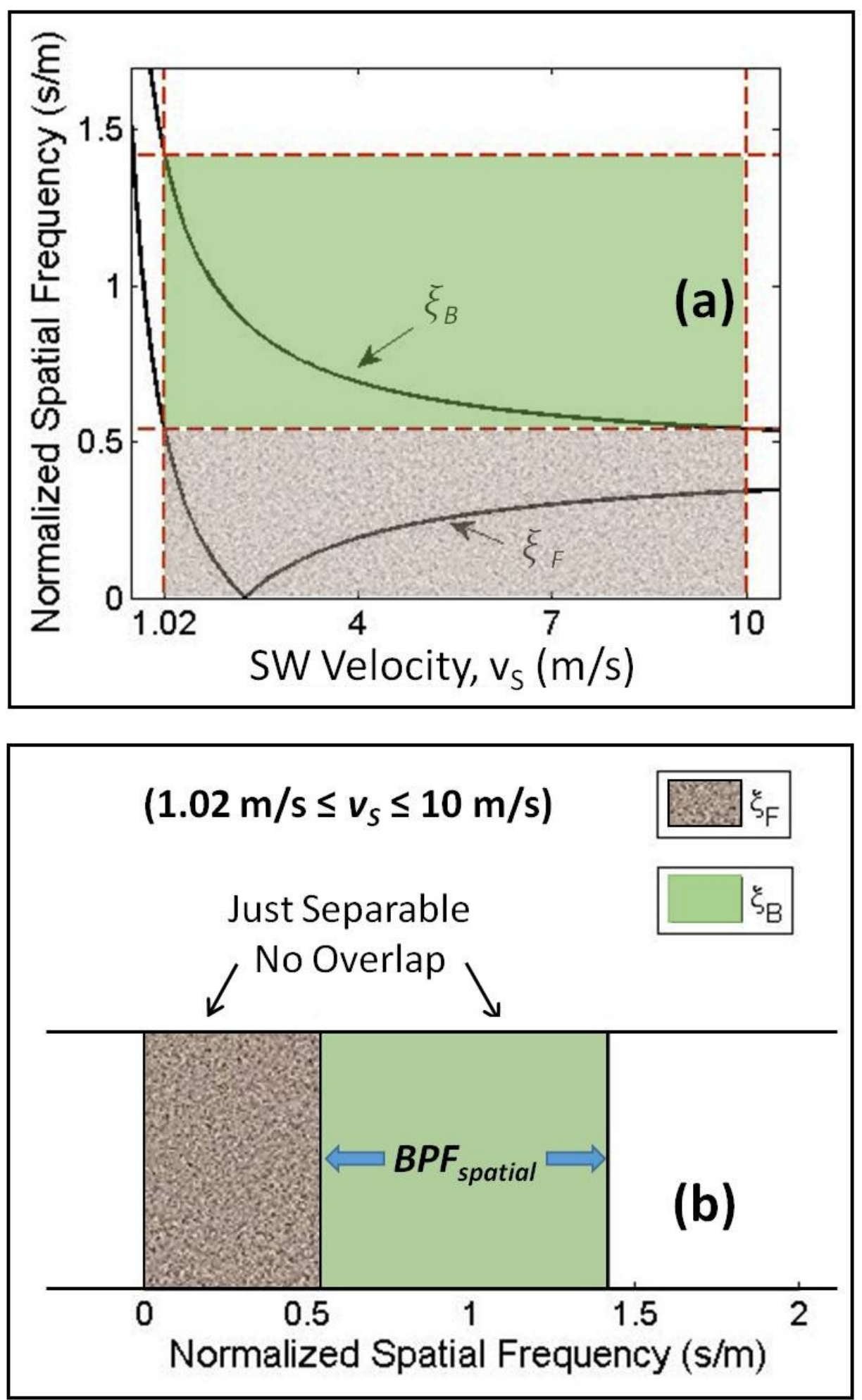

Figure 4.3. (a) The dispersion of normalized spatial frequencies of forward and backward SWs. (b) The coverages of normalized spatial frequencies of forward and backward SWs when $1.02 \mathrm{~m} / \mathrm{s} \leq v_{S} \leq 10 \mathrm{~m} / \mathrm{s}$. $v_{\text {scan }}=2.26 \mathrm{~m} / \mathrm{s}$. 
It should be noted that for most of the soft tissues, their $v_{S}$ was in the range from 1 $\mathrm{m} / \mathrm{s}$ to $10 \mathrm{~m} / \mathrm{s}$ [86]. Since our ultrasound system of which $v_{\text {scan }}$ is $2.26 \mathrm{~m} / \mathrm{s}$ was capable of measuring $v_{S}$ from $1.02 \mathrm{~m} / \mathrm{s}$ to $10 \mathrm{~m} / \mathrm{s}$, the proposed measurement method of SW velocity was applicable to characterize most of soft tissue materials.

\subsubsection{Selection of Shear Wave Frequency}

In order to observe the waveform of $V_{B}$ along $z$-direction without aliasing, the spatial sampling interval given by $\Delta z$ needs to satisfy the Nyquist sampling theorem expressed by Eq. (4.11). Therefore the highest applicable $f_{S}$ was limited and expressed by Eq. (4.12).

$$
\begin{aligned}
& \Delta z<\frac{1}{2} \cdot \frac{2 \pi}{k_{B}} \\
& f_{S}<\frac{1}{2 \cdot \Delta z\left(\frac{1}{v_{S}}+\frac{1}{v_{\text {scan }}}\right)} .
\end{aligned}
$$

In addition, in order to accurately determine the spatial frequency of $V_{B}$, it was desirable that the FOV in $z$-direction $\left(\mathrm{FOV}_{\mathrm{z}}\right)$ contained at least one full period/wavelength $\left(\lambda_{B}\right)$ of the measured waveform of $V_{B}$. This restriction was expressed in Eq. (4.13). Therefore the lowest applicable $f_{S}$ was limited as expressed by Eq. (4.14).

$$
\begin{aligned}
& \lambda_{F} \leq F O V_{Z}, \quad \text { where } \lambda_{B}=\frac{2 \pi}{k_{B}}, \\
& f_{S} \geq \frac{1}{\operatorname{FOV}_{z} \cdot\left(\frac{1}{v_{S}}+\frac{1}{v_{\text {Scan }}}\right)} .
\end{aligned}
$$

For $v_{S}$ ranging from $1.02 \mathrm{~m} / \mathrm{s}$ to $10 \mathrm{~m} / \mathrm{s}$, according to our measurement conditions of $\Delta z=315 \mu \mathrm{m}, \mathrm{FOV}_{\mathrm{z}}=40 \mathrm{~mm}$, and $v_{\text {scan }}=2.26 \mathrm{~m} / \mathrm{s}$, the restriction of the $f_{S}$ with respect 
to the $v_{S}$ was calculated and plotted in Fig. 4.4. The upper limits of $f_{S}$ calculated by Eq. (4.12) were indicated by dash-line and the lower limits calculated by Eq. (4.14) were indicated by solid-line for each $v_{S}$. Therefore, the highest $f_{S}$ should be less than $1115.6 \mathrm{~Hz}$ when $v_{S}=1.02 \mathrm{~m} / \mathrm{s}$ in Eq. (4.12), and the lowest applicable $f_{S}$ was $46.1 \mathrm{~Hz}$ (inclusive) when $v_{S}=10 \mathrm{~m} / \mathrm{s}$ in Eq. (4.14). As discussed in Chapter 3, the highest $f_{S}$ that was obtained by using $V_{F}$ is $2847 \mathrm{~Hz}$ (refer to Fig. 3.4). Although the highest $f_{S}$ obtained for $V_{F}$ was about 2.6 times greater than that obtained for $V_{B}$, the preferred $f_{S}$ range for a SW measurement for soft tissue characterization was typically from $100 \mathrm{~Hz}$ to $1000 \mathrm{~Hz}$ due to the significant attenuation of SW at higher frequencies. Thus the applicable range of $f_{S}$ with $V_{B}$ in this modified measurement model was still wide enough for $v_{S}$ measurements for the soft tissue characterization.

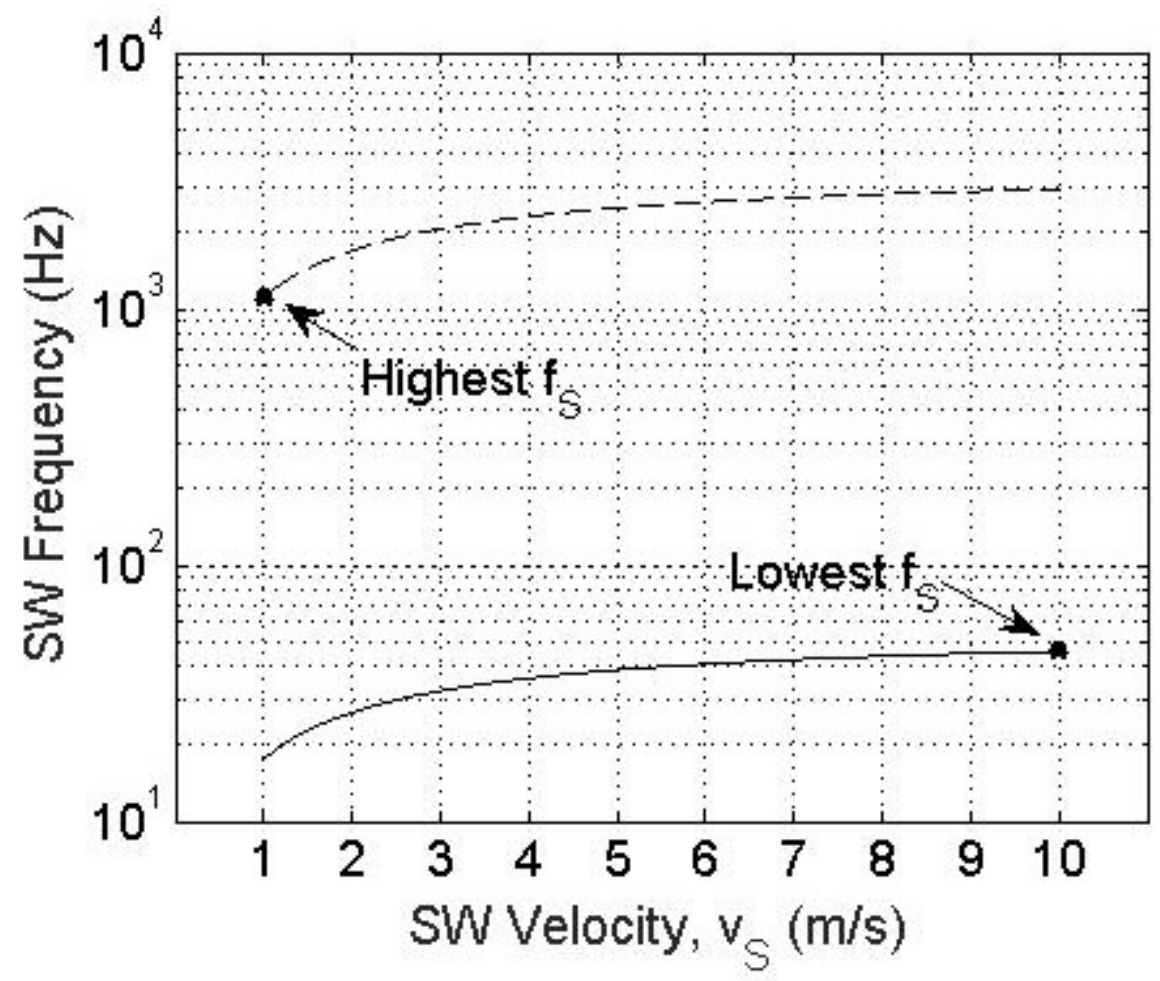

Figure 4.4. The highest and lowest applicable SW frequency with respect to shear wave velocity. 


\subsection{Experiment}

\subsubsection{Experimental Configuration}

A soft tissue mimicking phantom was fabricated for the experiments to verify the modified $v_{S}$ measurement model and method. Fig. $\mathbf{4 . 5}$ presents a structure of the phantom, its photograph of top surface at the $x-z$ plane, and its B-mode ultrasound image at $y=0$. The phantom was composed with a relatively soft surrounding body (as a background area) and a relatively stiffer inclusion. The background was made by $5-\mathrm{W} \%$ gelatin in water and shaped to a cube with a side length of $50 \mathrm{~mm}$. The stiffer inclusion was made by $2-\mathrm{W} \%$ agar in water and shaped to a cylinder with a diameter of $20 \mathrm{~mm}$ and the length of $50 \mathrm{~mm} .2-\mathrm{V} \%$ isopropanol (70\% purity) was added to both parts to prevent bacteria growth. The graphite particles with a nominal diameter of $50 \mu \mathrm{m}$ were dispersed in each part as ultrasound scatterers. The tactile feeling of the inclusion part was stiffer than that of the background part. The inclusion part was first made and shaped. It stood on the center of a cubic mould along the $y$-direction. Then, the dissolved gelatin solution for the surrounding part was poured into the cubic mould around the cylindrical inclusion part. The phantom was kept in a fridge for more than 24 hours to solidify.

The experimental configuration was depicted in Fig. 4.6. A thin Plexiglas plate with a size of 10-mm square was contacted onto the side surface of the phantom as a SW vibration source. The square plate was connected to an acoustic speaker through an Lshaped rigid plastic rod, and the speaker was driven continuously at $230 \mathrm{~Hz}$ during the experiment. The ultrasonic probe was attached on the top surface of the phantom. The 
SW was induced toward the negative $z$-direction and the B-mode scanning direction was toward the positive $z$-direction as shown in Fig. 4.1.
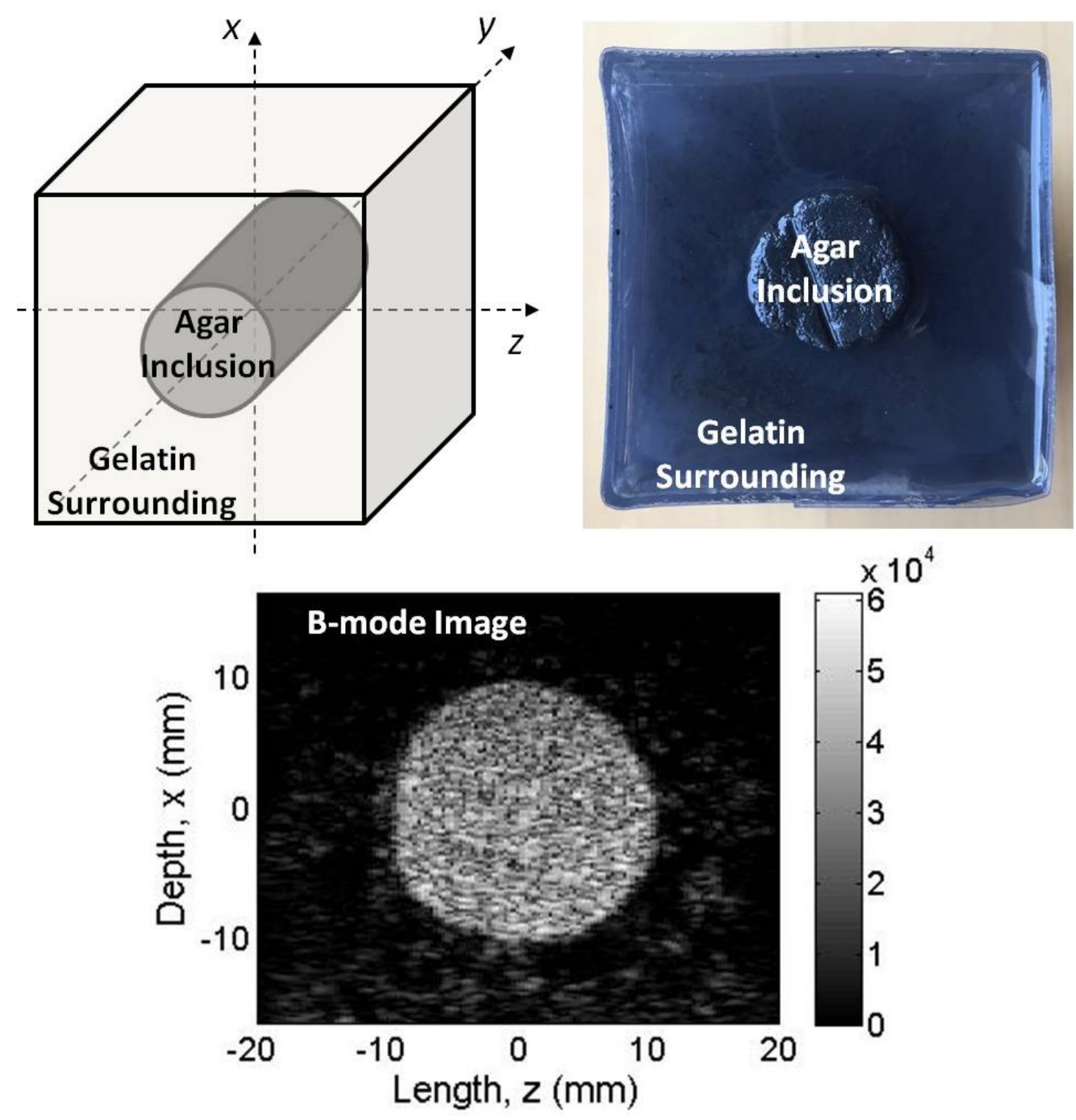

Figure 4.5. A structure (top left), photograph (top right), and B-mode ultrasound image (bottom) of the phantom fabricated. 


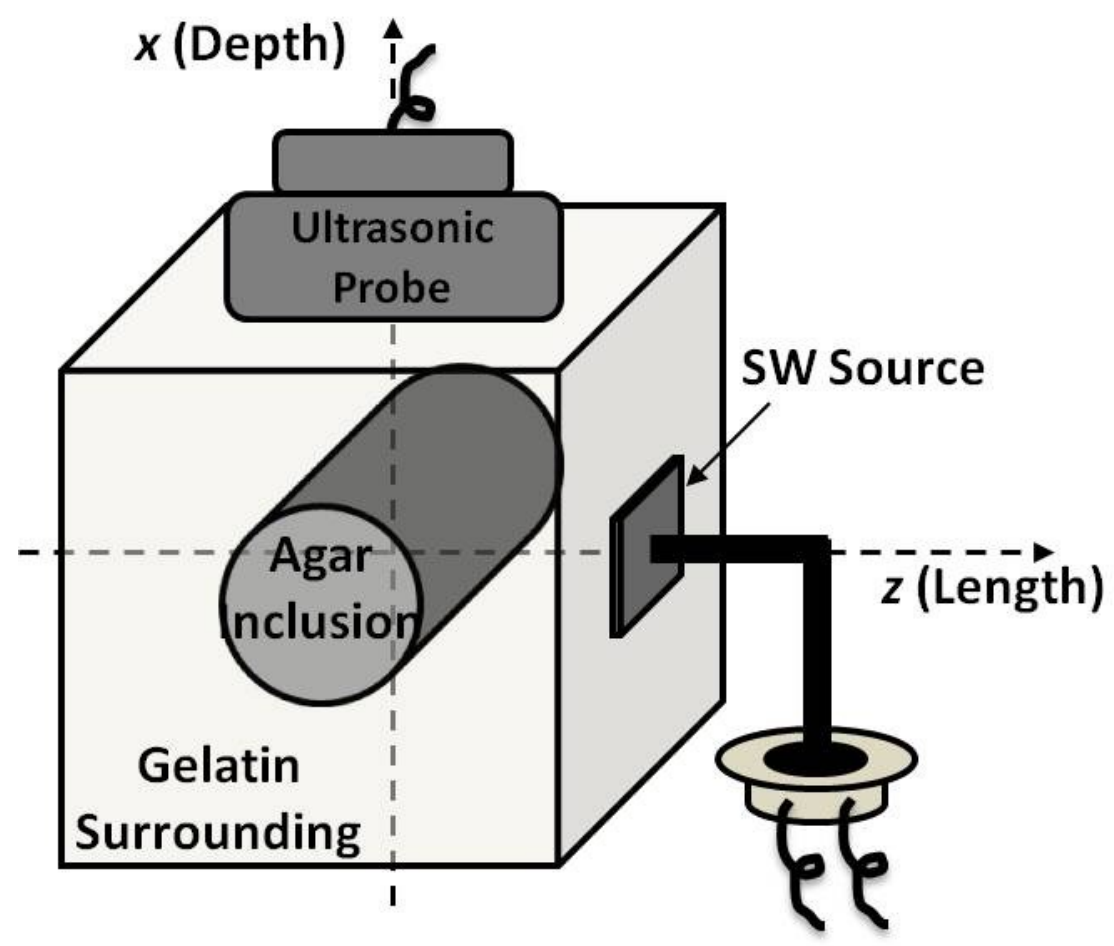

Figure 4.6. Experimental configuration.

\subsubsection{Experimental Result}

The filtered $V_{B}$ obtained at a specific B-mode frame was shown in Fig. 4.7(a). The waveform profile of the $V_{B}$ at the centerline along the $z$-direction (indicated by the dashline in Fig. 4.7 (a)) was also shown in Fig. 4.7(b). It can be seen that the SW wavelengths of the inclusion area (from $z=-10 \mathrm{~mm}$ to $z=10 \mathrm{~mm}$ at $x=0$ ) were greater than those of the background area. Figure 4.7(c) represents the spatial frequency spectrum of the filtered $V_{B}$ in Fig. 4.7(b). The $\boldsymbol{B P} \boldsymbol{F}_{\text {spatial }}$ in Eq. (4.4) was designed accordingly as described in Section 4.2.2, which covered a $v_{S}$ range from $1.02 \mathrm{~m} / \mathrm{s}$ to 10 $\mathrm{m} / \mathrm{s}$. 

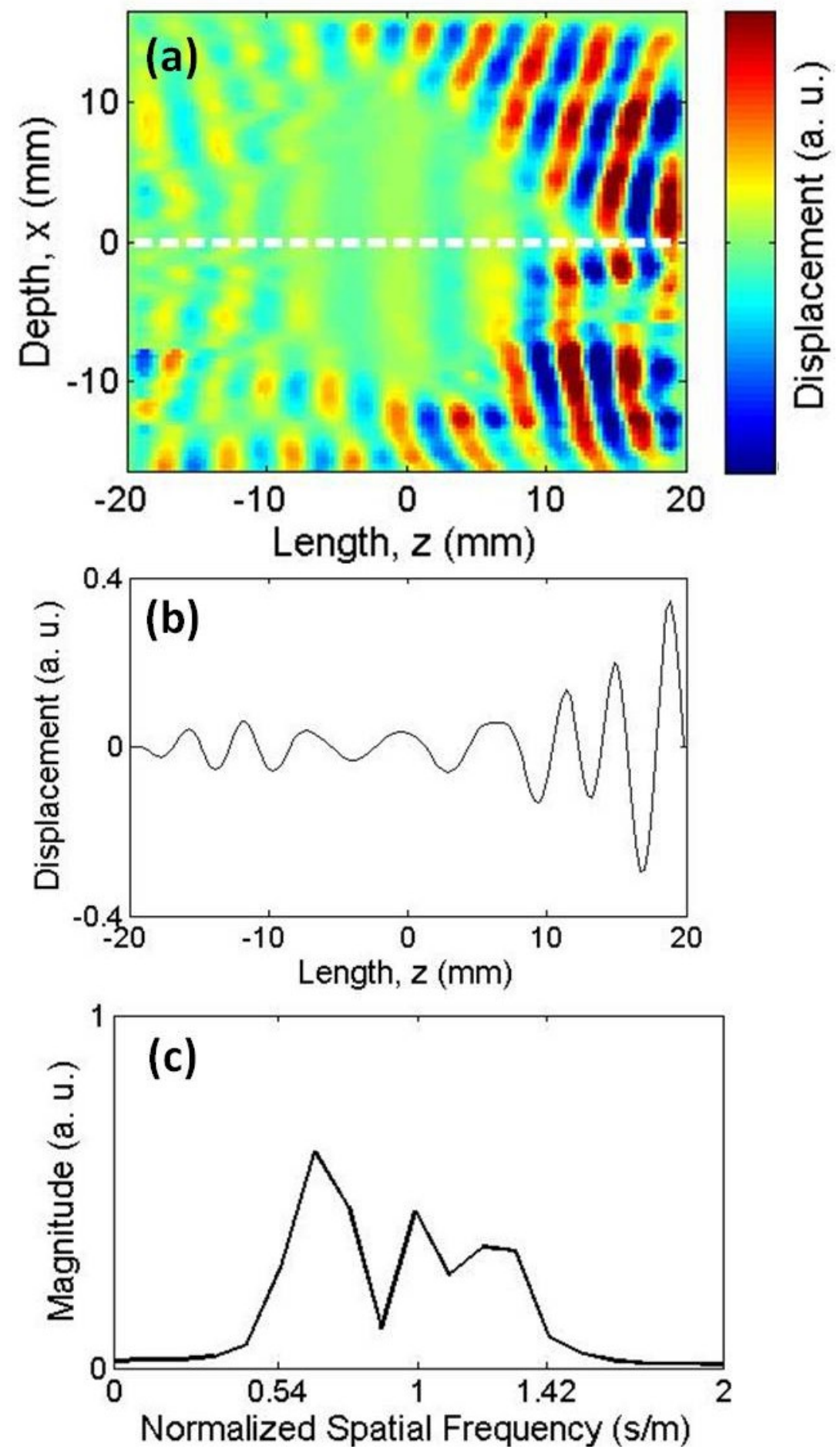

Figure 4.7. Measurement results of (a) filtered backward SW $\left(V_{B}\right)$ in the phantom, (b) profile of SW waveform on the centerline as indicated in (a), and (c) the normalized spatial frequency spectrum of filtered backward $\mathrm{SW}\left(V_{B}\right)$ at centerline. 
The $v_{S}$ of the phantom was calculated by using Eq. (4.7). The result of $v_{S}$ image was averaged with 180 frames. The $v_{S}$ map was shown in Fig. 4.8(a). Any $v_{S}$ value calculated outside the range of $1.02 \mathrm{~m} / \mathrm{s}$ to $10 \mathrm{~m} / \mathrm{s}$ would be considered invalid and blackened out with N/A notation in Fig. 4.8(a). A median filter was applied on the $v_{S}$ image to remove outlier results. The mean value of $v_{S}$ of the inclusion area was calculated inside the ROI as indicated by the 10-mm radius dash circle in Fig. 4.8(a). The N/A values were excluded from the calculations. The mean value of the $v_{S}$ in the inclusion area was $4.4 \mathrm{~m} / \mathrm{s}$ and the STD was $1.7 \mathrm{~m} / \mathrm{s}$, and the values of the background (the area outside the dash circle) were $1.9 \mathrm{~m} / \mathrm{s}$ and $0.36 \mathrm{~m} / \mathrm{s}$, respectively. The $v_{S}$ image was superimposed onto the B-mode image in Fig. 4.8(b). The $v_{S}$ image contrast between two areas had a good agreement with its physical structure as seen in the B-mode image. The $v_{S}$ of the relatively stiffer inclusion was 2.3 times greater than that of the relatively softer background. 

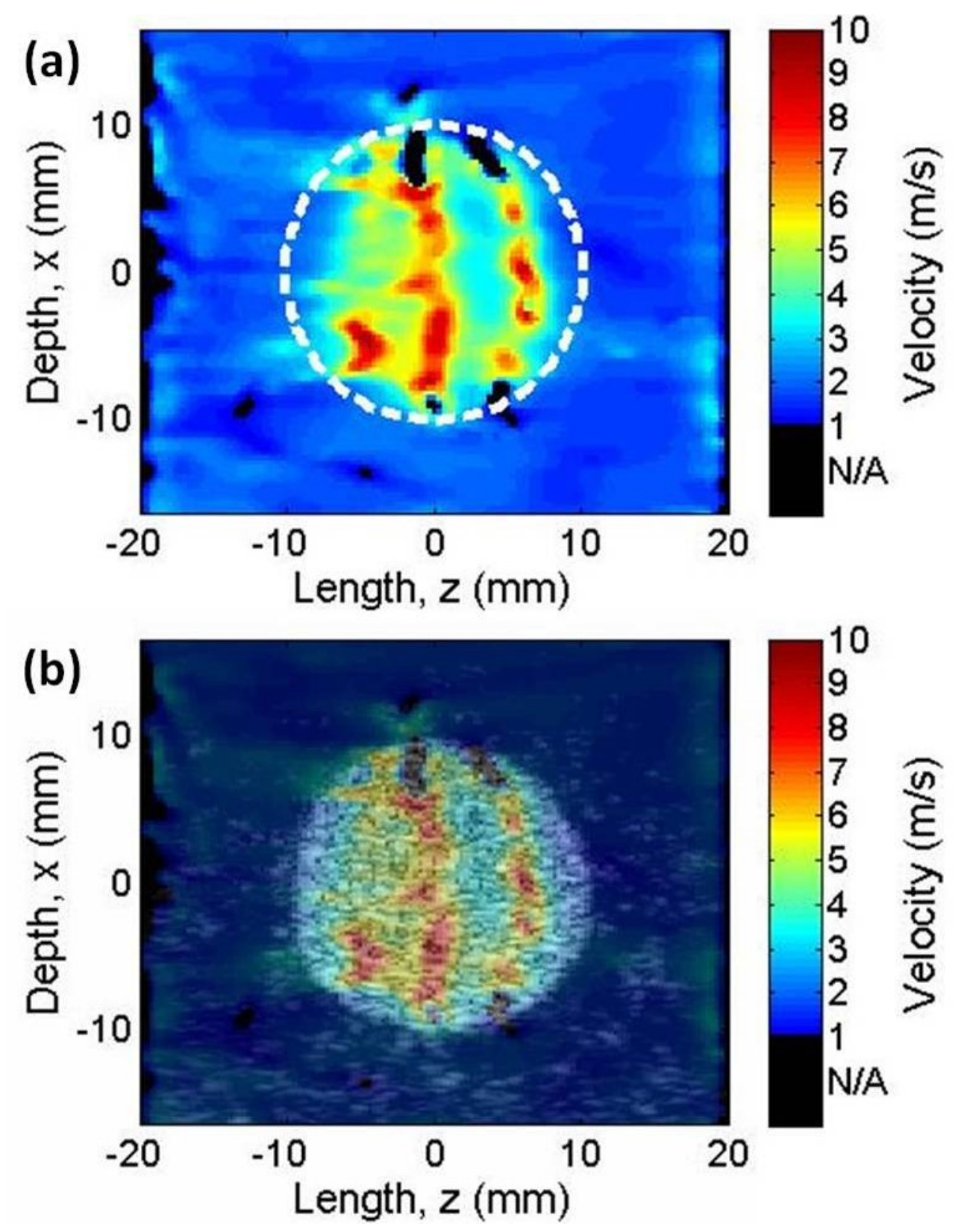

Figure 4.8. (a) Measurement result of SW velocity, dash circle indicated the area of the inclusion used to determine the mean value of the SW velocity. (b) A superposition of Bmode image and $\mathrm{SW}$ velocity. 


\subsection{Discussions}

\subsubsection{Numerical Validation of Phantom Experiment}

It was seen that the values of $v_{S}$ were greater around $z=0$ and $\pm 5 \mathrm{~mm}$ than the $v_{S}$ mean value of the inclusion. This could result probably from a calculation error in the Hilbert transform. Validation was conducted by using numerical simulation. The simulation mimicked the phantom experiment as presented in Section 4.3.1, and constructed numerically a stiffer $10 \mathrm{~mm}$-radius circular inclusion with softer background, with SW induced toward negative $z$-direction. The $v_{S}$ of inclusion was assumed $5.2 \mathrm{~m} / \mathrm{s}$ and that of the background was $1.8 \mathrm{~m} / \mathrm{s}$. These $v_{S}$ values were used to simulate $V_{F}$ and $V_{B}$ waveforms using Eqs. (4.2) and (4.3), respectively. These $v_{S}$ values were used in the simulation since they would yield similar calculation results as obtained from the experiment (see below). The $f_{S}, v_{\text {scan }}, \mathrm{FOV}$, and $\boldsymbol{B} \boldsymbol{P} \boldsymbol{F}_{\text {spatial }}$ were the same as those used in the experiment. The simulation results corresponding to the experimental results in Fig. 4.7 were presented in

Fig. 4.9. Then the $v_{S}$ image calculated from the simulated $V_{B}$ in Fig. 4.9(a) was given in Fig. 4.10. 

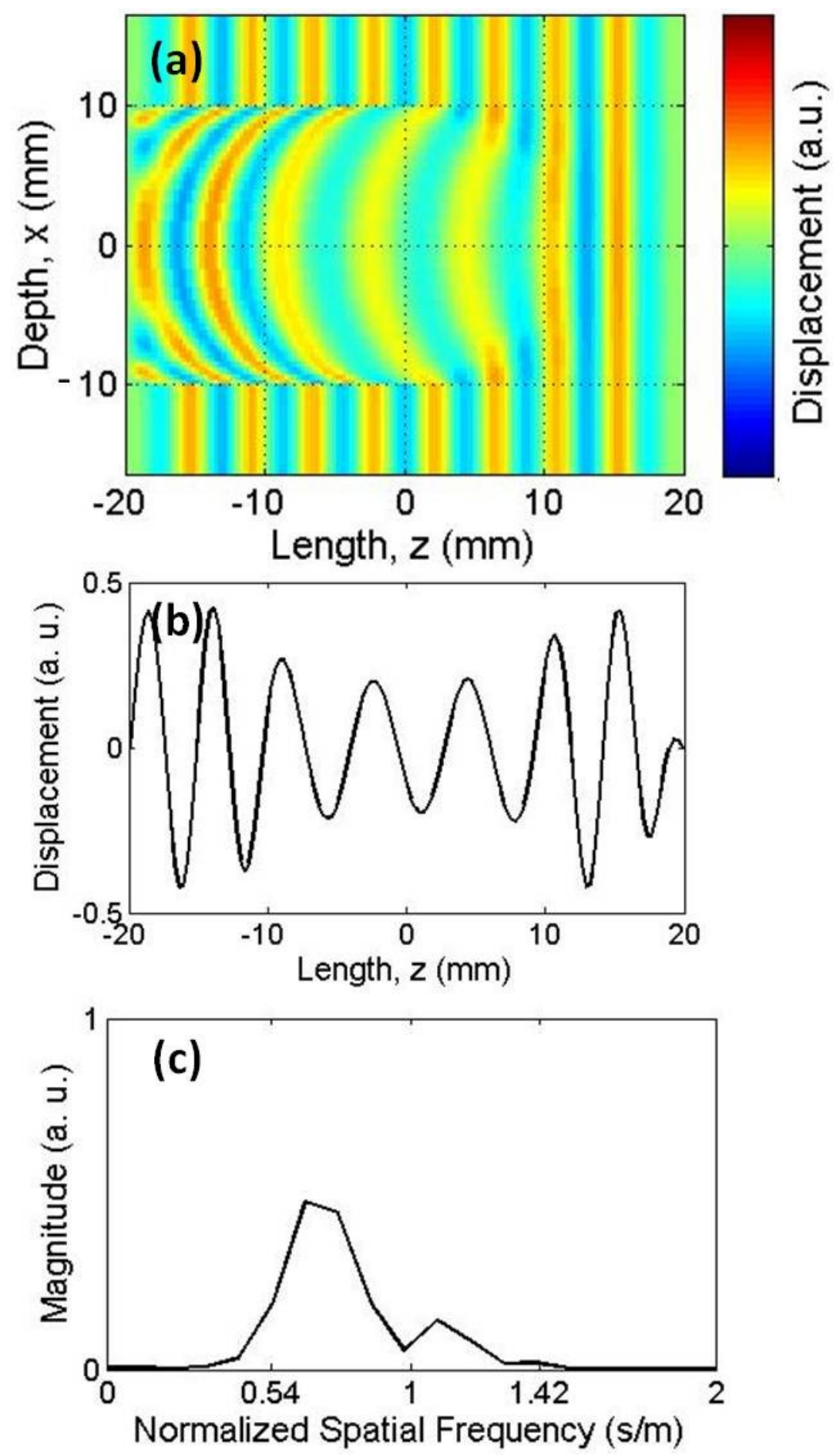

Figure 4.9. Simulation results of (a) filtered backward $\mathrm{SW}\left(V_{B}\right)$, (b) profile of SW waveform on the centerline as indicated in (a), and (c) the normalized spatial frequency spectrum of filtered backward $\mathrm{SW}\left(V_{B}\right)$ at centerline. 

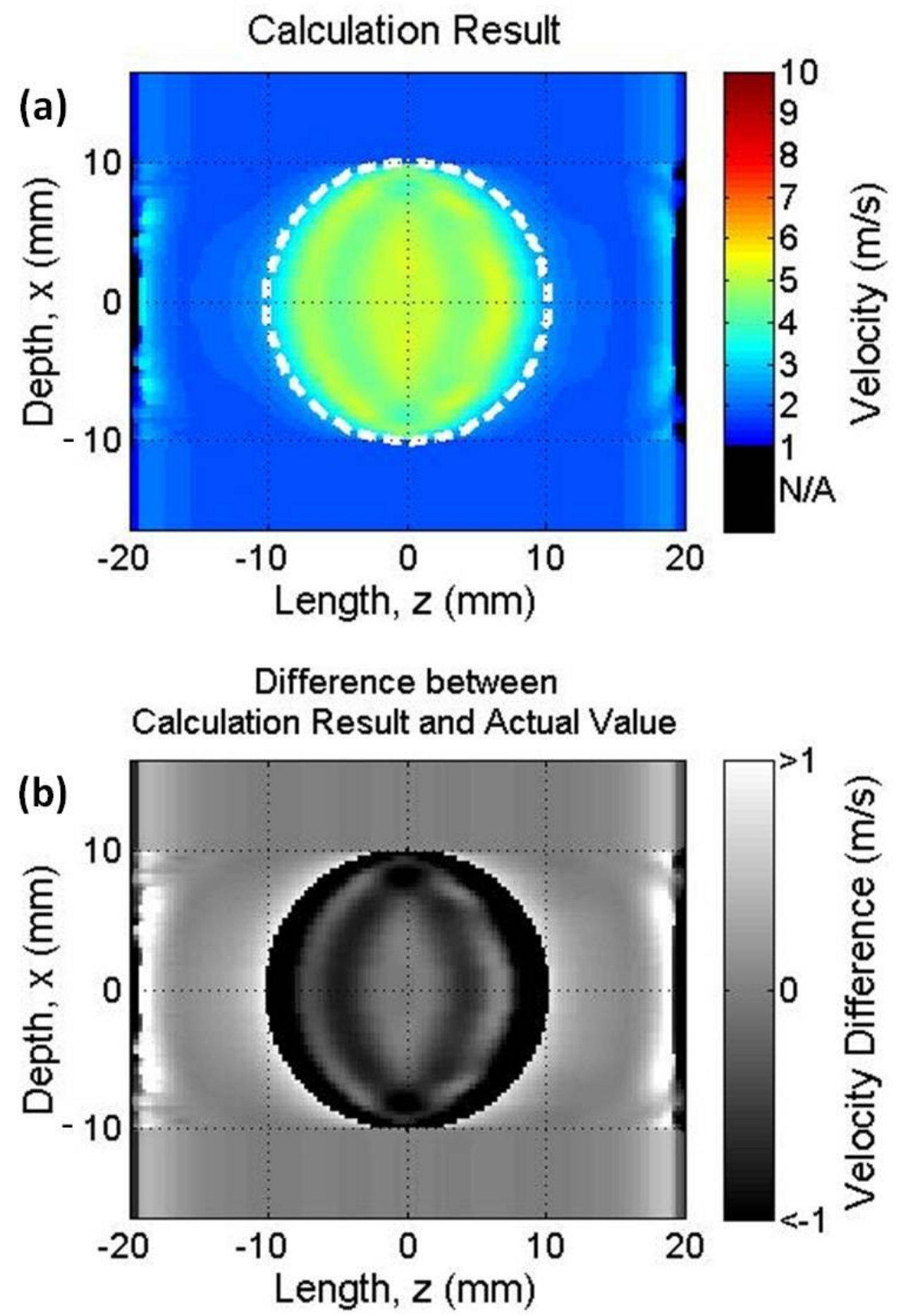

Figure 4.10. (a) SW velocity that calculated from the simulation, and (b) the difference between the calcualtion result and actual value of the SW velocity. Dash-circle indicated the area of the inclusion used to determine the mean value of the SW velocity. 
The $v_{S}$ calculated in this simulation was presented in Fig. 4.10(a). The dash-circle was the ROI that indicated the inclusion area. It was $4.4 \mathrm{~m} / \mathrm{s} \pm 0.72 \mathrm{~m} / \mathrm{s}$ (mean value \pm STD) in the inclusion area and $2.0 \pm 0.28 \mathrm{~m} / \mathrm{s}$ in the background. The difference between the calculated $v_{S}$ and its assumed value (calculated $v_{S}-$ assumed $v_{S}$ ) was presented in Fig. 4.10(b). It can be seen that the pattern of the calculated $v_{S}$ in the inclusion area had a remarkable variation/fluctuation along $z$-direction, and great deviations were also found at the boundaries and edges in Fig. 4.10(b). This was possibly due to the calculation error from the Hilbert transform. Consequently, the mean value of the calculated $v_{S}(4.4 \mathrm{~m} / \mathrm{s})$ was $0.8 \mathrm{~m} / \mathrm{s}$ less than its assumed value $(5.2 \mathrm{~m} / \mathrm{s})$ in the inclusion area. A comparison between the experimental and simulation results were listed in Table 4.1.

Table 4.1. Comparison between experimental and simulation results.

\begin{tabular}{|c|c|c|c|c|c|c|}
\hline \multirow{3}{*}{$\begin{array}{c}\text { Phantom } \\
\text { Part }\end{array}$} & \multicolumn{3}{|c|}{ Experimental Results of $v_{S}(\mathrm{~m} / \mathrm{s})$} & \multicolumn{3}{|c|}{ Simulation Results of $v_{S}(\mathrm{~m} / \mathrm{s})$} \\
\hline & \multirow{2}{*}{$\begin{array}{c}\text { Actual } \\
\text { Value }\end{array}$} & \multicolumn{2}{|c|}{ Calculation Results } & \multirow{2}{*}{$\begin{array}{l}\text { Actual } \\
\text { Value }\end{array}$} & \multicolumn{2}{|c|}{ Calculation Results } \\
\hline & & $\begin{array}{l}\text { Mean } \\
\text { Value }\end{array}$ & STD & & $\begin{array}{l}\text { Mean } \\
\text { Value }\end{array}$ & STD \\
\hline Inclusion & N/A & 4.4 & 1.7 & 5.2 & 4.4 & 0.72 \\
\hline Background & N/A & 1.9 & 0.36 & 1.7 & 2.0 & 0.28 \\
\hline
\end{tabular}

The tendency of the variation/fluctuation in the $v_{S}$ calculated from the inclusion area in Fig. 4.10(b) generally matched with that of the experimental result in Fig. 4.8(a), yet the STD of the experimental result $(1.7 \mathrm{~m} / \mathrm{s})$ was about 2.5 times greater than that of the simulation $(0.72 \mathrm{~m} / \mathrm{s})$. This could be a result from the combination of SW absorption, diffraction effect, and refraction [19], [35], [96]. Since those factors were rather complex, they were not implemented into the simulation. In addition, the spatial resolution of the $v_{S}$ 
measurement in $z$-direction was limited by the SW wavelength. Nevertheless, the modified measurement method of $v_{S}$ could still distinguish materials with the size at centimeter-level and provide reasonable $v_{S}$ values. Comprehensive study on SW propagation with a complex geometric condition could be an interest topic for future research.

\subsubsection{Influence of Shear Wave Reflection on Shear Wave Velocity \\ Measurement}

The influence of SW reflection on the measurement of $v_{S}$ was investigated by a numerical simulation in this section. A waveform of interfered SW signals $V$ was simulated by using Eqs. (4.1), (4.2) and (4.3) with $v_{\text {scan }}=2.26 \mathrm{~m} / \mathrm{s}, v_{S}=2,4,6,8 \mathrm{~m} / \mathrm{s}$, and $f_{S}=227 \mathrm{~Hz}$. The $\mathrm{FOV}_{\mathrm{z}}$ was $40 \mathrm{~mm}$ (from $z=-20 \mathrm{~mm}$ to $+20 \mathrm{~mm}$ ). An amplitude ratio between the reflected SW $\left(V_{F}\right)$ and induced SW $\left(V_{B}\right)$, as defined by $A_{F} / A_{B}$, was changed from 0 (no reflection) to 1 ( $V_{F}$ has the same amplitude as $\left.V_{B}\right)$. Then $v_{S}$ was calculated from Eqs. (4.4)-(4.7).

In the first simulation, the $\boldsymbol{B P F}_{\text {spatial }}$ was substituted by an all-pass filter, which means the removal of the reflection had not been implemented. The mean value and STD of the estimated $v_{S}$ were calculated within a ROI from $-10 \mathrm{~mm}$ to $+10 \mathrm{~mm}$. The results with respect to the amplitude ratio were shown in Fig. 4.11. In this figure, the errors were plotted as percentage, defined by: [(estimated $v_{S}-$ actual $\left.v_{S}\right) /$ actual $\left.v_{S} \times 100 \%\right]$. The error bar indicated the +/- STD. Note that the ROI was determined to avoid the relatively greater $v_{S}$ estimation error at the edge regions due to the calculation error of the Hilbert 
transform. From the results in Fig. 4.11, the error and STD of the calculated $v_{S}$ were increased with respect to the amplitude ratio for all assigned $v_{S}$ values. In addition, the calculated $v_{S}$ went beyond twice of its actual value (more than $100 \%$ error) when the amplitude ratios were greater than 0.6 for all the simulations. Therefore, such investigation indicated that measuring the $v_{S}$ without the removal of the undesired reflection would possibly lead to great errors.

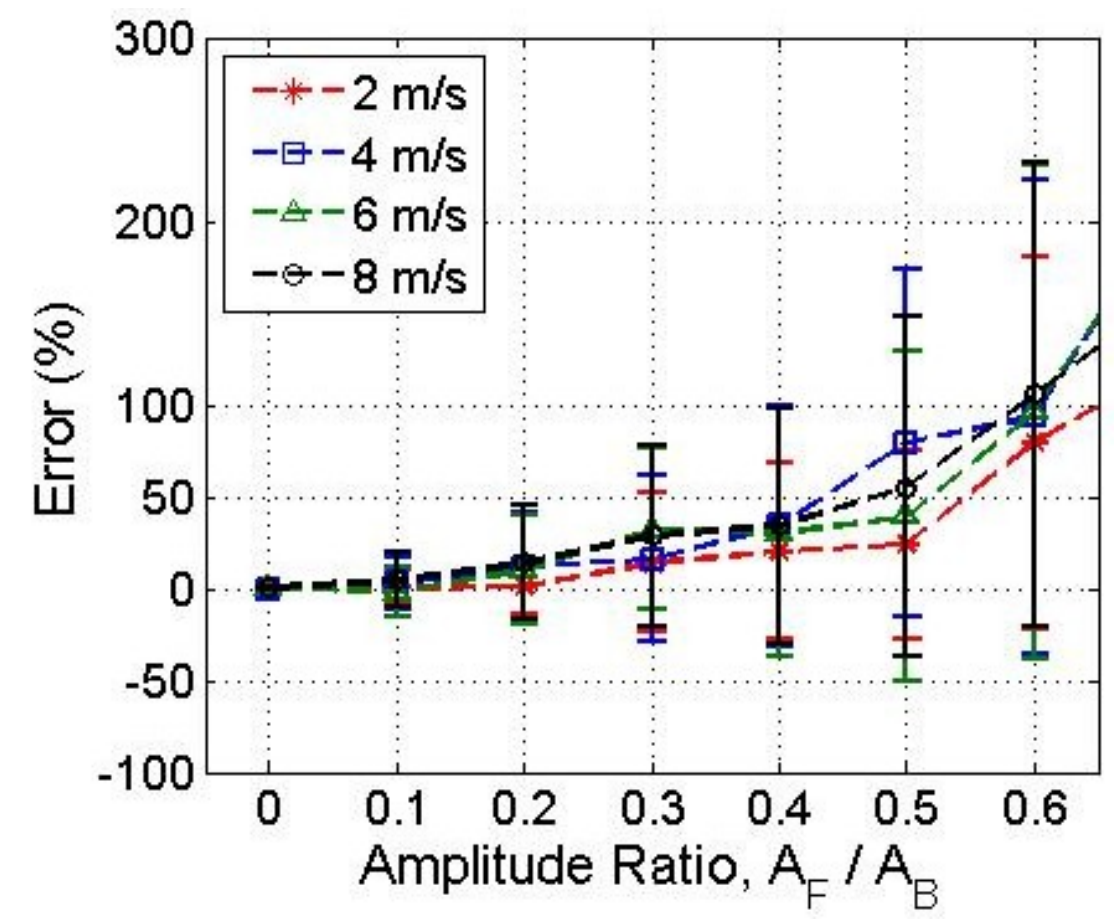

Figure 4.11. Estimation error of SW velocity without using the spatial frequency filtering. Error bar indicated the $+/$ - standard deviation.

Now in the second simulation, $\boldsymbol{B P F}_{\text {spatial }}$ was applied to investigate the effectiveness of the $\boldsymbol{B P F _ { \text { spatial } }}$ to remove the influence of SW on $v_{S}$ estimation. The $\boldsymbol{B P} \boldsymbol{F}_{\text {spatial }}$ was designed as described in Section 4.2.2. The results of estimated $v_{S}$ with

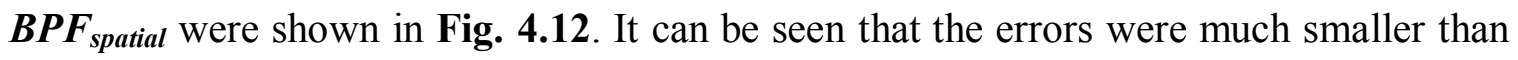
the results without $\boldsymbol{B P F}_{\text {spatial }}$ in Fig. 4.11. The mean errors were within $\pm 2 \%$ for all the 
amplitude ratio range from 0 to 1 , and the STD values were within $\pm 5 \%$. Therefore, the

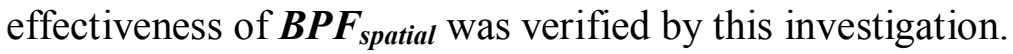

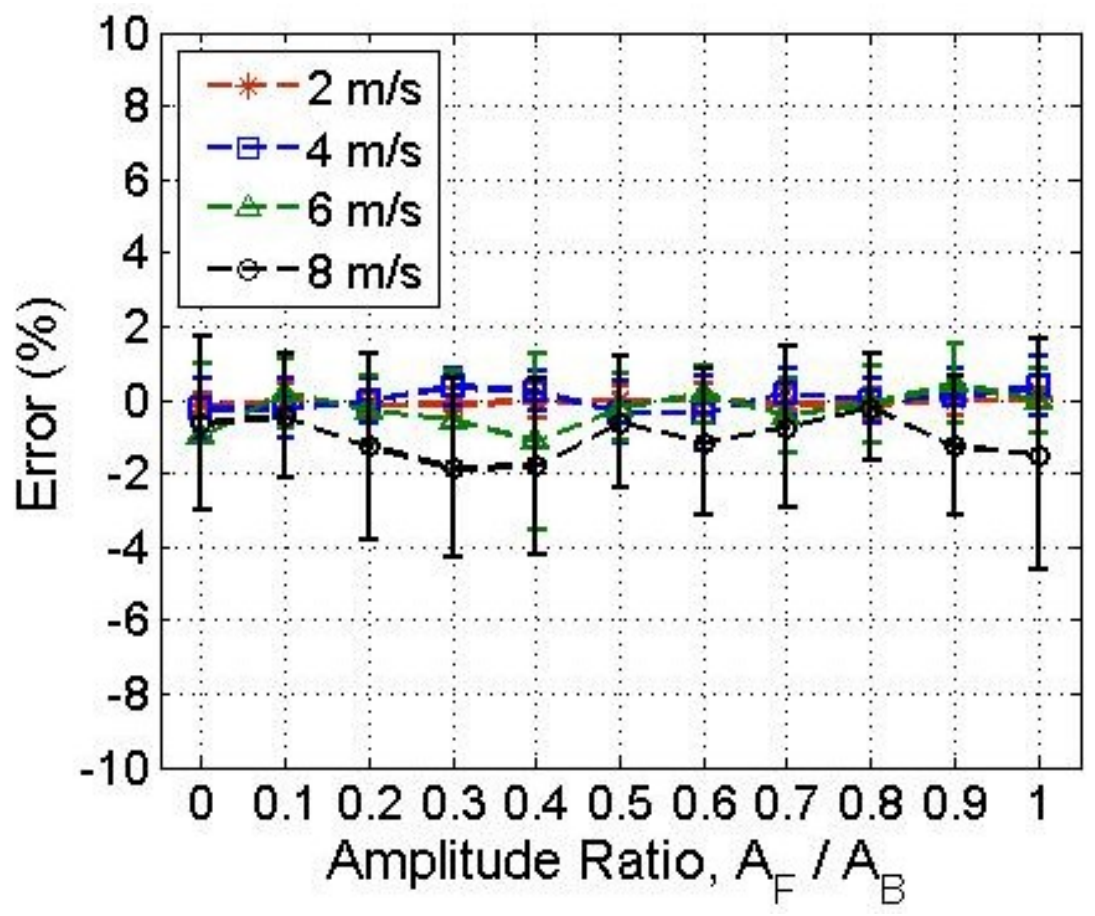

Figure 4.12. Estimation error of SW velocity with using the spatial frequency filtering. Star-dash-line represented the simulation with actual $v_{S}=2 \mathrm{~m} / \mathrm{s}$, while square-dash-line represented $4 \mathrm{~m} / \mathrm{s}$, triangle-dash-line represented $6 \mathrm{~m} / \mathrm{s}$, and circle-dash-line represented 8 $\mathrm{m} / \mathrm{s}$.

\subsection{Summary}

In this chapter, the shortcoming (prior knowledge of the relationship between the SW velocity and B-mode scanning speed) of the SW velocity measurement method proposed in Chapter 3 had been addressed. In the modified measurement method, SW was induced into a specimen opposite to the B-mode scanning direction. Phantom experiment and numerical simulations were conducted to verify the modified method. Thesis Objectives 1 in Section 1.3 had been accomplished by the research presented in this chapter. 


\section{Chapter 5}

\section{A Method to Reduce Motion Artifacts}

\subsection{Introduction}

In practical in-vivo experiments of SW measurements studied in this thesis, an ultrasound scatterer in tissues and tissue boundaries could experience motions from multiple sources, in addition to the SW propagation, such as the motions caused by the experimental environment, handheld probe movement, voluntary or involuntary muscle contraction, as well as the passive motions caused by the heart beat and respiration. Since some of the undesired motions may be unavoidable, they could influence the accurate measurement of the SW displacement.

For measurement with external SW vibration source, increasing the SW source power to induce the greater SW amplitude may be one solution since it would help to get better SNR of the SW amplitude. However, this may bring discomfort to the patient. Therefore, the smaller SW vibration would be desirable. For measurement with radiation force, amplitude of the SW was much smaller than that generated from the external source. With the radiation force technique, a motion filtering method was commonly applied to reduce the motion artifacts [97], [98]. The tissue displacement caused by the cardiac and respiratory motions could be filtered out, since their frequencies were consistent and measureable. On the other hand, the displacement due to the experimental 
random noises could be reduced by averaging the measured displacements among multiple scans [22], since those motions were considered to be non-coherent over scans. However, unexpected movement of the patient and/or probe may still exist. It was also found that if the measurement time could be shortened, the chance to have body and/or probe movements would be reduced. Therefore, the supersonic shear imaging technique [16], [22], [99] could effectively prevent the influence of any type of motion artifacts because of its ultra-short acquisition time. Nevertheless, those solutions were not applicable to our ultrasound system and proposed SW measurement method. Therefore, a method to reduce the motion artifacts was developed based on our ultrasound system and method in this chapter. Phantom experiment and numerical simulation were presented to verify the proposed method.

\subsection{Methodology}

In this study, displacement caused by the undesired motion $\left(V_{M A}\right)$ from all the sources other than $\mathrm{SW}$ displacement $(V)$ was termed as motion artifact. The tissue displacement along $x$-direction defined in Fig. 2.3, $u_{x}$ in the temporal domain was described by Eq. (5.1). It was assumed that each undesired motion had its own temporal frequency denoted by $f_{M A}$.

$$
u_{x}(t)=V(t)+V_{M A}(t) .
$$

On one hand, motion artifacts arose from experimental environment. Excitations such as an air flow from the venting system, and/or a passing-by vehicle near the building, would possibly result in resonance vibrations to the laboratory where the experiment was 
taken place. These undesired motions would be conveyed into the specimen through the measurement platform and lead to undesired tissue displacements. Practically, the $f_{M A}$ of such motion artifact can be obtained through a phantom experiment without applying SW. Since phantom did not move by itself and all the motion frequencies obtained in the phantom experiment were considered as the motion artifacts from experimental environment. The value $f_{M A}$ was considered a constant among different experiments as long as the experiment was always taken place at the same experimental setup. This was because $f_{M A}$ represents the frequency of the resonance vibration and/or its harmonics, which was subject to the nature of the architectural structure and material of the building.

On the other hand, motion artifacts also can be generated by a patient and an operator of ultrasound system, such as the unavoidable body/organ movements of the patient and the probe movement if it was handheld by the operator. Although the occurrence and duration of those movements were unpredictable, the frequencies of common physiological activities, such as the muscle and organ motions, were relatively low and assumed to be measureable by our ultrasound system without aliasing.

As a result, the $f_{M A}$ could either be measured or be predicted, thus the SW displacement can be ideally isolated from those motion artifacts by using a temporal frequency filter on the measured tissue displacement, as long as the $f_{S}$ was separable from the $f_{M A}$ of those motions. However, the temporal frame rate $\left(f_{F R}\right)$ of the B-mode scanning was $30 \mathrm{~Hz}$ in our experiment, while the employed $f_{S}$ was typically above $30 \mathrm{~Hz}$. The temporal sampling of the SW displacement with such $f_{F R}$ belonged to an undersampling, which means, from the frequency spectrum analysis, the measured $f_{S}$ (termed $f_{\text {measured }}$ ) was aliased. Mathematically, the relationship between $f_{\text {measured }}$ and $f_{S}$ with respect to $f_{F R}$ 
was expressed by Eq. (5.2) [100]. More descriptions on undersampling were given in the Appendix C.

$$
f_{\text {measured }}=\left|n \cdot f_{F R}-f_{S}\right|
$$

where $n$ is the closest integer multiple to make $f_{\text {measured }}$ less than the Nyquist frequency $\left(1 / 2 f_{F R}\right)$

Therefore, the method to reduce the motion artifact was designed based on a temporal frequency filtering technique. The flowchart of the processing procedure for such task was depicted in Fig. 5.1.

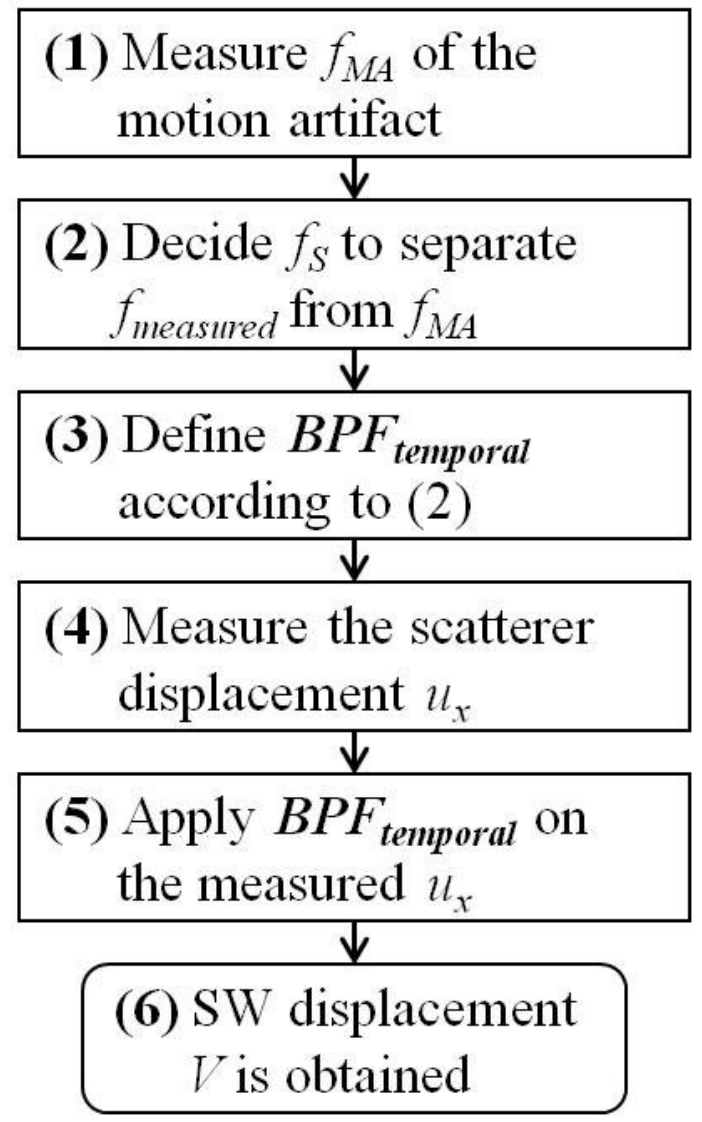

Figure 5.1. Flowchart of signal processing procedure to reduce the motion artifact. 
First, the $f_{M A}$ was estimated, and then $f_{S}$ was intentionally assigned to a specific value to avoid the $f_{\text {measured }}$ being overlapped with any of the applicable $f_{M A}$. Next, a temporal bandpass filter $\left(\boldsymbol{B P F}_{\text {temporal }}\right)$ was designed to retrieve the signals at $f_{\text {measured }}$. Finally, by applying such filter on the observed displacement $u_{x}$ with respect to the $t$ over all the locations in FOV, the displacement caused by the motion artifact would be reduced and the SW displacement $V$ will remain. Such process was expressed by Eq. (5.3), and after this process, $v_{S}$ would be calculated through Eqs. (4.4)-(4.7) as presented in Chapter 4.

$$
V(t)=\boldsymbol{B} \boldsymbol{P} \boldsymbol{F}_{\text {temporal }}\left\{u_{x}(t)\right\}
$$

It should be noted that the ultrasound probe only acquired signal along $x$-direction according to the experimental setup, thus theoretically the $\boldsymbol{B P} \boldsymbol{F}_{\text {temporal }}$ was effective to those displacements that occurred on $x$-direction.

\subsection{Experiments}

In order to verify the proposed method of motion artifact reduction, a phantom experiment was conducted. A soft tissue mimicking phantom was fabricated according to Section 3.3.2 by using $2-\mathrm{W} \%$ agar, $2-\mathrm{V} \%$ isopropanol (70\% purity), and $2-\mathrm{W} \%$ fine graphite particles. Ultrasound system and SW source used for the experiment were the same as those presented in Chapter 4.

Three measurement procedures were included in this experiment in order to conduct the motion artifact reduction. The first procedure was to detect the motion artifact caused by the measurement environment. The experimental configuration was 
seen in Fig. 5.2(a). The phantom was placed on a stable stage on an experimental table, while the ultrasound probe was held by a positioning device stably placed on the same table. No SW was introduced in this measurement, thus it was assumed that there was no relative movement between the probe and the phantom. Therefore, all the detected displacements, if applicable, would be considered as the motion artifact of the experimental environment, so the $f_{M A}$ from the environment could be found through this experiment.

The second procedure was to introduce the motion artifact intentionally caused by the human body movement. The experimental configuration was seen in Fig. 5.2(b). The ultrasound probe was held by a hand with no SW introduced. The handholding could cause unpredictable motions during the 6-second-long measurement, so such design was used in experiment to simulate an in-vivo condition where undesired patient/probe movement existed. Although the movement due to the hand-holding would not be identical among different measurements, it was assumed that the frequency range of the movement due to the hand-holding could be similar. Therefore, the $f_{M A}$ of the handholding could be roughly estimated through this measurement.

The third procedure was to measure the $v_{S}$. Ultrasound probe was held by hand in this measurement, and SW source was placed on the experimental table stably and the Plexiglas plate was contacted with the phantom and driven continuously by sinusoidal signal. The experimental configuration was seen in Fig. 5.2(c). Motion artifact was reduced according to the procedures presented in Fig. 5.1, and $v_{S}$ was estimated by using the modified $v_{S}$ measurement method presented in Chapter 4 . It should be noted that the experiment was conducted under the room temperature (around $20{ }^{\circ} \mathrm{C}$ ), and it was 
assumed that there were no $v_{S}$ changes due to temperature variation caused by the handholding.
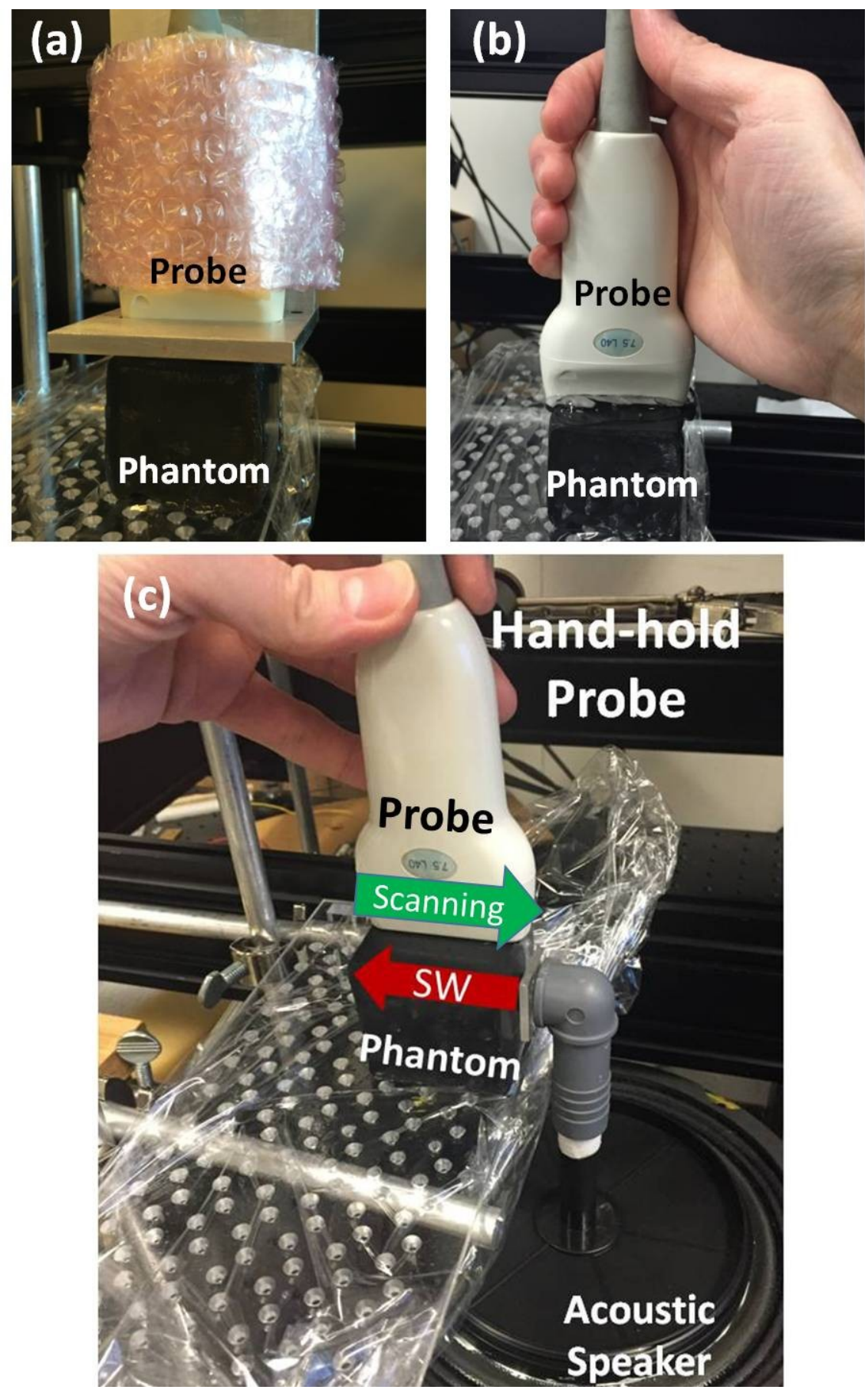

Figure 5.2. Experimental configuration for motion artifact reduction: (a) test without SW in a stage-holding probe design, (b) test without SW in a hand-holding probe design, and (c) SW velocity measurement in a hand-holding probe design. 


\subsection{Experimental Results}

\subsubsection{Motion Artifact Due to Experimental Environment}

Temporal frequency response of the tissue displacement in the first measurement procedure was obtained and averaged in the entire FOV. The result of the frequency spectrum was plotted in Fig. 5.3.

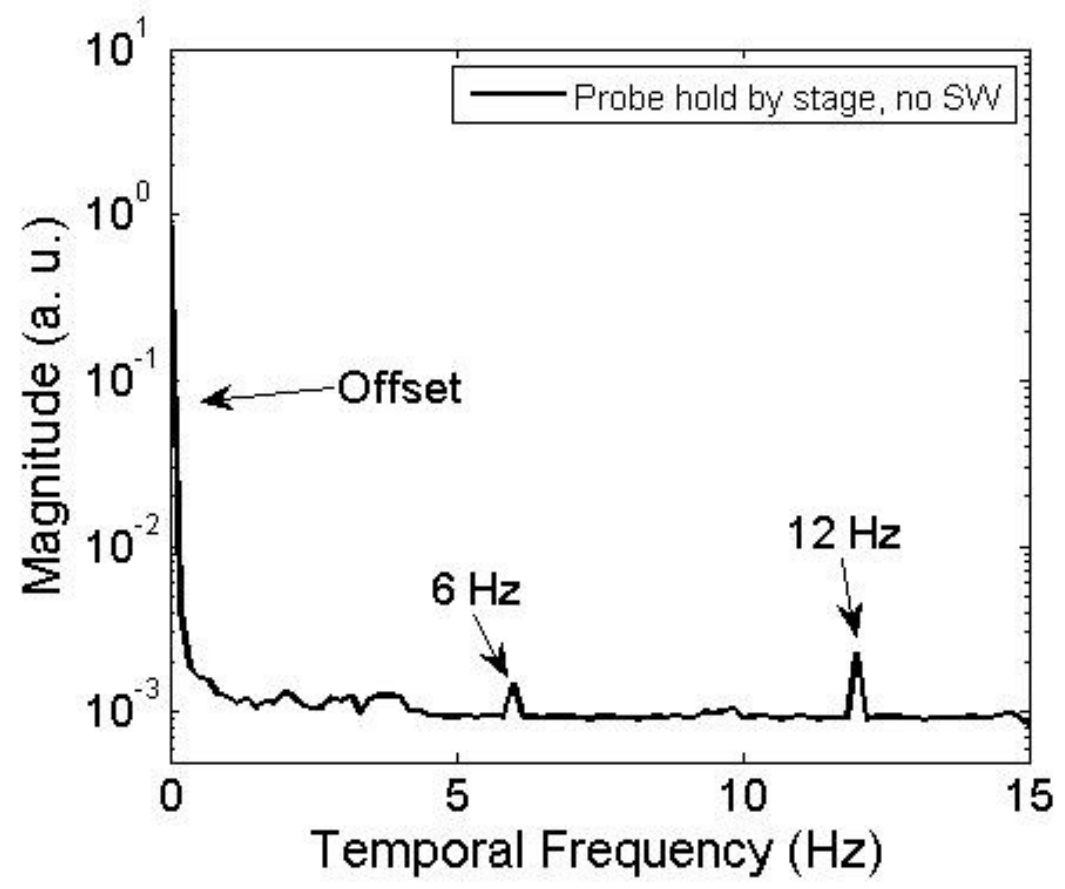

Figure 5.3. Temporal frequency spectrum of the first measurement procedure in which SW was not applied, and ultrasound probe was held by the positioning device.

Two noticeable frequencies of $6 \mathrm{~Hz}$ and $12 \mathrm{~Hz}$, and a zero frequency offset, were observed in Fig. 5.3. These frequencies were considered to be the $f_{M A}$ arising from the experimental environment of our laboratory. It should be noticed that these frequencies could be actual frequencies or aliased frequencies if the actual frequencies of the motions were greater than $15 \mathrm{~Hz}$ (as the Nyquist frequency of our system). 


\subsubsection{Motion Artifact Due to Handheld Probe}

Temporal frequency response of the tissue displacement in the second measurement procedure was obtained and averaged in the entire FOV. The result of the frequency spectrum was plotted in Fig. 5.4. The scale of the magnitude in this figure was the same as that of Fig. 5.3.

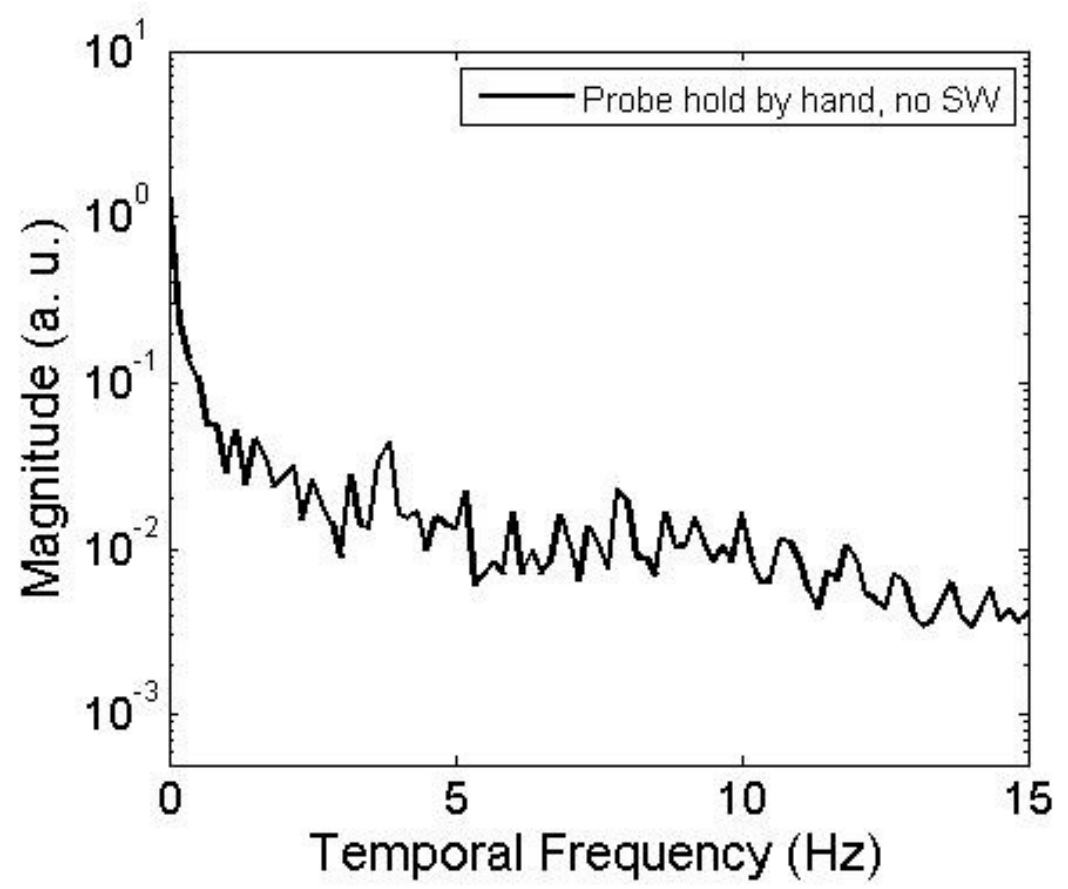

Figure 5.4. Temporal frequency spectrum of the second measurement ( $\mathrm{SW}$ was not applied, ultrasound probe was hand held).

The overall spectrum magnitude observed in Fig. 5.4 was stronger than that observed in Fig. 5.3, and the $f_{M A}$ in Fig. 5.4 covered the entire measurement range from 0 to $15 \mathrm{~Hz}$. Lower frequency components showed relatively stronger magnitudes than those in higher frequencies.

Based on the result in Fig. 5.3, $f_{S}$ and the passband of the $\boldsymbol{B P} \boldsymbol{F}_{\text {temporal }}$ can be decided. The $f_{S}$ was set to $227 \mathrm{~Hz}$, which would result in an $f_{\text {measured }}$ of $13 \mathrm{~Hz}$ with $n=8$. 
Then the $\boldsymbol{B P F}_{\text {temporal }}$ with a passband of $12.5 \mathrm{~Hz}$ to $14 \mathrm{~Hz}$ was designed, since no remarkable $f_{M A}$ in Fig. 5.3 was found and a relatively weaker magnitude in Fig. 5.4 was observed above $12 \mathrm{~Hz}$. It should be also noted that $227 \mathrm{~Hz}$ was selected because the amplitude of SW at such frequency was found stronger in our SW source, while the amplitudes of most other qualified frequencies may be too weak to be detected in practice.

\subsubsection{Shear Wave Velocity Measurement}

Temporal frequency response of the scatterer displacement in the third measurement procedure was obtained and averaged in the entire FOV. The result of the frequency spectrum was plotted in Fig. 5.5. The scale of the magnitude in this figure was the same as that of Fig. 5.3 and Fig. 5.4.

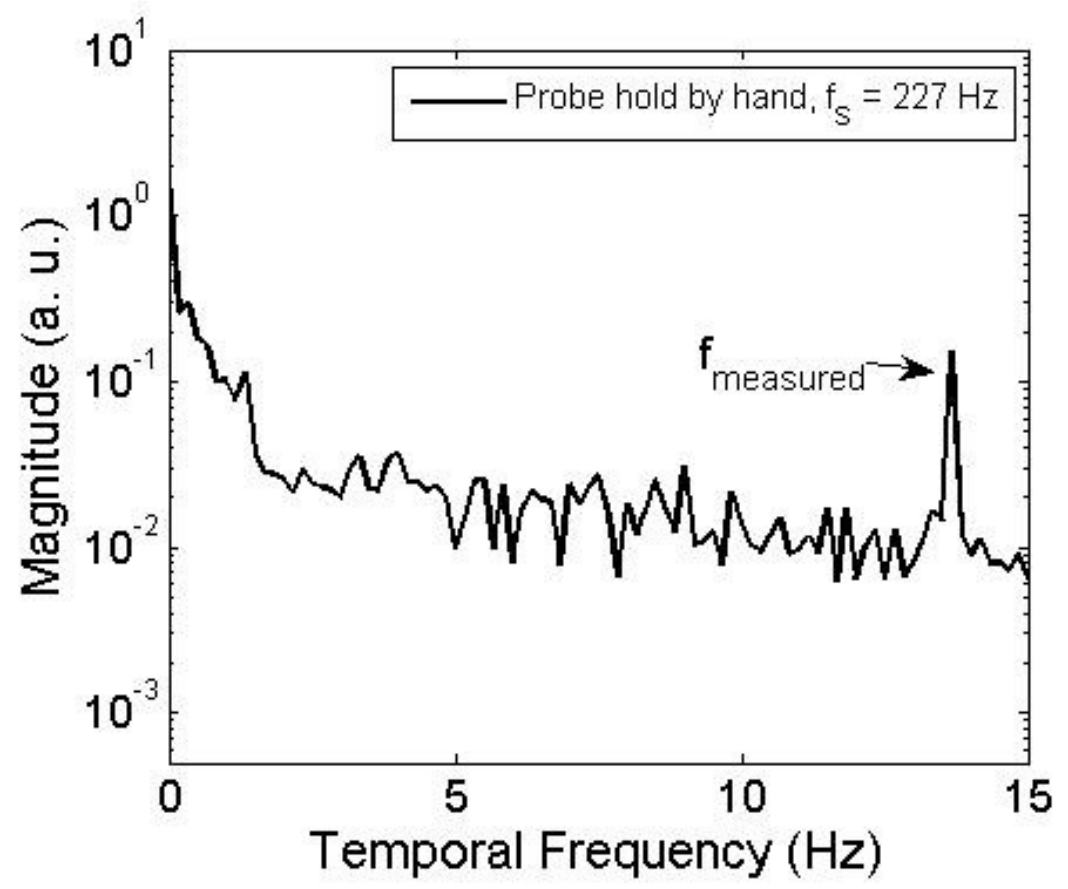

Figure 5.5. Temporal frequency spectrum of the second measurement (SW was applied, ultrasound probe was hand held). 
The $f_{\text {measured }}$ was observed at $13.7 \mathrm{~Hz}$, which was slightly different from the setting value of $13 \mathrm{~Hz}\left(f_{F R}=30 \mathrm{~Hz}\right.$ and $n=8$ in Eq. (5.2)). This was probably because small deviations existed between the set and actual value of $f_{S}$ in the practical experiment. Nevertheless, such value was still within the passband of $\boldsymbol{B P F}_{\text {temporal }}(12.5 \mathrm{~Hz}$ to $14 \mathrm{~Hz})$, so it would not affect the following calculations.

The motion artifact was reduced by using Eq. (5.3) with the $\boldsymbol{B P F}_{\text {temporal }}$ and the SW displacement $V$ would be obtained. An example of the observed SW displacement $V$ was shown in Fig. 5.6(a). The displacement along $z$-direction at the centerline $(x=0)$, indicated by the white dash-line in Fig. 5.6(a), was plotted in Fig. 5.6(b), and its spatial magnitude spectrum was shown in Fig. 5.6(c). In Fig. 5.6(c), two frequency components corresponding to $V_{F}$ and $V_{B}$ were noticed. The $\boldsymbol{B} \boldsymbol{P} \boldsymbol{F}_{\text {spatial }}$ was designed by following Section 4.2.2, and then the $V_{B}$ was retrieved from $V$ by the spatial frequency filtering. The filtered results were shown in Figs. 5.6(d)-(f).

If the process of motion artifact reduction was not applied, both $V$ and $V_{B}$ would contain more noise/error information, as presented in Figs. 5.7(a)-(f) with regard to Figs. 5.6(a)-(f), respectively.

The $v_{S}$ estimated with and without the reduction of motion artifact were presented in Figs. 5.8(a) and (b), respectively. Both results were averaged with 180 frames. The mean value of the $v_{S}$ in the ROI (as indicated by the dash-box) of Fig. 5.8(a) was calculated at $5.91 \mathrm{~m} / \mathrm{s}$ with a STD of $0.63 \mathrm{~m} / \mathrm{s}$, and those of Fig. 5.8(b) were $2.55 \mathrm{~m} / \mathrm{s}$ and $0.37 \mathrm{~m} / \mathrm{s}$, respectively. 

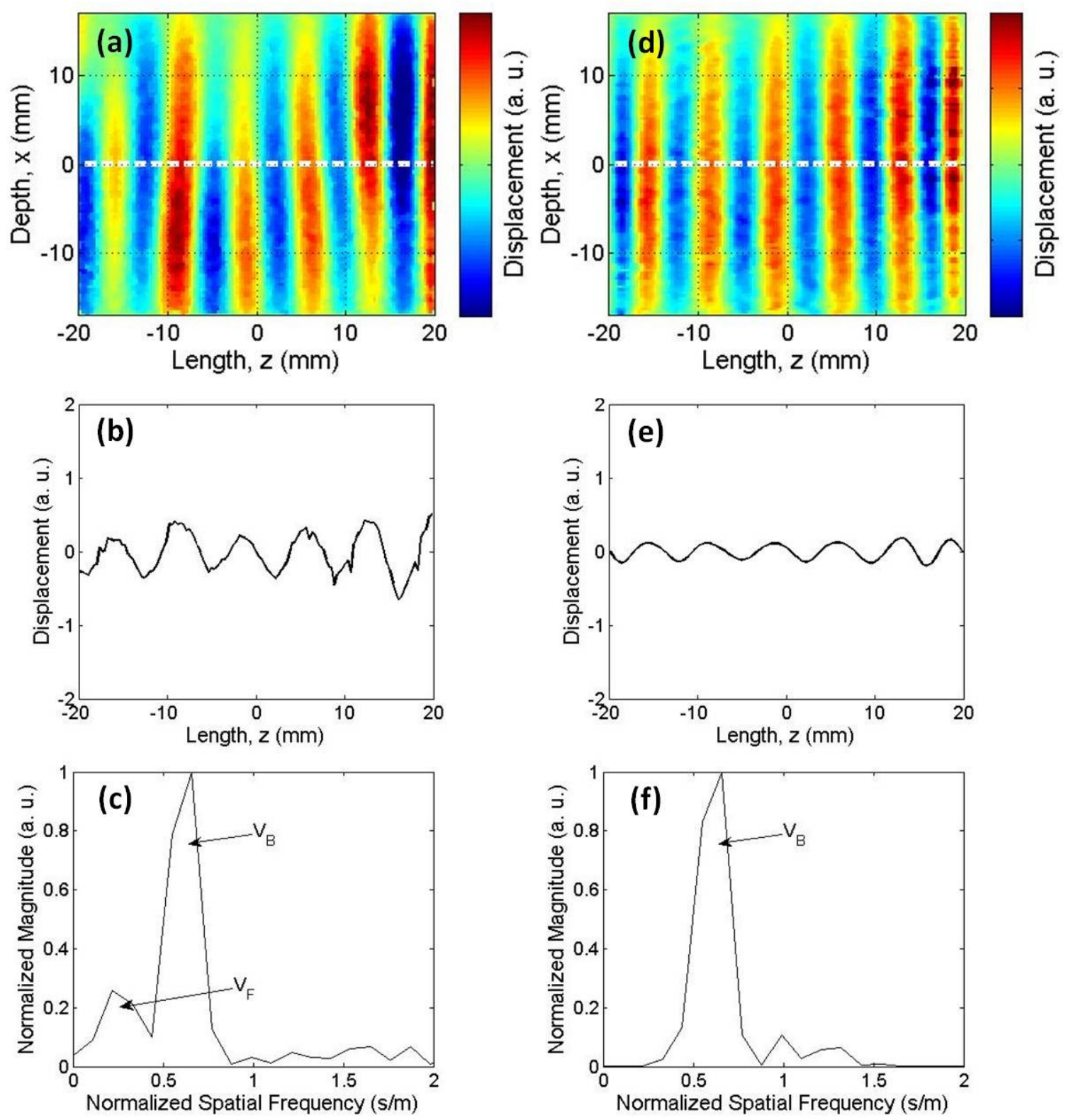

Figure 5.6. Observed SW displacements (a) in the entire FOV and (b) along $z$-direction at centerline $(x=0)$, and (c) magnitude spectrum of (b). And filtered SW displacements (d) in the entire FOV and (e) along z-direction at centerline, and (f) magnitude spectrum of (e). Results above were obtained with the reduction of motion artifact. 

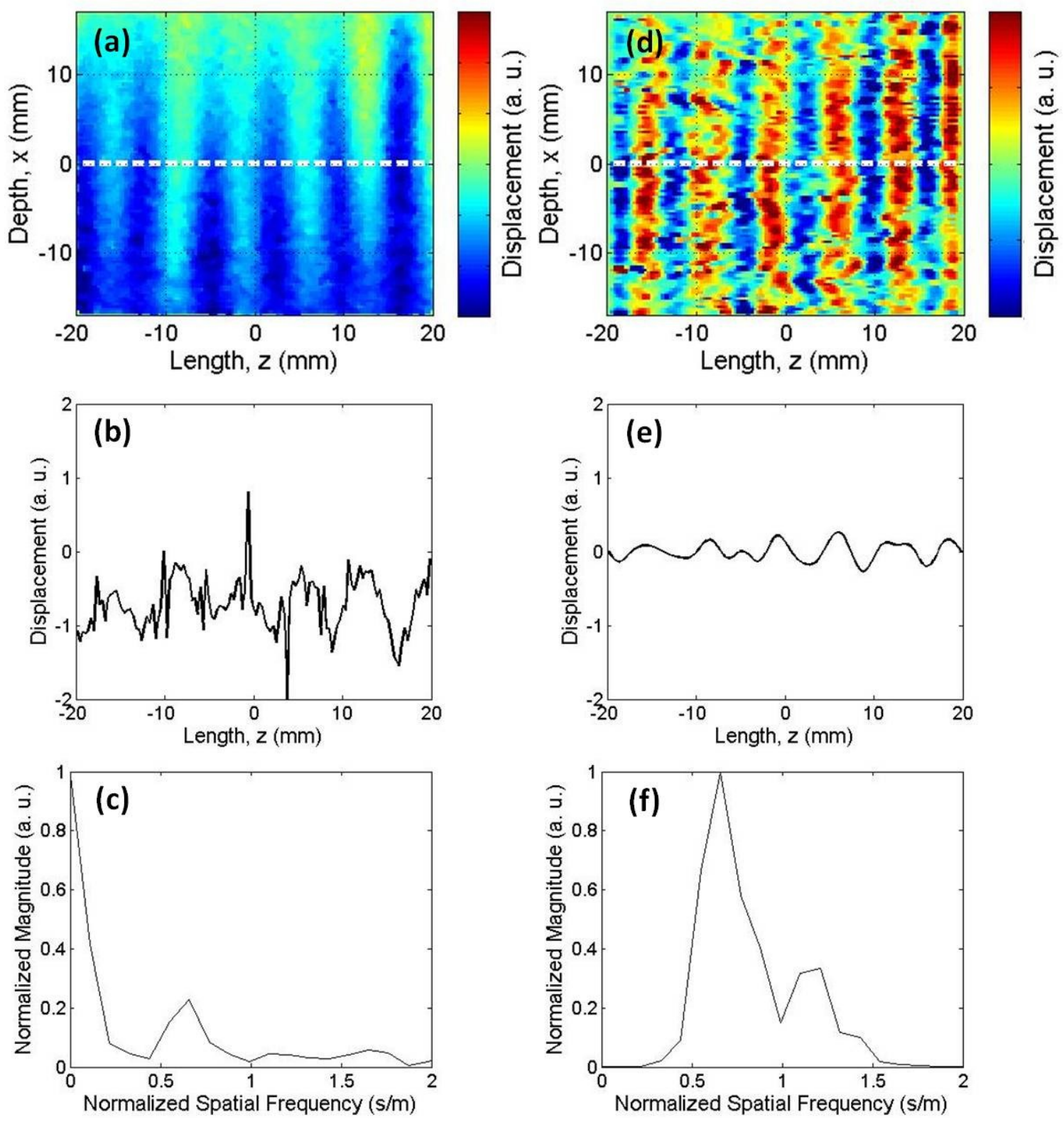

Figure 5.7. Observed SW displacements (a) in the entire FOV and (b) along $z$-direction at centerline, and (c) magnitude spectrum of (b). And filtered SW displacements (d) in the entire FOV and (e) along z-direction at centerline, and (f) magnitude spectrum of (e).

Results above were obtained without the reduction of motion artifact. 

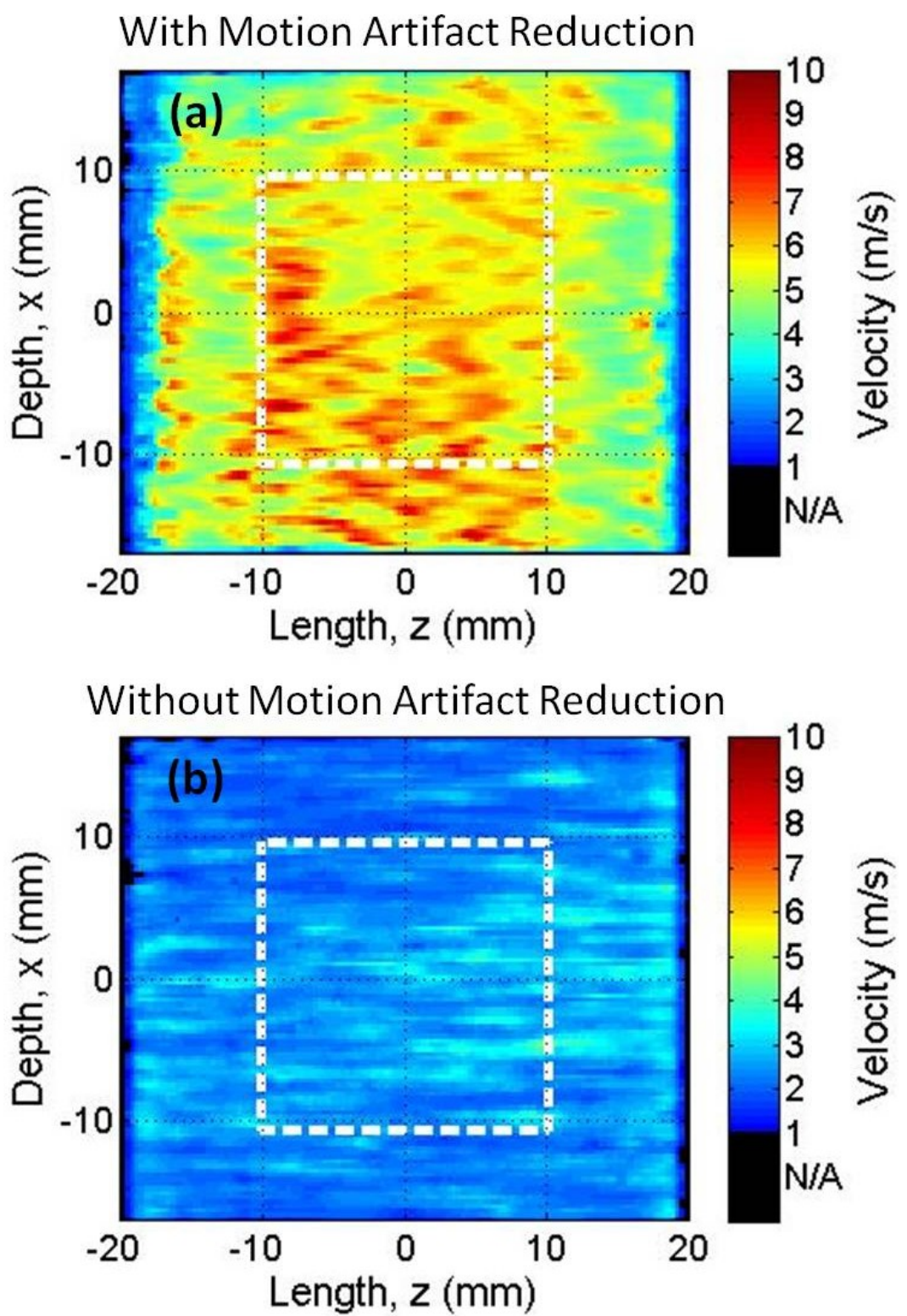

Figure 5.8. Results of SW velocity (a) with motion artifact reduction process, and (b) without motion artifact reduction process.

The $v_{S}$ estimated with the motion artifact reduction was consistent $(5.9 \mathrm{~m} / \mathrm{s})$ with that estimated from the phantom of the same composition and structure presented in Chapter 3, whereas the $v_{S}$ calculated without the reduction of motion artifact showed a great difference $(3.36 \mathrm{~m} / \mathrm{s}$ or $56.9 \%)$. In addition, the strongest $\mathrm{SW}$ amplitude measured in this experiment was less than $6 \mu \mathrm{m}$, while the amplitudes of the SW vibration in most 
of the published works which used external SW source were more than $100 \mu \mathrm{m}$ [7], [101], [102]. The smaller SW amplitude would be more desirable since it reduces the discomfort of the patient.

\subsection{Discussion}

The influence of the motion artifacts on the $v_{S}$ measurement was investigated by numerical simulations in this section. $V$ was simulated by using Eqs. (4.1), (4.2) and (4.3) with $v_{\text {scan }}=2.26 \mathrm{~m} / \mathrm{s}, v_{S}=1.02 \mathrm{~m} / \mathrm{s}$ to $10 \mathrm{~m} / \mathrm{s}, f_{S}=227 \mathrm{~Hz}$. The amplitude ratio $A_{F} / A_{B}=$ 1 was used to represent a total reflection and no SW attenuation was assumed. The FOV had the same size (40 $\mathrm{mm}$ in length and $34 \mathrm{~mm}$ in depth) as that in the phantom experiments. By considering that the $V_{M A}$ in Eq. (5.1) could be caused from multiple sources with different amplitudes and phases in practical experiments, it may be difficult to simulate $V_{M A}$ objectively. Therefore, a white Gaussian noise (WGN) was used to simulate $V_{M A}$ at any frequencies in the Eq. (5.1). WGN with a SNR at $10 \mathrm{~dB}, 20 \mathrm{~dB}$, and $30 \mathrm{~dB}$ were added into the simulations, respectively. It is noted that the values of the SNR chosen in this study may not be the actual ones in experiments. However, the purpose of the simulations was to investigate the effectiveness of the proposed motion artifact reduction method.

Two groups of simulations were conducted. In the first group, $\boldsymbol{B P} \boldsymbol{F}_{\text {temporal }}$ in Eq. (5.3) was defined to an all-pass filter, which means the process of motion artifact reduction was not conducted. Then in the second group, the process of motion artifact reduction was conducted. The same $\boldsymbol{B P F}_{\text {temporal }}$ as used earlier in Section $\mathbf{5 . 3}(12.5 \mathrm{~Hz}$ to 
$14 \mathrm{~Hz}$ ) was created and applied in Eq. (5.3). Then in each group, the $v_{S}$ over the FOV was calculated and smoothed by median filter. The mean values from the ROI (the same as those in Fig. 5.8) and STD of the $v_{S}$ calculated were shown in Fig. 5.9.
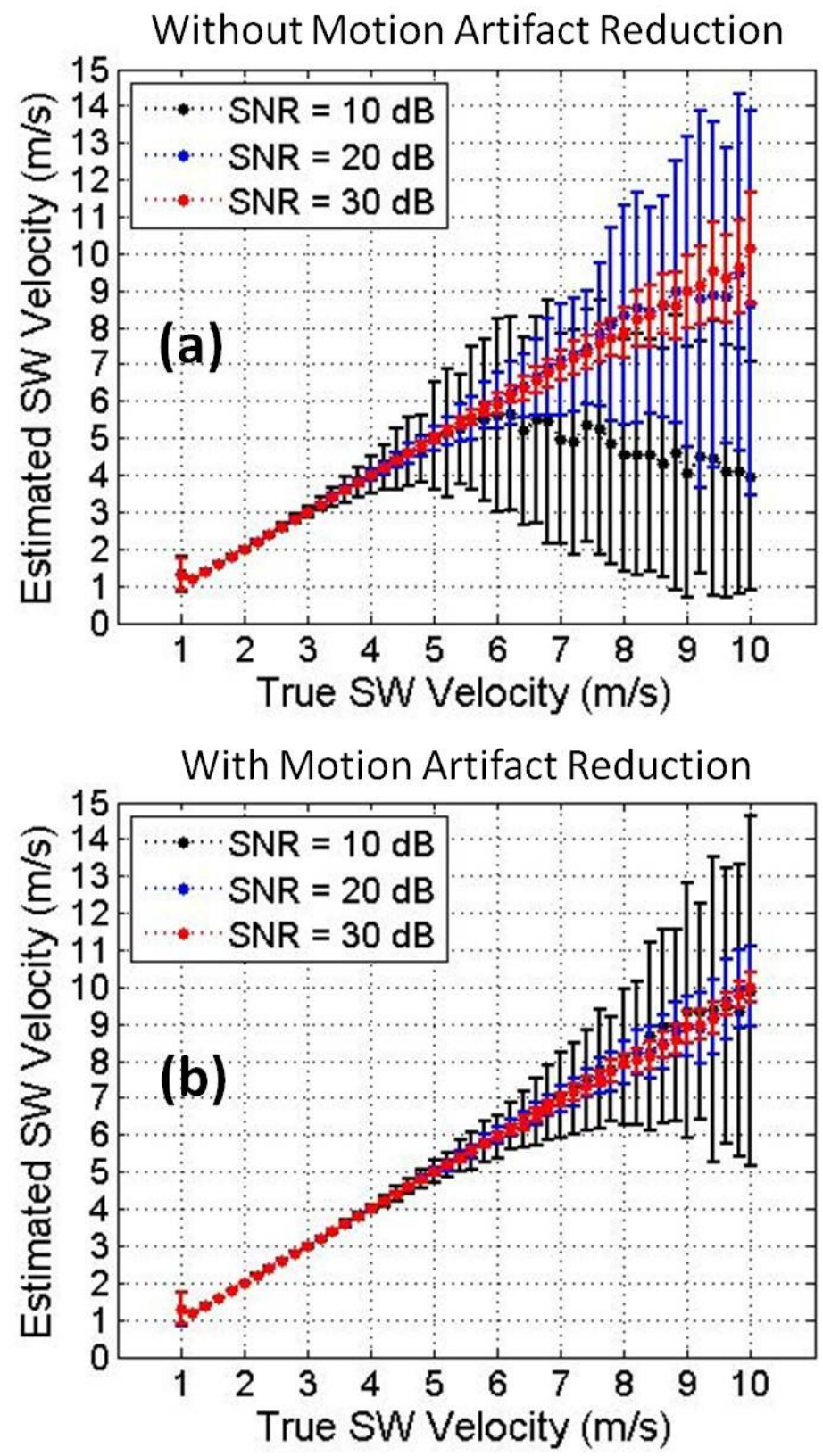

Figure 5.9. Calculated shear velocities versus to their actual values at different SNRs (a) without, and (b) with the process of motion artifact reduction, while black-dot-line represented the simulation with $10 \mathrm{~dB}$ WGN, Blue-dot-line represented $20 \mathrm{~dB}$, and reddot-line represented $30 \mathrm{~dB}$. 
Most of the mean values of estimated $v_{S}$ agreed with their set value (errors were less than $\pm 5 \%$ with the exception of $v_{S}=1.02 \mathrm{~m} / \mathrm{s}$ and $10 \mathrm{~m} / \mathrm{s}$, whose errors were less than $\pm 30 \%)$ except for the simulation that used $10 \mathrm{~dB}$ WGN without applying the motion artifact reduction. In that simulation result the estimated $v_{S}$ started to deviate from its set value when $v_{S}$ was approximately greater than $6 \mathrm{~m} / \mathrm{s}$. It can be seen that at a certain $v_{S}$ value and SNR, the estimated $v_{S}$ had less STD when the motion artifact reduction was applied, which means there were smaller errors in the estimation. For all the simulations, the STDs became smaller as SNR gets bigger. However, it was also found that the STDs would become greater with respect to the $v_{S}$. This was because the $\xi_{B}$ did not linearly change with $v_{S}$ according to Eq. (4.9). The change of $\xi_{B}$ with respect to the $v_{S}$ was calculated and shown in Fig. 5.10. It can be seen that the absolute gradient $\left(\left|\mathrm{d} \xi_{F} / \mathrm{d} v_{S}\right|\right.$, as indicated by green-solid-line) of the curve was greater when $v_{S}$ was lower, and vice versa. Therefore, with a same amount of spatial frequency change $\Delta \xi_{B}$, the velocity changes $\left(\Delta v_{S}\right)$ calculated at the lower $v_{S}$ value $\left(\Delta v_{S_{-} L o w}\right.$, as indicated by blue-dot) were different from that calculated at the higher $v_{S}$ value $\left(\Delta v_{S_{-} H i g h}\right.$, as indicated by red-dot). The $\Delta v_{S_{-} L o w}$ was less than $\Delta v_{S_{-} H i g h}$, which means the measurement resolution of $v_{S}$ on specimens with relatively lower $v_{S}$ values would be better than those of specimens with relatively higher $v_{S}$ values. 


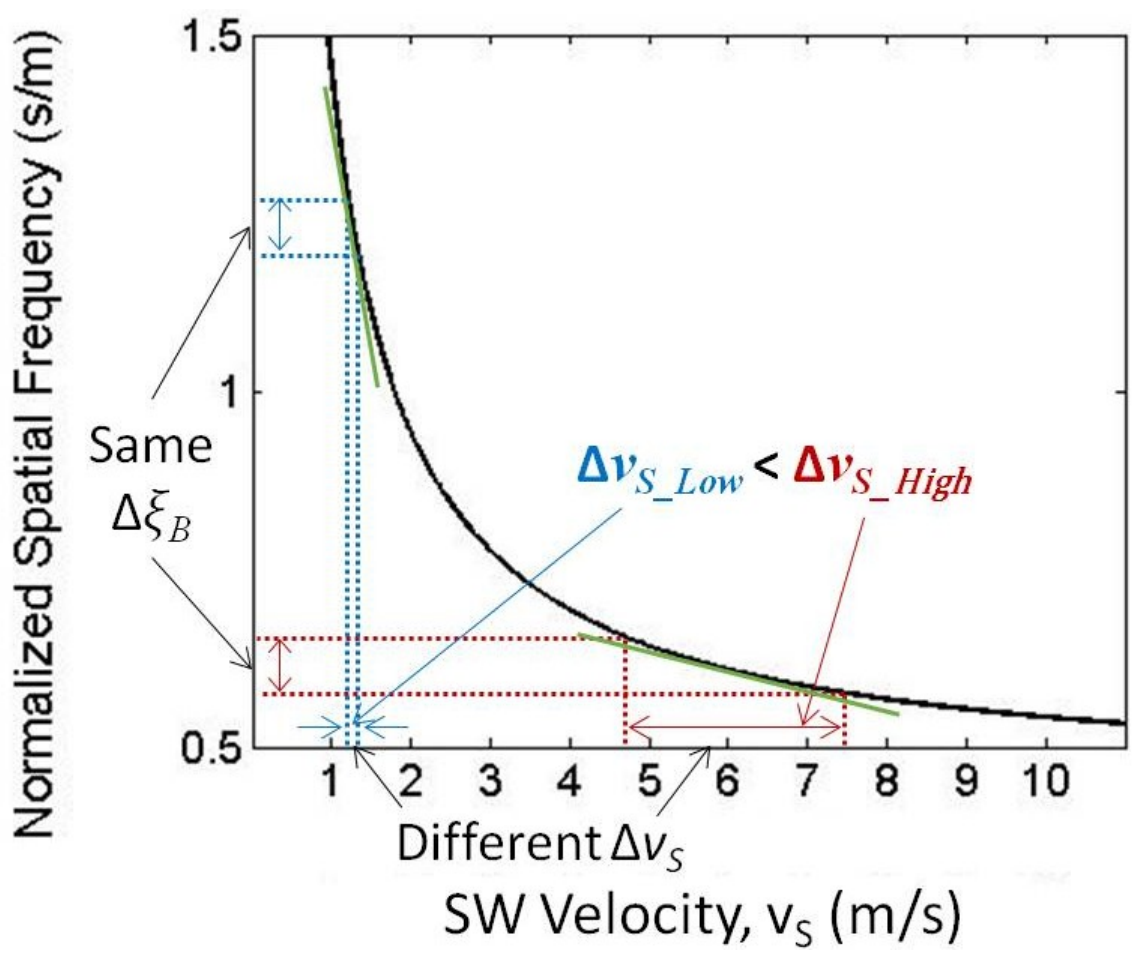

Figure 5.10. The change of the normalized spatial frequency of backward wave $\left(V_{B}\right)$ with respect to the SW velocity.

\subsection{Summary}

The research of this chapter addressed the motion artifact of the Problem 2 in Section 1.2. The method of the motion artifact reduction was proposed and applied based on the modified SW velocity measurement method presented in Chapter 4. The SW frequency was assigned to a specific value which was different from the frequencies of the predictable motion artifact so that the motion artifacts were reduced by applying a temporal bandpass filter on the measured tissue displacements. Phantom experiment was conducted to verify the proposed method and procedure. The performance of the method was verified by the numerical simulations. Therefore, the thesis Objectives 3 in Section 1.3 had been accomplished by the research of this chapter. 
The strongest SW amplitude measured in this experiment was less than $6 \mu \mathrm{m}$, while the amplitudes of the external SW vibration in most of the published works were more than $100 \mu \mathrm{m}$. The smaller SW amplitude would be desirable since it reduced the discomfort of the patient. The proposed method of motion artifact reduction in this chapter could be useful to lower the external SW vibration source power. 


\section{Chapter 6}

\section{In-vivo Measurement of Shear Wave Velocity}

\subsection{Introduction}

The measurement method of SW velocity was developed in the Chapters 3-4. A method of motion artifact reduction was proposed in Chapter 5, and the reduction of the motion artifacts would also enhance the accuracy of the estimated SW velocity. More importantly, such method was able to lower the external SW amplitude so that cause less discomfort to the patient. In this chapter, in-vivo experiments on human ankle and upper arm were conducted to demonstrate the proposed measurement methods for 2D SW velocity image.

\subsection{Experimental Setups}

Two in-vivo experiments were conducted. The first experiment was targeting at the $v_{S}$ measurement on the left ankle, and the second experiment was targeting at the upper limb of the author of this thesis, respectively. Experiments were conducted with a healthy male subject (age 32, weight $75 \mathrm{~kg}$ ).

The first experiment at ankle demonstrated the $v_{S}$ measurements of different types of in-vivo tissues. The reason to choose the ankle was because both heel tendon and 
skeleton muscle could be observed within the FOV (40 mm in length and $34 \mathrm{~mm}$ in depth) of the ultrasound system employed, and they would have noticeable difference in the $v_{S}$ values [33], [79]. In this experiment, the leg and foot were kept relaxed. A configuration of this experiment was shown in Fig. 6.1. The linear ultrasound probe was held by a stage and attached to the skin above the heel tendon. The head of the plastic rod of the external $\mathrm{SW}$ source was in contact with the leg and about $2 \mathrm{~cm}$ away from the near edge of the ultrasound probe. The SW source was held by hand during the experiment and driven continuously by sinusoidal signals at $227 \mathrm{~Hz}$ from a signal generator. The B-mode scanning direction was from the heel to the popliteal fossa, which allowed the ultrasound probe to detect the SW propagating along the tendon and the muscle. No other intentional movements were involved during the experiment to minimize the noise.

The second experiment at upper arm demonstrated the $v_{S}$ changes of in-vivo muscle tissues due to the muscle contraction. The muscle activity could result in stiffness change and lead to a $v_{S}$ change in the target muscle. Therefore, a dynamic $v_{S}$ change could be observed due to muscle contraction. To achieve this, muscles in the upper arm were contracted and relaxed during the SW measurement. In this experiment, the arm was kept flexed and placed on a flat panel of a portable stage. A configuration of this experiment can be seen in Fig. 6.2. The ultrasound probe was fixed on the portable stage and attached to the upper arm above the biceps. The head of the plastic rod of the external SW source was in contact with the upper arm, close to the elbow and about $5 \mathrm{~cm}$ away from the near edge of the ultrasound probe. The external SW source was held by a hand and driven continuously at $227 \mathrm{~Hz}$. During the experiment, the upper arm was fixed (moderately clamped) by the probe and panel to minimize involuntary motions from the 
body. Soft foam was placed between the panel and arm to make the arm comfortable. The B-mode scanning direction was from the shoulder to the elbow, which allowed the ultrasound probe to detect the SW propagating along the muscle fibers. At the beginning of the experiment, the bicep muscle was kept voluntarily contracted for a few seconds and then relaxed for the rest of the experiment.

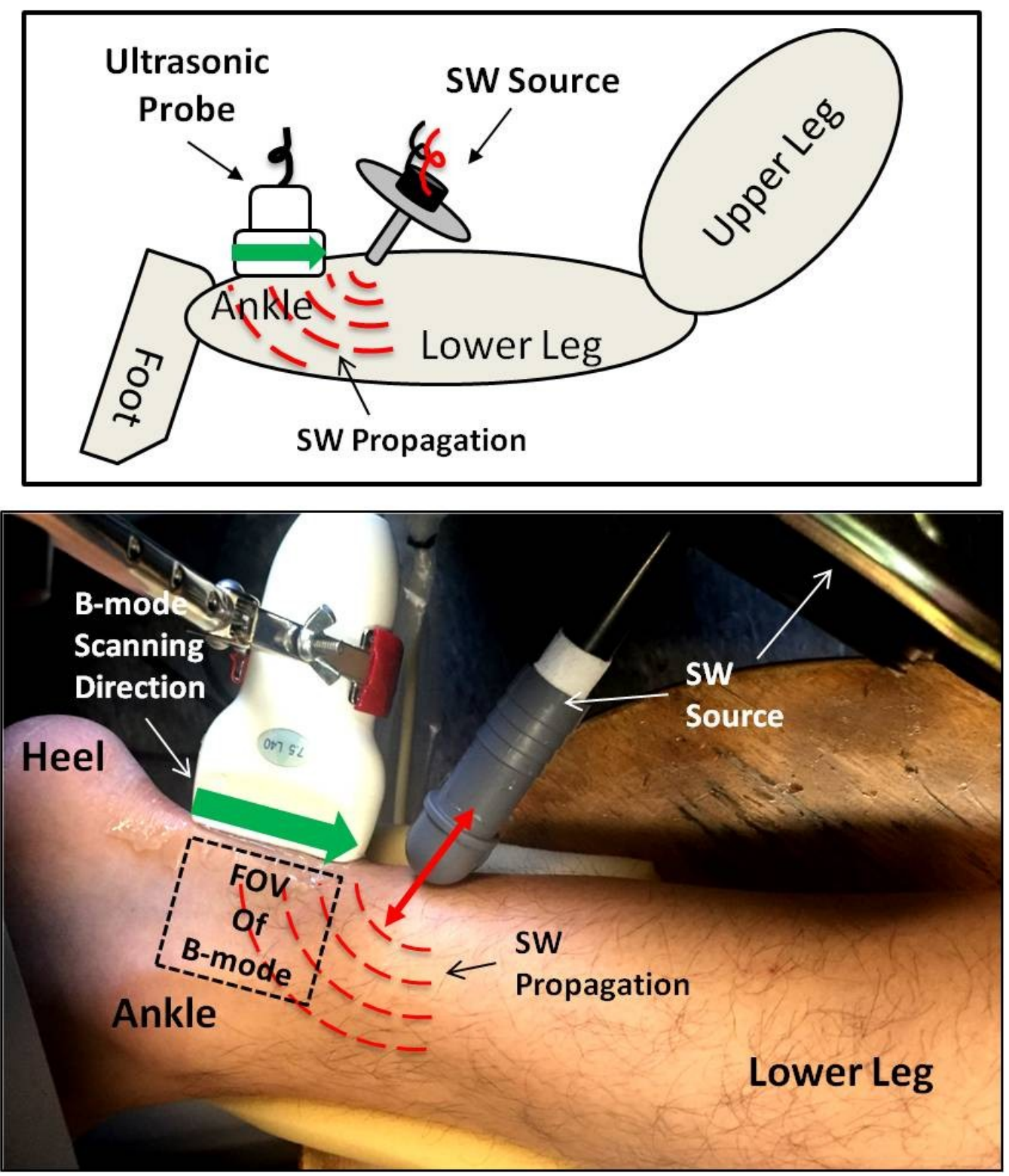

Figure 6.1. A schematic (top) and photograph (bottom) of the experimental configuration of shear wave velocity measurement at ankle. 


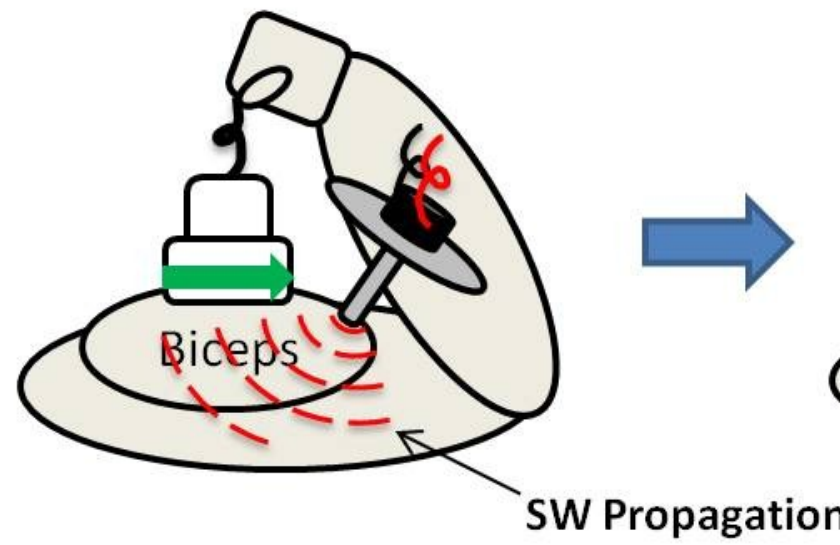

Contracted

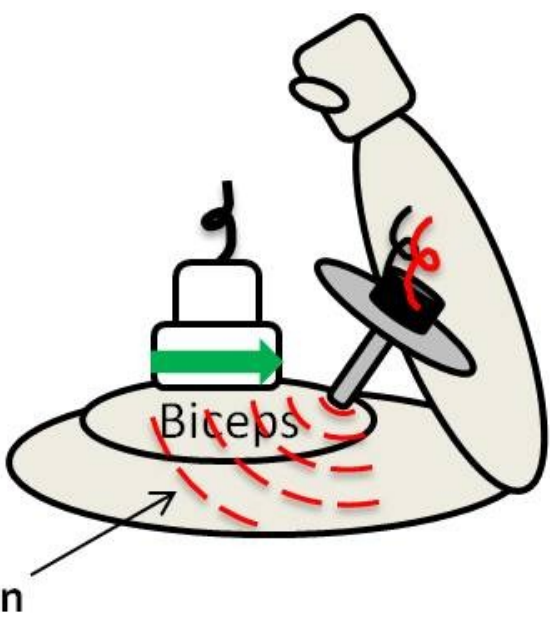

Relaxed

Figure 6.2. Experimental configuration of shear wave velocity measurements at upper arm.

It should be noted that the experimental configuration of both in-vivo experiments were not strictly the same as that of the phantom experiment presented in Fig. 4.1. SW source in the in-vivo experiments were located beside the ultrasound probe due to the limitation of the practical condition. Nevertheless, the round surface of the SW source generated SWs into a fan-shaped area (as indicated by the red ripples in Figs. 6.1 and 6.2), thus partial SWs would propagate through z-direction and they were observed by the ultrasound probe. The optimized location of probe and/or SW source for in-vivo experiment could be investigated in the future research. 


\subsection{Results and Discussions}

\subsubsection{Ankle Experiment}

Temporal tissue displacements due to SW propagation were analyzed. Their temporal frequency spectrum averaged in the entire FOV was plotted in Fig. 6.3. The $f_{\text {measured }}$ was found at $13.7 \mathrm{~Hz}$ and had a $0.7 \mathrm{~Hz}$ shift to its expected $13 \mathrm{~Hz}$ estimated from the under sampling $\left(f_{F R}=30 \mathrm{~Hz}\right.$ and $n=8$ in Eq. (5.2)) of the $\mathrm{SW}$ at $f_{S}$ of $227 \mathrm{~Hz}$. This frequency shift was consistent with the result in Fig. 5.5. In order to reduce the motion artifacts and noises within the passband of $\boldsymbol{B P} \boldsymbol{F}_{\text {temporal }}$, the passband of $\boldsymbol{B P} \boldsymbol{F}_{\text {temporal }}$ was set from 13.5 $\mathrm{Hz}$ to $14 \mathrm{~Hz}$ in this chapter.

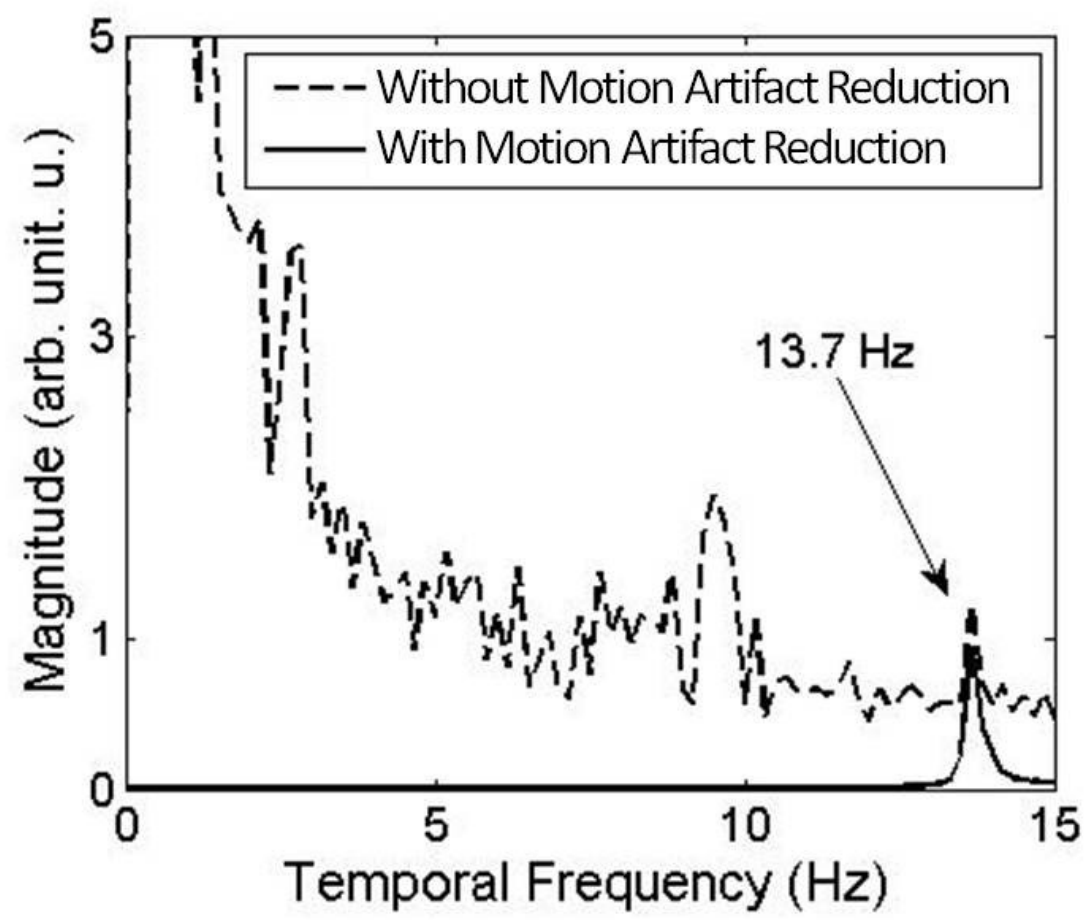

Figure 6.3. Temporal frequency spectrum averaged in the entire field-of-view. The dashline represented the spectrum before the motion artifact reduction and the solid-line represented the spectrum after the motion artifact reduction. 
The B-mode image, $v_{S}$ image with an average of 180 frames, and a superposition of B-mode image and $v_{S}$ image, obtained from the ankle experiment were presented in Figs. 6.4(a)-(c), respectively. The Achilles tendon was observed in the region indicated by the arrows in the B-mode image in Fig. 6.4(a). A clear $v_{S}$ image contrast was observed in Fig. 6.4(b) so that the $v_{S}$ were different between tendon tissue and muscle tissue. Two ROIs indicated by upper and lower white dash-boxes in Fig. 6.4(b) included the tendon tissue and muscle tissue, respectively. The estimated $v_{S}$ values below $1.02 \mathrm{~m} / \mathrm{s}$ or above $10 \mathrm{~m} / \mathrm{s}$ were considered as calculation error, and thus they were blackened and noted as N/A in Fig. 6.4(b). The averaged $v_{S}$ at the ROI of the tendon tissue was calculated 4.6 $\mathrm{m} / \mathrm{s}$ with a STD of $1.7 \mathrm{~m} / \mathrm{s}$, and those of muscle tissue were $2.5 \mathrm{~m} / \mathrm{s}$ and $0.75 \mathrm{~m} / \mathrm{s}$, respectively. The $v_{S}$ of tendon tissue was $2.1 \mathrm{~m} / \mathrm{s}(46 \%)$ greater than that of muscle tissue. From Fig. 6.4(c) it can be seen that the $v_{S}$ image contrast had a good correlation with the anatomical structure observed in the B-mode image. It is noted that the value of the $v_{S}$ dropped at the edges of the tendon area. This could be due to the calculation errors from the Hilbert transform. 


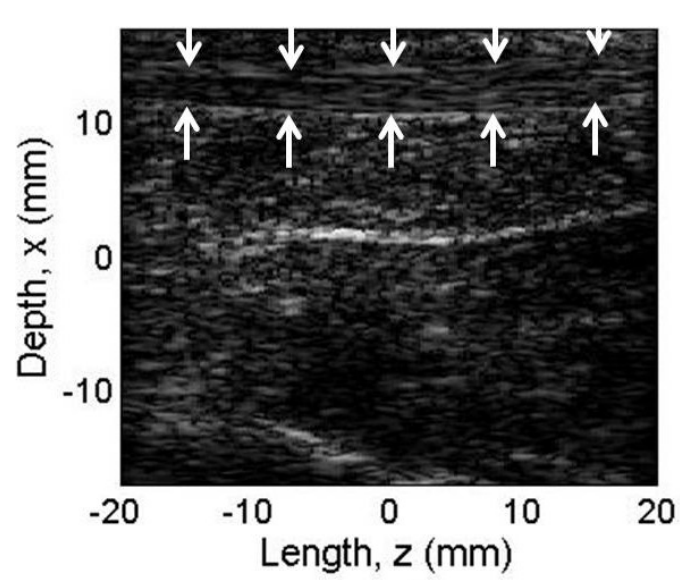

(a)

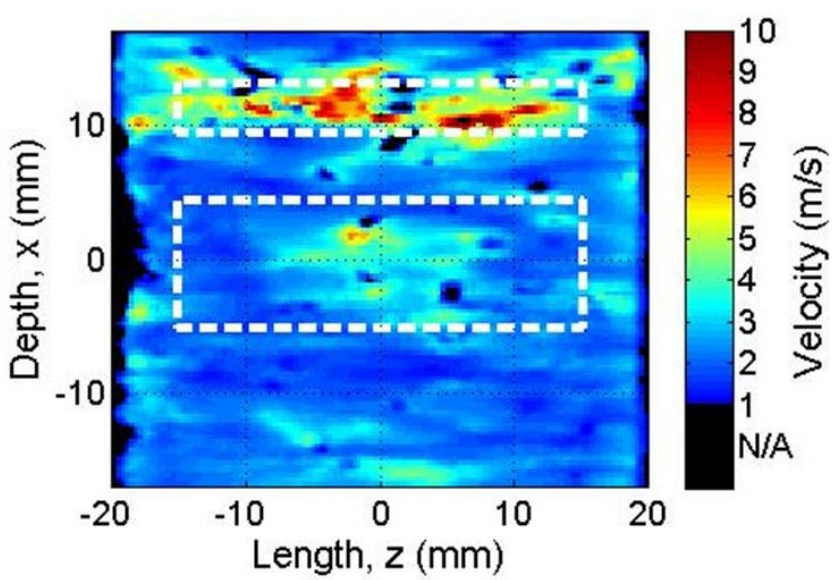

(b)

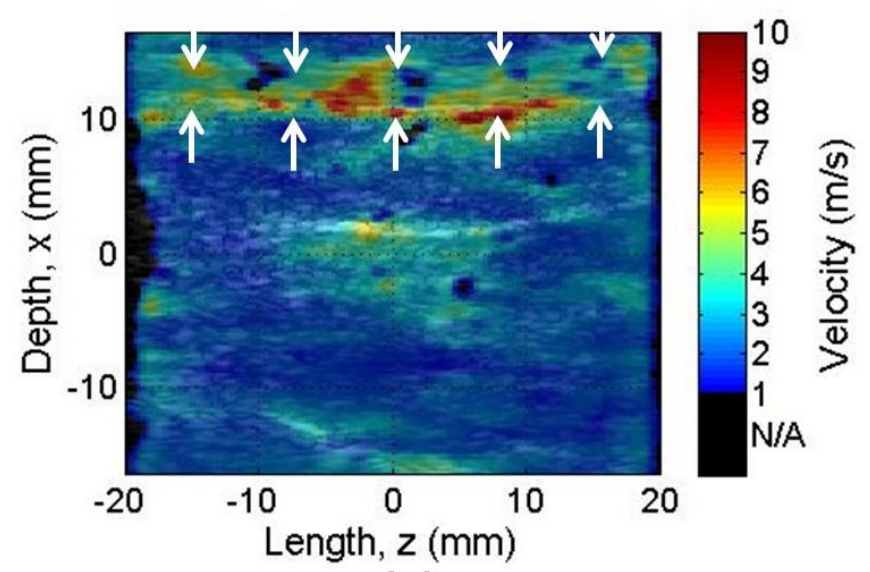

(c)

Figure 6.4. Results of ankle experiment: (a) B-mode image, (b) SW velocity image, and (c) a superposition of B-mode image and SW velocity image. Arrows indicated the Achilles tendon.

In order to investigate the stability of the $v_{S}$ measurements during the measurement time of 6 seconds, the mean values of the $v_{S}$ in the both tendon and muscle tissues, as indicated by the ROIs in Fig. 6.4(b), were calculated and averaged with every 10 consecutive frames. For instance, $v_{S}$ at frame $\# 90$ was an average result of frames from \#86 to \#95. The results were plotted in Fig. 6.5 with respect to the frames. The results in tendon area were plotted in Fig. 6.5(a) and those in muscle area were plotted in Fig. 6.5(b). 


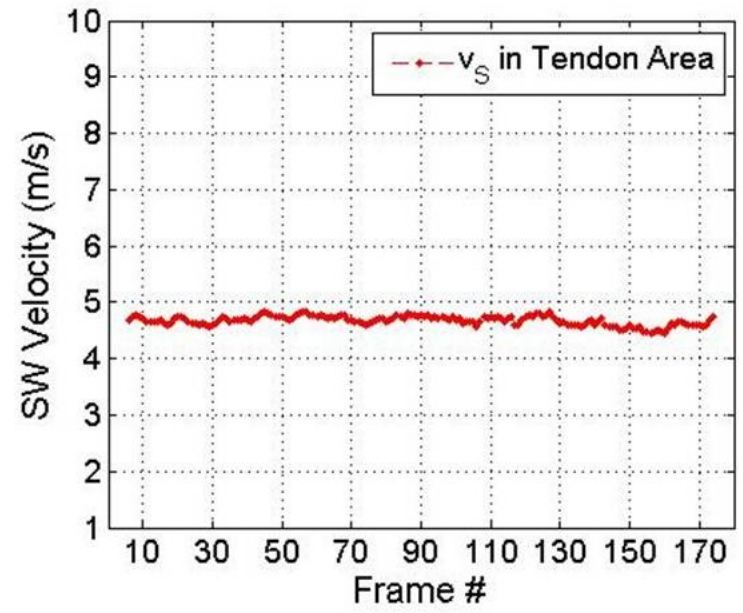

(a)

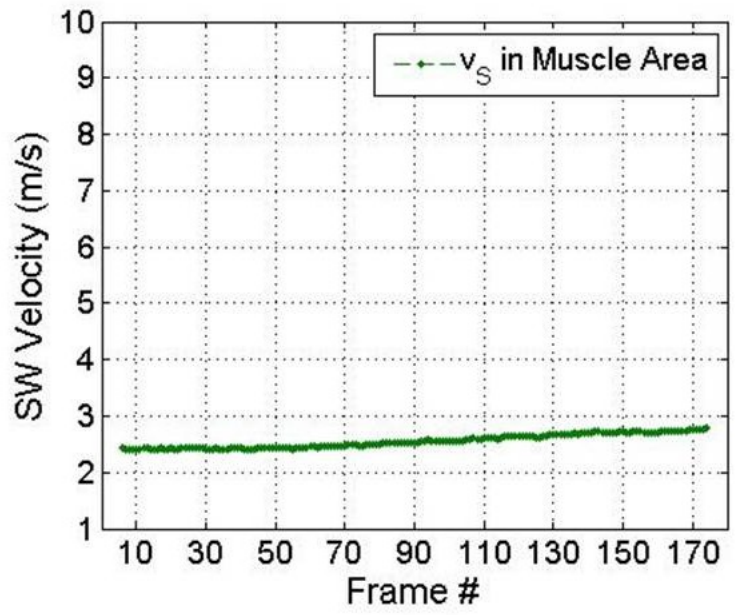

(b)

Figure 6.5. The change of the mean value of the SW velocity with respect to the temporal frames within the ROIs as indicated by the dash-box in Fig. 6.4(b). The result in the ROI of tendon was plotted in (a) and that of muscle was plotted in (b).

From Fig. 6.5 it can be seen that the $v_{S}$ variation (twice of the STD) in Fig. 6.5(a) was $0.17 \mathrm{~m} / \mathrm{s}$ and that in Fig. $\mathbf{6 . 5 ( b )}$ was $0.24 \mathrm{~m} / \mathrm{s}$ based on the 10 -frame averaging. Averaging the $v_{S}$ result with more temporal frames could be a selection to further improve the measurement accuracy. The $v_{S}$ difference between tendon and muscle tissues was consistent throughout the SW measurement.

Although the $v_{S}$ contrast between different tissues was observed in the $v_{S}$ results, it should be noticed that these estimated values of $v_{S}$ were relatively less than some published results [33][79]. This was possibly because a practical $\boldsymbol{B P F}_{\text {spatial }}$ applied on the spatial signal was not an ideal rectangular filter, so transition zones existed around the cut-off frequencies in its spatial frequency domain. If the spatial frequency corresponding to the $v_{S}$ was located in the transition zone of the filter, it may be removed or attenuated

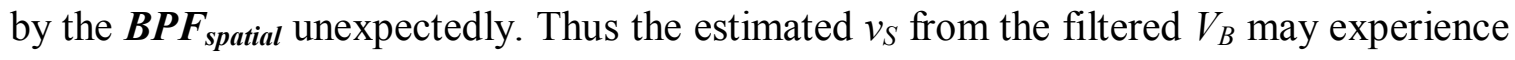


bias to its actual value. A frequency-domain filtering technique could potentially help to avoid such concern, and a brief analysis with such technique was discussed in Appendix D.

It was also noted that according to the discussion in Section 5.5, the absolute accuracy of the estimated $v_{S}$ could be varying among different tissues depending on the SNR within the $\boldsymbol{B P} \boldsymbol{F}_{\text {spatial }}$. Further study on the absolute accuracy of the estimated $v_{S}$ with the SNR could be a future research topic.

\subsubsection{Upper Arm Experiment}

In the upper arm experiment to monitor $v_{S}$ change of muscle due to muscle contraction, an alternative $\boldsymbol{B P} \boldsymbol{F}_{\text {spatial }}$ was assigned based on the frequency-domain filtering technique (see Appendix D). The passband of the alternative $\boldsymbol{B P F}_{\text {spatial }}$ was set from $0.54 \mathrm{~m}^{-1}$ to $0.94 \mathrm{~mm}^{-1}$ in order to measure $v_{S}$ between $2 \mathrm{~m} / \mathrm{s}$ and $10 \mathrm{~m} / \mathrm{s}$ since the $v_{S}$ values of muscles were typically in this range [21], [99], [101], [103]. Making the passband narrower helped to remove more noises, and thus improve the SNR of desired SW signals. B-mode image, observed SW displacement, filtered SW displacement, and $v_{S}$ image, respectively, at the selected frames \#10 (0.3 seconds), \#50 (1.7 seconds), \#90 (3.0 seconds), \#130 (4.3 seconds), and \#170 (5.7 seconds), were shown in Figs. 6.6(a)-(d), respectively. The $v_{S}$ image presented at each frame was averaged over every 10 consecutive frames.

The mean value of the $v_{S}$ in ROI, indicated by the dashed-rectangular in Fig. 6.6(d), were calculated at each frame, and plotted in Fig. 6.7. 


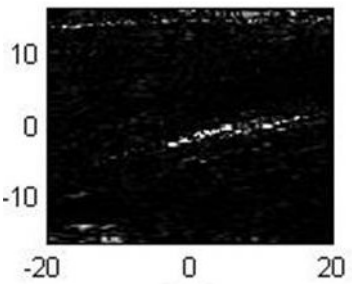

(a)

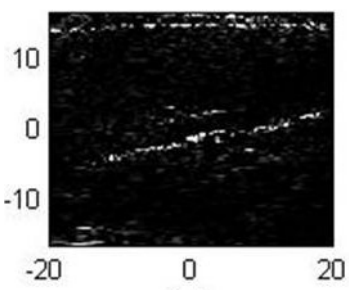

(a)

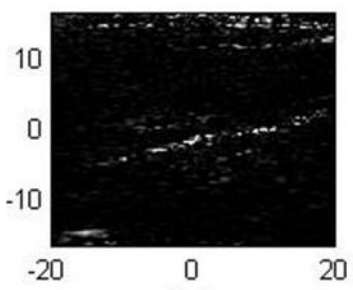

(a)

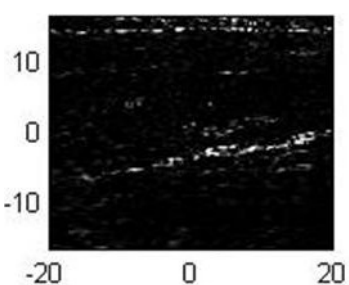

(a)

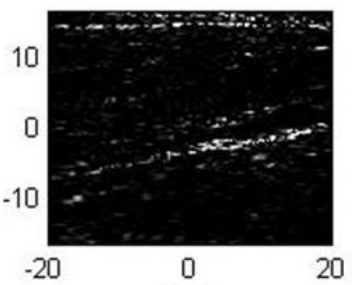

(a)

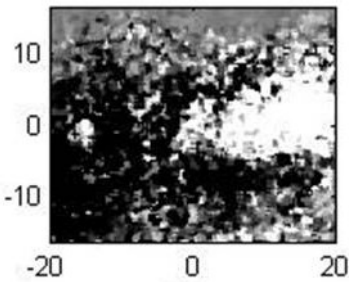

(b)

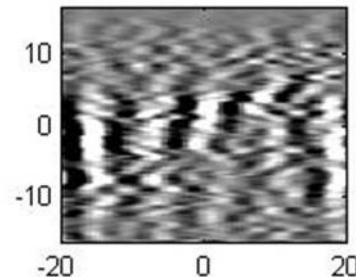

(c)

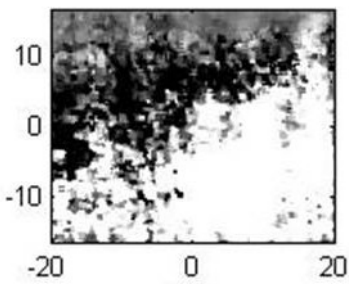

(b)

Frame \#50

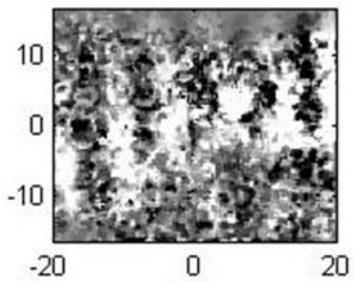

(b)

Frame \#90

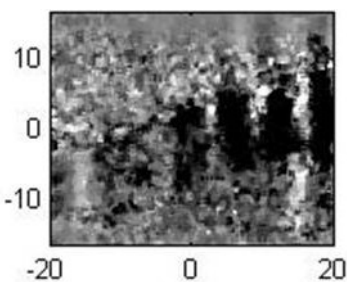

(b)

Frame \#130

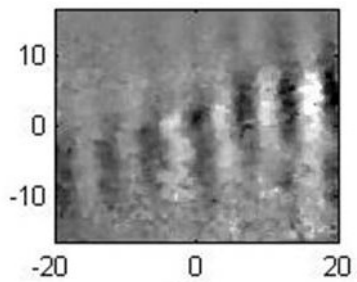

(b)

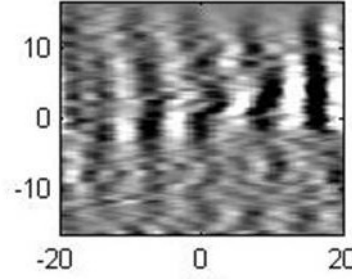

(c)

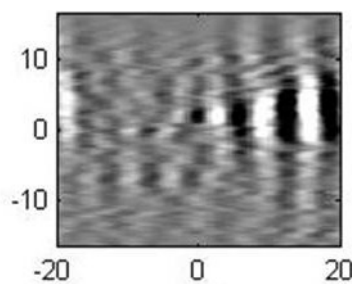

(c)

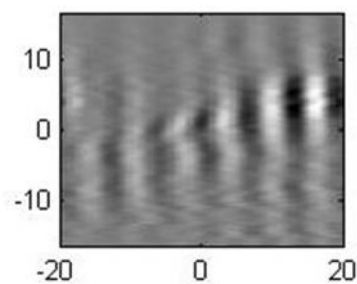

(c)

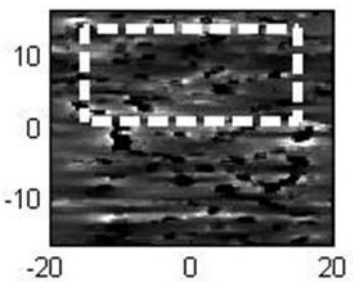

(d)

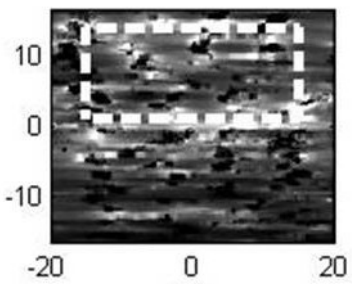

(d)

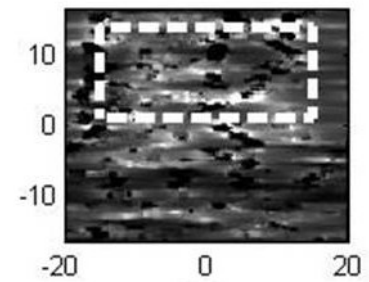

(d)

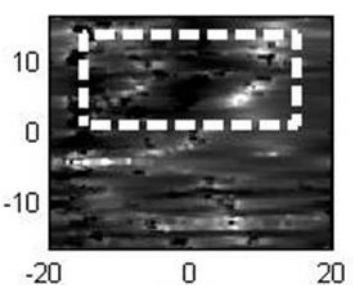

(d)

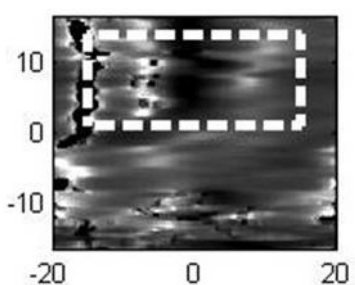

(d)

Figure 6.6. Results of arm measurement: (a) B-mode image, (b) observed SW displacement, (c) filtered SW displacement, and (d) SW velocity image. Dash-boxs in (d) were the ROIs to indicate the bicep muscle. Those results at frame \#10 (0.3 seconds after the start of the measurement), \#50 (1.7 seconds), \#90 (3.0 seconds), \#130 (4.3 seconds), and \#170 (5.7 seconds) were illustrated from top to bottom. 


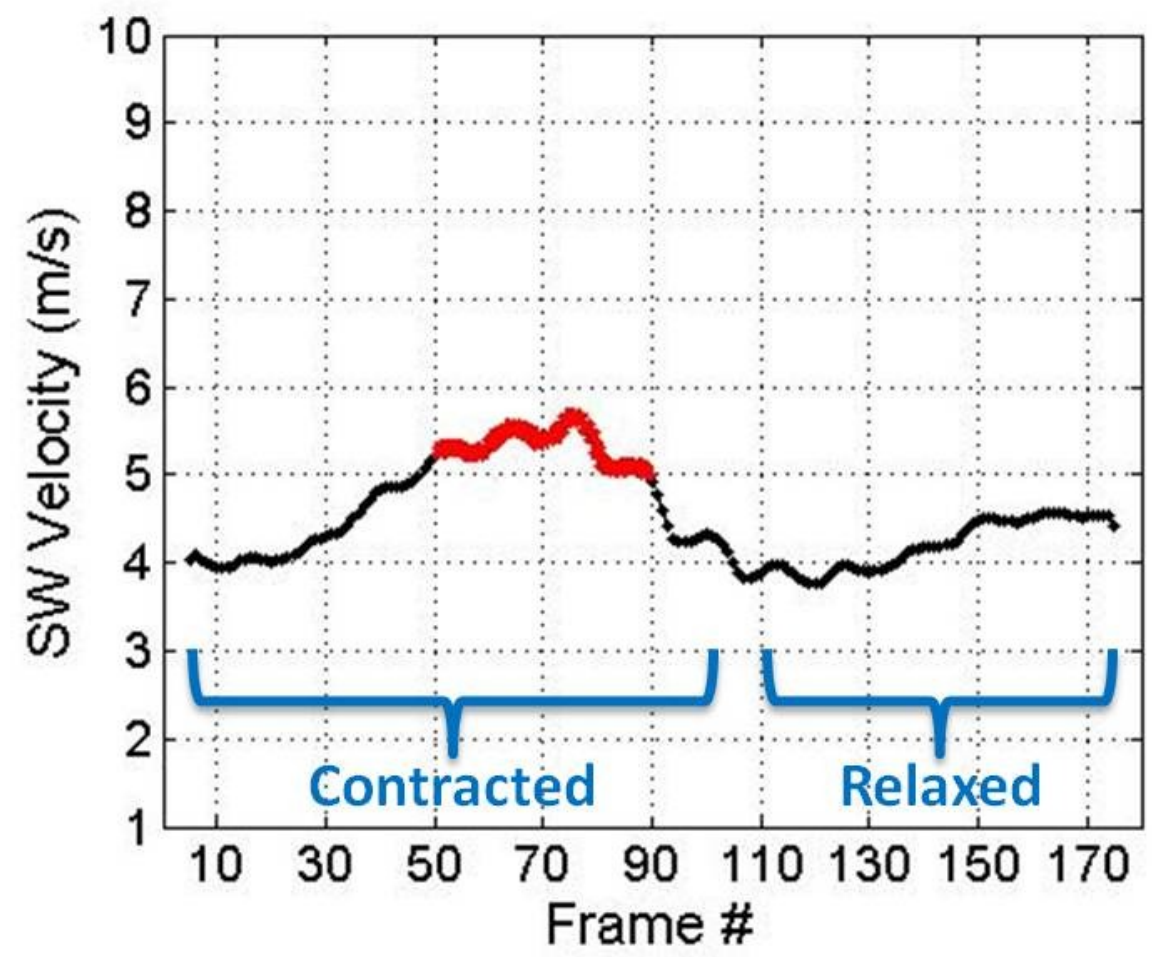

Figure 6.7. The change of the mean value of the SW velocity within the ROI in Fig. 6.6(d) with respect to the temporal frames.

It can be seen that the mean value of $v_{S}$ at bicep muscle was estimated above 5 $\mathrm{m} / \mathrm{s}$ between frame $\# 50$ and \#90 (as highlighted in Fig. 6.7), which was within the duration that the muscle was voluntarily contracted (approximately before frame \#100). This value became $4 \mathrm{~m} / \mathrm{s}$ to $4.5 \mathrm{~m} / \mathrm{s}$ when the muscle was totally relaxed (approximately after frame \#110). These results were close to the published results of contraction-relax measurement with bicep muscle [99], [101]. However, it was also noticed that the $v_{S}$ estimated before frame \#50 (when the muscle should be still contracted) had the similar value as those estimated when the muscle was relaxed. This may be because of the inconsistency of the muscle area voluntarily contracted during the experiment. Nevertheless, the $v_{S}$ change due to muscle contraction was dynamically observed with the 
proposed SW measurement method in Fig. 6.7, according to the results of the $v_{S}$ measurement stability at the relaxed muscle in Fig. 6.5.

\subsection{Summary}

In this chapter, the in-vivo experiments of SW velocity image were conducted using the $\mathrm{SW}$ velocity measurement method proposed in this thesis. The SW velocity difference between the muscle and tendon tissues at ankle was clearly observed. In addition, the change of the SW velocity of bicep muscle at upper arm due to muscle contraction was dynamically observed by using the proposed method. Since the SW velocity was related to the tissue stiffness which may reflect the tissue health, the proposed measurement method could be used to diagnose the muscle injury, and/or to evaluate the muscle strength/health level [12], [65], [67], [99], [104]. The measurement reproducibility could be improved with a stronger SW source which produces SWs with the better SNR. However, the patient discomfort may increase. 


\section{Chapter 7}

\section{Measurement of Shear Wave Absorption with}

\section{Correction of the Diffraction Effect}

\subsection{Introduction}

Shear elasticity and viscosity are two important parameters to characterize the mechanical properties of soft tissues. The methods to estimate the shear elasticity had been already implemented in some of commercial ultrasound imaging devices, while the viscosity measurements were still in research stage.

As described in Chapter 2, viscosity can be estimated either by an analysis on the dispersion of SW velocity with multiple SW frequencies, or through measuring both the SW velocity and absorption at a chosen frequency. There were various demonstrations to estimate the soft tissue viscosity by SW velocity dispersion analysis [17], [20], [76], [99], [105]. The advantage of such method was that it avoided the direct measurement of SW absorption, which was rather challenging to measure precisely. However, the dispersion method requires multiple measurements at different frequencies to determine the SW velocity dispersion unless using an impulse SW having a broad frequency bandwidth with a high frame-rate system [99]. The proposed SW measurement method in this thesis used a continuous SW at a chosen frequency. Thus the dispersion method required multiple measurements with our system used in this thesis. However, the prolonged 
measurement time may not be desired since it could increase the patient discomfort and the difficulty of the measurement reproducibility. Therefore, it was preferable to measure SW velocity and absorption simultaneously with a single same experiment.

In this chapter, a measurement method of SW absorption was proposed based on the SW attenuation measurement with a compensation of diffraction effect using the same measurement configuration of the SW velocity presented in Chapters 3, 4, 5 and 6 . Since precise measurement of SW absorption for a $2 \mathrm{D}$ absorption image was rather challenging, the objective of this chapter was to develop a measurement method to estimate an averaged SW absorption of the entire specimen. Therefore, it was assumed that a specimen was homogenous and isotropic. Although the proposed method in this chapter remained in a preliminary stage, it could provide a reference value of SW absorption of soft tissue. Such information could be useful for some clinical applications, such as a biopsies.

\subsection{Methodology}

\subsubsection{Measurement Model of Shear Wave Absorption}

SW attenuation was represented by the amplitude loss of a SW propagating a certain distance. When a SW propagation medium was homogenous and had an infinite size, the absorption, scattering and diffraction effect were the main factors of the SW attenuation. If the propagation medium had a finite size or had boundaries, additional factors such as reflection and mode conversion also changed the SW amplitude. In order to 
experimentally estimate the absorption from the measured $\mathrm{SW}$ attenuation, these factors needed to be taken into account.

In this chapter, it was assumed that a tissue specimen was homogenous and isotropic, and had a finite size. With these assumptions, scattering effects and conversion were negligible in our measurement configuration (to be discussed later). In addition, the SW reflected at the edge of the specimen can be removed by the method using a B-mode scanning time delay proposed in Chapters 3 and 4. Therefore, the absorption and diffraction were the main factors of the SW attenuation. The absorption converted the SW mechanical energy to heat [106]. The diffraction effect made an induced SW a divergent wave rather than a plane wave. It was also known that the diffraction nonlinearly affected the amplitude change in the spatial domain.

Figure 7.1 showed the SW absorption measurement configuration which was the same as the one used in SW velocity measurement in Fig. 4.1, except two modifications made in the measurement coordinate system. The origin of the coordinate system was moved to the center of the interface between the SW source and the specimen, and the direction of $z$-axis was the same as that of the backward SW. As discussed in Chapter 4, the induced SW displacement $V_{l}(z)$ can be retrieved from the observed SW displacement $V(z)$ using the B-mode scanning time delay. 


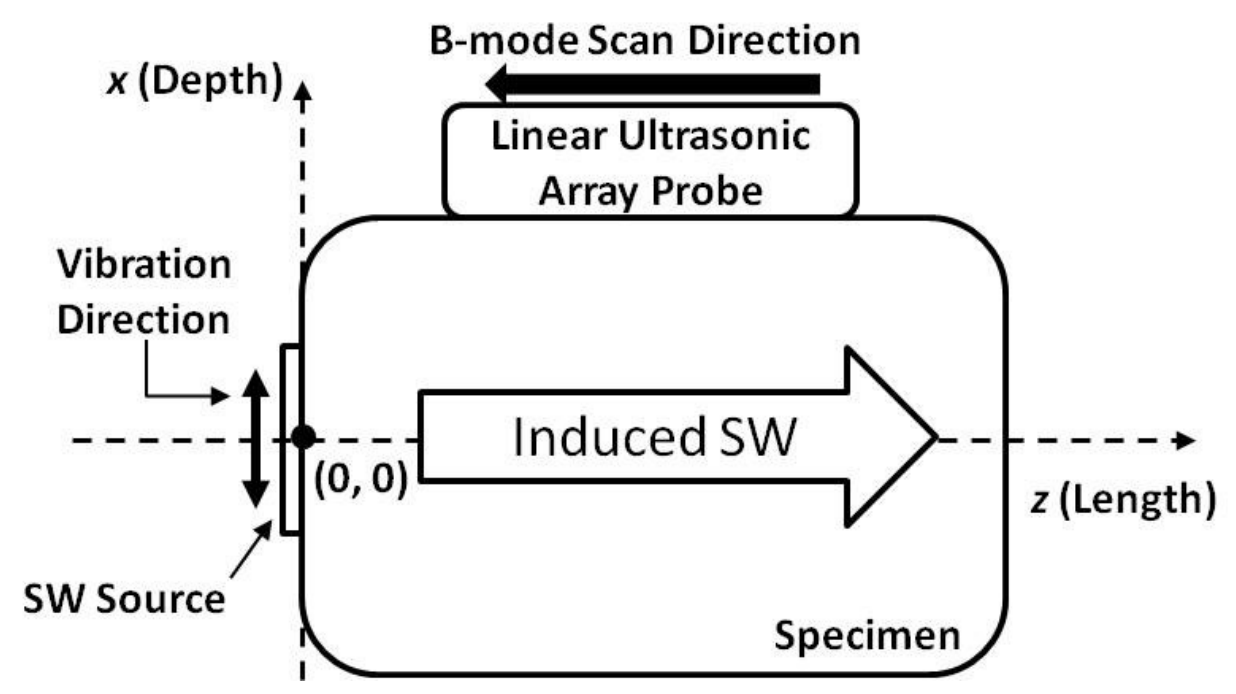

Figure 7.1. Measurement model of SW absorption.

According to the theory of Rayleigh scattering [107], [108], the amplitude of the scattered wave was proportional to the inverse fourth power of the wavelength and the sixth power of the scatterer's radius (assuming that the scatterer can be considered as spherule). Under a practical condition, the SW wavelength $\lambda_{S}\left(=v_{S} / f_{S}\right)$ was usually in a few centimeters, while the size of the scatterer in soft materials was usually as small as a few to a few tens of micrometers (e.g. the nominal diameter of graphite particle used in the phantom was $50 \mu \mathrm{m})$. So the size of scatterer was much less than the wavelength. Therefore, the scattering effect was considered to be negligible.

The energy of a propagated SW would be reduced due to a mode conversion when such wave experienced a reflection. However, even though a reflection may happened at the top and/or bottom edge of the specimen if the induced SW was diverged due to diffraction, it would only lead to a negligible energy loss to the $V_{I}$ from mode conversion (see Appendix A). Therefore, such factor was also neglected. 
According to the measurement configuration in Fig. 7.1, the induced SW $V_{I}(z)$ was expressed by Eq. (7.1). Noted that the Eq. (7.1) was equivalent to the backward SW $V_{B}(z)$ as presented by Eq. (4.3) in Chapter 4. The variation of amplitude $A_{l}(z)$ with respect to the $z$-direction was used to determine the SW absorption $\alpha_{S}$ of a viscoelastic tissue at a chosen frequency. $A_{I}(z)$ was expressed by Eq. (7.2).

$$
\begin{aligned}
V_{I}(z) & =A_{I}(z) \cos \left[-\omega_{S}\left(\frac{1}{v_{S}}+\frac{1}{v_{\text {scan }}}\right) z+\varphi_{I}\right] . \\
A_{I}(z) & =\left|V_{I}^{*}(z)\right| \\
& =A_{S} \cdot \operatorname{ATT}(z) \cdot e^{-\alpha_{S} z},
\end{aligned}
$$

where $\varphi_{I}$ is the phase of the $V_{I}(z) . V_{I}^{*}(z)$ is the complex induced SW signal calculated using Hilbert transform as presented by Eq. (4.5). $\operatorname{ATT}(z)$ is the attenuation due to the diffraction effect and $\alpha_{S}$ is the $\mathrm{SW}$ absorption coefficient. $A_{S}$ is an initial SW amplitude at the SW source $(z=0)$.

Figure 7.2 presented an example of the SW amplitude variations measured with a hydrogel phantom [109]. SW amplitude over the entire FOV, amplitude profile of the centerline (along $x$-direction at $\mathrm{x}=0$ ), and along $x$-direction at $z=12 \mathrm{~mm}$, were shown in Figs. 7.2(a), (b), and (c), respectively. A rectangular Plexiglas plate was used as the SW source which had dimensions of $30 \mathrm{~mm}$ in height, $15 \mathrm{~mm}$ in width, and $1 \mathrm{~mm}$ in thickness. The $f_{S}$ of the experiment was $322 \mathrm{~Hz}$. The $v_{S}$ was measured at $4 \mathrm{~m} / \mathrm{s}$. In this experiment, M-mode measurements were conducted repeatedly at different $z$ locations to map the SW displacement, instead of the B-mode measurement.

From Fig 7.2 it can be seen that the SW amplitude was the greatest at the area close to the SW source with the presented experimental configuration employed. The SW 
amplitude and beam width then gradually decreased along the $z$-direction due to the diffraction and absorption of the SW. The slight asymmetric amplitude with respect to the centerline was probably due to the inhomogeneity of the phantom material fabricated and/or alignment error of the SW source employed in the experiment. In addition, the observed amplitude along $x$-direction at $z=12 \mathrm{~mm}$ had an "M" shape profile but not the plane wave, due to the nonlinear diffraction effect.

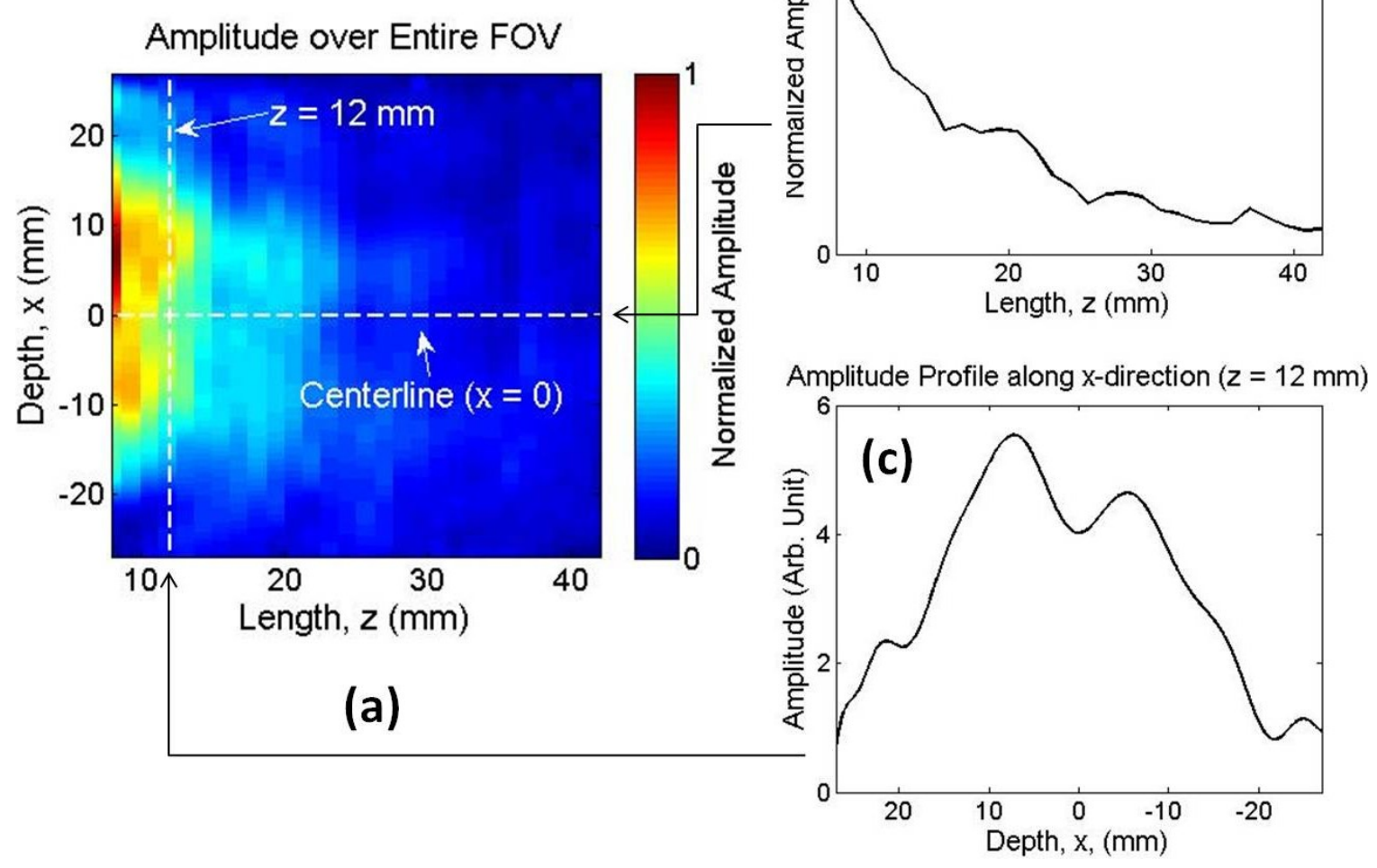

Figure 7.2. An example of measured SW amplitude variations: (a) SW amplitude map over entire FOV, (b) amplitude profile along the centerline, and (c) amplitude profile at $z$ $=12 \mathrm{~mm}$.

$A T T(z)$ in Eq. (7.2) can be numerically calculated by a diffraction theory [40] with known parameters of the measurement configuration as described in Section 7.2.2. Thus, 
the measured $A_{I}(z)$ in a chosen range of $z$ was compared to the $A_{I}(z)$ calculated by $\mathbf{E q}$. (7.2) with an assumed $\alpha_{S}$ value to find the best estimated $\alpha_{S}$ which gave the minimum mean square error between the measured and calculated $A_{I}(z)$.

\subsubsection{Numerical Simulation of Diffraction Effect}

The SW amplitude change with respect to the distance $z$ due to the diffraction can be calculated using a Huygens-Fresnel diffraction theory [40] with the known values of $v_{S}$

and $f_{S}$, and the characteristic size of the SW source $a$ and $b$ which were the half length and width of the square SW source, respectively.

According to the Huygens-Fresnel principle of a rectangular source, when $a$ and $b$ were comparable to the wavelength of the SW applied, the SW was experiencing diffraction. Then the equations of SW $V$ can be expressed as the followings.

$$
\begin{aligned}
& V(x, y, z)=\frac{-1}{4 \pi} \cdot \int_{-a}^{+a} \int_{-b}^{+b} 2 A_{S} \cdot \cos \varphi \cdot\left(i k_{S}-\frac{1}{r_{I}}\right) \cdot \frac{e^{i k_{S} r_{I}}}{r_{I}} d x^{\prime} d y^{\prime} \\
& r_{I}=\sqrt{\left(x-x^{\prime}\right)^{2}+\left(y-y^{\prime}\right)^{2}+z^{2}} \\
& \cos \varphi=\frac{z}{r_{I}}
\end{aligned}
$$

where $r_{I}$ is the traveling distance of the SW from an arbitrary point $\left(x^{\prime}, y^{\prime}, 0\right)$ on the $\mathrm{SW}$ vibration source to an arbitrary point $(x, y, z)$ inside the specimen. $\varphi$ is the angle between $r_{I}$ and $z$-direction. The geometry of such diffraction is demonstrated in Fig. 7.3. 


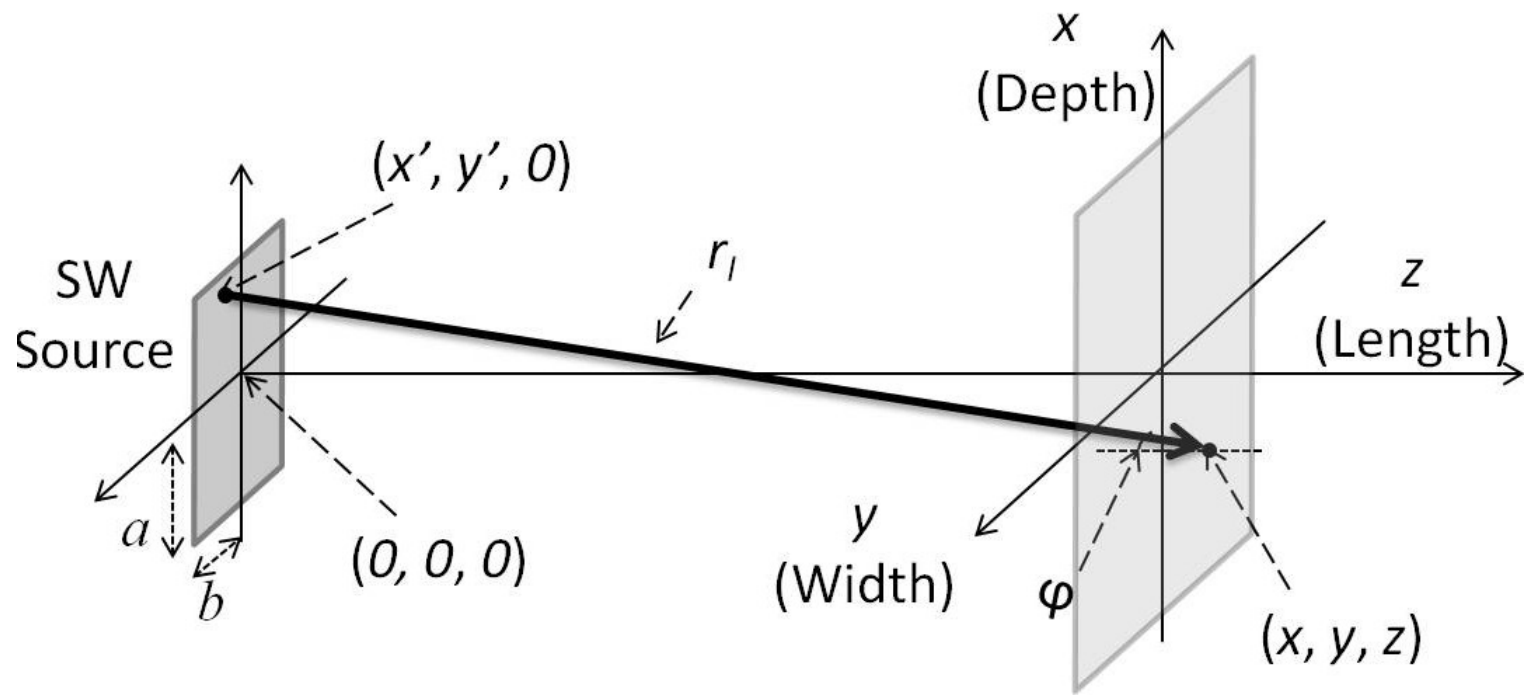

Figure 7.3. The geometry of Fresnel diffraction of a rectangular source with considering the interference between induced and reflected waves.

$V_{I}$ on the $x z$-plane was calculated by Eq. (7.3) using MATLAB. The vibration direction of the SW source is along $x$-direction. The spatial intervals in the vibration source $d x^{\prime}$ and $d y^{\prime}$ were assigned to $100 \mu \mathrm{m}$. Note that simulation with even smaller $d x^{\prime}$ and $d y$ ' values were also conducted, but it did not show noticeable difference. Therefore in order to reduce the time consumption of computation, $d x^{\prime}$ and $d y^{\prime}$ at $100 \mu \mathrm{m}$ were used in the following calculation results.

2D maps of the SW displacement and amplitude at $y=0$ were calculated on semifinite mediums with $\lambda_{S}=10 \mathrm{~mm}\left(f_{S}=500 \mathrm{~Hz}\right.$ and $\left.v_{S}=5 \mathrm{~m} / \mathrm{s}\right)$ and with $a=b=0.5 \mathrm{~mm}$, $2.5 \mathrm{~mm}, 5 \mathrm{~mm}, 10 \mathrm{~mm}, 20 \mathrm{~mm}$, and $40 \mathrm{~mm}$. The calculated results in $x z$-plane were shown in Fig. 7.4. It can be seen that the SW displacements were more close to plane waves as the characteristic size $a$ increases, while the SW displacements were more diverged when $a$ was comparable to and smaller than the $\lambda_{s}$. The SW amplitude changed with $z$ due to the diffraction. 


$$
\begin{aligned}
& \lambda_{S}=10 \mathrm{~mm} \\
& a=0.5 \mathrm{~mm}
\end{aligned}
$$

$\lambda_{S}=10 \mathrm{~mm}$ $a=2.5 \mathrm{~mm}$

$$
\lambda_{S}=10 \mathrm{~mm}
$$$$
a=5 \mathrm{~mm}
$$

$$
\begin{aligned}
& \lambda_{s}=10 \mathrm{~mm} \\
& a=10 \mathrm{~mm}
\end{aligned}
$$

$\lambda_{S}=10 \mathrm{~mm}$ $a=20 \mathrm{~mm}$
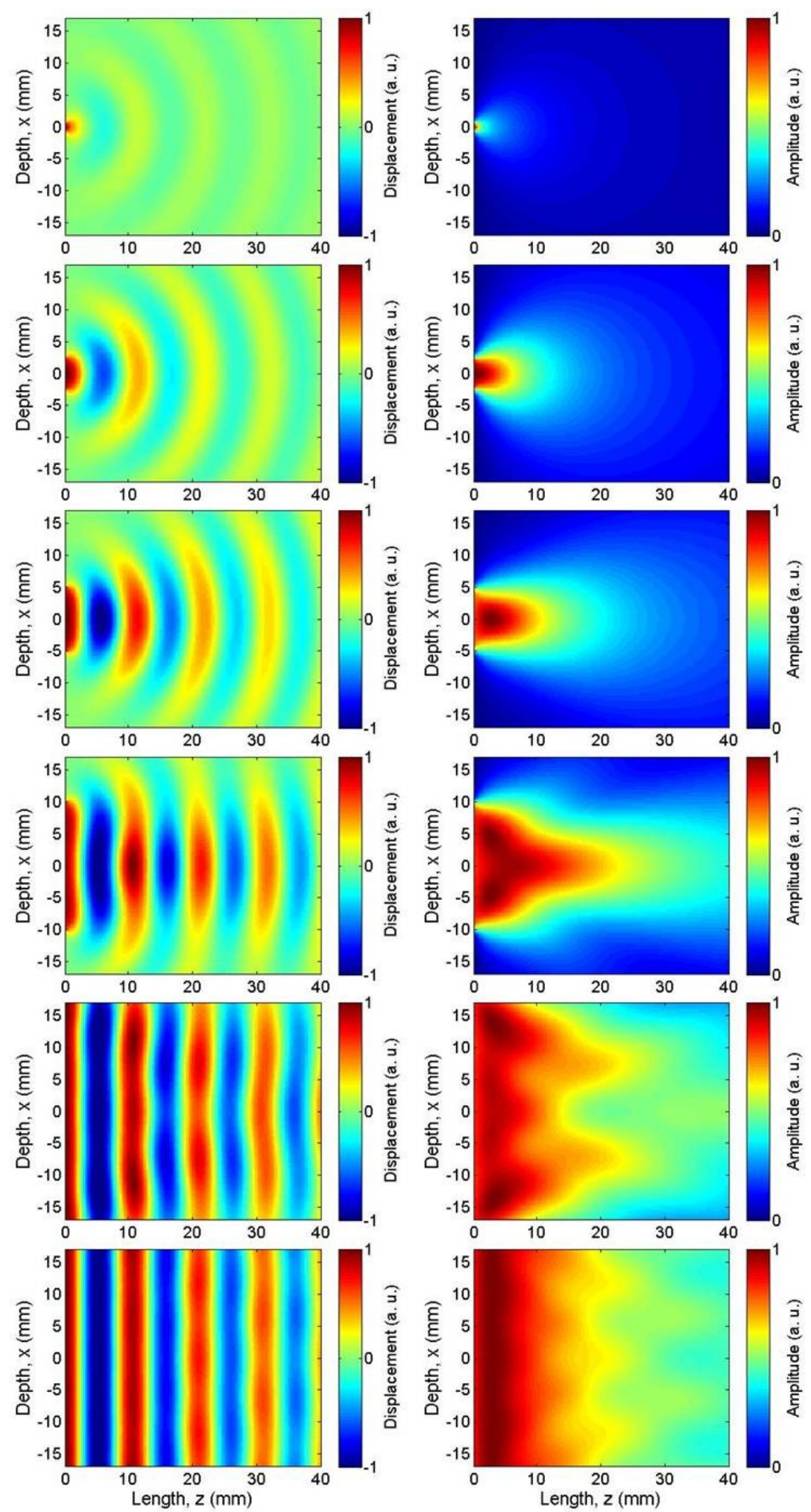

Figure 7.4. The appearance of the SW displacements (left) and amplitudes (right) calculated from different size of the SW sources. The SW wavelength $\left(\lambda_{S}\right)$ was $10 \mathrm{~mm}$, and the characteristic sizes $(a)$ of the SW source were $0.5 \mathrm{~mm}, 2.5 \mathrm{~mm}, 5 \mathrm{~mm}, 10 \mathrm{~mm}, 20$ $\mathrm{mm}$, and $40 \mathrm{~mm}$, from top to bottom. 


\subsubsection{Selection of Absorption Measurement Location}

The SW amplitude change due to diffraction effect depended on $z$ locations where the SW propagated. According to the Fresnel diffraction theory, the SW amplitude change with respect to $z$ caused by the diffraction effect $(\operatorname{ATT}(z))$ was associated with the $k_{S} a$ value (a product of $k_{S}$ and $a$ ) and normalized distance $\mathrm{S}\left(=z / N_{F}\right)$, where $S$ is the normalized distance and $N_{F}$ is the Fresnel distance expressed by Eq. (7.6) [40]. A simulation of the SW amplitude change along the $z$ centerline $(x=0)$ due to the diffraction was conducted with the $k_{S} a$ value of 10 . The result of the amplitude profile was plotted in Fig. 7.5 with respect to a normalized distance $S$.

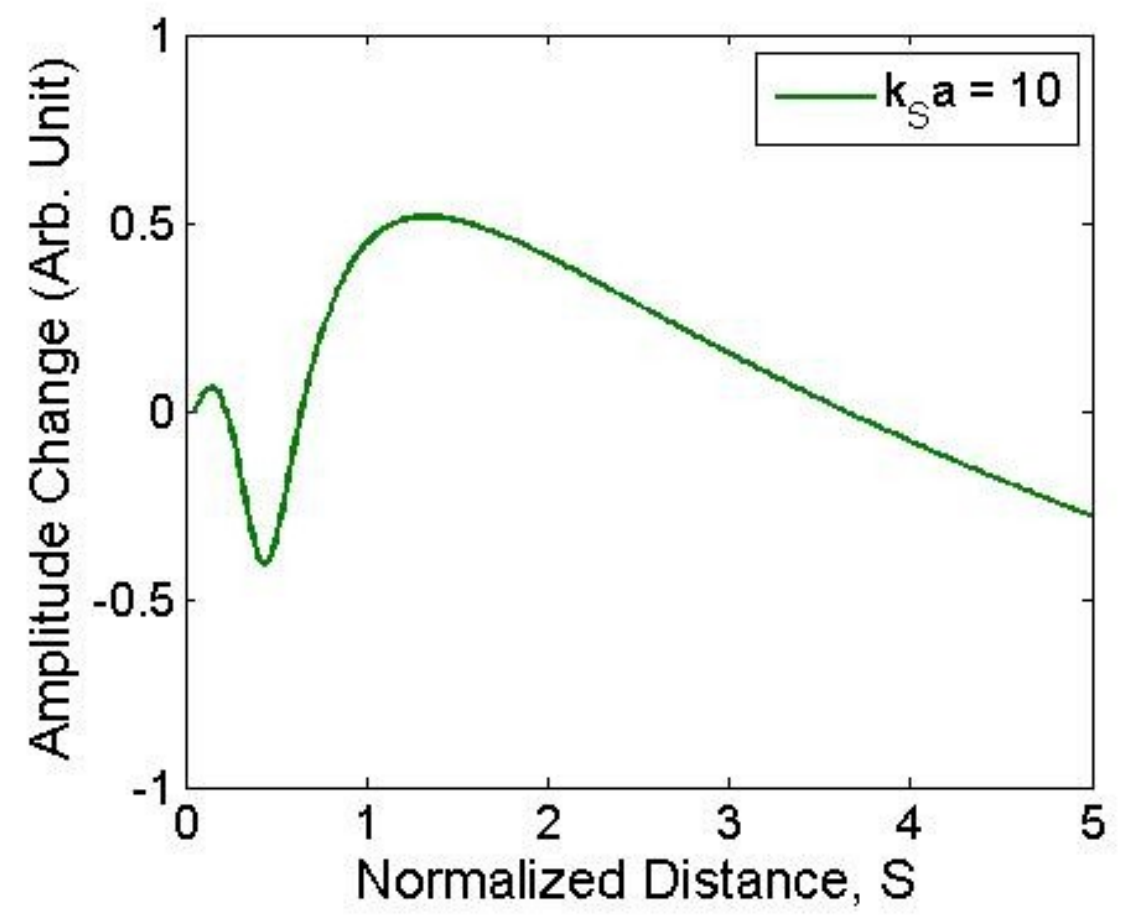

Figure 7.5. A simulation of amplitude change due to diffraction effect on centerline with respect to a normalized distance. The $k_{s} a$ value was 10 .

$$
N_{F}=a^{2} \cdot \frac{f_{S}}{v_{S}} .
$$


It can be seen that the amplitude fluctuates and changed quickly when the normalized distance $S<1$ (near field). It had a peak value at $S=1$ and started to decrease monochromatically after $S=1$. Therefore, the slope of the amplitude change due to diffraction was depending on the $S$.

Figure 7.6 presented $\operatorname{ATT}(z)$ calculated with different $k_{S} a$ values with respect to the normalized distance $S$. $k_{S} a$ values were 2, 5, 10, and 20, and $\alpha_{S}$ was $0 \mathrm{~Np} / \mathrm{m}$ for each calculation.

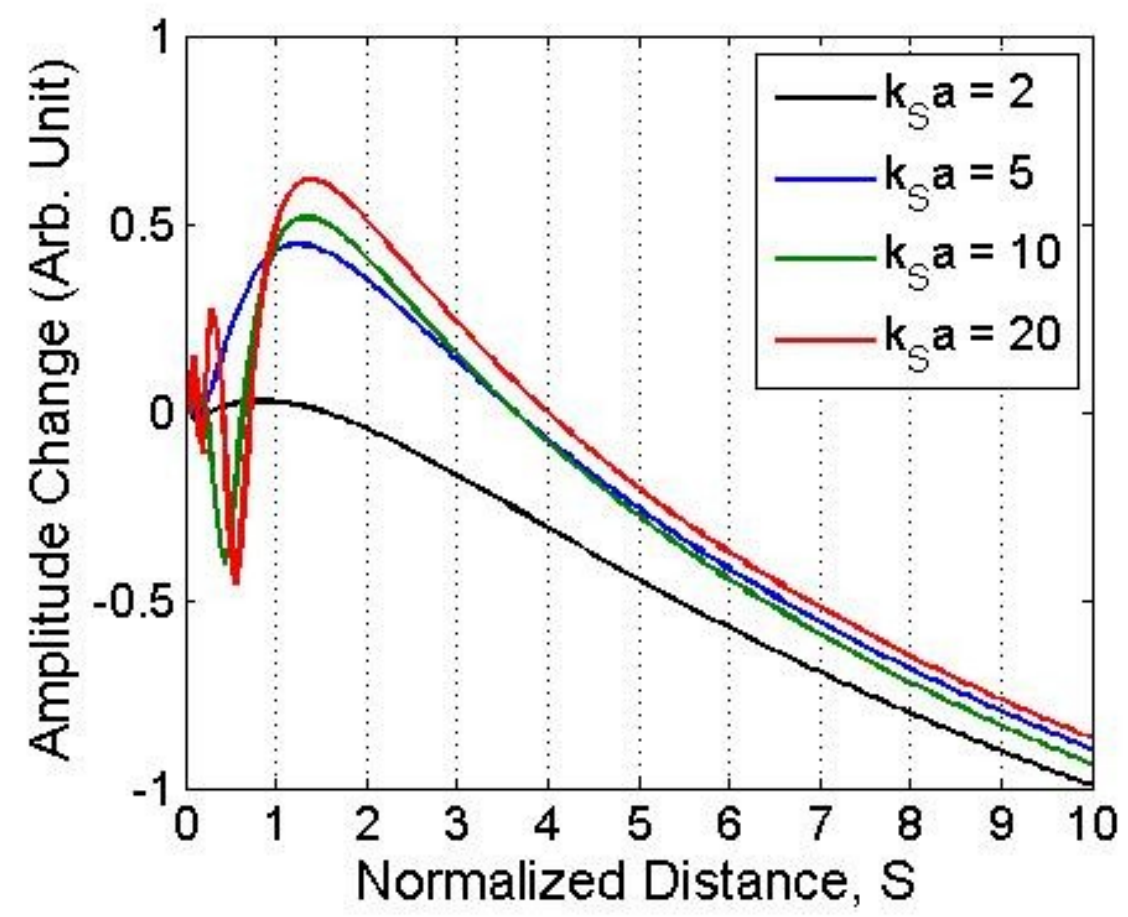

Figure 7.6. Simulations of amplitude change due to diffraction effect on centerline with respect to a normalized distance. Black-line represented $k_{S} a=2$, blue-line represented $k_{S} a$ $=5$, green-line represented $k_{S} a=10$, and red-line represented $k_{S} a=20$.

Since $\alpha_{S}$ was determined by the measured SW amplitude change with the compensation of the diffraction effect, the accurate estimation of $\operatorname{ATT}(z)$ by numerical calculation was crucial. The measurement accuracy of the $z$-range where the SW 
amplitude was measured was depending on the precision of the positioning device employed. Therefore, inaccurate measurement of the $z$-range due to the limitation of the positioning device could cause an error in the $\operatorname{ATT}(z)$ calculation, and thus resulted in $\alpha_{S}$ estimation error. In order to minimize the $A T T(z)$ calculation error due to the positioning error of $z$-range of the SW amplitude measurement, $z$-range where the $\operatorname{ATT}(z)$ change was less sensitive to $z$ was investigated through numerical simulations as follows.

The factor of positioning error of $z$-range was added into the simulations as follows. Assuming that the coverage of $S$-range was 5 , the ATT was calculated in five different $S$-ranges for each $k_{S} a$ value as follows: $S=[0,5], S=[1,6], S=[2,7], S=[3,8]$, and $S=[4,9]$. It was assumed that the positioning error of the $S$-range was $S+0.5$ or $S-$ 0.5 as listed in Table 7.1. For instance, in region \#2 of the simulation with $k_{S} a=10$, an $A_{B}(S)$ that calculated in $S=[1,6]$ was mistakenly recorded at $S=[0.5,5.5]$ or $S=[1.5$, 6.5], and it was termed $A_{B_{-} m r}(S \pm 0.5)$. Such example was shown in Fig. 7.7. It was noted that the positioning error of $S-0.5$ was not applicable in $\# 1(S=[0,5])$.

Table 7.1. Five regions in simulations to investigate the error due to positioning error.

\begin{tabular}{|c|c|c|}
\hline Region \# & S-range & Positioning Error on $\boldsymbol{S}$ location \\
\hline$\# 1$ & $0-5$ & +0.5 \\
\hline$\# 2$ & $1-6$ & \pm 0.5 \\
\hline$\# 3$ & $2-7$ & \\
\hline$\# 4$ & $3-8$ & \\
\hline$\# 5$ & $4-9$ & \\
\hline
\end{tabular}


Figure 7.7 showed the calculation results of SW amplitude change due to diffraction with $k_{S} a=10$. True $S$-range was $[1,6]$ (top), positioning error of $S$ values with -0.5 was $[0.5,5.5]$ (middle), and that with +0.5 was $[1.5,6.5]$ (bottom).

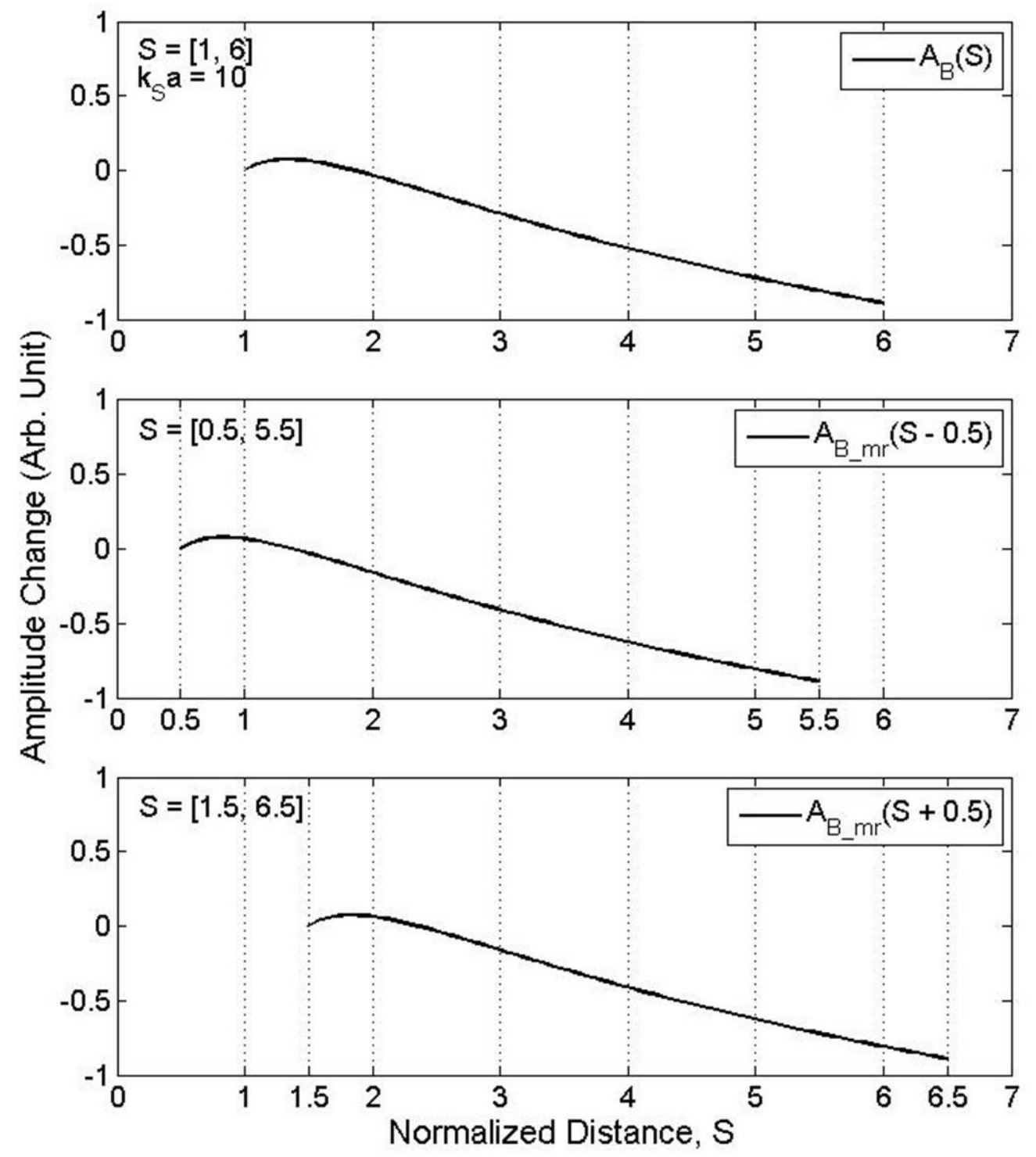

Figure 7.7. Simulation of SW amplitude change due to diffraction with $k_{S} a=10$, the actual $S$-range was $[1,6]$ (top), and was mistakenly recorded to $S=[0.5,5.5]$ (middle), and $S=[1.5,6.5]$ (bottom).

Estimation error of $\alpha_{S}$ due to the positioning error of $S$-range was investigated using the calculation results in Fig. 7.7 as follows. A least square curve fitting technique 
was used to find of the best $\alpha_{S}$ value that yielded an $A_{B}{ }^{\prime}(S \pm 0.5)$ which best fitted the profile of $A_{B_{-} m r}(S \pm 0.5)$. Since the true $\alpha_{S}$ value was zero for the calculated SW amplitude changes in Fig. 7.7, estimated value of $\alpha_{S}$ was considered as the calculation error due to the positioning error. The curve fitting resulted $A_{B}{ }^{\prime}(S \pm 0.5)$ for $A_{B_{-} m r}(S \pm 0.5)$ in Fig. 7.7 were demonstrated by the dot-lines in Fig. $\mathbf{7 . 8}$.

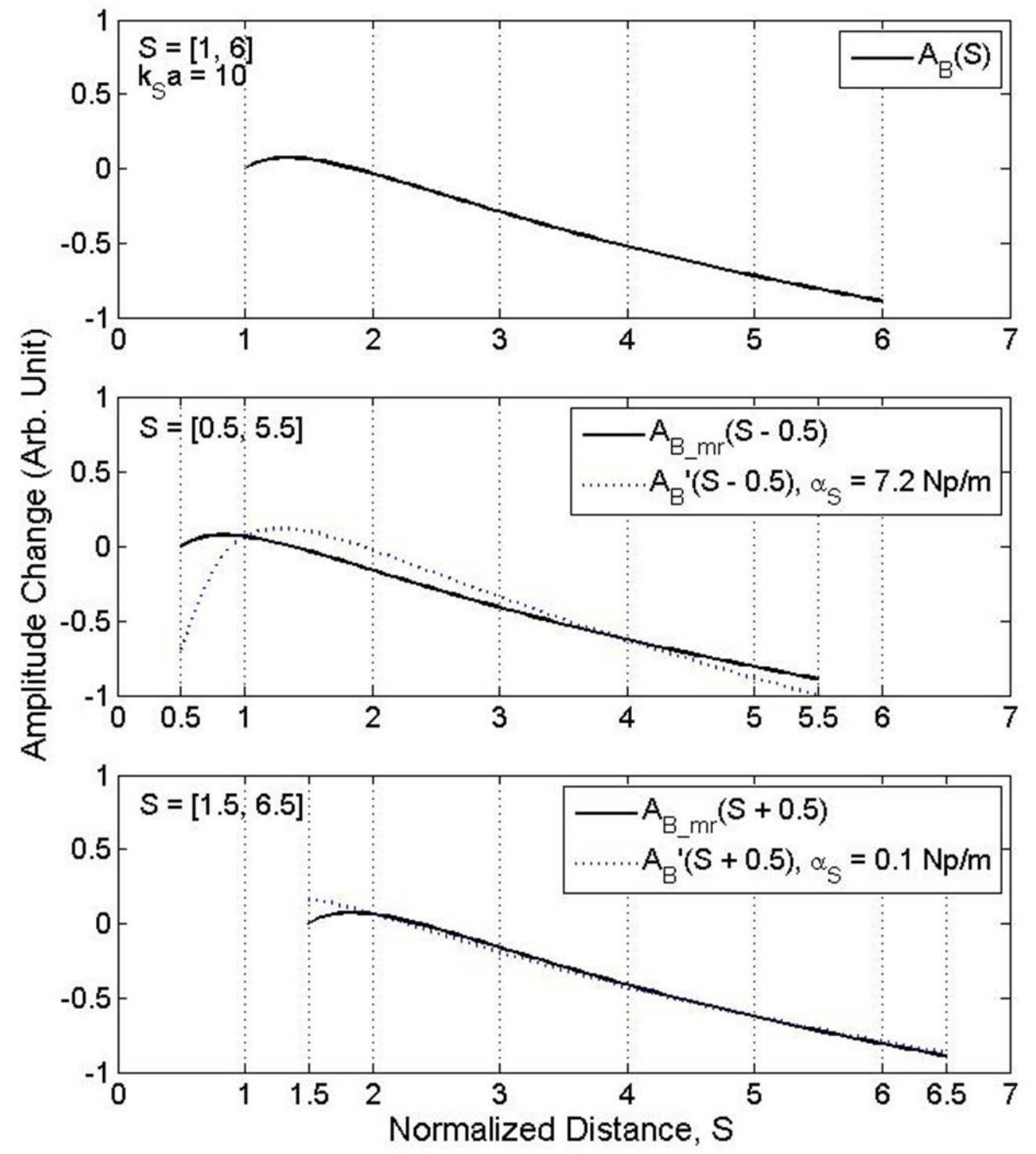

Figure 7.8. Curve fitting results (indicated by dot-lines) of the SW amplitude with positioning error (indicated by solid-lines) in Fig. 7.7. 
Figure 7.9 showed the estimation errors of $\alpha_{S}$ caused by the positioning error for different $k_{S} a$ values. All the $k_{S} a$ values examined had the greatest error at \#1 $S$-range (where only had a positioning error of +0.5 ). The smallest $\alpha_{S}$ estimation error was expected at the $S$-range $[2,7]$ for $k_{S} a=2$, and after $[2,7]$ for other $k_{S} a$ values. It can be seen that the $\alpha_{S}$ estimation error was greater in the Fresnel field $(S<1)$, thus the effect of the positioning error was relatively great if the location of the measurement started from $S=0$. It can be also seen that the error could be even smaller with the $S$-range far away from the SW source. However, the strength and SNR of the SW signals decreased when the $S$-range in the SW amplitude measurement was away from the SW source in practice.
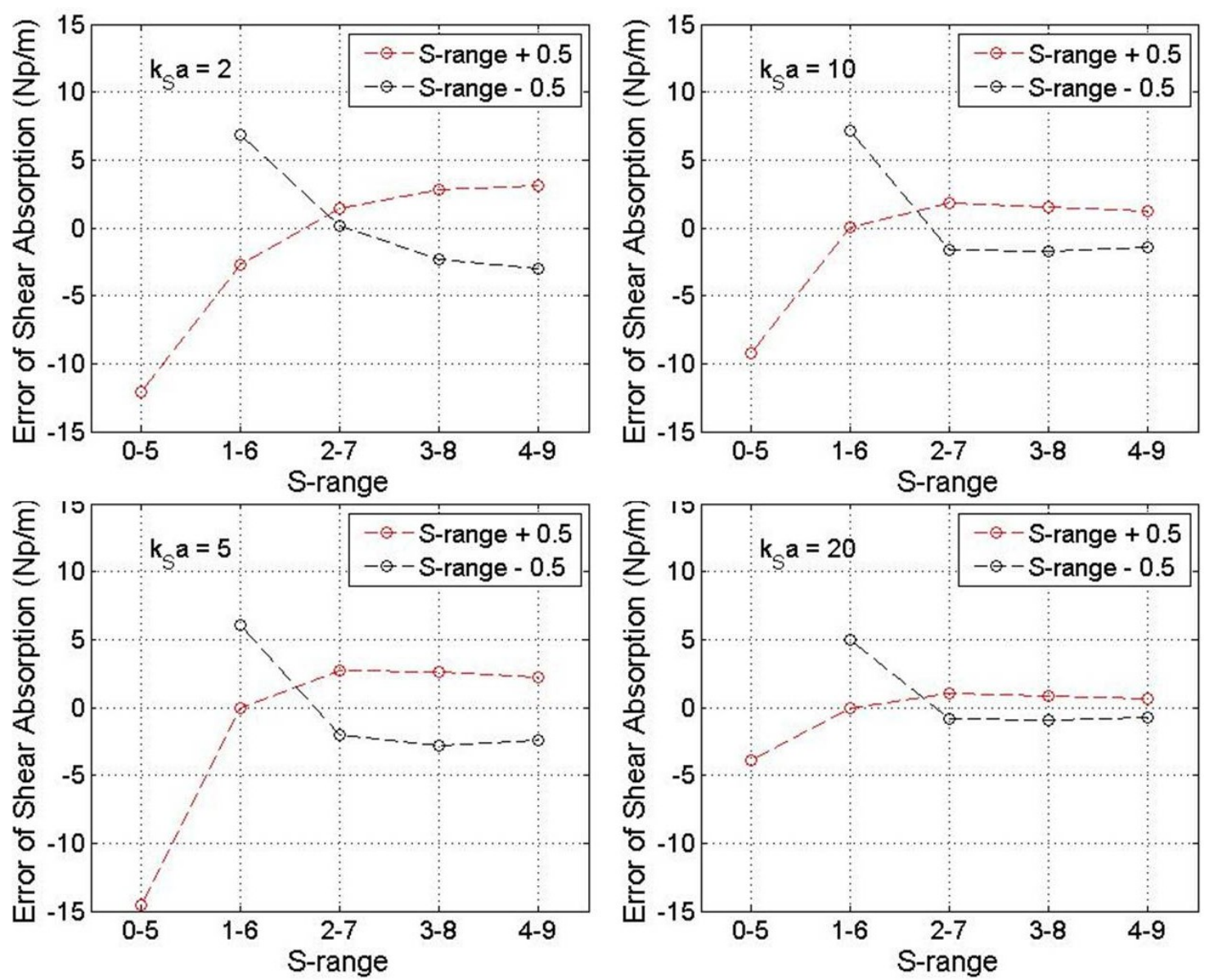

Figure 7.9. Calculation error on the amplitude change of diffraction effect due to positioning error. Red-circle dash line represented the positioning error for $S+0.5$, and blue-circle dash-line represented the positioning error for $S-0.5$. 


\subsection{Experiment}

\subsubsection{Experimental Setup}

A homogeneous hydrogel phantom was fabricated for the experiment to verify the proposed method. The phantom had a cubic shape with a side length of $50 \mathrm{~mm}$. It was made of $9-\mathrm{W} \%$ gelatin and $2-\mathrm{W} \%$ graphite. The averaged $v_{S}$ of the phantom was measured $2.15 \mathrm{~m} / \mathrm{s}$ by the proposed $v_{S}$ measurement method developed in Chapter 4 . The $v_{S}$ image of the phantom was shown in Fig. 7.10.

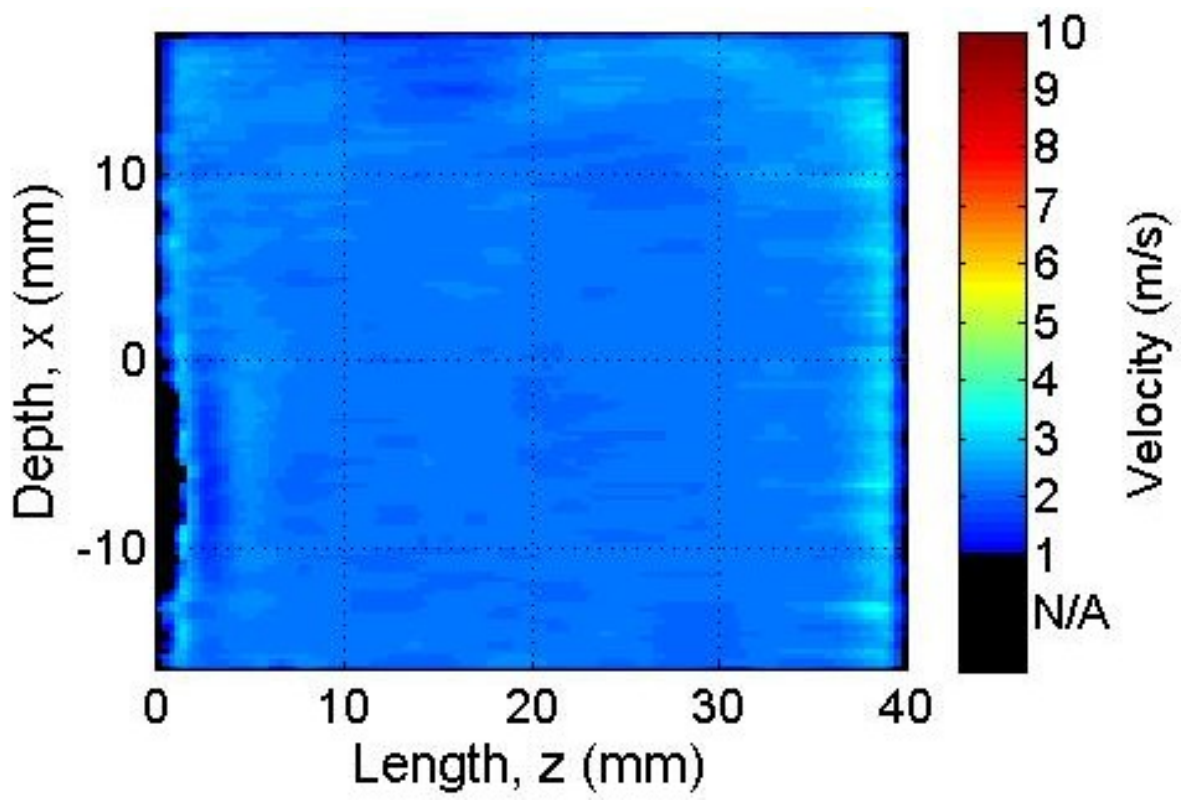

Figure 7.10. SW velocity image of the gelatin phantom.

In this experiment, the $f_{S}$ was $227 \mathrm{~Hz}$, and the $a$ of the square $\mathrm{SW}$ source was 5 $\mathrm{mm}$, so the $k_{S} a$ value was calculated 3.32. The amplitude change due to diffraction with this $k_{S} a$ value was calculated and plotted in Fig. 7.11. The error on $\alpha_{S}$ measurement due to positioning error was investigated according to the method described in Section 7.2.3. Twenty one (21) ranges in $z$-direction (z-range) were defined and listed in Table 7.2. The 
coverage of each $z$-range was $30 \mathrm{~mm} . \pm 1 \mathrm{~mm}$ positioning error (except the first $z$-range, which only had $+1 \mathrm{~mm}$ positioning error) was assumed for each $z$-range. Fig. 7.12 showed the estimation error of $\alpha_{S}$ for each $z$-range.

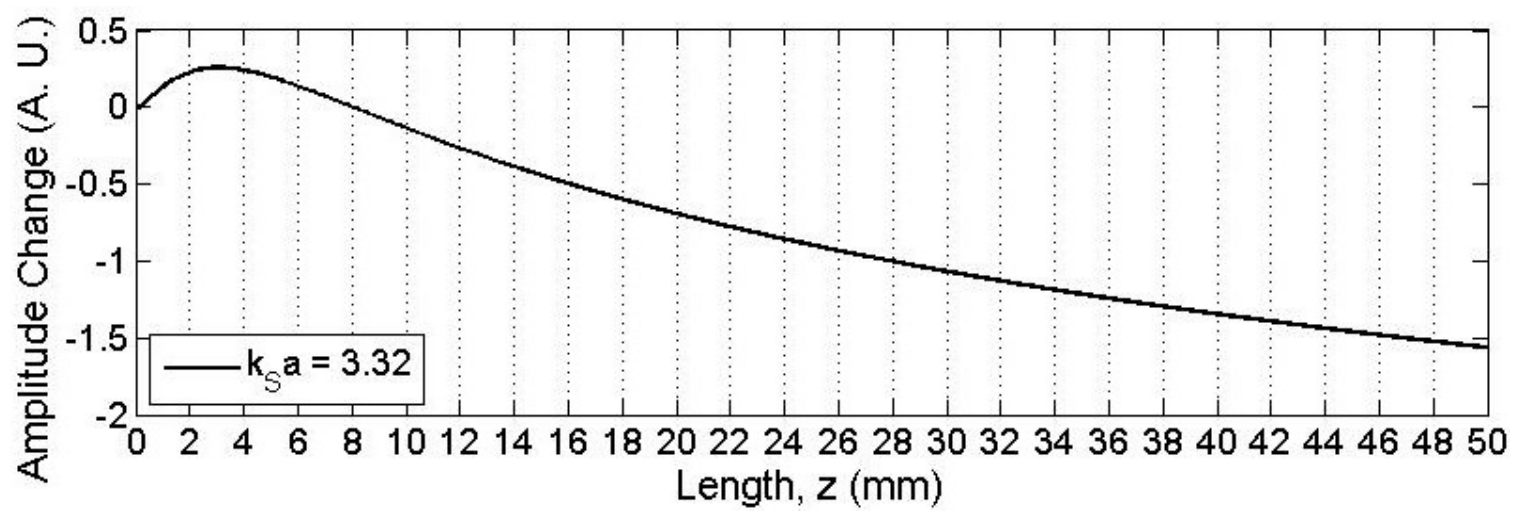

Figure 7.11. A simulation of amplitude change due to diffraction effect on centerline with respect to the length $z$. The $k_{S} a$ value was 3.32 .

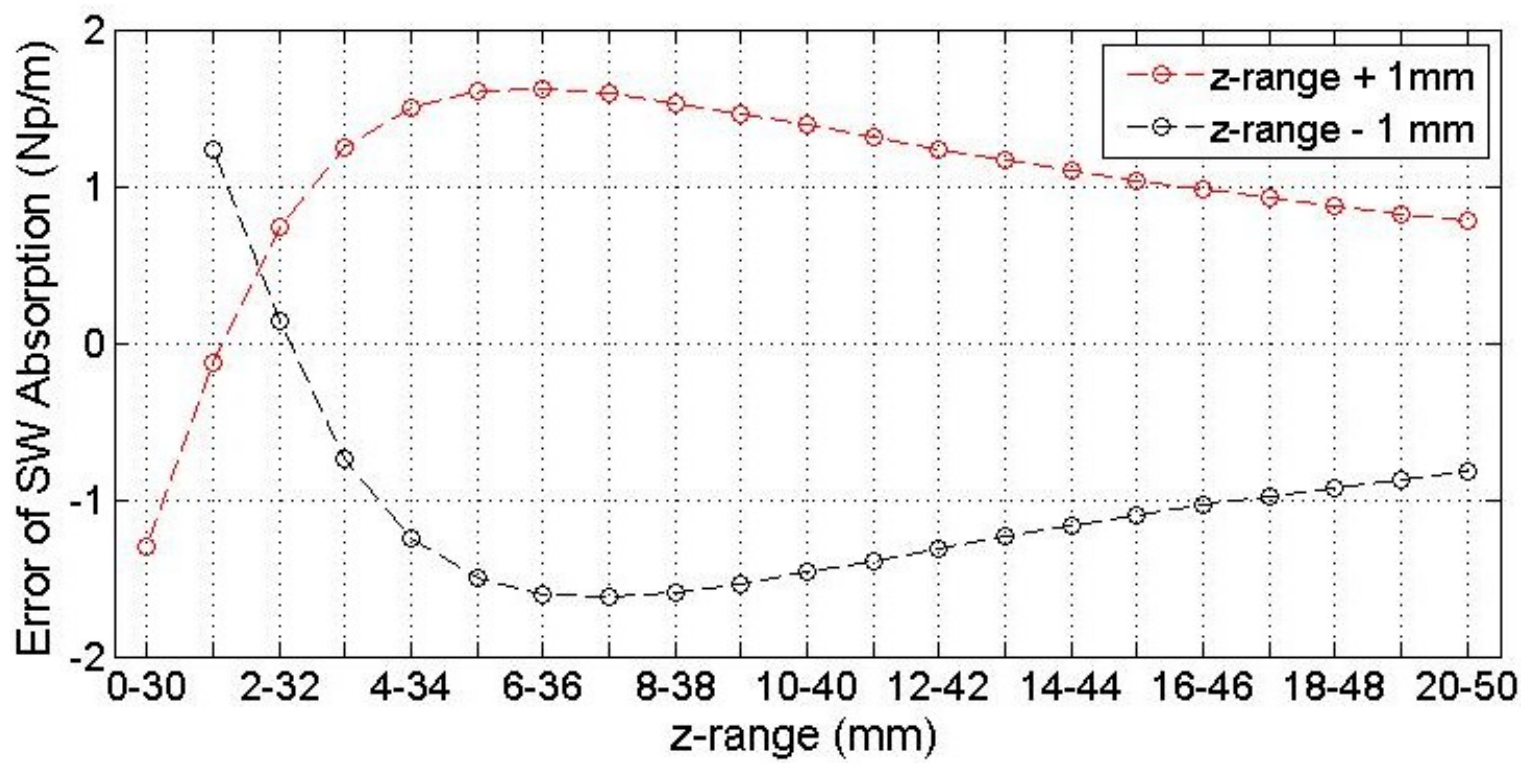

Figure 7.12. Estimation error of SW absorption due to the positioning error of $z$-location for $k_{S} a=3.32$. Red-circle dash-line represented the positioning error of $z+1 \mathrm{~mm}$, and blue-circle dash-line represented the positioning error of $z-1 \mathrm{~mm}$. 
Table 7.2. Twenty one regions in phantom experiment to investigate the error due to positioning error.

\begin{tabular}{|c|c|c|}
\hline Region \# & $z$-range $(\mathrm{mm})$ & Positioning Error on $z$ location $(\mathrm{mm})$ \\
\hline$\# 1$ & $0-30$ & +1 \\
\hline$\# 2$ & $1-31$ & \multirow{20}{*}{ \pm 1} \\
\hline \#3 & $2-32$ & \\
\hline$\# 4$ & $3-33$ & \\
\hline$\# 5$ & $4-34$ & \\
\hline \#6 & $5-35$ & \\
\hline$\# 7$ & $6-36$ & \\
\hline$\# 8$ & $7-37$ & \\
\hline$\# 9$ & $8-38$ & \\
\hline$\# 10$ & $9-39$ & \\
\hline$\# 11$ & $10-40$ & \\
\hline \#12 & $11-41$ & \\
\hline$\# 13$ & $12-42$ & \\
\hline$\# 14$ & $13-43$ & \\
\hline \#15 & $14-44$ & \\
\hline$\# 16$ & $15-45$ & \\
\hline \#17 & $16-46$ & \\
\hline$\# 18$ & $17-47$ & \\
\hline \#19 & $18-48$ & \\
\hline \#20 & $19-49$ & \\
\hline$\# 21$ & $20-50$ & \\
\hline
\end{tabular}


From Fig. 7.12 it can be seen that the error on the $\alpha_{S}$ measurement due to the positioning error reached the minimum values at $z=1$ to $31 \mathrm{~mm}(S=0.38$ to 11.7$)$, for $z$ $+1 \mathrm{~mm}$ (red-circle dash-line). The minimum error for $z-1 \mathrm{~mm}$ (blue-circle dash-line) was found at $z=2$ to $32 \mathrm{~mm}(S=0.76$ to 12.1$)$, where such $z$-range also resulted in the second minimal error values for $z+1 \mathrm{~mm}$. Therefore, $z$-range of 2 to $32 \mathrm{~mm}$ was used in the measurement of the $\alpha_{S}$ presented in the next section.

\subsubsection{Measurement of Shear Wave Absorption}

The experimental configuration of the $\alpha_{S}$ measurement was depicted in Fig. 7.13. The SW source (square Plexiglas plate) had a $10 \mathrm{~mm}$ side length $(a=b=5 \mathrm{~mm})$. The SW source was continuously driven by $227 \mathrm{~Hz}$ during the measurement. The setup of the ultrasound system was the same as that used for the $v_{S}$ measurements. According to the above discussion, the field-of-view (FOV) in $z$-direction $\left(\mathrm{FOV}_{\mathrm{z}}\right)$ in this measurement was 30-mm and the $z$-range was selected at $z=2$ to $32 \mathrm{~mm}$. 


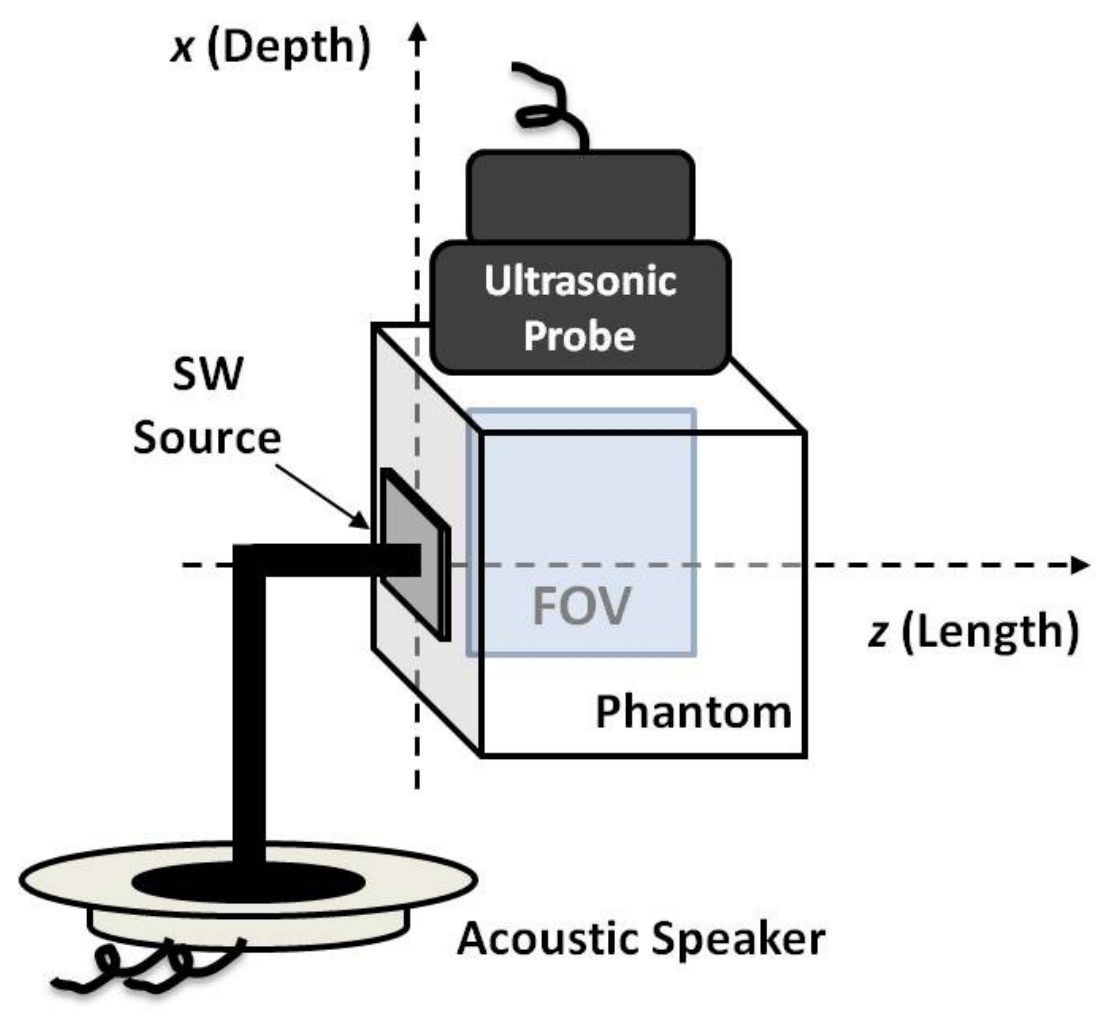

Figure 7.13. Experimental configuration of SW absorption measurement.

\subsection{Results and Discussion}

A chosen frame of the induced SW over the FOV was presented in Fig. 7.14(a). SW amplitude $A_{I}$ at each frame was calculated by Eq. (7.2) and then averaged with 180 frames. The result of $A_{I}$ was shown in Fig. 7.14(b) to be used to find the centerline $(x=0)$, as indicated by the dash-line. The $A_{I}$ on the centerline was shown in Fig. 7.14(c) as the blue-dot. 

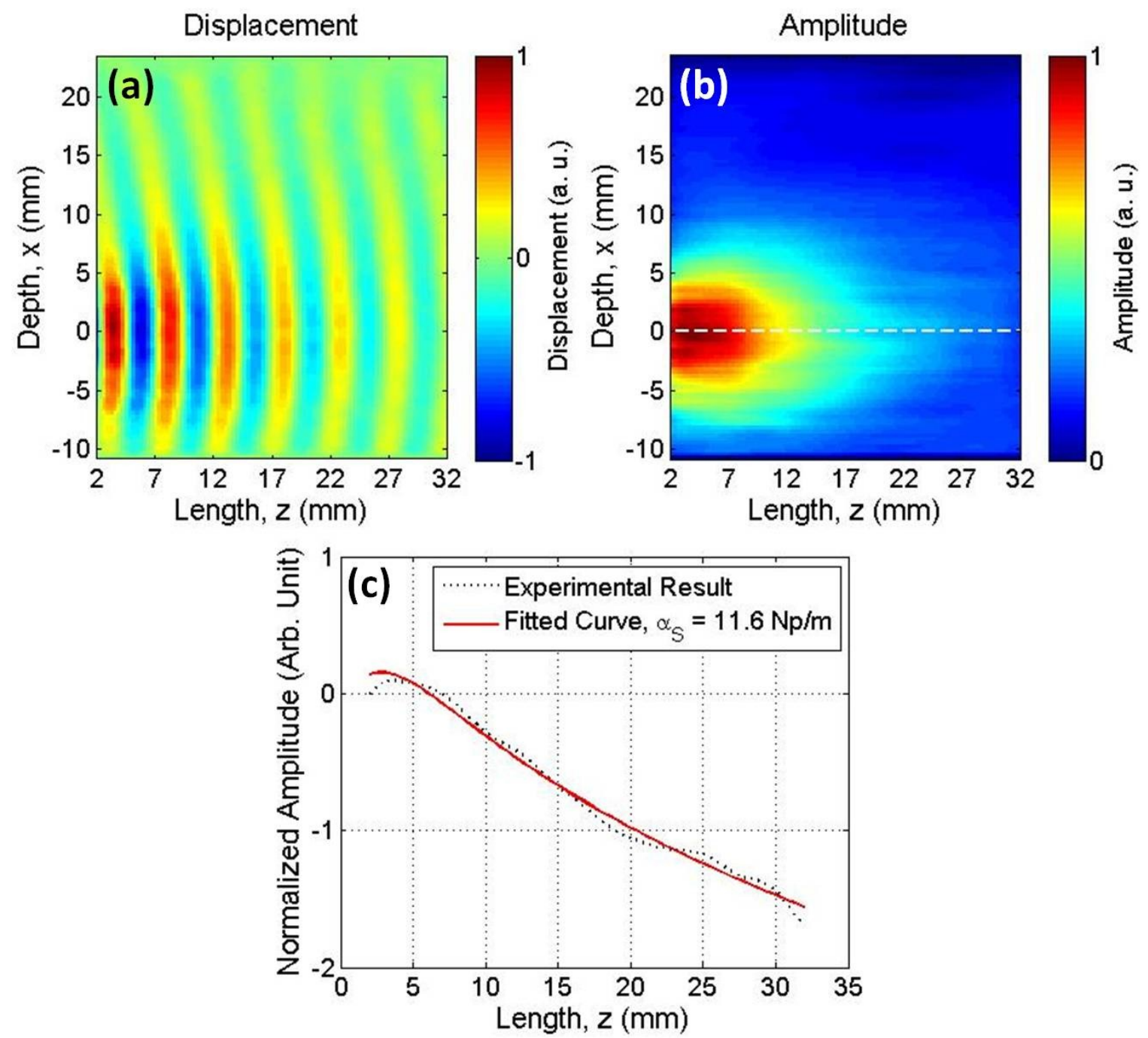

Figure 7.14. The experimental result of (a) induced SW displacement $\left(V_{I}\right)$, (b) SW amplitude $\left(A_{I}\right)$, and (c) the SW amplitude on the centerline $(x=0)$ in (b).

By using the least square curve fitting technique, the calculated best fit curve (redsolid line) to the experimental result (blue-dot line) was obtained with $\alpha_{S}=11.6 \mathrm{~Np} / \mathrm{m}$ in Eq. (7.2).

A simulation of the $A_{I}$ was calculated by using $v_{S}=2.15 \mathrm{~m} / \mathrm{s}, \alpha_{S}=11.6 \mathrm{~Np} / \mathrm{m}$, and $f_{S}=227 \mathrm{~Hz}$ in Eq. (7.2). Fig. 7.15(a) showed the simulation result over the entire FOV 
comparing with the experimental result in Fig. 7.15(b) under the same amplitude scale. The simulation result showed a good correlation to the experimental result.

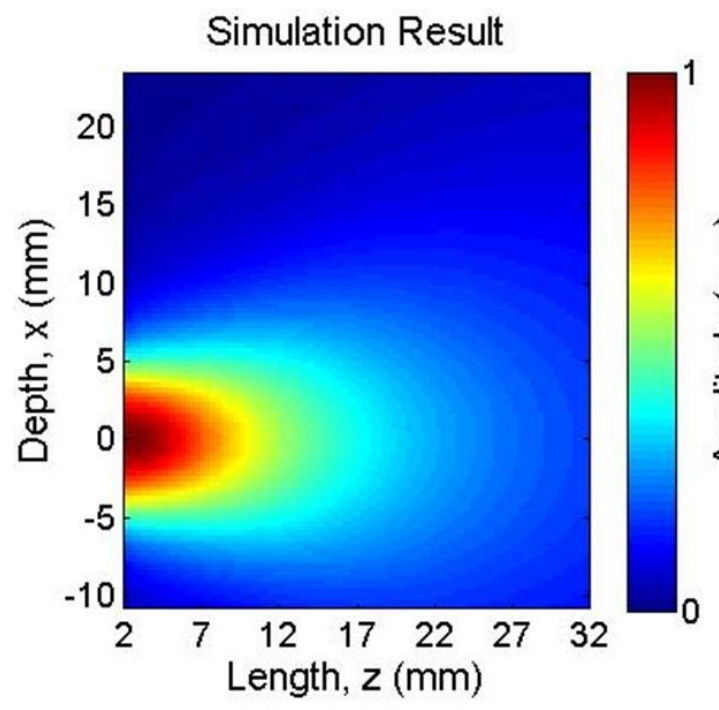

(a)

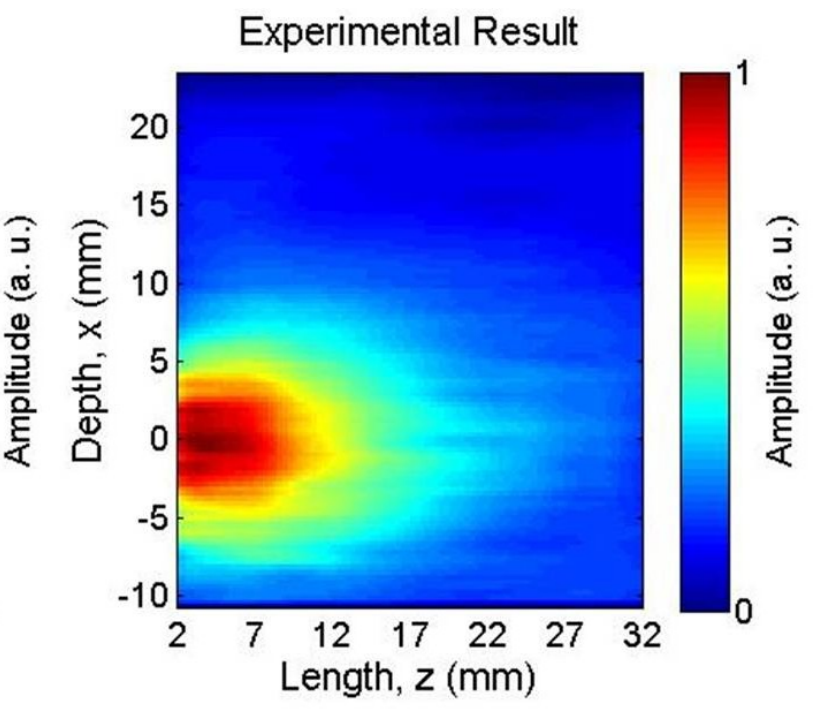

(b)

Figure 7.15. Comparison between the simulation result (a) and experimental result (b) of the SW amplitude.

Finally, the shear elasticity $G$ and viscosity $\eta$ of the phantom were calculated by using Eqs. (2.6) and (2.7). The $G$ was calculated $5.06 \mathrm{kPa}$, and the $\eta$ was calculated 0.12 $\mathrm{Pa} \cdot \mathrm{s}$.

Standard tissue-mimicking phantoms were available commercially for the calibration of ultrasound system (i.e. the actual longitudinal parameters, such as LW velocity and attenuation were known in those phantoms) [110]. However, after a careful literature research, as far as the author was concerned, no commercial phantom was available specifically for the measurement of viscoelasticity. In most cases the researchers would have to fabricate soft tissue-mimicking phantoms by themselves for individual experiment to reach their experimental desires. As a result, the actual shear 
parameters, such as $v_{S}$ and $\alpha_{I}$ would be unknown for those phantoms [93], [105], [110][112]. Currently, the most promising method to verify the viscoelastic properties of a sample tissue could be the supersonic shear imaging (SSI) [16], yet such method was not available in our measurement system. Therefore, the calculated $G$ and $\eta$ in this study were only compared with the published results using similar phantom specimens, as listed in the Table 7.3.

Table 7.3. A comparison between our result and published results using similar phantom specimens.

\begin{tabular}{|c|c|c|c|c|}
\hline $\begin{array}{l}\text { Measurement } \\
\text { Method }\end{array}$ & Phantom & $\begin{array}{c}\text { Shear } \\
\text { Modulus } \\
\text { G (kPa) }\end{array}$ & $\begin{array}{c}\text { Shear } \\
\text { Viscosity } \\
\eta(\mathbf{P a} \cdot \mathbf{s})\end{array}$ & Reference \\
\hline SSI & $\begin{array}{c}\text { Gelatin }+ \text { agar, } \\
\text { percentage was } \\
\text { not specified }\end{array}$ & 4 & 0.3 & {$[15]$} \\
\hline SDUV & $\begin{array}{c}\text { 10-W\% Pigskin } \\
\text { powder }+20-\mathrm{W} \% \\
\text { Castor oil }\end{array}$ & 4.49 & 2.41 & [105] \\
\hline \multirow{2}{*}{ SDUV } & $\begin{array}{c}12-\mathrm{V} \% \text { "Bloom } \\
75 " \text { gelatin }\end{array}$ & 2.22 & 0.24 & \multirow{2}{*}[17]{} \\
\hline & $\begin{array}{l}12-\mathrm{V} \% \text { "Bloom } \\
300 " \text { gelatin }\end{array}$ & 5.36 & 0.38 & \\
\hline \multirow{2}{*}{ SWEI } & $3-\mathrm{W} \%$ gelatin & 2.01 & 0.6 & \multirow{2}{*}{ [112] } \\
\hline & $6-\mathrm{W} \%$ gelatin & 8.05 & 1.14 & \\
\hline Crawling Wave & $\begin{array}{c}\text { Gelatin phantom, } \\
\text { percentage was } \\
\text { not specified }\end{array}$ & 8.61 & 2.98 & {$[101]$} \\
\hline Our Method & $9-\mathrm{W} \%$ gelatin & 5.06 & 0.12 & N/A \\
\hline
\end{tabular}


The published results had a relatively great variance of the $\eta$ (about 10 times difference between the minimum 0.24 Pa.s and the maximum 2.98 Pa's in the Table 7.3), yet the variance of the $G$ was relatively less (about 4 times difference between the minimum $2.01 \mathrm{kPa}$ and the maximum $8.61 \mathrm{kPa}$ ). Although the viscosity result in our method was less than the published results, it was still in the same order of magnitude of the listed references and it was a reasonable value by considering that the water-based phantom could be a less viscous material [105]. Fabrication and study on the viscous phantom could be good topic for future research.

\subsection{Summary}

This chapter aimed to address the problem due to diffraction effect during the direct measurement of the SW absorption. The SW amplitude was measured by using the same experimental configuration as that for the measurement of SW velocity. The diffraction effect was mathematically calculated by knowing the characteristic size of SW source, SW velocity, and SW frequency, and then it was compensated from the measured SW attenuation. The calculation error during the compensation of the diffraction effect could arise from the positioning error, and such error can be reduced by arranging the measurement onto a spatial region that was less sensitive to the positioning error. Finally the shear elasticity and the viscosity of a specimen were estimated together from the same SW measurement result. Objective 4 in Section 1.3 was achieved by the accomplishment of the research in this chapter. It should be noted that such research was preliminary 
compared with the research on the measurement of SW velocity as presented through

\section{Chapters 3-6.}

It should be also noted that as the SW absorption measured by the method in this chapter was only an averaged value of the entire specimen. Therefore, the estimated shear elasticity and viscosity values also represented the overall viscoelastic properties of the specimen. Nevertheless, the proposed measurement method of SW absorption could still be useful as it can be used to provide reference parameters of viscoelasticity for phantom study and/or in-vitro tissue study such as biopsies. 


\section{Chapter 8}

\section{Conclusions}

\subsection{Summary of Thesis Research}

SW measurement methods for soft tissue characterization were developed with the use of conventional B-mode scan of focused ultrasound and the use of a single external SW source. The proposed methods could reduce the cost and the complexity of overall experimental configuration. A successful measurement of $v_{S}$ and $\alpha_{S}$ would make a proper determination of the tissue shear elasticity and viscosity. Three practical challenges in SW measurement configuration used in this research: 1) Undesired SW reflection, 2) motion artifact, and 3) diffraction effect were studied and addressed as summarized below.

\section{1) Undesired SW reflection from tissue boundary}

When measuring the spatial SW displacement in some experiments, the continuously-induced SW were reflected at the tissue boundary, and interfered with itself. This could cause the deformation of observed SW waveform pattern, and result in errors in the measurement of SW. It was found that the B-mode scanning time delay of a focused ultrasound made the spatial frequencies of the induced and reflected SWs different. Therefore, the undesired SW reflection was 
able to be removed by using a spatial frequency filter on the observed interfered SW displacement. This problem had been addressed in Chapter 3 and Chapter 4.

\section{2) Motion artifact on the measurement of shear wave displacement}

The tissue displacement in a practical SW measurement could include both desired SW displacement and motion artifacts. The undesired motions from the experimental environment and involuntary in-vivo activities may be unavoidable, and resulted in errors in the measurement of SW. In order to overcome this problem, the SW frequency was assigned to a specific frequency which was different from the frequencies of the predictable motion artifacts. This allowed the application of a temporal bandpass filter to reduce the motion artifacts from the measured tissue displacement. This problem had been discussed and addressed in

\section{Chapter 5.}

\section{3) Diffraction effect on the measurement of shear wave absorption}

When the wavelength of a SW was comparable to or larger than the size of the SW source, the diffraction effect must be considered. In order to estimate $\alpha_{S}$ from the measured SW attenuation, the attenuation due to diffraction should be compensated mathematically. The diffraction effect was nonlinear and locationdependent. Therefore, the calculation error during the compensation of the diffraction effect could arise from the inaccuracy of the measurement location. Such error can be reduced by arranging the measurement onto a spatial region that was less sensitive to the positioning error. This problem had been discussed and addressed in Chapter 7. 


\subsection{Scope and Limitation}

The measurement methods of $v_{S}$ and motion artifact reduction were conducted with the phantom specimen and in-vivo tissues. The experimental results positively verified the reliability and usefulness of the proposed measurement methods. The $v_{S}$ is related to the tissue stiffness which may reflect the tissue health. The proposed method of motion artifact reduction was capable of addressing the practical problem of undesired tissue motions during the measurements. Therefore, the proposed measurement method of $v_{S}$ has good potential to be applied for clinical applications such as the diagnosis of the organic lesions, and/or the evaluation of the muscle strength in sports medicine.

The research on the measurement of $\alpha_{S}$ in this thesis was still preliminary. Nevertheless, the shear elasticity and the viscosity of a specimen were estimated together from the same SW measurement result. At current stage, the proposed $\alpha_{S}$ measurement method could be used to provide reference parameters of viscoelasticity for phantom study and/or in-vitro tissue study such as biopsies.

\subsection{Future Research}

The following subjects were not the focus of this thesis, yet they could also be useful for the research on tissue characterization. Therefore, they were listed here and could be good potential research topics in the future.

1) Measureable range of shear wave velocity and B-mode scanning speed

From the study in Chapter 4 it was found that the estimation of $v_{S}$ was depending on the $v_{\text {scan }}$ value of the ultrasound system. Therefore, a change of the $v_{\text {scan }}$ value 
could result in variations of the measureable range and possibly the measurement accuracy of the $v_{S}$. A study on the relationship between the measureable range of $v_{S}$ and the $v_{\text {scan }}$ could be interest and useful if the system allows user to change the $v_{\text {scan }}$.

\section{2) Discrimination of unreliable $S W$ signals}

In practical experiments, the SNR of induced SW may not be known, while weak SW signals (poor SNR) could result in more error. Therefore, method to discriminate the estimated $v_{S}$ from the unreliable SW signals would be helpful to make the $v_{S}$ result more trustworthy. The relevant study is recommended in the future research.

\section{3) Improvement of filtering technique}

In this thesis, a spatial frequency filter was designed and used to remove the unwanted reflected SW. The transition zones in the frequency domain of the filter could undesirably attenuate/remove the wanted induced SW and result in error on the $v_{S}$ measurement result (as discussed in Section $\mathbf{6 . 3}$ for the in-vivo experiment). A frequency-domain filtering technique may help to avoid such concern [113][116]. With this filtering technique, a rectangular mask was directly applied on the spatial frequency domain to retain the wanted frequency component and made unwanted ones zero. Thus, the transition zones would not exist. Preliminary study on such filtering technique had been conducted and demonstrated in Appendix D. Further study is recommended in the future research. 


\section{4) Phantom Fabrication}

Most of the phantoms prepared for this thesis research were dominated by their elastic properties. It was challenging to fabricate a phantom that had both remarkable shear elasticity and viscosity [68], [69], [93], [102], [105], [112], [117], [118]. In addition, the reproducibility of the phantom fabrication was limited. Phantoms with the same compositions but were fabricated at different times could occasionally have remarkable difference in their elasticities. Moreover, phantoms with viscoelasticity covering full range of bio-tissues could also be good references for the research with the interest of tissue characterization. Therefore, specific study on phantom fabrication and characterization could be helpful, and it is recommended as a future research topic.

\section{5) More In-vivo Experiments and Clinical Trials}

At last, in-vivo experiments in more subjects and at different body areas are recommended to carried out in future. Clinical trials could also be considered in even further stages. 


\section{Appendix A. Mode Conversion from Shear Wave to}

\section{Longitudinal Wave}

According to a wave mode conversion theory, an induced SW would not be totally reflected as SW during a reflection and partial energy of the induced SW would convert into a LW [119], [120]. Relevant equations were shown as follows.

$$
\begin{aligned}
& \Gamma_{S S}=-\frac{\sin 2 \theta_{S} \sin 2 \theta_{L}-\left(v_{L} / v_{S}\right)^{2} \cos ^{2} 2 \theta_{S}}{\sin 2 \theta_{S} \sin 2 \theta_{L}+\left(v_{L} / v_{S}\right)^{2} \cos ^{2} 2 \theta_{S}} \\
& \frac{\sin \theta_{L}}{\sin \theta_{S}}=\frac{v_{S}}{v_{L}},
\end{aligned}
$$

where $\Gamma_{S S}$ is the amplitude of a reflected SW from an incident SW. $v_{L}$ is the LW velocity of the specimen. Equation (A.2) follows the Snell's law which is described in Fig. A.1. $\theta_{S}$ is the incident angle of SW, and $\theta_{L}$ is the reflection angle of LW.

Using Eqs. (A.1) and (A.2), a numerical calculation was conducted to estimate the energy loss of the induced SW due to the mode conversion during the reflection. The value of $v_{S}$ was set from $1 \mathrm{~m} / \mathrm{s}$ to $10 \mathrm{~m} / \mathrm{s}$ with $0.1 \mathrm{~m} / \mathrm{s}$ step, and the $v_{L}$ was assumed at $1500 \mathrm{~m} / \mathrm{s}$. The reflection was assumed to be a total reflection. All the possible $\theta_{S}$ and $\theta_{L}$ were considered, then the energy conversion rate $\left|\Gamma_{S S}\right|^{2}$ from the incident SW to the reflected SW was calculated. The result was illustrated in Fig. A.2. Due to the great difference between $v_{S}$ and $v_{L}$, the critical incident angle (which is the maximum $\theta_{S}$ that

would lead the $\theta_{L}$ to $90^{\circ}$ ) was less than $0.4^{\circ}$, and at most, there would be only $1.8 \times 10^{-4} \%$ 
of the induced SW energy being converted into LW, which can be ignored. Therefore, it was assumed that in this thesis research, mode conversion was not involved.

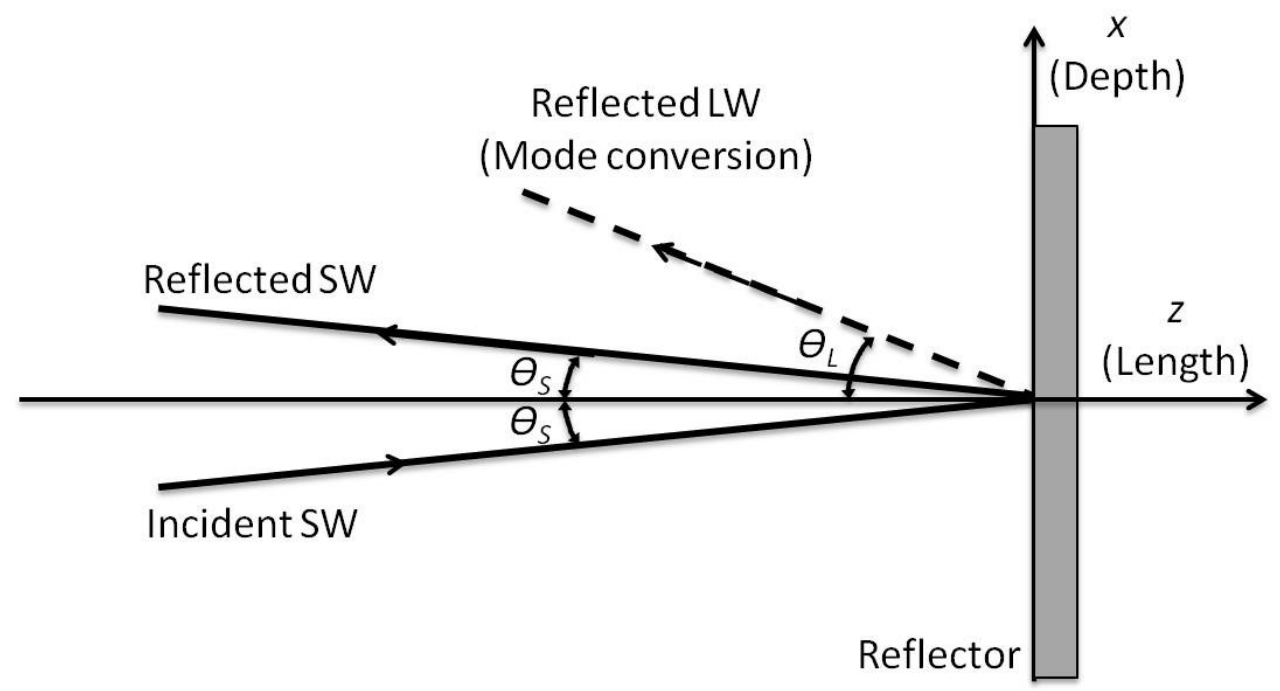

Figure A.1. Induced and reflected SWs with mode conversion.

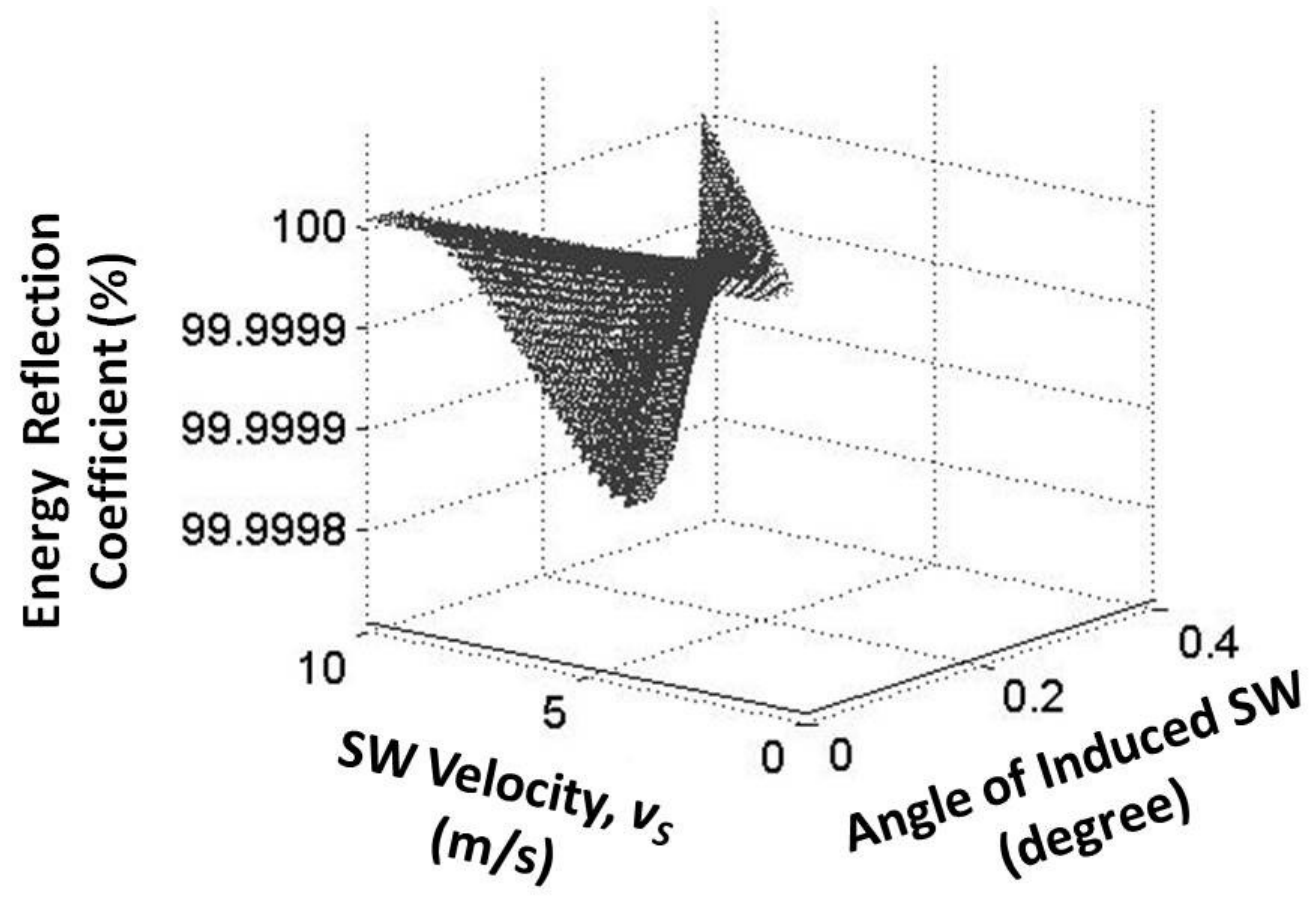

Figure A.2. Energy reflection coefficient of SW. 


\section{Appendix B. Measurement of Tissue Displacement}

\section{Using Ultrasound RF Signal}

Ultrasound was generated from the probe then reflected at the scatterers and acquired by the probe. Assume the transmitted ultrasound signal $S_{T}$ with a center frequency of $\omega_{0}$ was described as:

$$
S_{T}(t)=\cos \left(\omega_{0} t\right) .
$$

According to the principle of Doppler Effect, when an incident wave reached a moving target, the frequency of its reflection wave would experience a Doppler shift. Therefore, the received ultrasound $\mathrm{rf}$ signal $S_{r f}$, can be written as [121]:

$$
S_{r f}(t)=A_{r f} \cos \left[\int_{-\infty}^{t} \omega_{i}(t) d t\right],
$$

where $A_{r f}$ is the amplitude of the received rf signal, $\omega_{i}(t)=\omega_{0}+\omega_{d}(t)$, is the instantaneous angular frequency that is composed of the ultrasound frequency $\omega_{0}$ and the Doppler shift frequency $\omega_{d}(t)$ due to the displacement of the target (ultrasound scatterer). The Doppler shift frequency was defined by:

$$
\begin{aligned}
\omega_{d}(t) & =\frac{2 \omega_{0}}{c_{0}} \cos \theta \cdot v(t), \\
v(t)= & \frac{\partial u_{x}}{\partial t} \\
= & \omega_{S} A_{S} \cos \left(\omega_{S} t+\varphi_{S}\right),
\end{aligned}
$$


where $c_{0}$ is the ultrasound speed, $\theta$ is the angle between the direction of ultrasound transmission and the direction of the scatterer displacement. It should be noted that $\theta=0$ in this thesis. $u_{x}$ is the scatterer displacement as defined by Eq. (2.3). $\varphi_{S}$ is the phase of the velocity $(v(t))$ of the scatterer displacement. Then substituting Eqs. (B.3) and (B.4) into Eq. (B.2), the received rf signal can be rewritten as a frequency modulation (FM) equation [122]:

$$
S_{r f}(t)=A_{r f} \cos \left[\omega_{0} t+\varphi_{0}+\beta \cdot u_{x}(t)\right]
$$

where $\varphi_{0}$ is the initial phase of the received $\mathrm{rf}$ signal at $t=0$ when there is no motions. $\beta$, as expressed in Eq. (B.6), is the index of the FM and directly convert the amplitude of scatterer displacement (with a unit of meter) into phase component (with a unit of radian).

$$
\beta=\frac{2 \omega_{0}}{c_{0}}
$$

Now let us consider a three-dimensional displacement, where the scatterer displacement occurred at a $(x, z)$ spatial plane with respect to the time $t$, so Eq. (B.5) can be rewritten as:

$$
\begin{aligned}
& S_{r f}(t, x, z)=A_{r f}(x, z) \cos \left[\omega_{0} \frac{2 x}{c_{0}}+\varphi_{r f}(t, x, z)\right], \\
& \varphi_{r f}(t, x, z)=\varphi_{0}(x, z)+\varphi_{V}(t, x, z), \\
& \varphi_{V}(t, x, z)=\beta \cdot u_{x}(t, x, z),
\end{aligned}
$$

where $A_{r f}(x, z)$ is the amplitude of the $\mathrm{rf}$ signal at location $(x, z) .2 x / c_{0}$ is the time consumption of the pulse-echo round trip for an ultrasound pulse that travels from the probe to the target scatterer and back to the probe. The phase component $\varphi_{r}(t, x, z)$ represents the position of the scatterer located at $(x, z)$ at time $t$, which was composed of 
the initial phase $\left(\varphi_{0}(x, z)\right)$ of $\mathrm{rf}$ signal located at $(x, z)$ and $t=0$ when there was no scatterer motions. $\varphi_{\nu}(t, x, z)$ is the change of the phase, which was caused by the scatterer displacement $u_{x}(t, x, z)$.

Technically, $\varphi_{r f}(t, x, z)$ can be retrieved from the received $\mathrm{rf}$ signal using an inphase/quadrature detection technique at a known ultrasound transmission frequency $\omega_{0}$ [91], as follows:

$$
\begin{aligned}
S_{r e f}(x) & =e^{-\omega_{0} \frac{2 x}{c_{0}}}, \\
I(t, x, z) & =\operatorname{LPF}\left\{S_{r f}(t, x, z) \cdot \operatorname{Re}\left[S_{r e f}(x)\right]\right\} \\
& =\frac{A_{r f}(x, z)}{2} \cdot \operatorname{LPF}\left\{\cos \left[\varphi_{r f}(t, x, z)\right]+\cos \left[2 \omega_{0} \frac{2 x}{c_{0}}+\varphi_{r f}(t, x, z)\right]\right\} \\
& =\frac{A_{r f}(x, z)}{2} \cos \left[\varphi_{r f}(t, x, z)\right], \\
Q(t, x, z) & =L P F\left\{S_{r f}(t, x, z) \cdot \operatorname{Im}\left[S_{r e f}(x)\right]\right\} \\
& =\frac{A_{r f}(x, z)}{2} \cdot L P F\left\{\sin \left[\varphi_{r f}(t, x, z)\right]-\sin \left[2 \omega_{0} \frac{2 x}{c_{0}}+\varphi_{r f}(t, x, z)\right]\right\} \\
& =\frac{A_{r f}(x, z)}{2} \sin \left[\varphi_{r f}(t, x, z)\right], \\
Y(t, x, z) & =\frac{A_{r f}(x, z)}{2} e^{\varphi_{r f}(t, x, z)}, \\
Y(t, x, z) & =I(t, x, z)+j \cdot Q(t, x, z) \\
& =\angle Y(t, x, z),
\end{aligned}
$$

where $S_{r e f}$ is a complex reference signal with the same angular frequency as that of $S_{r f}$. The reference signal was multiplied to the rf signal to create an in-phase, denoted by $I(t, x$, 
$z$ ), and a quadrature signal, denoted by $Q(t, x, z)$. Low pass filtering $(L P F)$ was then applied on the in-phase and quadrature components, respectively.

Once the $\varphi_{r f}(t, x, z)$ was retrieved, the initial phase $\varphi_{0}(x, z)$ was considered as a phase offset and it can be removed by subtracting the mean value of the obtained $\varphi_{r f}(t, x$, $z$ ) over time $t$. Then $u_{x}(t, x, z)$ would be calculated by removing the FM index $\beta$. If the displacement due to motion artifact was taken into account, it can be reduced by applying a temporal frequency filter on the $u_{x}(t, x, z)$ with respect to $t$, as long as the $f_{S}$ employed in the experiment was separable from the frequencies of undesired motions. The details of such process were described in Chapter 5. 


\section{Appendix C. Undersampling of Tissue Displacement in Temporal Domain}

The ultrasound system used in this thesis had a B-mode scanning rate (or frame rate, $f_{F R}$ ) of $30 \mathrm{~Hz}$. However, the $f_{S}$ could be from around $100 \mathrm{~Hz}$ to $1000 \mathrm{~Hz}$ in practical experiment. This resulted in undersampling of the scatterer displacement in the temporal domain. Nevertheless, the undersampling in temporal domain did not affect the measurement of SW displacement, as long as the actual $f_{S}$ assigned did not make $f_{\text {measured }}$ become 0 (see Eq. (5.2) in section 5.2). If $f_{\text {measured }}$ became 0 , there would be no phase differences among the different moments of an arbitrary scatterer, i.e. the $\varphi_{r f}(t, x, z)$ obtained from Eq. (B.14) became a constant.

Figure C.1 demonstrated an example of SW propagation along $z$-direction at a certain depth $x$ with respect to different time $t$. Assuming such SW propagated towards the positive $z$-direction in spatial domain, and the sampling in its temporal domain was undersampled, while that in its spatial domain was normal sampled.

At a certain $z$, the displacement was caused by the term $\omega_{s} t$ in Eq. (2.3) and presented in Fig. C.1(b). At a certain $t$, the displacement was caused by the phase variation $k_{S} z$ in Eq. (2.3) and presented in Fig. C.1(c). As long as the sampled SW waveform in $z$-direction, indicated by the dash-dot in Fig. C.1(c), was not aliased, the wavenumber $k_{S}$ can be estimated correctly, so that $v_{S}$ would be estimated accordingly. 


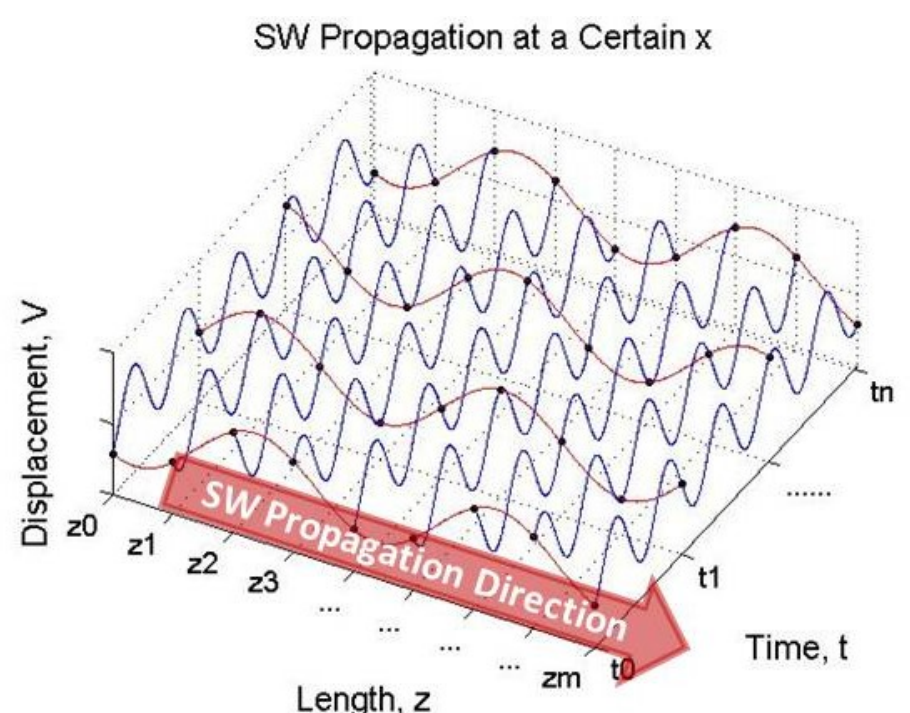

(a)

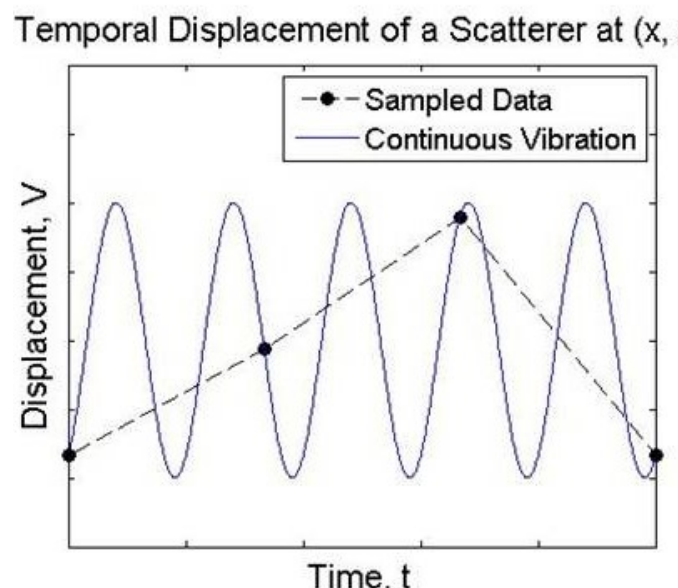

(b)

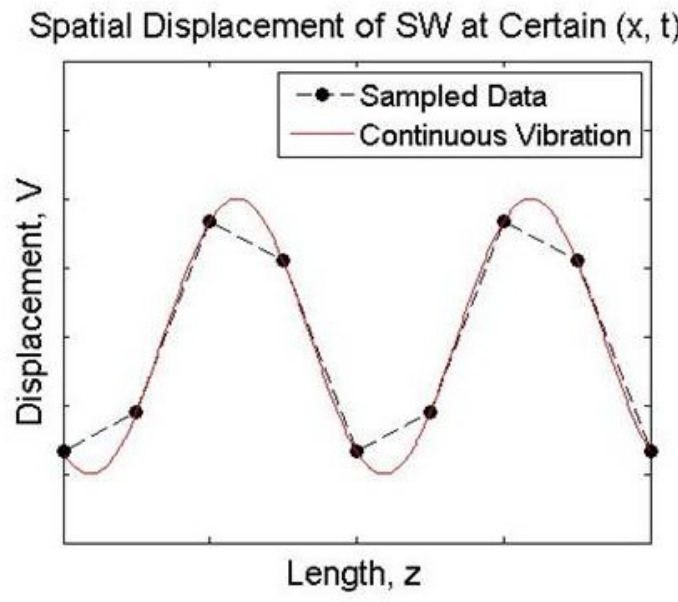

(c)

Figure C.1. A demonstration of SW displacement in (a) both temporal and spatial domain, (b) only temporal domain at a certain $(x, z)$, and (c) only spatial domain at a certain $(x, t)$. 


\section{Appendix D. Frequency-Domain Filtering}

The $\boldsymbol{B P F}_{\text {spatial }}$ used in Chapters $\mathbf{4}$ and $\mathbf{5}$ was a rectangular FIR with a filter order number of 42. With using such filter, $V, V_{B}$ at a certain frame of the ankle experiment demonstrated in Section 6.3.1, and their spatial frequency spectra at centerline $(x=0)$ were illustrated in Figs. D.1(a), (b), and (c), respectively. A strong frequency component was found at $0.55 \mathrm{~s} / \mathrm{m}$ in the spectrum of $V$ (indicated by dash-arrow in Fig. D.1(c)), which represented the dominant spatial frequency of the backward SW $V_{B}$. It can be seen that such frequency component was close to the lower spatial cut off frequency and probably located within the transition zone of the $\boldsymbol{B P} \boldsymbol{F}_{\text {spatial }}$. As a result, it was attenuated after the filtering process, and shifted to $0.77 \mathrm{~s} / \mathrm{m}$, as can be seen in the spectrum of $V_{B}$ (indicated by solid-arrow in Fig. D.1(c)). According to Eq. (4.9), a greater value of spatial frequency meant lower value of $v_{S}$, hence the $v_{S}$ estimated from the $V_{B}$ by using

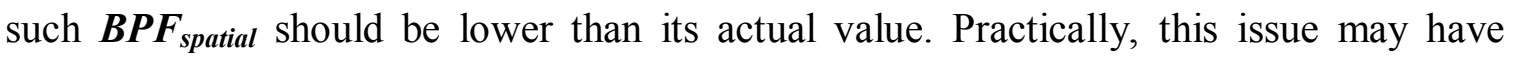
remarkable influence on the material that had a relatively greater $v_{S}$. In addition, the attenuation of the SW amplitude after the filtering process due to the transition zone also led to a weakening of the SNR, which would consequently reduce the accuracy of the $v_{S}$ estimation as presented in Fig. 5.9 of Section 5.5. 


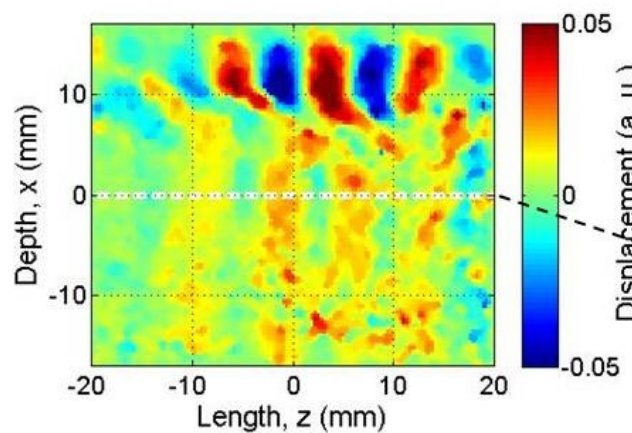

(a)

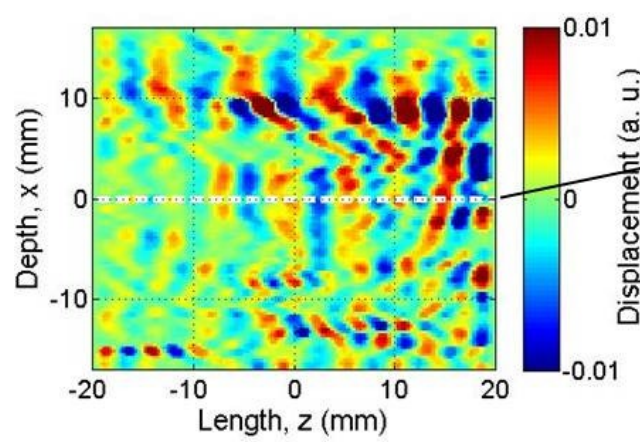

(b)

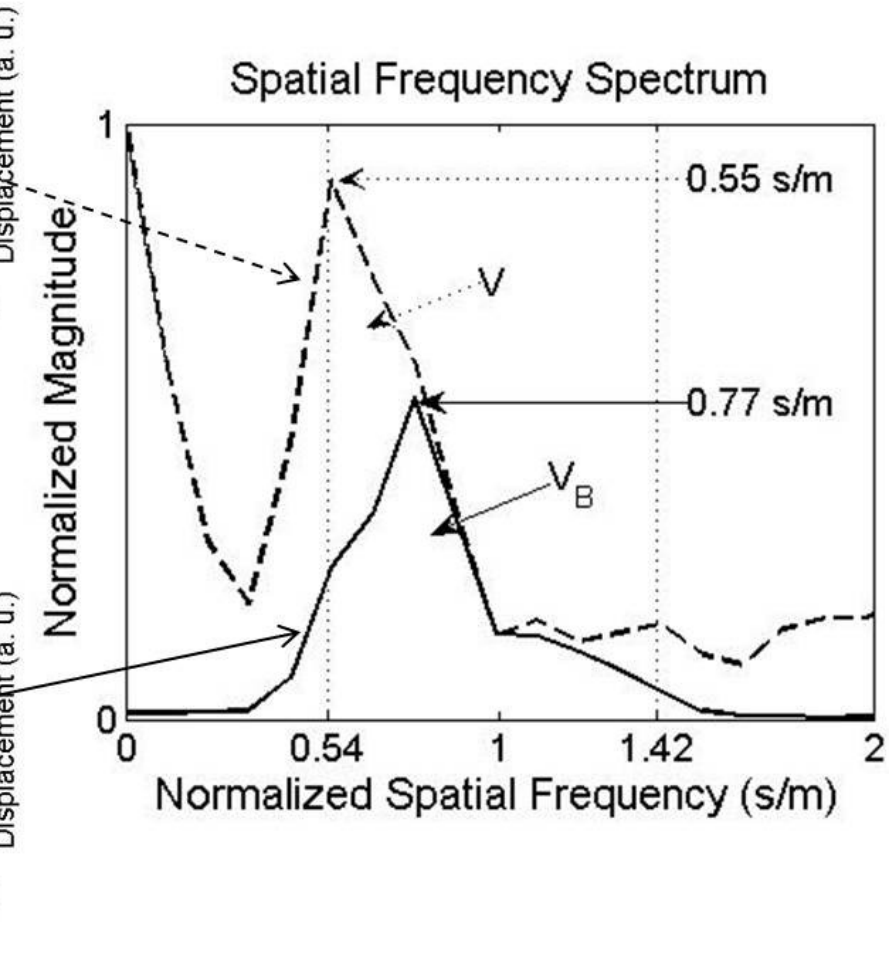

(c)

Figure D.1. The spatial SW displacement (a) before the spatial filtering, and (b) after the spatial filtering. The frequency spectrum at centerline $(x=0)$ of each displacement was averaged over 180 frames and plotted in (c).

An alternative $\boldsymbol{B P F}_{\text {spatial }}$ that applying on the frequency domain using the frequency domain filtering technique [113]-[116] may address such issue. The design of such $\boldsymbol{B P} \boldsymbol{F}_{\text {spatial }}$ followed the next steps:

\section{1) Fourier Transform:}

At every temporal frame, conducted Fourier transform of the obtained $V(x, z)$ in its spatial domain (with respect to $z$ ) at each depth $x$. Then calculated the frequency spectrum of the Fourier transform.

\section{2) Spatial Frequency Filtering:}


Applied a rectangular mask on the frequency spectrum, and made the magnitude zero for the spatial frequencies outside of the desired passband of the $\boldsymbol{B P} \boldsymbol{F}_{\text {spatial }}$. For the spatial frequencies within the passband, remained their original magnitudes.

\section{3) Inverse Fourier Transform:}

At last, conducted the inverse Fourier transform of the masked frequency spectrum at each depth $x$. Then the filtered backward SW displacement $V_{B}(x, z)$ would be obtained.

Theoretically this alternative $\boldsymbol{B P F}_{\text {spatial }}$ did not have transition zone in the spatial frequency domain. As long as the spatial frequency of SW was within the desired passband, it should be fully retained. However, it should be also noted that in practice, the sudden change of magnitudes at the cut-offs (assigning the magnitude to zero) in step 2 may lead to discontinuity in spatial frequency domain, and it may introduce errors such as ringing effects in the spatial domain. Improvement of the filtering process could be an interest topic in the future research.

Now the data of ankle experiment in Section 6.3.1 was reanalyzed by using the alternative $\boldsymbol{B P} \boldsymbol{F}_{\text {spatial }}$. In addition, considering the fact that the $v_{S}$ of Achilles tendon may be close to or greater than $10 \mathrm{~m} / \mathrm{s}$ [79], the passband of the alternative $\boldsymbol{B P F}_{\text {spatial }}$ were set to let a SW with $v_{S}$ between $2 \mathrm{~m} / \mathrm{s}$ and $15 \mathrm{~m} / \mathrm{s}$ pass,. Those presented in Fig. D.1 were reanalyzed and presented in Figs. D.2, accordingly. The dominant spatial frequency of the $V_{B}$ was now the same as that of $V$ within the passband. 


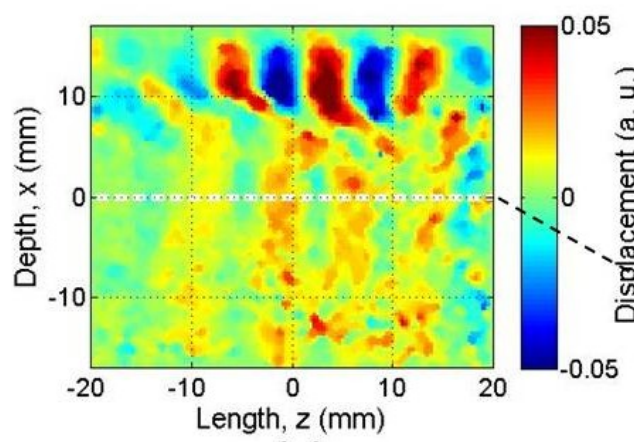

(a)

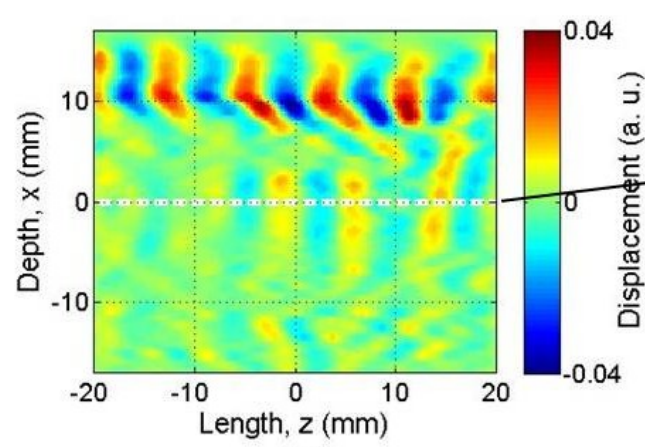

(b)

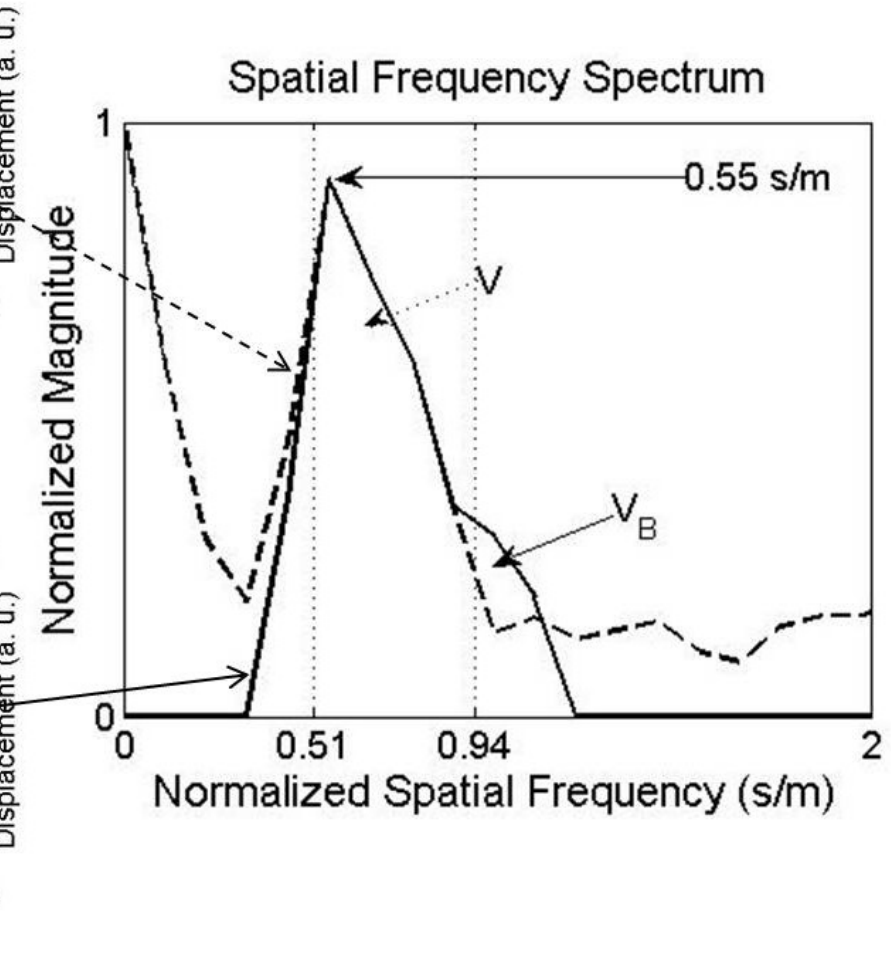

(c)

Figure D.2. The spatial SW displacement (a) before the spatial filtering, and (b) after the alternative spatial bandpass filtering. The frequency spectrum at centerline $(x=0)$ of each displacement was averaged over 180 frames and plotted in (c).

The $v_{S}$ was reestimated by using the alternative $\boldsymbol{B P} \boldsymbol{F}_{\text {spatial }}$ then averaged with 180 frames, and shown in Fig. D.3, the $v_{S}$ estimated beyond $2 \mathrm{~m} / \mathrm{s}$ to $15 \mathrm{~m} / \mathrm{s}$ was considered as calculation error so blackened and noted as N/A in the image. The mean value and STD of the $v_{S}$ of tendon tissue in the ROI were calculated $8.9 \mathrm{~m} / \mathrm{s}$ and $2.0 \mathrm{~m} / \mathrm{s}$, respectively. Those of muscle tissue were $5.1 \mathrm{~m} / \mathrm{s}$ and $1.8 \mathrm{~m} / \mathrm{s}$, respectively. Results of $v_{S}$ with two spatial filtering approaches were compared and listed in Table D.1. The "Difference" in the table was determined by: (Alternative $\boldsymbol{B P} \boldsymbol{F}_{\text {spatial }}-$ Original $\boldsymbol{B P} \boldsymbol{F}_{\text {spatial }}$ ) / Alternative $\boldsymbol{B P F}_{\text {spatial }} \times 100 \%$. 


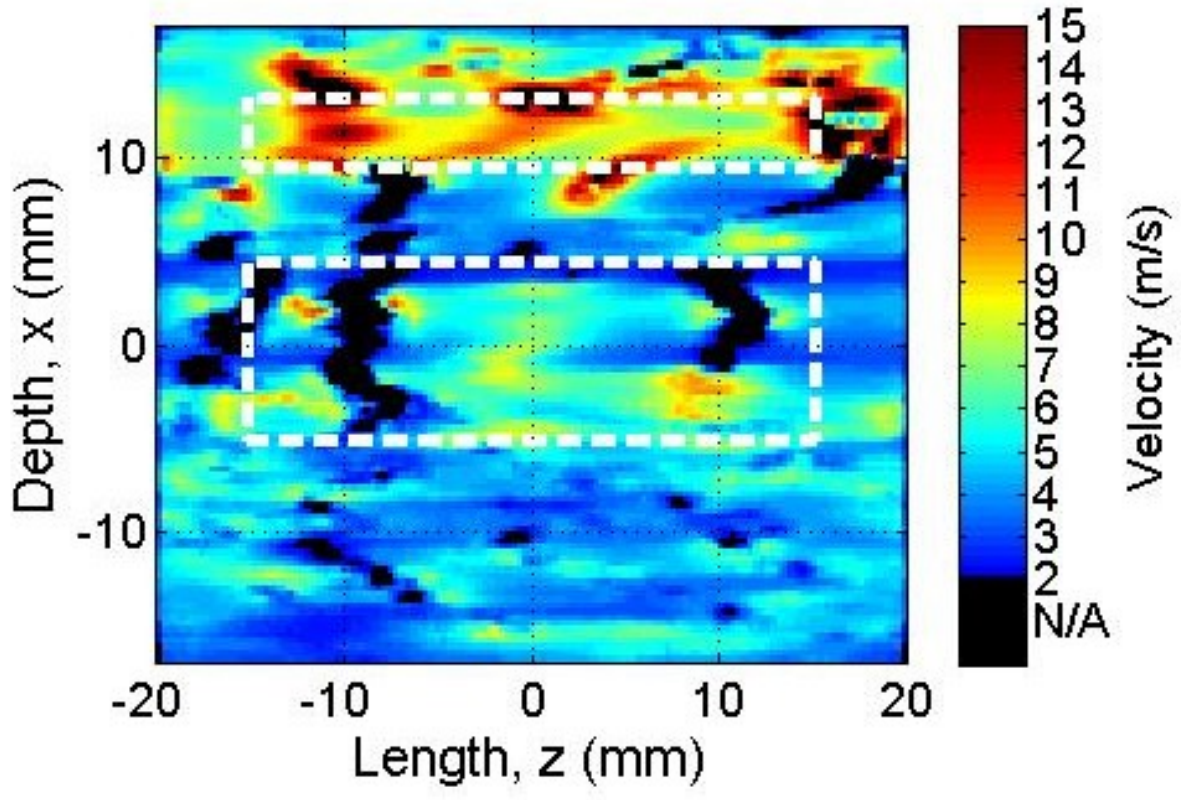

Figure D.3. SW velocity calculated with the alternative spatial filtering.

Table D.1. Shear velocities calculated using original $\boldsymbol{B P F}_{\text {spatial }}$ and alternative $\boldsymbol{B P F}_{\text {spatial }}$.

\begin{tabular}{|c|c|c|c|c|c|c|}
\hline & \multicolumn{2}{|c|}{ Original $\boldsymbol{B P F}_{\text {spatial }}$} & \multicolumn{2}{c|}{ Alternative $\boldsymbol{B P F}_{\text {spatial }}$} & \multicolumn{2}{c|}{ Difference } \\
\cline { 2 - 7 } & Tendon & Muscle & Tendon & Muscle & Tendon & Muscle \\
\hline $\begin{array}{c}\text { Mean value of } \\
\boldsymbol{v}_{\boldsymbol{S}}(\mathbf{m} / \mathbf{s})\end{array}$ & 4.6 & 2.5 & 8.9 & 5.1 & $+48 \%$ & $+51 \%$ \\
\hline $\begin{array}{c}\text { Standard } \\
\text { deviation (m/s) }\end{array}$ & 1.7 & 0.75 & 2.0 & 1.8 & $+15 \%$ & $+58 \%$ \\
\hline
\end{tabular}

The $v_{S}$ of both tendon and muscle tissues were calculated at greater values when using the alternative $\boldsymbol{B P} \boldsymbol{F}_{\text {spatial }}$. The $v_{S}$ of tendon tissue showed good agreement with the published results [33], [79]. As mentioned earlier, the alternative $\boldsymbol{B P F}_{\text {spatial }}$ caused sudden change of magnitudes at the cut-offs and resulted in discontinuity in spatial frequency domain. Therefore, variation of the $v_{S}$ image can be noticed in Fig. D.3, so that the calculation errors (blacken areas) appeared at $z=0 \mathrm{~mm}, \pm 10 \mathrm{~mm}$, and $\pm 15 \mathrm{~mm}$ could be 
due to this issue. In Fig. D.4, the $v_{S}$ image was superimposed on the B-mode image. It can be seen that the $v_{S}$ difference between tendon and other tissues followed their anatomical structures.

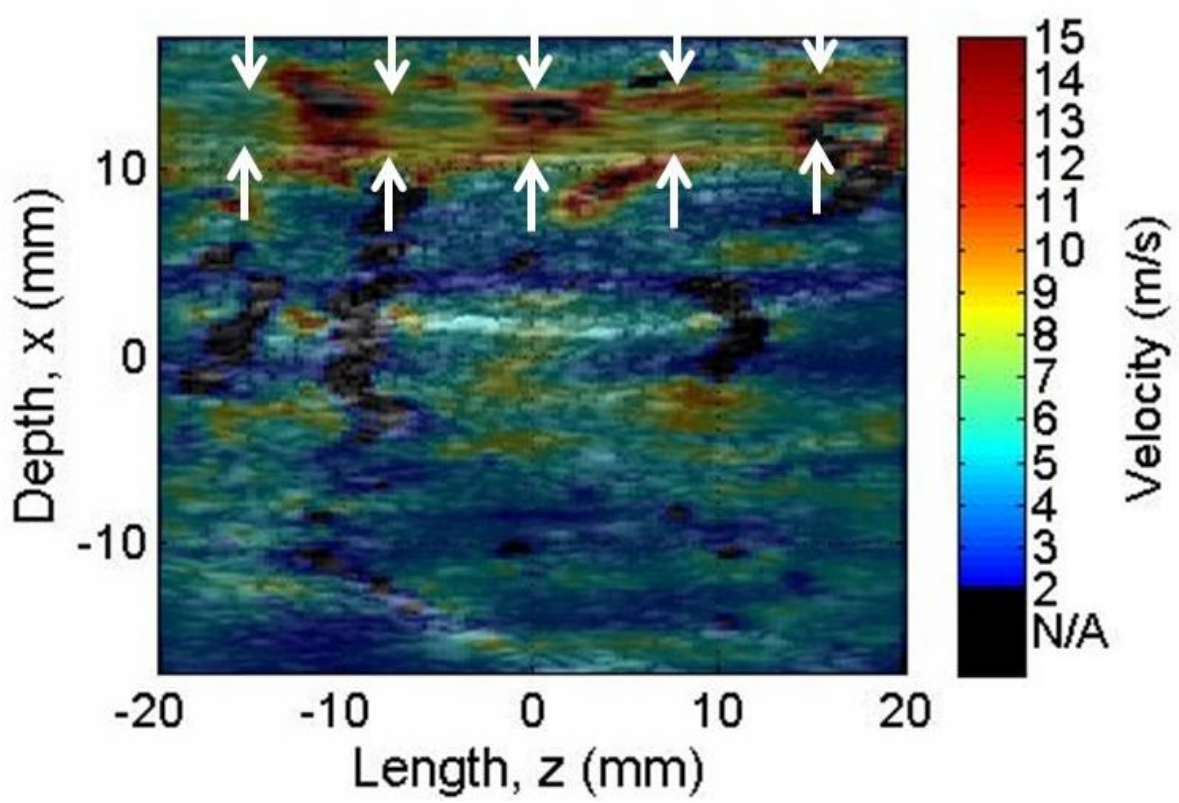

Figure D.4. A superposition of B-mode image and SW velocity image. Arrows indicated the Achilles tendon.

The results of $v_{S}$ in tendon and muscle areas averaged with 10 consecutive frames as those demonstrated in Fig. 6.5 were recalculated with using the alternative $\boldsymbol{B P F}_{\text {spatial }}$, and shown in Fig. D.5. Both $v_{S}$ results were apart (as shown in Table D.1) with a certain amount value and they were consistent throughout the SW measurement. The $v_{S}$ variation (twice of the STD) in Fig. D.5(a) was $0.15 \mathrm{~m} / \mathrm{s}$ and that in Fig. D.5(b) was $0.29 \mathrm{~m} / \mathrm{s}$. 


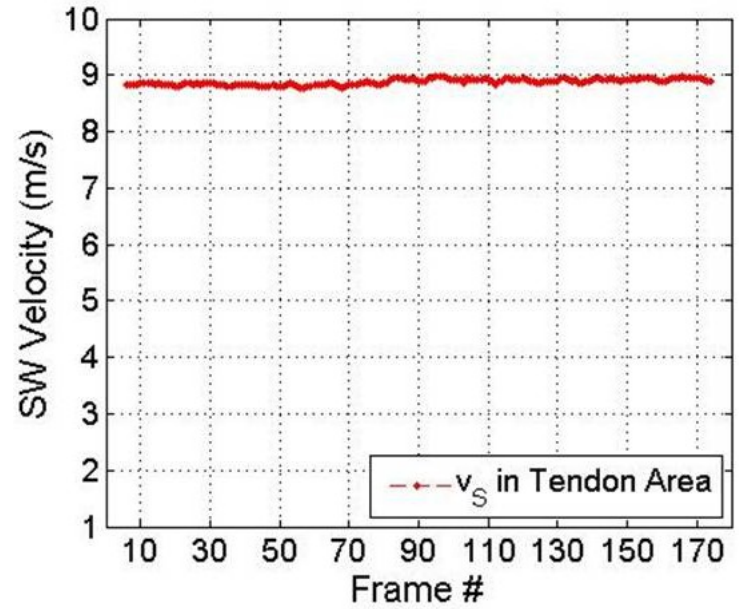

(a)

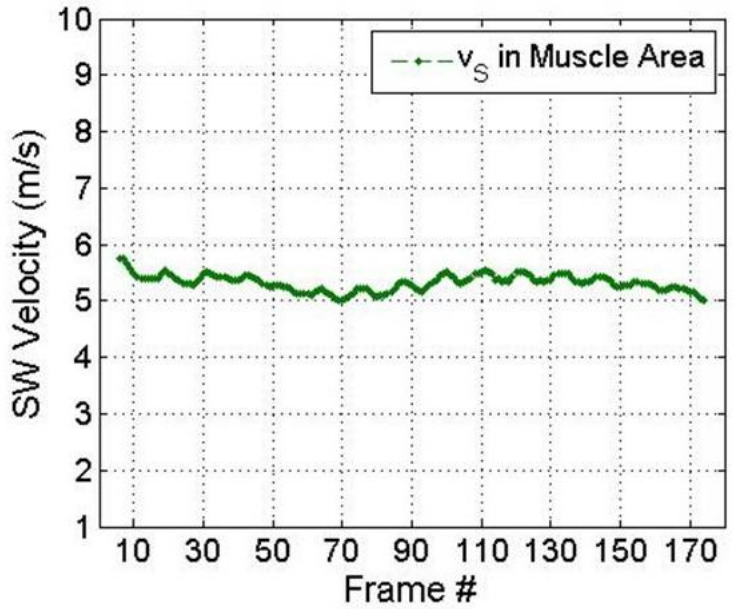

(b)

Figure D.5. The change of the mean value of the SW velocity with respect to the temporal frames within the ROIs as indicated by the dash-box in Fig. D.3. The result in the ROI of tendon was plotted in (a) and that of muscle was plotted in (b). Both results were calculated by using the alternative $\boldsymbol{B P F}_{\text {spatial }}$. 


\section{List of References}

[1] J. Ophir, I. Cespedes, H. Ponnekanti, Y. Yazdi, and X. Li, "Elastography: A Quantitative Method for Imaging the Elasticicty of Biological Tissues.," Ultrasonic Imaging, vol. 13, no. 2. pp. 111-134, 1991.

[2] N. Salameh, F. Peeters, R. Sinkus, J. Abarca-Quinones, L. Annet, L. C. Ter Beek, I. Leclercq, and B. E. Van Beers, "Hepatic viscoelastic parameters measured with MR elastography: Correlations with quantitative analysis of liver fibrosis in the rat," J. Magn. Reson. Imaging, vol. 26, no. 4, pp. 956-962, 2007.

[3] L. Huwart, F. Peeters, R. Sinkus, L. Annet, N. Salameh, L. C. ter Beek, Y. Horsmans, and B. E. Van Beers, "Liver fibrosis: Non-invasive assessment with MR elastography," NMR Biomed., vol. 19, no. 2, pp. 173-179, 2006.

[4] L. Gao, K. J. Parker, R. M. Lerner, and S. F. Levinson, "Imaging of the elastic properties of tissue--a review.," Ultrasound Med. Biol., vol. 22, no. 8, pp. 959-977, 1996.

[5] H. Eskandari, S. E. Salcudean, and R. Rohling, "Viscoelastic parameter estimation based on spectral analysis.," IEEE Trans. Ultrason. Ferroelectr. Freq. Control, vol. 55, no. 7, pp. 1611-1625, 2008.

[6] J. Ophir, S. K. Alam, B. Garra, F. Kallel, E. Konofagou, T. Krouskop, and T. Varghese, "Elastography: ultrasonic estimation and imaging of the elastic properties of tissues.," Proc. Inst. Mech. Eng. H., vol. 213, pp. 203-233, 1999.

[7] Y. Yamakoshi, J. Sato, and T. Sato, "Ultrasonic Imaging of Internal Vibration of Soft Tissue under Forced Vibration," IEEE Trans. Ultrason. Ferroelectr. Freq. Control, vol. 37, no. 2, pp. 45-53, 1990.

[8] R. Muthupillai, D. J. Lomas, P. J. Rossman, J. F. Greenleaf, a Manduca, and R. L. 
Ehman, "Magnetic resonance elastography by direct visualization of propagating acoustic strain waves.," Science, vol. 269, no. 5232, pp. 1854-1857, 1995.

[9] A. Manduca, R. Muthupillai, P. J. J. Rossman, J. F. F. Greenleaf, and R. L. L. Ehman, "Image analysis for magnetic resonance elastography," Proc. 18th Annu. Int. Conf. IEEE Eng. Med. Biol. Soc., vol. 2, pp. 756-757, 1996.

[10] A. Sarvazyan, O. Rudenko, and S. Swanson, "Shear wave elasticity imaging: a new ultrasonic technology of medical diagnostics," Ultrasound Med. Biol., vol. 24, no. 9, pp. 1419-1435, 1998.

[11] M. Fatemi and J. F. Greenleaf, "Ultrasound-stimulated vibro-acoustic spectrography.," Science, vol. 280, no. 5360, pp. 82-85, 1998.

[12] M. a Dresner, G. H. Rose, P. J. Rossman, R. Muthupillai, a Manduca, and R. L. Ehman, "Magnetic resonance elastography of skeletal muscle.," J. Magn. Reson. Imaging, vol. 13, pp. 269-276, 2001.

[13] S. Chen, M. Fatemi, and J. F. Greenleaf, "Shear property characterization of viscoelastic media using vibrations induced by ultrasound radiation force," 2002 IEEE Ultrason. Symp. 2002. Proceedings., vol. 2, no. c, pp. 1871-1875, 2002.

[14] J. F. Greenleaf, M. Fatemi, and M. Insana, "Selected methods for imaging elastic properties of biological tissues.," Annu. Rev. Biomed. Eng., vol. 5, pp. 57-78, 2003.

[15] J. Bercoff, M. Muller, M. Tanter, and M. Fink, "Study of viscous and elastic properties of soft tissues using supersonic shear imaging," IEEE Symp. Ultrason. 2003, vol. 1, no. c, pp. 925-928, 2003.

[16] J. Bercoff, M. Tanter, and M. Fink, "Supersonic Shear Imaging: A New Technique," IEEE Trans. Ultrason. Ferroelectr. Freq. Control, vol. 51, no. 4, pp. 396-409, 2004.

[17] S. Chen, M. Fatemi, and J. F. Greenleaf, "Quantifying elasticity and viscosity from measurement of shear wave speed dispersion.," J. Acoust. Soc. Am., vol. 115, no. 6, pp. 2781-2785, 2004.

[18] R. Sinkus, M. Tanter, S. Catheline, J. Lorenzen, C. Kuhl, E. Sondermann, and M. 
Fink, "Imaging anisotropic and viscous properties of breast tissue by magnetic resonance-elastography," Magn. Reson. Med., vol. 53, no. 2, pp. 372-387, 2005.

[19] Z. Wu, K. Hoyt, D. J. Rubens, and K. J. Parker, "Sonoelastographic imaging of interference patterns for estimation of shear velocity distribution in biomaterials.," J. Acoust. Soc. Am., vol. 120, no. 1, pp. 535-545, 2006.

[20] S. Chen, M. W. Urban, C. Pislaru, R. Kinnick, Y. Zheng, A. Yao, and J. F. Greenleaf, "Shearwave dispersion ultrasound vibrometry (SDUV) for measuring tissue elasticity and viscosity," IEEE Trans. Ultrason. Ferroelectr. Freq. Control, vol. 56, no. 1, pp. 55-62, 2009.

[21] T. Deffieux, G. Montaldo, M. Tanter, and M. Fink, "Shear wave spectroscopy for in vivo quantification of human soft tissues visco-elasticity," IEEE Trans. Med. Imaging, vol. 28, no. 3, pp. 313-322, 2009.

[22] C. Hazard, Z. Hah, D. Rubens, and K. Parker, "Integration of Crawling Waves in an Ultrasound Imaging System. Part 1: System and Design Considerations," Ultrasound Med. Biol., vol. 38, no. 2, pp. 296-311, 2012.

[23] Z. Hah, C. Hazard, B. Mills, C. Barry, D. Rubens, and K. Parker, "Integration of crawling waves in an ultrasound imaging system. Part 2: Signal processing and applications," Ultrasound Med. Biol., vol. 38, no. 2, pp. 312-323, 2012.

[24] P. Song, M. W. Urban, A. Manduca, H. Zhao, J. F. Greenleaf, and S. Chen, "Comb-push Ultrasound Shear Elastography (CUSE): A novel and fast technique for shear elasticity imaging," IEEE Int. Ultrason. Symp. IUS, vol. 31, no. 9, pp. 1842-1845, 2012.

[25] Z. Qu and Y. Ono, "A method to reduce the influence of reflected waves on shear velocity measurements using B-mode scanning time delay," Jpn. J. Appl. Phys., vol. 54, no. 7S1, p. 07HF01, 2015.

[26] L. Castéra, J. Vergniol, J. Foucher, B. Le Bail, E. Chanteloup, M. Haaser, M. Darriet, P. Couzigou, and V. De Lédinghen, "Prospective comparison of transient elastography, Fibrotest, APRI, and liver biopsy for the assessment of fibrosis in chronic hepatitis C," Gastroenterology, vol. 128, no. 2, pp. 343-350, 2005. 
[27] L. Huwart, C. Sempoux, N. Salameh, J. Jamart, L. Annet, R. Sinkus, F. Peeters, L. C. ter Beek, Y. Horsmans, and B. E. Van Beers, "Liver fibrosis: noninvasive assessment with MR elastography versus aspartate aminotransferase-to-platelet ratio index.," Radiology, vol. 245, no. 2, pp. 458-466, 2007.

[28] M. Zhang, P. Nigwekar, B. Castaneda, K. Hoyt, J. V Joseph, A. di Sant'Agnese, E. M. Messing, J. G. Strang, D. J. Rubens, and K. J. Parker, "Quantitative characterization of viscoelastic properties of human prostate correlated with histology.," Ultrasound Med. Biol., vol. 34, no. 7, pp. 1033-42, 2008.

[29] A. Athanasiou, A. Tardivon, M. Tanter, B. Sigal-Zafrani, J. Bercoff, T. Deffieux, J.-L. Gennisson, M. Fink, and S. Neuenschwander, "Breast lesions: quantitative elastography with supersonic shear imaging--preliminary results.," Radiology, vol. 256, no. 1, pp. 297-303, 2010.

[30] A. Evans, P. Whelehan, K. Thomson, D. McLean, K. Brauer, C. Purdie, L. Jordan, L. Baker, and A. Thompson, "Quantitative shear wave ultrasound elastography: initial experience in solid breast masses.," Breast Cancer Res., vol. 12, no. 6, p. R104, 2010.

[31] I. Nenadic, M. Mehrmohammadi, M. W. Urban, A. Alizad, J. F. Greenleaf, and M. Fatemi, "In vivo patient measurements of bladder elasticity using Ultrasound Bladder Vibrometry (UBV)," Proc. Annu. Int. Conf. IEEE Eng. Med. Biol. Soc. EMBS, pp. 113-116, 2013.

[32] I. Z. Nenadic, B. Qiang, M. W. Urban, L. H. de Araujo Vasconcelo, A. Nabavizadeh, A. Alizad, J. F. Greenleaf, and M. Fatemi, "Ultrasound bladder vibrometry method for measuring viscoelasticity of the bladder wall.," Phys. Med. Biol., vol. 58, no. 8, pp. 2675-95, 2013.

[33] D. H. Cortes, S. M. Suydam, K. G. Silbernagel, T. S. Buchanan, and D. M. Elliott, "Continuous Shear Wave Elastography: A New Method to Measure Viscoelastic Properties of Tendons in Vivo," Ultrasound Med. Biol., vol. 41, no. 6, pp. 15181529, 2015.

[34] Z. Wu, L. S. Taylor, D. J. Rubens, and K. J. Parker, "Sonoelastographic imaging 
of interference patterns for estimation of the shear velocity of homogeneous biomaterials," Phys. Med. Biol., vol. 49, no. 6, p. 911, 2004.

[35] A. Manduca, D. S. Lake, S. a. Kruse, and R. L. Ehman, "Spatio-temporal directional filtering for improved inversion of MR elastography images," Med. Image Anal., vol. 7, no. 4, pp. 465-473, 2003.

[36] T. Deffieux, J. L. Gennisson, J. Bercoff, and M. Tanter, "On the effects of reflected waves in transient shear wave elastography," IEEE Trans. Ultrason. Ferroelectr. Freq. Control, vol. 58, no. 10, pp. 2032-2035, 2011.

[37] A. J. Engel and G. R. Bashford, "A new method for shear wave speed estimation in shear wave elastography," Ultrason. Ferroelectr. Freq. Control. IEEE Trans., vol. 62, no. 12, pp. 2106-2114, 2015.

[38] J. Silver, Y. Ono, and A. Adler, "An ultrasonic technique for imaging of tissue motion due to muscle contraction," Proc. - IEEE Ultrason. Symp., pp. 2441-2444, 2009.

[39] R. Chandrasekhar, J. Ophir, T. Krouskop, and K. Ophir, "Elastographic image quality vs. tissue motion in vivo," Ultrasound Med. Biol., vol. 32, no. 6, pp. 847855, 2006.

[40] J. W. Goodman, Introduction to Fourier Optics, 2nd ed. New York: McGraw-Hill, 1996.

[41] A. P. Sarvazyan, A. R. Skovoroda, S. Y. Emelianov, J. B. Fowlkes, J. G. Pipe, R. S. Adler, R. B. Buxton, and P. L. Carson, "Biophysical Bases of Elasticity Imaging," in Acoustical Imaging, J. P. Jones, Ed. Boston, MA: Springer US, 1995, pp. 223240.

[42] M. A. Meyers and K. K. Chawla, Mechanical Behavior of Materials, 2nd ed. New York: The Cambridge University Press, 2009.

[43] J. Ophir, I. Cespedes, B. Garra, H. Ponnekanti, and Y. Huang, "Elastography Ultrasonic imaging of tissue strain and elastic modulus in vivo.pdf," vol. 8266, no. 95, 1996. 
[44] J. D. N. Cheeke, Fundamentals and Applications. Boca Raton: CRC Press LLC, 2002.

[45] K. J. Parker, M. M. Doyley, and D. J. Rubens, "Imaging the elastic properties of tissue: the 20 year perspective," Phys. Med. Biol., vol. 57, pp. 5359-5360, 2012.

[46] H. Schiessel, R. Metzler, A. Blumen, and T. F. Nonnenmacher, "Generalized viscoelastic models: their fractional equations with solutions," J. Phys. A. Math. Gen., vol. 28, no. 23, pp. 6567-6584, 1995.

[47] R. Darby, Chemical Engineering Fluid Mechanics, 2nd ed. Basel: Marcel Dekker, 2004.

[48] E. Madsen, H. Sathoff, and J. Zagzebski, "Ultrasonic shear wave properties of soft tissues and tissuelike materials," J. Acoust. Soc. Am., vol. 74, no. 5, pp. 1346-1355, 1983.

[49] S. G. Chen, M. Fatemi, and J. F. Greenleaf, "Remote measurement of material properties from radiation force induced vibration of an embedded sphere," $J$. Acoust. Soc. Am., vol. 112, no. 3, pp. 884-889, 2002.

[50] S. A. McAleavey, M. Menon, and J. Orszulak, "Shear-modulus estimation by application of spatially-modulated impulsive acoustic radiation force.," Ultrason. Imaging, vol. 29, no. 2, pp. 87-104, 2007.

[51] H. L. Oestreicher, "Field and impedance of an oscillating sphere in a viscoelastic medium with an application to biophysics," J. Acoust. Soc. Am., vol. 23, no. 6, pp. 1033-1042, 1951.

[52] T. E. Oliphant, "Direct Methods for Dynamic Elastography Reconstruction: Optimal Inversion of the Interior Helmholtz Problem," Mayo Graduate School, 2001.

[53] S. K. Venkatesh, M. Yin, and R. L. Ehman, "Magnetic resonance elastography of liver: technique, analysis, and clinical applications.," J. Magn. Reson. Imaging, vol. 37, no. 3, pp. 544-55, 2013.

[54] A. Manduca, T. E. Oliphant, M. A. Dresner, J. L. Mahowald, S. A. Kruse, E. 
Amromin, J. P. Felmlee, J. F. Greenleaf, and R. L. Ehman, "Magnetic resonance elastography: Non-invasive mapping of tissue elasticity," Med. Image Anal., vol. 5, no. 4, pp. 237-254, 2001.

[55] L. Huwart, C. Sempoux, E. Vicaut, N. Salameh, L. Annet, E. Danse, F. Peeters, L. C. ter Beek, J. Rahier, R. Sinkus, Y. Horsmans, and B. E. Van Beers, "Magnetic Resonance Elastography for the Noninvasive Staging of Liver Fibrosis," Gastroenterology, vol. 135, no. 1, pp. 32-40, 2008.

[56] A. Samani, J. Zubovits, and D. Plewes, "Elastic moduli of normal and pathological human breast tissues: an inversion-technique-based investigation of 169 samples," Phys. Med. Biol, vol. 52, pp. 1565-1576, 2007.

[57] R. Sinkus, J. Lorenzen, D. Schrader, M. Lorenzen, M. Dargatz, and D. Holz, "High-resolution tensor MR elastography for breast tumour detection.," Phys. Med. Biol., vol. 45, no. 6, pp. 1649-1664, 2000.

[58] N. B. Delongchamps, M. Peyromaure, A. Schull, F. Beuvon, N. Bouazza, T. Flam, M. Zerbib, N. Muradyan, P. Legman, and F. Cornud, "Prebiopsy magnetic resonance imaging and prostate cancer detection: comparison of random and targeted biopsies.," J. Urol., vol. 189, no. 2, pp. 493-9, 2013.

[59] J. Chappelow, B. N. Bloch, N. Rofsky, E. Genega, R. Lenkinski, W. DeWolf, and A. Madabhushi, "Elastic registration of multimodal prostate MRI and histology via multiattribute combined mutual information.," Med. Phys., vol. 38, no. 4, pp. 2005-2018, 2011.

[60] S. A. Kruse, G. H. Rose, K. J. Glaser, A. Manduca, J. P. Felmlee, C. R. Jack, and R. L. Ehman, "Magnetic resonance elastography of the brain," Neuroimage, vol. 39, no. 1, pp. 231-237, 2008.

[61] J. Braun, J. Guo, R. Lützkendorf, J. Stadler, S. Papazoglou, S. Hirsch, I. Sack, and J. Bernarding, "High-resolution mechanical imaging of the human brain by threedimensional multifrequency magnetic resonance elastography at 7T," Neuroimage, vol. 90, pp. 308-314, 2014.

[62] C. Vorländer, J. Wolff, S. Saalabian, R. H. Lienenlüke, and R. A. Wahl, "Real- 
time ultrasound elastography-a noninvasive diagnostic procedure for evaluating dominant thyroid nodules," Langenbeck's Arch. Surg., vol. 395, no. 7, pp. 865$871,2010$.

[63] F. Sebag, J. Vaillant-Lombard, J. Berbis, V. Griset, J. F. Henry, P. Petit, and C. Oliver, "Shear wave elastography: a new ultrasound imaging mode for the differential diagnosis of benign and malignant thyroid nodules.," J. Clin. Endocrinol. Metab., vol. 95, no. 12, pp. 5281-8, 2010.

[64] D. Cosgrove, F. Piscaglia, J. Bamber, J. Bojunga, J.-M. Correas, O. H. Gilja, A. S. Klauser, and D. O. Cosgrove, "EFSUMB Guidelines and Recommendations on the Clinical Use of Ultrasound Elastography. Part 2: Clinical Applications," Ultraschall Med, vol. 34, pp. 238-253, 2013.

[65] E. E. Drakonaki, G. M. Allen, and D. J. Wilson, "Ultrasound elastography for musculoskeletal applications," Br. J. Radiol., vol. 85, no. 1019, pp. 1435-1445, 2012.

[66] B. C. W. Kot, Z. J. Zhang, A. W. C. Lee, V. Y. F. Leung, and S. N. Fu, "Elastic Modulus of Muscle and Tendon with Shear Wave Ultrasound Elastography: Variations with Different Technical Settings," PLoS One, vol. 7, no. 8, pp. 2-7, 2012.

[67] T. Muraki, H. Ishikawa, S. Morise, N. Yamamoto, H. Sano, E. Itoi, and S. ichi Izumi, "Ultrasound elastography-based assessment of the elasticity of the supraspinatus muscle and tendon during muscle contraction," J. Shoulder Elb. Surg., vol. 24, no. 1, pp. 120-126, 2015.

[68] E. Turgay, S. Salcudean, and R. Rohling, "Identifying the mechanical properties of tissue by ultrasound strain imaging," Ultrasound Med. Biol., vol. 32, no. 2, pp. 221-235, 2006.

[69] M. Sridhar, J. Liu, and M. F. Insana, "Viscoelasticity imaging using ultrasound: parameters and error analysis.," Phys. Med. Biol., vol. 52, no. 9, pp. 2425-2443, 2007.

[70] R. M. Lerner, K. J. Parker, J. Holen, R. Gramiak, and R. C. Waag, "Sono- 
Elasticity: Medical Elasticity Images Derived from Ultrasound Signals in Mechanically Vibrated Targets," in Acoustical Imaging: Proceedings of the Sixteenth International Symposium, June 10--12, 1987, L. W. Kessler, Ed. Boston, MA: Springer US, 1988, pp. 317-327.

[71] S. Catheline, F. Wu, and M. Fink, "A solution to diffraction biases in sonoelasticity: the acoustic impulse technique.," J. Acoust. Soc. Am., vol. 105, no. 5, pp. 2941-2950, 1999.

[72] K. R. Nightingale, M. L. Palmeri, R. W. Nightingale, and G. E. Trahey, "On the feasibility of remote palpation using acoustic radiation force.," J. Acoust. Soc. Am., vol. 110, no. 1, pp. 625-34, 2001.

[73] P. Song, M. C. Macdonald, R. H. Behler, J. D. Lanning, M. H. Wang, M. W. Urban, A. Manduca, H. Zhao, M. R. Callstrom, A. Alizad, J. F. Greenleaf, and S. Chen, "Shear wave elastography on the GE LOGIQ E9 with Comb-push Ultrasound Shear Elastography (CUSE) and time aligned sequential tracking (TAST)," in IEEE International Ultrasonics Symposium, IUS, 2014, vol. 1, pp. 1101-1104.

[74] P. Song, M. Macdonald, R. Behler, J. Lanning, M. Wang, M. Urban, A. Manduca, H. Zhao, M. Callstrom, A. Alizad, J. Greenleaf, and S. Chen, "Two-dimensional shear-wave elastography on conventional ultrasound scanners with time-aligned sequential tracking (TAST) and comb-push ultrasound shear elastography (CUSE)," IEEE Trans. Ultrason. Ferroelectr. Freq. Control, vol. 62, no. 2, pp. 290-302, 2015.

[75] U. Zaleska-Dorobisz, K. Kaczorowski, A. Pawlu??, A. Puchalska, and M. Inglot, "Ultrasound elastography - review of techniques and its clinical applications.," $A d v$. Clin. Exp. Med., vol. 23, no. 4, pp. 645-655, 2014.

[76] T. Deffieux, J.-L. Gennisson, L. Bousquet, M. Corouge, S. Cosconea, D. Amroun, S. Tripon, B. Terris, V. Mallet, P. Sogni, M. Tanter, and S. Pol, "Investigating liver stiffness and viscosity for fibrosis, steatosis and activity staging using shear wave elastography.," J. Hepatol., vol. 62, no. 2, pp. 317-24, 2015. 
[77] J. Ophir, S. K. Alam, B. S. Garra, F. Kallel, E. E. Konofagou, T. Krouskop, C. R. B. Merritt, R. Righetti, R. Souchon, S. Srinivasan, and T. Varghese, "Elastography: Imaging the elastic properties of soft tissues with ultrasound," J. Med. Ultrason., vol. 29 , no. 4, pp. 155-171, 2002.

[78] J. Zhou, W. Zhan, C. Chang, J. Zhang, Z. Yang, Y. Dong, C. Zhou, and Y. Song, "Role of Acoustic Shear Wave Velocity Measurement in Characterization of Breast Lesions," J. Ultrasound Med., vol. 32, no. 2, pp. 285-294, 2013.

[79] J. Brum, J. L. Gennisson, and M. Tanter, "In vivo evaluation of the elastic anisotropy of the human Achilles tendon using shear wave dispersion analysis," Phys. Med. Biol., vol. 59, pp. 505-523, 2014.

[80] S. Laurent, J. Cockcroft, L. Van Bortel, P. Boutouyrie, C. Giannattasio, D. Hayoz, B. Pannier, C. Vlachopoulos, I. Wilkinson, and H. Struijker-Boudier, "Expert consensus document on arterial stiffness: Methodological issues and clinical applications," Eur. Heart J., vol. 27, no. 21, pp. 2588-2605, 2006.

[81] K. S. Bhatia, C. C. Cho, C. S. Tong, E. H. Yuen, and A. T. Ahuja, "Shear wave elasticity imaging of cervical lymph nodes," Ultrasound Med Biol, vol. 38, no. 2, pp. 195-201, 2012.

[82] B. Arnal, M. Pernot, and M. Tanter, "Monitoring of thermal ablation therapy based on shear modulus changes: Shear wave thermometry and shear wave lesion imaging," 2010 IEEE Int. Ultrason. Symp., vol. 58, no. 2, pp. 1522-1525, 2010.

[83] A. Mariani, W. Kwiecinski, M. Pernot, D. Balvay, M. Tanter, O. Clement, C. A. Cuenod, and F. Zinzindohoue, "Real time shear waves elastography monitoring of thermal ablation: In vivo evaluation in pig livers," J. Surg. Res., vol. 188, no. 1, pp. 37-43, 2014.

[84] E. Sapin, J. L. Gennisson, M. Pernot, M. Fink, and M. Tanter, "Temperature dependence of the shear modulus of soft tissues assessed by ultrasound," Proc. IEEE Ultrason. Symp., vol. 1701, 2009.

[85] L. Castera, J. Denis, G. Babany, and F. Roudot-Thoraval, "Evolving practices of non-invasive markers of liver fibrosis in patients with chronic hepatitis $\mathrm{C}$ in France: 
Time for new guidelines?," J. Hepatol., vol. 46, no. 3, pp. 528-529, 2007.

[86] M. Tanter and M. Fink, "Ultrafast imaging in biomedical ultrasound," IEEE Trans. Ultrason. Ferroelectr. Freq. Control, vol. 61, no. 1, pp. 102-119, 2014.

[87] M. L. Palmeri, M. H. Wang, N. C. Rouze, M. F. Abdelmalek, C. D. Guy, B. Moser, A. M. Diehl, and K. R. Nightingale, "Noninvasive evaluation of hepatic fibrosis using acoustic radiation force-based shear stiffness in patients with nonalcoholic fatty liver disease," J. Hepatol., vol. 55, no. 3, pp. 666-672, 2011.

[88] M. Golatta, M. Schweitzer-Martin, A. Harcos, S. Schott, C. Gomez, A. Stieber, G. Rauch, C. Domschke, J. Rom, F. Schutz, C. Sohn, and J. Heil, "Evaluation of virtual touch tissue imaging quantification, a new shear wave velocity imaging method, for breast lesion assessment by ultrasound," Biomed Res. Int., vol. 2014, p. ID 960262, 2014.

[89] E. M. Jung, A. G. Schreyer, D. Schacherer, C. Menzel, S. Farkas, M. Loss, S. Feuerbach, N. Zorger, and C. Fellner, "New real-time image fusion technique for characterization of tumor vascularisation and tumor perfusion of liver tumors with contrast-enhanced ultrasound, spiral CT or MRI: First results," Clin. Hemorheol. Microcirc., vol. 43, no. 1-2, pp. 57-69, 2009.

[90] V. Dutt, R. R. Kinnick, and J. F. Greenleaf, "Acoustic shear wave displacement measurement using ultrasound," 1996 IEEE Ultrason. Symp. Proc., vol. 2, pp. 1185-1188, 1996.

[91] N. Feng, J. Zhang, and W. Wang, "A quadrature demodulation method based on tracking the ultrasound echo frequency," Ultrasonics, vol. 44, no. SUPPL., pp. 4750, 2006.

[92] J. Silver, "Development of Ultrasound Based Techniques for Measuring Skeletal Muscle Motion," Carleton University, 2009.

[93] T. J. Hall, M. Bilgen, M. F. Insana, and T. a. Krouskop, "Phantom materials for elastography," IEEE Trans. Ultrason. Ferroelectr. Freq. Control, vol. 44, no. 6, pp. 1355-1365, 1997.

[94] R. E. Sheriff and B. S. Matisoff, "Encyclopedic Dictionary of Applied Geophysics," 
Book, pp. 351-393, 1982.

[95] M. Feldman, Hilbert Transform Applications in Mechanical Vibration. New York: Wiley, 2011.

[96] X. Fan and K. Hynynen, "The effect of wave reflection and refraction at soft tissue tissue interfaces during ultrasound hyperthermia treatments," J. Acoust. Soc. Am., vol. 91 , no. 3, pp. 1727-1736, 1992.

[97] K. R. Nightingale, M. S. Soo, R. W. Nightingale, and G. E. Trahey, "Acoustic radiation force impulse imaging: in vivo demonstration of clinical feasibility.," Ultrasound Med Biol, vol. 28, no. 2, pp. 227-235, 2002.

[98] M. L. Palmeri, M. H. Wang, J. J. Dahl, K. D. Frinkley, and K. R. Nightingale, "Quantifying Hepatic Shear Modulus In Vivo Using Acoustic Radiation Force," Ultrasound Med. Biol., vol. 34, no. 4, pp. 546-558, 2008.

[99] J. L. Gennisson, T. Deffieux, E. Macé, G. Montaldo, M. Fink, and M. Tanter, "Viscoelastic and anisotropic mechanical properties of in vivo muscle tissue assessed by supersonic shear imaging," Ultrasound Med. Biol., vol. 36, no. 5, pp. 789-801, 2010.

[100] J. G. Proakis, D. G. Monolakis, G. Proakis John, and G. Manolakis Dimitris, Digital signal processing: principles, algorithms, and applications, 3rd ed. New Jersy: Pentice Hall, 1996.

[101] K. Hoyt, T. Kneezel, B. Castaneda, and K. J. Parker, "Quantitative sonoelastography for the in vivo assessment of skeletal muscle viscoelasticity," Phys. Med. Biol., vol. 53, no. 15, pp. 4063-4080, 2008.

[102] R. Sinkus, J. Bercoff, M. Tanter, J. L. Gennisson, C. El Khoury, V. Servois, A. Tardivon, and M. Fink, "Nonlinear viscoelastic properties of tissue assessed by ultrasound," Ieee Trans. Ultrason. Ferroelectr. Freq. Control, vol. 53, no. 11, pp. 2009-2018, 2006.

[103] H. Zhao, P. Song, M. W. Urban, J. F. Greenleaf, and S. Chen, "Shear wave speed measurement using an unfocused ultrasound beam.," Ultrasound Med. Biol., vol. 38 , no. 9, pp. 1646-55, 2012. 
[104] J. R. Basford, T. R. Jenkyn, K. An, and R. L. Ehman, "Evaluation of Healthy and Diseased Muscle With Magnetic Resonance Elastography," vol. 83, no. November, pp. 1530-1536, 2002.

[105] Y. Zhu, C. Dong, Y. Yin, X. Chen, Y. Guo, Y. Zheng, Y. Shen, T. Wang, X. Zhang, and S. Chen, "The Role of Viscosity Estimation for Oil-in-gelatin Phantom in Shear Wave Based Ultrasound Elastography," Ultrasound Med. Biol., vol. 41, no. 2, pp. 601-609, 2015.

[106] L. Filipczyński, "Absorption of longitudinal and shear waves and generation of heat in soft tissues," Ultrasound Med. Biol., vol. 12, no. 3, pp. 223-228, Mar. 2017.

[107] M. O'Donnell and J. G. Miller, "Mechanisms of ultrasonic attenuation in soft tissue," Ultrason. Tissue Charact., vol. 2, pp. 37-40, 1979.

[108] R. Siegel and J. R. Howell, Thermal Radiation Heat Transfer. New York: Taylor \& Francis, 2002.

[109] Z. Qu and Y. Ono, "Ultrasonic Measurements of Shear Wave Propagation in Soft Tissue Phantom for Tissue Characterization," in Proc. IEEE International Symposium on Medical Measurements and Applications (MeMeA), 2013, pp. 4043.

[110] M. O. Culjat, D. Goldenberg, P. Tewari, and R. S. Singh, "A review of tissue substitutes for ultrasound imaging," Ultrasound Med. Biol., vol. 36, no. 6, pp. 861-873, 2010.

[111] K. Zell, J. I. Sperl, M. W. Vogel, R. Niessner, and C. Haisch, "Acoustical properties of selected tissue phantom materials for ultrasound imaging.," Phys. Med. Biol., vol. 52, no. 20, pp. N475-N484, 2007.

[112] M. M. Nguyen, S. Zhou, J. luc Robert, V. Shamdasani, and H. Xie, "Development of oil-in-gelatin phantoms for viscoelasticity measurement in ultrasound shear wave elastography," Ultrasound Med. Biol., vol. 40, no. 1, pp. 168-176, 2014.

[113] R. W. Hamming, Digital Filters (3rd Ed.). Hertfordshire, UK: Prentice Hall International (UK) Ltd., 1989. 
[114]J. Benesty, J. Chen, and Y. Huang, "Noncausal (frequency-domain) optimal filters," in Microphone array signal processing, Berlin, Germany: Springer, 2008, pp. 115-137.

[115] D. Falconer, S. L. Ariyavisitakul, A. Benyamin-Seeyar, and B. Eidson, "Frequency domain equalization for single-carrier broadband wireless systems," IEEE Communications Magazine, vol. 40, no. 4. pp. 58-66, 2002.

[116] J. Armstrong, "Peak-to-average power reduction for OFDM by repeated clipping and frequency domain filtering," Electronics Letters, vol. 38, no. 5. pp. 246-247, 2002.

[117] E. L. Madsen, M. A. Hobson, H. Shi, T. Varghese, and G. R. Frank, "Tissuemimicking agar/gelatin materials for use in heterogeneous elastography phantoms.," Phys. Med. Biol., vol. 50, no. 23, pp. 5597-618, 2005.

[118] C. Joly-Duhamel, D. Hellio, and M. Djabourov, "All Gelatin Networks:1. Biodiversity and Physical Chemistry," Langmuir, vol. 18, pp. 7208-7217, 2002.

[119] W. G. Mayer, "Energy partition of ultrasonic waves at flat boundaries," Ultrasonics, vol. 3, no. 2, pp. 62-68, 1965.

[120] B.A.Auld, Acoustic fields and waves in solids, volume II. Malabar: Krieger, 1975.

[121] S.-R. Huang, "Time domain Doppler estimators of the amplitude of vibrating targets," J. Acoust. Soc. Am., vol. 91, no. 2, p. 965, 1992.

[122] S.-R. Huang, R. M. Lerner, and K. J. Parker, "On estimating the amplitude of harmonic vibration from the Doppler spectrum of reflected signals," J. Acoust. Soc. Am., vol. 88, no. 6, pp. 2702-2712, 1990. 